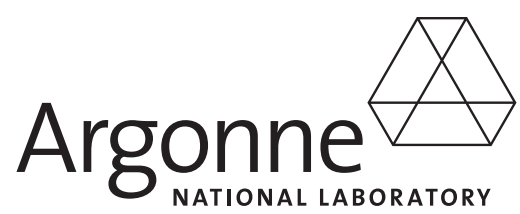

ANL/EVS/AGEM/TR-09-03

\title{
October 2008 Monitoring Results for Morrill, Kansas
}

\section{Environmental Science Division}


About Argonne National Laboratory

Argonne is a U.S. Department of Energy laboratory managed by UChicago Argonne, LLC under contract DE-AC02-06CH11357. The Laboratory's main facility is outside Chicago, at 9700 South Cass Avenue, Argonne, Illinois 60439. For information about Argonne and its pioneering science and technology programs, see www.anl.gov.

\section{Availability of This Report}

This report is available, at no cost, at http://www.osti.gov/bridge. It is also available on paper to the U.S. Department of Energy and its contractors, for a processing fee, from:

U.S. Department of Energy

Office of Scientific and Technical Information

P.O. Box 62

Oak Ridge, TN 37831-0062

phone (865) 576-8401

fax (865) 576-5728

reports@adonis.osti.gov

\section{Disclaimer}

This report was prepared as an account of work sponsored by an agency of the United States Government. Reference herein to any specific commercial product, process, or service by trade name, trademark, manufacturer, or otherwise, does not necessarily constitute or imply its endorsement, recommendation, or favoring by the United States Government or any agency thereof. The views and opinions of document authors expressed herein do not necessarily state or reflect those of the United States Government or any agency thereof, Argonne National Laboratory, or UChicago Argonne, LLC. 


\section{October 2008 Monitoring Results for Morrill, Kansas}

by

Applied Geosciences and Environmental Management Section

Environmental Science Division, Argonne National Laboratory

March 2009

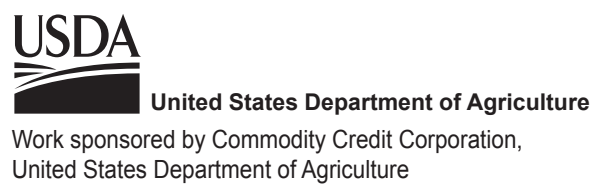




\section{Contents}

Notation.

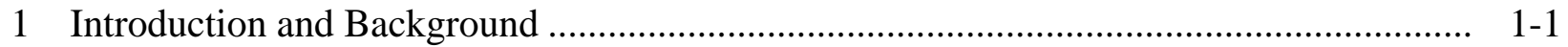

2 Sample Collection and Analysis Activities......................................................... 2-1

2.1 Measurement of Groundwater Levels.............................................................. 2-1

2.2 Monitoring and Private Well Sampling and Analyses............................................. 2-1

2.3 Surface Water and Sediment Sampling and Analyses........................................... 2-2

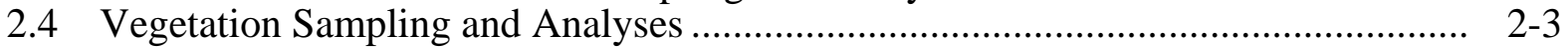

2.5 Handling and Disposal of Investigation-Derived Waste ....................................... 2-4

2.6 Quality Control for Sample Collection, Handling, and Analysis ........................... 2-4

3 Results and Discussion ......................................................................................

3.1 Groundwater Level Data.................................................................................. 3-1

3.2 Groundwater Analysis Results............................................................................ $3-2$

3.3 Surface Water and Sediment Analysis Results .................................................... 3-3

3.4 Vegetation Analysis Results and Observations ................................................ 3-4

3.5 Comparison of Analytical Results for Samples Collected after Low-Flow Purging or Purging of Three Well Volumes .................................................... 3-5

4 Conclusions and Recommendations ................................................................. $4-1$

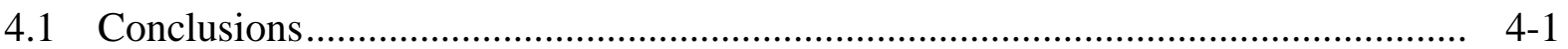

4.2 Issues and Ongoing Work ........................................................................... $4-2$

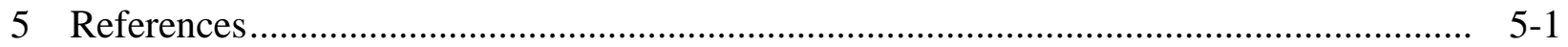

Appendix A: Sequence of Sampling Activities at Morrill, Kansas, in October 2008 ........... A-1

Appendix B: Analytical Results for Waste Purge Water .............................................. B-1

Appendix C: $\quad$ Data Summary for Verification VOCs Analyses by TestAmerica .................. C-1

\section{Figures}

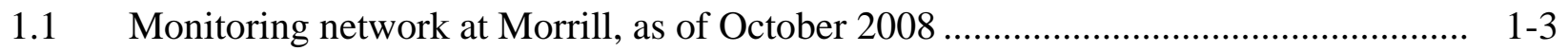

2.1 Locations of surface water and creek bed sediment sampling along Terrapin Creek at Morrill in October 2008 ............................................................... 2-7

2.2 Locations of native vegetation sampling downgradient of the former CCC/USDA facility and along Terrapin Creek at Morrill in July 2008 ..................... 2-8

3.1a Potentiometric surface at Morrill, based on water levels measured manually on January 8, 2007 


\section{Figures (cont.)}

3.1b Potentiometric surface at Morrill, based on water levels measured manually on July 27, 2007

3.1c Potentiometric surface at Morrill, based on water levels measured manually on April 30, 2008

3.1d Potentiometric surface at Morrill, based on water levels measured manually on September 19, 2008

3.2a Hydrographs summarizing results of long-term water level monitoring in wells MW1S-MW4S and MW6S-MW8S at Morrill, from January 1, 2007, to

December 31, 2007

3.2b Hydrographs summarizing results of long-term water level monitoring in wells MW1S-MW4S and MW6S-MW8S at Morrill, from January 1, 2008, to

September 19, 2008

3.3 Carbon tetrachloride concentrations in groundwater at Morrill, October 2008.

3.4 Lateral extent of the carbon tetrachloride contamination in groundwater at Morrill, as interpreted on the basis of sampling and analysis in October 2007 and April 2008

3.5 Lateral extent of the carbon tetrachloride contamination in groundwater at Morrill, as interpreted on the basis of sampling and analysis in October 2008

\section{Tables}

3.1 Hand-measured groundwater levels at Morrill in 2007 and 2008

3.2 Results of analyses at the AGEM Laboratory for volatile organic compounds in groundwater samples collected at Morrill, October 2003 to October 2008.

3.3 Field measurements for groundwater samples collected at Morrill, October 2003 to October 2008

3.4 Results of analyses at the AGEM Laboratory for volatile organic compounds in surface water and sediment samples collected at Morrill, March 2007 to October 2008. 


\section{Tables (cont.)}

3.5 Results of analyses at the AGEM Laboratory for carbon tetrachloride and chloroform in vegetation samples collected at Morrill, October 2006 to

July 2008

3.6 Results from the AGEM Laboratory and TestAmerica for sampling of selected wells with low-flow purging method or purging of three well volumes

A.1 Sequence of sampling activities in July 2008 and October 2008 at Morrill, Kansas..... 


\section{Notation}

AGEM Applied Geosciences and Environmental Management

AMSL above mean sea level

BGL below ground level

${ }^{\circ} \mathrm{C}$ degree(s) Celsius

CCC Commodity Credit Corporation

DO dissolved oxygen

EPA U.S. Environmental Protection Agency

$\mathrm{ft} \quad$ foot (feet)

gal gallon(s)

in. inch(es)

KDHE Kansas Department of Health and Environment

L liter(s)

$\mu \mathrm{g} / \mathrm{kg} \quad$ microgram(s) per kilogram

$\mu \mathrm{g} / \mathrm{L} \quad$ microgram(s) per liter

$\mu \mathrm{S} / \mathrm{cm} \quad$ microsiemen(s) per centimeter

$\mathrm{mg} / \mathrm{L} \quad$ milligram(s) per liter

min minute(s)

$\mathrm{mV}$ millivolt(s)

ORP oxidation-reduction potential

RPD relative percent difference

SOP standard operating procedure

TOC top of casing

USDA U.S. Department of Agriculture

VOC volatile organic compound 


\section{October 2008 Monitoring Results for Morrill, Kansas}

\section{Introduction and Background}

In September 2005, the Commodity Credit Corporation of the U.S. Department of Agriculture (CCC/USDA) initiated periodic sampling of groundwater in the vicinity of a grain storage facility formerly operated by the CCC/USDA at Morrill, Kansas. The sampling at Morrill is being performed on behalf of the CCC/USDA by Argonne National Laboratory, in accord with a monitoring program approved by the Kansas Department of Health and Environment (KDHE 2005), to monitor levels of carbon tetrachloride contamination identified in the groundwater at this site (Argonne 2004, 2005a). This report provides results for the most recent monitoring event, in October 2008.

Under the KDHE-approved monitoring plan (Argonne 2005b), groundwater was initially sampled twice yearly for a period of two years (in fall 2005, in spring and fall 2006, and in spring and fall 2007). The samples were analyzed for volatile organic compounds (VOCs), as well as for selected geochemical parameters to aid in the evaluation of possible natural contaminant degradation (reductive dechlorination) processes in the subsurface environment. During the two-year period, the originally approved scope of the monitoring was expanded to include vegetation sampling (initiated in October 2006) and surface water and stream bed sediment sampling (initiated in March 2007, after a visual reconnaissance along Terrapin Creek [Argonne 2007a]).

The analytical results for groundwater sampling events at Morrill in September 2005, March and September 2006, March and October 2007, and April 2008 were documented previously (Argonne 2006a,b, 2007b, 2008a,c). Those results consistently demonstrated the presence of carbon tetrachloride contamination, at levels exceeding the KDHE Tier 2 risk-based screening level $(5.0 \mu \mathrm{g} / \mathrm{L})$ for this compound, in a groundwater plume extending generally southsoutheastward from the former CCC/USDA facility, toward Terrapin Creek at the south edge of the town. Low levels $(\leq 1.3 \mu \mathrm{g} / \mathrm{L})$ of carbon tetrachloride were persistently detected at monitoring well MW8S on the bank of an intermittent tributary to Terrapin Creek. This observation suggested a possible risk of contamination of the surface waters of the creek. That concern became the driving force for ongoing monitoring and consideration of possible remedial options for Morrill. 
In light of the early findings, in 2006 the CCC/USDA recommended expansion of the approved monitoring program to include the collection and analysis of surface water samples along Terrapin Creek (Argonne 2006a). At the request of the KDHE (2007a), locations for both surface water and shallow sediment sampling were discussed with the KDHE in January 2007. An addendum to the existing monitoring plan and a standard operating procedure (SOP AGEM-15) for sediment sampling were submitted to the KDHE on the basis of these discussions (Argonne 2007c,d). To supplement the original scope of the monitoring, Argonne also sampled natural vegetation along Terrapin Creek in October 2006, April 2007, and July 2007 for analyses for VOCs. The results of these plant tissue analyses were reported previously (Argonne 2008a).

The October 2008 sampling event reported here represents a continuation of the two-year monitoring program, as requested by the KDHE (2007b). The groundwater sampling is presently conducted, in accord with the monitoring plan (Argonne 2005b) and the addendum to that plan (Argonne 2007a), in a network of 12 monitoring wells and 3 private wells (Figure 1.1), at locations approved by the KDHE. 


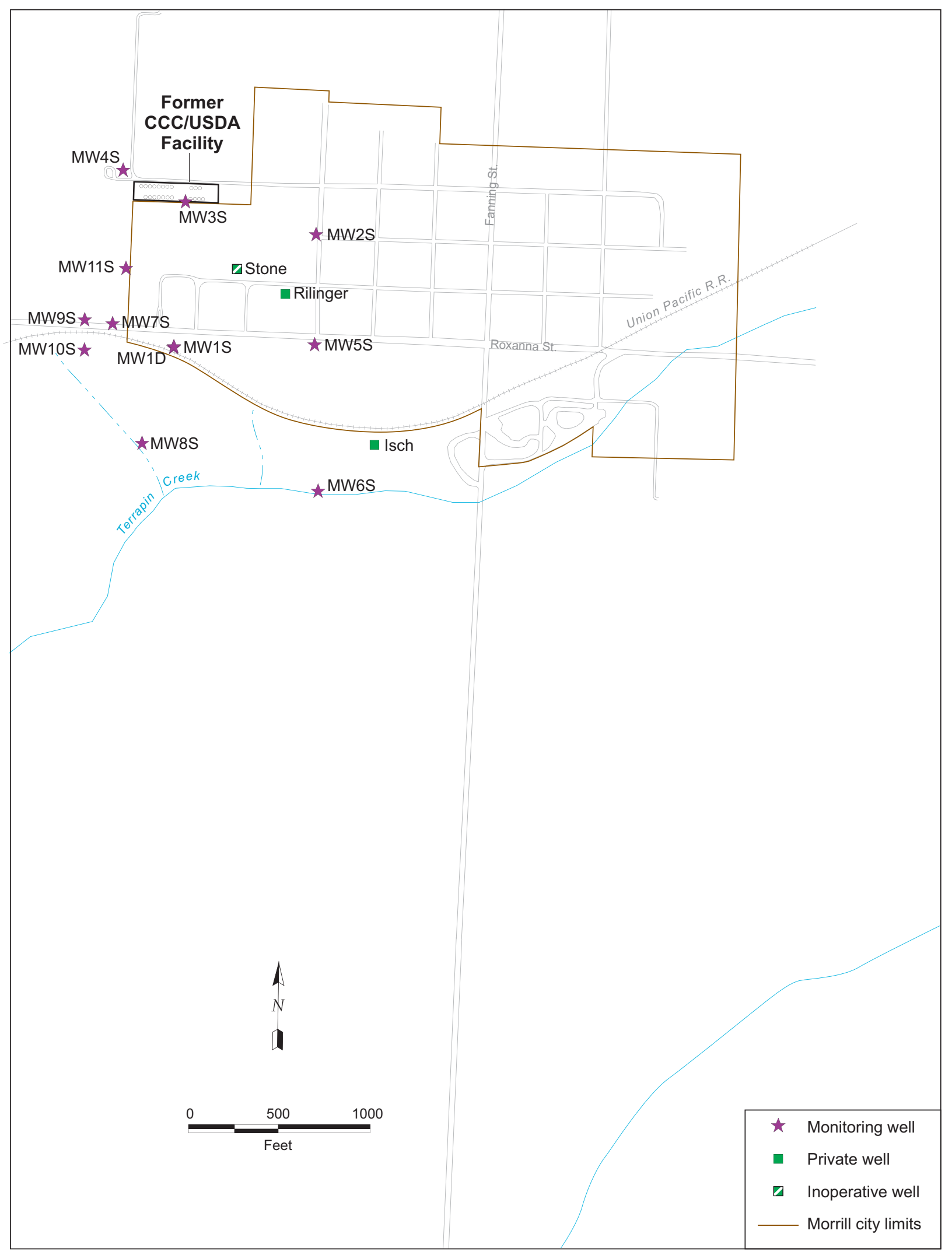

FIGURE 1.1 Monitoring network at Morrill, as of October 2008. 


\section{Sample Collection and Analysis Activities}

\subsection{Measurement of Groundwater Levels}

Before each well was purged in preparation for sampling on October 20-21, 2008, a water level indicator was used (where possible) to measure the depth to groundwater and the total depth from the top of the well casing, with an accuracy of $\pm 0.01 \mathrm{ft}$.

Data recorders currently installed in wells MW1S-MW4S and MW6S-MW8S are gathering long-term data on the groundwater elevation and gradient at Morrill. The data recorders in these wells were downloaded on January 8 and July 27, 2007; on April 30, 2008; and most recently on September 19, 2008. Water levels were measured manually in all monitoring wells on these dates, as well as in conjunction with the sampling events on October 1-3, 2007; April 14-15, 2008; and October 20-21, 2008.

The groundwater level data are discussed in Section 3.1.

\subsection{Monitoring and Private Well Sampling and Analyses}

Monitoring wells MW1D and MW1S-MW11S and the Stone, Isch, and Rilinger private wells (Figure 1.1) were sampled on October 20-21, 2008.

Samples were collected from monitoring wells by using a low-flow bladder pump. After measurement of water levels, each monitoring point was purged of a small volume in accord with U.S. Environmental Protection Agency (EPA) procedure EPA/540/S-95/504 (Puls and Barcelona 1996; Yeskis and Zavala 2002) and the equipment manufacturers' instructions. Field measurements of temperature, $\mathrm{pH}$, conductivity, dissolved oxygen (DO), and oxidation reduction potential (ORP) were taken during purging until the measurements stabilized. Field measurements of iron(II) were made as outlined in the monitoring plan (Argonne 2005b), in accord with procedures in the Master Work Plan (Argonne 2002). Samples from the Isch and Rilinger private wells were collected after a 5-min purge with the dedicated pump. The sample from the Stone private well was collected after purging of the well by bailing. 
At the request of KDHE (2008), samples were also collected from selected monitoring wells (MW1S, MW2S, MW3S, and MW11S) after purging of three well volumes to verify that samples collected with the low-flow method were representative of site conditions. This comparison sampling is discussed further in Section 3.5.

The sequence of activities during the October 2008 sampling event is summarized in Appendix A.

Groundwater samples for VOCs analyses were collected in appropriate laboratory containers, labeled, packaged, and chilled to $4^{\circ} \mathrm{C}$ by placement in ice-filled coolers. The samples were shipped via an overnight delivery service to the Applied Geosciences and Environmental Management (AGEM) Laboratory at Argonne for VOCs analyses with EPA Method 524.2 (EPA 1995). Separate aliquots of selected samples (chosen in the field) were shipped to TestAmerica Laboratories, Inc., South Burlington, Vermont, for verification VOCs analysis.

As recommended at the end of the original two-year monitoring period (Argonne 2008a), routine analyses for the attenuation parameters sulfate, sulfide, methane, total organic carbon, carbon dioxide, alkalinity, chloride, and dissolved hydrogen were discontinued after the October 2007 sampling event.

The groundwater analysis results are presented and discussed in Section 3.2.

\subsection{Surface Water and Sediment Sampling and Analyses}

At the request of the KDHE (2007a), surface water samples and corresponding samples of the underlying shallow sediments in the creek bed were collected for VOCs analyses in March and October 2007 and in April and October 2008 at five locations along Terrapin Creek (Figure 2.1), as outlined in the addendum (Argonne 2007c) to the monitoring plan (Argonne 2005b). The sampling was conducted in accord with procedures in the Master Work Plan (Argonne 2002) and SOP AGEM-15 (Argonne 2007d). Surface water flow in Terrapin Creek south of Morrill originates at the outfall from an earthen dam and retention pond approximately 1,900 ft southwest of the former CCC/USDA facility (Figure 2.1). Surface water and sediment sampling location SMB, which is directly downstream of this outfall, is believed to lie upgradient, or cross-gradient, to groundwater flow (and hence possible contaminant migration) 
from the vicinity of the former CCC/USDA facility. (See Section 3.1.) Sampling locations SM1SM4 were selected to lie downgradient and downstream from the carbon tetrachloride detections previously identified at MW8S and elsewhere in the monitoring well network.

Samples of surface water were collected in appropriate containers, labeled, preserved at $4{ }^{\circ} \mathrm{C}$, and shipped by an overnight delivery service to the AGEM Laboratory for VOCs analyses with EPA Method 524.2 (EPA 1995). Samples of the shallow creek bed sediments were collected by scooping the materials directly into appropriate laboratory containers (Argonne 2007d). The samples were labeled, preserved on dry ice, and shipped to the AGEM Laboratory for sample preparation and VOCs analyses with modified EPA Methods 5030B and 8260B.

The surface water and sediment analysis results are presented and discussed in Section 3.3.

\subsection{Vegetation Sampling and Analyses}

Samples of natural vegetation, consisting of branch and leaf tissue material recovered from mature trees, were previously collected on July 26, 2007, at 18 locations (points 001-018 in Figure 2.2) along Terrapin Creek and its tributaries south and southeast of the former CCC/USDA facility. The sampling locations were selected to lie in the apparent direction of groundwater flow from the former facility; they formed a baseline study for evaluation of potential phytoremediation.

The monitoring event in April 2008 did not include vegetation sampling for the detection of carbon tetrachloride and chloroform. The experience gained by the CCC/USDA and Argonne in monitoring phytoremediation treatment programs in Nebraska and Illinois indicates that little uptake of contaminants into tree tissues is expected early in the growing season (e.g., Argonne 2008b).

Tree sampling conducted on July 24, 2008, included an expansion of the baseline sampling locations (points 001-024 in Figure 2.2). The tissue samples were collected in appropriate laboratory containers, labeled, preserved on dry ice, and shipped to the AGEM Laboratory for carbon tetrachloride and chloroform analyses by a headspace technique based on 
a modification of EPA Method 5021 (http://www.epa.gov/epahome/index/; Alvarado and Rose 2004).

The analytical results for the vegetation samples are presented and discussed in Section 3.4.

\subsection{Handling and Disposal of Investigation-Derived Waste}

The small volume of purge water generated as potentially contaminated investigationderived waste was containerized on-site. The accumulated purge water was sampled and analyzed for VOCs (including ethylene dibromide) and nitrates by a KDHE-certified laboratory. The analytical results (Appendix B) indicated no detectable concentrations of carbon tetrachloride, chloroform, ethylene dibromide, or nitrate. The water was discharged on-site on December 11, 2008, with the verbal approval of the KDHE.

\subsection{Quality Control for Sample Collection, Handling, and Analysis}

The quality control/quality assurance procedures followed during the October 2008 monitoring event are described in detail in the Master Work Plan (Argonne 2002) and SOP AGEM-15 (Argonne 2007b). These procedures are summarized as follows:

- Sample collection and handling activities were monitored by the documentation of samples as they were collected and the use of chain-ofcustody forms and custody seals to ensure sample integrity during handling and shipment.

- Samples designated for VOCs analyses were received with custody seals intact and at the appropriate preservation temperature. All samples were analyzed within the required holding times.

- Quality control samples (equipment rinsates and trip blanks) collected to monitor sample collection and handling activities, were free of carbon tetrachloride contamination. Trace levels of chloroform $(<1 \mu \mathrm{g} / \mathrm{L})$ present in the samples are consistent with levels found previously in the source water. 
Method blanks used to monitor analytical methodologies were free of carbon tetrachloride and chloroform contamination.

- Groundwater, surface water, and sediment samples were analyzed for VOCs at the AGEM Laboratory with the purge-and-trap method on a gas chromatograph-mass spectrometer system. Calibration checks with each sample delivery group were required to be within $\pm 20 \%$ of the standard. Surrogate standard determinations performed on samples and blanks were within the specified range of $80-120 \%$ for all samples, in either the initial analysis or a successful reanalysis. The low relative percent difference (RPD) values for duplicate analyses of selected samples $(<2 \%)$ demonstrated consistency in the analytical methodology.

- In accordance with the procedures defined in the Master Work Plan (Argonne 2002), the analyses of water samples at the AGEM Laboratory were verified by a second laboratory. Nine groundwater samples collected during the October 21-22, 2008, monitoring event were also submitted to TestAmerica for analysis according to the EPA's Contract Laboratory Program methodology. Results show good agreement over the range of contaminant concentrations detected, with average RPD values of $13.6 \%$ for carbon tetrachloride and 3.2\% for chloroform. Summary pages for the verification organic analyses by TestAmerica for sampling conducted on October 20-21, 2008, are in Appendix C.

- Vegetation samples were analyzed for carbon tetrachloride and chloroform at the AGEM Laboratory by using a gas chromatograph with electron capture detection to achieve the low detection limits required. An 11-point calibration of the gas chromatograph system was established on the basis of the mass of known quantities of carbon tetrachloride and chloroform.

- To verify the suitability of the low-flow sampling methodology for obtaining representative samples from the monitoring wells at Morrill, at the request of the KDHE (2008), four of the wells were also sampled after purging of three well volumes. Results suggest that the low-flow technique is acceptable but that the positioning of the pump intake relative to the well screen and static 
water level at the time of sampling is critical in obtaining a representative sample with this method. The results of the comparison sampling are discussed further in Section 3.5. 


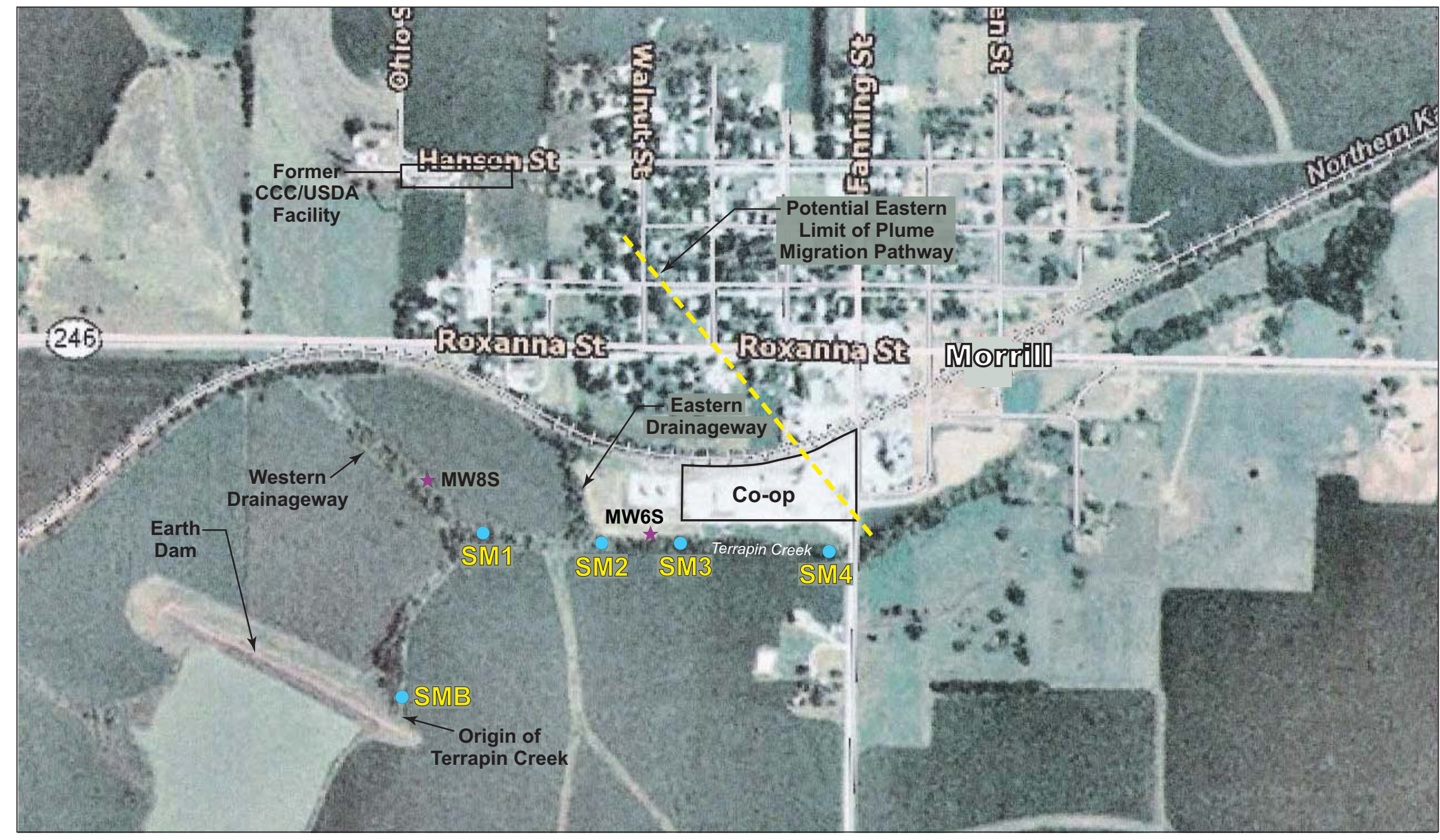

FIGURE 2.1 Locations of surface water and creek bed sediment sampling (SM1-SM4, SMB) along Terrapin Creek at Morrill in October 2008. 


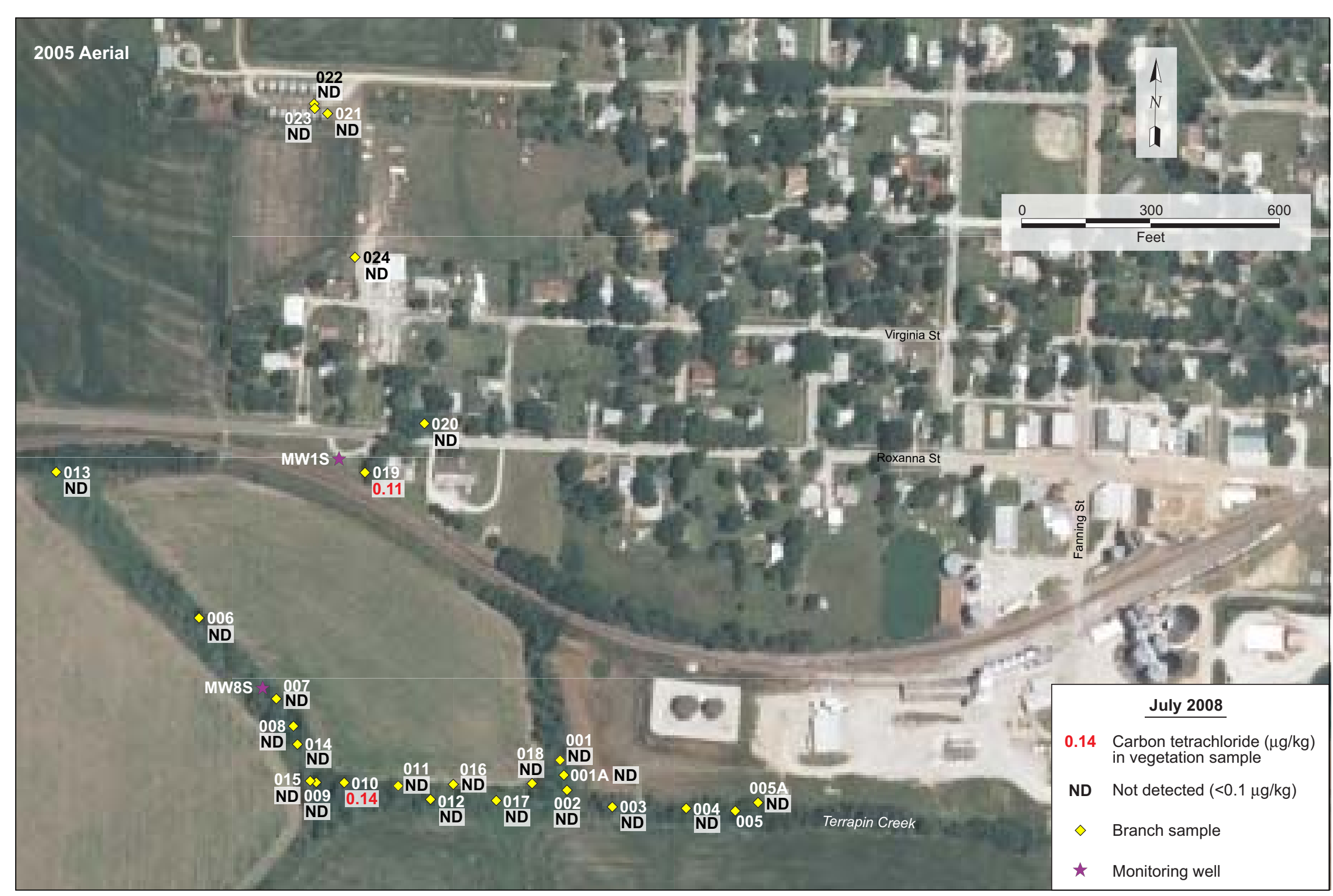

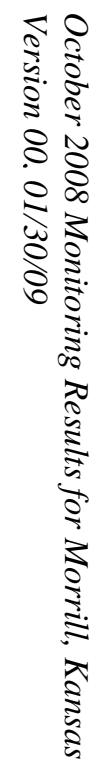

FIGURE 2.2 Locations of native vegetation sampling (001-024) downgradient of the former CCC/USDA facility and along Terrapin Creek at Morrill in July 2008. 


\section{Results and Discussion}

\subsection{Groundwater Level Data}

Depths to groundwater were measured manually in all available monitoring wells on January 8 and July 27, 2007; October 1-3, 2007 (during sampling); April 14-15, 2008 (during sampling); April 30 and September 19, 2008; and October 20-21, 2008 (during sampling). The hand-measured water level data are in Table 3.1.

Earlier estimates of the potentiometric surface at Morrill, based on manual measurements on January 8 and July 27, 2007, are depicted in Figures 3.1a and 3.1b, respectively. These results indicate a groundwater flow direction toward the south-southeast from the former CCC/USDA facility. Persistently low water levels observed at MW11S empirically suggest the apparent presence of a groundwater "sink" southwest of the former facility in the vicinity of this monitoring well. This observation is likely an artifact, however, associated with the greater depth of completion of well MW11S in the aquifer unit and the local groundwater flow system (both horizontal and vertical) at this location, relative to nearby wells MW3S, MW4S, and MW7S.

The potentiometric surface at Morrill, as interpreted from manual measurements on April 30, 2008, is depicted in Figure 3.1c. This diagram depicts a slight shift in groundwater flow directions toward the west, relative to the previous determinations (January 8 and July 27, 2007; Figures 3.1a and 3.1b). Data from the long-term recording transducers installed in monitoring wells MW1S-MW4S and MW6S-MW8S, summarized in Figures 3.2a,b, suggest a possible mechanism for this occurrence.

The hydrographs in Figures 3.2a,b indicate that transient fluctuations in groundwater levels have occurred in response to both seasonal and shorter-term variations in rainfall during the period of observation (January 2007 through September 2008); however, a generally increasing trend from January 2007 to June 2008 is apparent. This trend reached a relative peak (to date) during the spring and early summer of 2008. Under these high-groundwater conditions, an increase in the upstream occurrence of surface water flow in the tributary drainage southwest of monitoring wells MW9S and MW10S (along which drainage well MW8S lies; Figure 3.1c) was visually observed during the April 30, 2008, monitoring. The data in Figure 3.1c and Table 3.1 suggest that enhanced groundwater seepage might have contributed to the surface 
drainage observed along the western tributary on April 30, 2008, temporarily influencing the pattern of groundwater flow in the aquifer in the vicinity of Terrapin Creek.

Figure 3.2b indicates that groundwater levels generally receded (by as much as $10 \mathrm{ft}$ ) at Morrill during the later summer and fall of 2008. The potentiometric surface at Morrill, based on manual measurements on September 19, 2008 (Figure 3.1d), indicates a return to the more southsoutheasterly flow direction evident under the (lower) groundwater level conditions observed in 2007.

The hydrograph in Figure 3.2b for monitoring well MW6S is marked by a series of sharp downward "spikes" in the water level at this location during July and August of 2008. The observed events are believed to reflect transient drawdown in response to pumping of the Grimm irrigation well, which was installed just south of the MW6S location in March 2008 (Argonne 2008c). Little or no distinct response to the pumping of this well is apparent at the other monitored well locations; however, the operation of the Grimm well empirically coincides with the decline in water levels observed at all locations in summer and fall 2008. A similar seasonal decline in water levels was also observed in summer and fall 2007, in the absence of the Grimm well pumping; additional monitoring will therefore be required for further determination of the potential areal and longer-term hydraulic influence of this well on groundwater levels and migration patterns in the Morrill study area.

\subsection{Groundwater Analysis Results}

The analytical data for VOCs in the groundwater samples collected in October 2008 are in Table 3.2, together with data for the previous sampling events conducted under the KDHEapproved monitoring plan (Argonne 2005b). The October 2008 data for carbon tetrachloride in groundwater are illustrated in Figure 3.3.

Carbon tetrachloride was detected at 9 of the 15 monitoring locations, at concentrations ranging from $<1 \mu \mathrm{g} / \mathrm{L}$ (in the Rilinger private well) to a maximum of $63 \mu \mathrm{g} / \mathrm{L}$ at MW3S. Low levels of chloroform ( $<1 \mu \mathrm{g} / \mathrm{L}$ to a maximum of $1.8 \mu \mathrm{g} / \mathrm{L}$ at $\mathrm{MW} 1 \mathrm{~S})$ were detected in association with carbon tetrachloride at three of the monitoring locations (Table 3.2). 
Relatively consistent contaminant concentrations at each sampling location were observed in monitoring events in 2003-2007 (Table 3.2) and October 2008. In contrast, the April 2008 results indicate significant, apparently transient decreases in carbon tetrachloride at the following three locations:

- MW3S, at the southern boundary of the former CCC/USDA facility

- MW1S, downgradient from the former CCC/USDA facility and along the centerline of the plume

- MW5S, east of MW1S and near the eastern boundary of the plume

As noted in Section 3.1, the April 2008 sampling event took place during a period of very high groundwater levels (as a result of an extended trend of groundwater recharge to the Morrill aquifer), immediately after several heavy rainfalls (Figure 3.1c; Figures 3.2a,b; Table 3.2). Figure 3.4 compares the plume configuration in October 2007 with the configuration in April 2008. In October 2008, both the groundwater elevations across the site and the observed carbon tetrachloride concentrations at MW1S, MW3S, and MW5S returned to levels similar to those historically present in 2003-2007. Figure 3.5 indicates the position of the contaminant plume in October 2008.

Chloroform concentrations detected during the October 2008 sampling, like the carbon tetrachloride concentrations, were consistent with historical results before the April 2008 sampling (when unusually high water levels and rainfall were observed; Table 3.2).

The results of field measurements on the groundwater samples are in Table 3.3.

\subsection{Surface Water and Sediment Analysis Results}

Table 3.4 presents the results of VOCs analyses of the surface water and shallow sediment samples collected (at the request of the KDHE) along Terrapin Creek.

No carbon tetrachloride was detected in the surface water samples at an analytical method detection limit of $0.1 \mu \mathrm{g} / \mathrm{L}$. Similarly, no carbon tetrachloride was identified in the 
associated sediment samples at an analytical method detection limit of $1.0 \mu \mathrm{g} / \mathrm{kg}$. The October 2008 results therefore indicate, as did results for prior sampling events, that the surface waters and underlying sediments of Terrapin Creek have not been affected by carbon tetrachloride contamination.

\subsection{Vegetation Analysis Results and Observations}

The results of sampling of natural vegetation on July 26, 2007, at 18 locations along Terrapin Creek and its intermittent tributaries south and southeast of the former CCC/USDA facility are in Table 3.5. These results (and the results of earlier vegetation sampling) were discussed previously (Argonne 2008a).

During the winter of 2007-2008, severe ice storms in the Morrill area decimated trees in the town and along the creek. Many trees designated for sampling as part of the continuing vegetation monitoring were severely affected, with losses of large branches and treetops. As the 2008 growing season progressed, most of these trees recovered from the damage by producing new growth and good foliage, although with reduced crowns. Sampling on July 24, 2008, confirmed the earlier baseline sampling results, except at one tree location (005A; Figure 2.2). At this location, the maple sampled in the past was replaced in the sampling set by a willow growing immediately to the west. In addition, six new sampling points were established (019-024; Figure 2.2) along Roxanna Road and around the former CCC/USDA facility. Both herbaceous and woody plants were sampled at the new locations.

The analytical data for carbon tetrachloride and chloroform in the branch samples collected in July 2008 (and in previous years) are in Table 3.5. Trace concentrations of carbon tetrachloride were identified in July 2008 at two of the vegetation sampling locations (010 and 019; Figure 2.2), at an analytical method detection limit of $0.1 \mu \mathrm{g} / \mathrm{kg}$. Sampling point 019 lies near the previously identified central axis of the groundwater plume (Figures 2.2, 3.4, and 3.5); the July 2008 detection of carbon tetrachloride in the vegetation sample at this location is therefore qualitatively supported by the identified presence of carbon tetrachloride in nearby monitoring well MW1S (Figures 2.2 and 3.5). The detection at location 010 in July 2008 is not corroborated, however, by data obtained at any adjacent vegetation sampling point along Terrapin Creek during this sampling event or the July 2007 sampling; this detection in July 2008 will require continued monitoring for possible confirmation. 
With the possible exception of sampling point 010 noted above, the observed results for sampling locations 001-018 (along and rear Terrapin Creek; Figure 2.2) are qualitatively consistent with the absence of carbon tetrachloride in surface waters and shallow sediments discussed in Section 3.3, as well as with the non-detectable to trace concentrations of carbon tetrachloride identified in the shallow groundwater at monitoring well location MW8S. Experience in the CCC/USDA's project at Murdock, Nebraska (Argonne 2008b), indicates that the mature trees along the creek banks would readily take up carbon tetrachloride if it was present in water within reach of their root systems; the contaminant would then be observed in the tissue analyses.

\subsection{Comparison of Analytical Results for Samples Collected after Low-Flow Purging or Purging of Three Well Volumes}

At the request of the KDHE (2008), selected wells were sampled by using both the lowflow purging technique and the three-well-volume purging technique in order to confirm the suitability of the low-flow sampling method at the Morrill site. Samples collected from wells MW1S, MW2S, MW3S, and MW11S by using both methods were analyzed at the AGEM Laboratory and by TestAmerica. A comparison of the results is in Table 3.6.

Except for MW1S, the contaminant concentrations obtained with the two sampling methods compare favorably. At MW1S, the low-flow sampling approach was implemented incorrectly in the October 2008 sampling. The pump intake was positioned too near the static water level in the well, because of a decline in the groundwater level relative to the April 2008 sampling event. For the remaining three wells, the differences between concentrations reported by the AGEM Laboratory for comparison samples collected with the two methods are similar to the differences observed between the AGEM Laboratory results and the TestAmerica results for corresponding verification samples (Table 3.6).

In view of the incorrect positioning of the pump intake, the low contaminant concentrations in the sample collected from well MW1S with the low-flow method is considered non-representative of site conditions. In this instance, the full-purge value is honored instead. 
TABLE 3.1 Hand-measured groundwater levels at Morrill in 2007 and 2008.

\begin{tabular}{|c|c|c|c|c|c|c|c|c|c|c|c|}
\hline \multirow[b]{2}{*}{ Well } & \multirow[b]{2}{*}{$\begin{array}{l}\text { Top of Casing } \\
\text { Elevation } \\
\text { (ft AMSL) }\end{array}$} & \multicolumn{2}{|c|}{ January 8,2007} & \multicolumn{2}{|c|}{ July 27,2007} & \multicolumn{2}{|c|}{ October 1-3, 2007} & \multicolumn{2}{|c|}{ April 14-15, 2008} & \multicolumn{2}{|c|}{ April 30, 2008} \\
\hline & & $\begin{array}{l}\text { Depth to } \\
\text { Water } \\
\text { (ft BGL) }\end{array}$ & $\begin{array}{l}\text { Groundwater } \\
\text { Elevation } \\
\text { (ft AMSL) }\end{array}$ & $\begin{array}{l}\text { Depth to } \\
\text { Water } \\
\text { (ft BGL) }\end{array}$ & $\begin{array}{l}\text { Groundwater } \\
\text { Elevation } \\
\text { (ft AMSL) }\end{array}$ & $\begin{array}{l}\text { Depth to } \\
\text { Water } \\
\text { (ft BGL) }\end{array}$ & $\begin{array}{l}\text { Groundwater } \\
\text { Elevation } \\
\text { (ft AMSL) }\end{array}$ & $\begin{array}{l}\text { Depth to } \\
\text { Water } \\
\text { (ft BGL) }\end{array}$ & $\begin{array}{l}\text { Groundwater } \\
\text { Elevation } \\
\text { (ft AMSL) }\end{array}$ & $\begin{array}{l}\text { Depth to } \\
\text { Water } \\
\text { (ft BGL) }\end{array}$ & $\begin{array}{l}\text { Groundwater } \\
\text { Elevation } \\
\text { (ft AMSL) }\end{array}$ \\
\hline MW1S & 1124.68 & 26.79 & 1097.89 & 22.20 & 1102.48 & 21.65 & 1103.03 & 16.20 & 1108.48 & 15.96 & 1108.72 \\
\hline MW1D & 1124.63 & 26.43 & 1098.20 & 22.17 & 1102.46 & 22.75 & 1101.88 & 29.51 & 1095.12 & - & - \\
\hline MW2S & 1137.07 & 37.83 & 1099.24 & 30.50 & 1106.57 & 31.17 & 1105.90 & 23.55 & 1113.52 & 22.84 & 1114.23 \\
\hline MW3S & 1135.76 & 31.73 & 1104.03 & 22.31 & 1113.45 & 22.70 & 1113.06 & 16.95 & 1118.81 & 15.34 & 1120.42 \\
\hline MW4S & 1143.61 & 42.72 & 1100.89 & 30.18 & 1113.43 & 31.11 & 1112.50 & 26.32 & 1117.29 & 24.71 & 1118.90 \\
\hline MW5S & 1122.21 & 26.50 & 1095.71 & 18.47 & 1103.74 & 19.55 & 1102.66 & 11.20 & 1111.01 & 10.75 & 1111.46 \\
\hline MW6S & 1090.97 & 4.93 & 1086.04 & 4.77 & 1086.20 & 5.00 & 1085.97 & 5.15 & 1085.82 & 4.89 & 1086.08 \\
\hline MW7S & 1119.86 & 20.40 & 1099.46 & 13.28 & 1106.58 & 12.40 & 1107.46 & 7.72 & 1112.14 & 7.32 & 1112.54 \\
\hline MW8S & 1098.53 & 3.31 & 1095.22 & 3.06 & 1095.47 & 2.20 & 1096.33 & 0.70 & 1097.83 & 0.61 & 1097.92 \\
\hline MW9S & 1118.31 & 19.29 & 1099.02 & 13.35 & 1104.96 & 14.00 & 1104.31 & 16.58 & 1101.73 & 16.76 & 1101.55 \\
\hline MW10S & 1110.78 & 11.62 & 1099.16 & 6.32 & 1104.46 & 6.95 & 1103.83 & 9.82 & 1100.96 & 9.83 & 1100.95 \\
\hline MW11S & 1133.08 & 36.45 & 1096.63 & 30.90 & 1102.18 & 31.55 & 1101.53 & 29.90 & 1103.18 & 29.81 & 1103.27 \\
\hline
\end{tabular}

\begin{tabular}{|c|c|c|c|c|c|}
\hline \multirow[b]{2}{*}{ Well } & \multirow[b]{2}{*}{$\begin{array}{l}\text { Top of Casing } \\
\text { Elevation } \\
\text { (ft AMSL) }\end{array}$} & \multicolumn{2}{|c|}{ September 19, 2008} & \multicolumn{2}{|c|}{ October 20-21, 2008} \\
\hline & & $\begin{array}{l}\text { Depth to } \\
\text { Water } \\
\text { (ft BGL) }\end{array}$ & $\begin{array}{l}\text { Groundwater } \\
\text { Elevation } \\
\text { (ft AMSL) }\end{array}$ & $\begin{array}{l}\text { Depth to } \\
\text { Water } \\
\text { (ft BGL) }\end{array}$ & $\begin{array}{c}\text { Groundwate } \\
\text { Elevation } \\
\text { (ft AMSL) }\end{array}$ \\
\hline MW1S & 1124.68 & 23.51 & 1101.17 & 25.8 & 1098.88 \\
\hline MW1D & 1124.63 & 30.01 & 1094.62 & 30.4 & 1094.23 \\
\hline MW2S & 1137.07 & 32.20 & 1104.87 & 33.7 & 1103.37 \\
\hline MW3S & 1135.76 & 24.42 & 1111.34 & 27.0 & 1108.76 \\
\hline MW4S & 1143.61 & 34.62 & 1108.99 & 36.7 & 1106.91 \\
\hline MW5S & 1122.21 & 21.21 & 1101.00 & 22.5 & 1099.71 \\
\hline MW6S & 1090.97 & 5.13 & 1085.84 & 5.7 & 1085.27 \\
\hline MW7S & 1119.86 & 15.38 & 1104.48 & 17.2 & 1102.66 \\
\hline MW8S & 1098.53 & 2.98 & 1095.55 & 3.6 & 1094.93 \\
\hline MW9S & 1118.31 & 20.46 & 1097.85 & 21.5 & 1096.81 \\
\hline MW10S & 1110.78 & 12.84 & 1097.94 & 13.7 & 1097.08 \\
\hline MW11s & 1133.08 & 35.67 & 1097.41 & 37.1 & 1095.98 \\
\hline
\end{tabular}


TABLE 3.2 Results of analyses at the AGEM Laboratory for volatile organic compounds in groundwater samples collected at Morrill, October 2003 to October 2008. Shading indicates sample collection with the low-flow procedure. ${ }^{a}$

\begin{tabular}{|c|c|c|c|c|c|c|c|c|c|c|c|c|}
\hline \multirow[b]{2}{*}{ Location } & \multirow[b]{2}{*}{$\begin{array}{l}\text { Screen } \\
\text { Interval } \\
\text { (ft BGL) }\end{array}$} & \multirow[b]{2}{*}{$\begin{array}{l}\text { Sample } \\
\text { Date }\end{array}$} & \multirow[b]{2}{*}{$\begin{array}{c}\text { Depth to } \\
\text { Water } \\
\text { (ft TOC) }^{\mathrm{b}}\end{array}$} & \multirow[b]{2}{*}{$\begin{array}{l}\text { Depth of } \\
\text { Well } \\
\text { (ft TOC) }\end{array}$} & \multirow[b]{2}{*}{$\begin{array}{l}\text { Volume } \\
\text { Purged }\end{array}$} & \multirow[b]{2}{*}{$\begin{array}{l}\text { Volume } \\
\text { Units }\end{array}$} & \multirow{2}{*}{$\begin{array}{l}\text { Pump } \\
\text { Intake } \\
\text { Position } \\
\text { (ft BGL) }\end{array}$} & \multicolumn{3}{|c|}{ Concentration $(\mu \mathrm{g} / \mathrm{L})$} & \multirow[b]{2}{*}{ Comment } & \multirow[b]{2}{*}{ Sample } \\
\hline & & & & & & & & $\begin{array}{c}\text { Carbon } \\
\text { Tetrachloride }\end{array}$ & Chloroform & $\begin{array}{l}\text { Methylene } \\
\text { Chloride }\end{array}$ & & \\
\hline \multirow[t]{14}{*}{ MW1S } & $11-51$ & $10 / 23 / 03$ & 30.36 & 54.04 & 70 & gal & - & 33 & 1.6 & $N D^{c}$ & - & MRMW1S-W-16422 \\
\hline & & $6 / 2 / 04$ & 26.97 & 53.94 & 53 & gal & - & 19 & 0.9 & ND & - & MRMW1S-W-16461 \\
\hline & & $9 / 13 / 05$ & 24.16 & 53.9 & 57 & gal & - & 35 & 1.7 & ND & _- & MRMW1S-W-19259 \\
\hline & & $3 / 22 / 06$ & 29 & 53.95 & 48 & gal & - & 40 & 1.8 & ND & _- & MRMW1S-W-20008 \\
\hline & & 9/20/06 & 26.82 & 53.97 & 55 & gal & - & 23 & $0.9 \mathrm{~J}^{\mathrm{d}}$ & ND & _- & MRMW1S-W-22495 \\
\hline & & $3 / 21 / 07$ & 25.8 & 53.98 & 55 & gal & - & 23 & 1.1 & ND & _- & MRMW1S-W-16488 \\
\hline & & $10 / 1 / 07$ & 21.65 & 53.95 & 63 & gal & - & 56 & 2.7 & ND & - & MRMW1S-W-16595 \\
\hline & & $4 / 14 / 08$ & 16.2 & 54 & 5.5 & L & - & $0.3 \mathrm{~J}$ & ND & ND & April monitoring. & MRMW1S-W-23230 \\
\hline & & $4 / 22 / 08$ & 16 & 54 & 6.25 & L & - & $0.2 \mathrm{~J}$ & ND & ND & $\begin{array}{l}\text { Confirm low carbon } \\
\text { tetrachloride. }\end{array}$ & MRMW1S-W-23259 \\
\hline & & $5 / 1 / 08$ & - & - & 3.24 & L & 22 & ND & ND & ND & Vary pump intake. & MRMW1S-22-W-23275 \\
\hline & & $5 / 1 / 08$ & - & - & 3.24 & $\mathrm{~L}$ & 27 & ND & ND & ND & Vary pump intake. & MRMW1S-27-W-23276 \\
\hline & & $5 / 1 / 08$ & _ & _ & 4.25 & $\mathrm{~L}$ & 48 & $0.3 \mathrm{~J}$ & ND & ND & Vary pump intake. & MRMW1S-48-W-23277 \\
\hline & & $10 / 20 / 08$ & 25.8 & 54 & 6 & L & 31 & $0.7 \mathrm{~J}$ & ND & ND & Low flow. & MRMW1S-W-27620 \\
\hline & & $10 / 21 / 08$ & - & - & 57 & gal & - & 35 & 1.8 & ND & $\begin{array}{l}\text { Full purge (three } \\
\text { well volumes. }\end{array}$ & MRMW1S-W-27649 \\
\hline \multirow[t]{9}{*}{ MW1D } & $63-88$ & $10 / 22 / 03$ & 28.39 & 88.5 & 92 & gal & - & ND & ND & ND & - & MRMW1D-W-16421 \\
\hline & & $6 / 2 / 04$ & 26.82 & 88.6 & 140 & gal & - & ND & ND & ND & - & MRMW1D-W-16458 \\
\hline & & $9 / 13 / 05$ & 23.72 & 88.6 & 200 & gal & - & ND & ND & ND & - & MRMW1D-W-16518 \\
\hline & & $3 / 19 / 06$ & 26.85 & 88.6 & 112 & gal & - & ND & ND & $0.4 \mathrm{~J} \mathrm{~B}^{\mathrm{e}}$ & _- & MRMW1D-W-19986 \\
\hline & & 9/20/06 & 25.52 & 88.8 & 125 & gal & - & ND & ND & ND & - & MRMW1D-W-16532 \\
\hline & & $3 / 21 / 07$ & 25.79 & 88.8 & 125 & gal & - & ND & ND & ND & _- & MRMW1D-W-16487 \\
\hline & & $10 / 1 / 07$ & 22.75 & 89.38 & 130 & gal & - & ND & ND & ND & - & MRMW1D-W-16596 \\
\hline & & $4 / 14 / 08$ & 29.51 & 89 & 6 & L & - & ND & ND & ND & - & MRMW1D-W-23231 \\
\hline & & $10 / 20 / 08$ & 30.4 & 89 & 7 & L & 75.5 & ND & ND & ND & - & MRMW1D-W-27621 \\
\hline MW2S & $13-53$ & $10 / 22 / 03$ & 42.21 & 53.35 & & & & ND & ND & ND & $\begin{array}{l}\text { Purged dry, then } \\
\text { sampled. }\end{array}$ & MRMW02-W-16419 \\
\hline \multirow[t]{9}{*}{ MW2S } & $13-53$ & $6 / 2 / 04$ & 37.44 & 53.3 & 31 & gal & - & ND & ND & ND & - & MRMW2S-W-16459 \\
\hline & & $9 / 14 / 05$ & 33.68 & 53.33 & 38 & gal & - & ND & ND & ND & - & MRMW2S-W-19264 \\
\hline & & $3 / 21 / 06$ & 40.87 & 53.32 & 27 & gal & _- & ND & ND & ND & _- & MRMW2S-W-19992 \\
\hline & & 9/18/06 & 36.53 & 53.3 & 28 & gal & - & ND & ND & ND & - & MRMW2S-W-22488 \\
\hline & & $3 / 22 / 07$ & 35.77 & 53.3 & 35 & gal & _- & ND & ND & ND & _- & MRMW2S-W-16559 \\
\hline & & $10 / 3 / 07$ & 31.17 & 53.4 & 44 & gal & - & ND & ND & ND & - & MRMW2S-W-16587 \\
\hline & & $4 / 15 / 08$ & 23.55 & 53.4 & 2.22 & L & - & ND & ND & ND & - & MRMW2S-W-23232 \\
\hline & & $10 / 21 / 08$ & 33.7 & 53.5 & 5 & L & 33 & ND & ND & ND & Low flow. & MRMW2S-W-27622 \\
\hline & & $10 / 21 / 08$ & - & - & 39 & gal & - & ND & ND & ND & $\begin{array}{l}\text { Full purge (three } \\
\text { well volumes). }\end{array}$ & MRMW2S-W-27652 \\
\hline
\end{tabular}


TABLE 3.2 (Cont.)

\begin{tabular}{|c|c|c|c|c|c|c|c|c|c|c|c|c|}
\hline \multirow[b]{2}{*}{ Location } & \multirow[b]{2}{*}{$\begin{array}{l}\text { Screen } \\
\text { Interval } \\
\text { (ft BGL) }\end{array}$} & \multirow[b]{2}{*}{$\begin{array}{l}\text { Sample } \\
\text { Date }\end{array}$} & \multirow[b]{2}{*}{$\begin{array}{l}\text { Depth to } \\
\text { Water } \\
\text { (ft TOC) }^{\mathrm{b}}\end{array}$} & \multirow[b]{2}{*}{$\begin{array}{l}\text { Depth of } \\
\text { Well } \\
\text { (ft TOC) }\end{array}$} & \multirow[b]{2}{*}{$\begin{array}{l}\text { Volume } \\
\text { Purged }\end{array}$} & \multirow[b]{2}{*}{$\begin{array}{l}\text { Volume } \\
\text { Units }\end{array}$} & \multirow{2}{*}{$\begin{array}{l}\text { Pump } \\
\text { Intake } \\
\text { Position } \\
\text { (ft BGL) }\end{array}$} & \multicolumn{3}{|c|}{ Concentration $(\mu \mathrm{g} / \mathrm{L})$} & \multirow[b]{2}{*}{ Comment } & \multirow[b]{2}{*}{ Sample } \\
\hline & & & & & & & & $\begin{array}{c}\text { Carbon } \\
\text { Tetrachloride }\end{array}$ & Chloroform & $\begin{array}{l}\text { Methylene } \\
\text { Chloride }\end{array}$ & & \\
\hline \multirow[t]{14}{*}{ MW3S } & $18-48$ & $10 / 23 / 03$ & 36.47 & 47.79 & 73 & gal & - & 89 & 2.7 & ND & - & MRMW03-W-16423 \\
\hline & & $6 / 2 / 04$ & 30.67 & 47.5 & 34 & gal & - & 110 & 3.2 & ND & _- & MRMW3S-W-16462 \\
\hline & & 9/13/05 & 25.6 & 47.6 & 50 & gal & - & 101 & 3.2 & ND & - & MRMW3S-W-19261 \\
\hline & & $3 / 23 / 06$ & 35.62 & 47.74 & 28 & gal & - & 91 & 2.6 & ND & - & MRMW3S-W-19994 \\
\hline & & $9 / 20 / 06$ & 29.44 & 47.75 & 22 & gal & - & 49 & 1.5 & ND & - & MRMW3S-W-22496 \\
\hline & & $3 / 22 / 07$ & 26.19 & 47.75 & 45 & gal & - & 84 & 2.3 & ND & _- & MRMW3S-W-16563 \\
\hline & & $10 / 3 / 07$ & 22.7 & 47.9 & 50 & gal & - & 61 & 2.0 & ND & - & MRMW3S-W-16585 \\
\hline & & $4 / 14 / 08$ & 16.95 & 47.8 & 3.31 & $\mathrm{~L}$ & - & 8.2 & $0.4 \mathrm{~J}$ & ND & April monitoring. & MRMW3S-W-23233 \\
\hline & & $4 / 22 / 08$ & 15.8 & 47.8 & 6.5 & L & - & $0.7 \mathrm{~J}$ & ND & ND & $\begin{array}{l}\text { Confirm low carbon } \\
\text { tetrachloride. }\end{array}$ & MRMW3S-W-23260 \\
\hline & & $5 / 1 / 08$ & _- & - & 2.62 & L & 26 & $0.4 \mathrm{~J}$ & ND & ND & Vary pump intake. & MRMW3S-26-W-23269 \\
\hline & & $5 / 1 / 08$ & - & - & 2.75 & L & 38 & $0.4 \mathrm{~J}$ & ND & ND & Vary pump intake. & MRMW3S-38-W-23270 \\
\hline & & $5 / 1 / 08$ & _- & - & 3.17 & L & 45 & $0.5 \mathrm{~J}$ & ND & ND & Vary pump intake. & MRMW3S-45-W-23271 \\
\hline & & $10 / 21 / 08$ & 27 & 47.8 & 4.2 & L & 33 & 55 & 1.4 & ND & Low flow. & MRMW3S-W-27623 \\
\hline & & $10 / 21 / 08$ & - & - & 41 & gal & - & 63 & 1.6 & ND & $\begin{array}{l}\text { Full purge (three } \\
\text { well volumes). }\end{array}$ & MRMW3S-W-27650 \\
\hline \multirow[t]{10}{*}{ MW4S } & $17-47$ & $10 / 21 / 03$ & 46.4 & 47.8 & & - & & ND & ND & ND & $\begin{array}{l}\text { Purged dry, then } \\
\text { sampled. }\end{array}$ & MRMW04-W-16418 \\
\hline & & $6 / 4 / 04$ & 43.21 & 47.8 & 10 & gal & - & ND & ND & ND & - & MRMW4S-W-16470 \\
\hline & & $9 / 14 / 05$ & 36.21 & 47.81 & 8 & gal & - & ND & ND & ND & - & MRMW4S-W-19262 \\
\hline & & $3 / 21 / 06$ & 44.55 & 47.72 & 6 & gal & - & ND & ND & ND & _- & MRMW4S-W-19993 \\
\hline & & $9 / 18 / 06$ & 41.56 & 47.75 & 5.25 & gal & - & ND & ND & ND & - & MRMW4S-W-22487 \\
\hline & & $3 / 22 / 07$ & 38.74 & 47.75 & 6 & gal & - & ND & ND & ND & _- & MRMW4S-W-16562 \\
\hline & & $10 / 3 / 07$ & 31.11 & 47.73 & 30 & gal & - & $0.5 \mathrm{~J} \mathrm{R}^{\dagger}$ & ND & ND & - & MRMW4S-W-16586 \\
\hline & & $1 / 11 / 08$ & - & _ & - & - & - & ND & ND & ND & _- & MOMW4S-W-011108 \\
\hline & & $4 / 14 / 08$ & 26.32 & 47.85 & 2.48 & L & - & ND & ND & ND & - & MRMW4S-W-23234 \\
\hline & & $10 / 20 / 08$ & 36.7 & 47.8 & 8 & gal & - & ND & ND & ND & - & MRMW4S-W-27624 \\
\hline \multirow[t]{11}{*}{ MW5S } & $15-55$ & $10 / 22 / 03$ & 31.4 & 55.72 & 48 & gal & - & 5.8 & ND & ND & - & MRMW05-W-16420 \\
\hline & & $6 / 2 / 04$ & 26.33 & 55.65 & $>57$ & gal & - & 7.0 & ND & ND & - & MRMW5S-W-16460 \\
\hline & & 9/13/05 & 22.66 & 54.2 & 75 & gal & - & 6.3 & $0.2 \mathrm{~J}$ & ND & - & MRMW5S-W-19260 \\
\hline & & $3 / 22 / 06$ & 28.64 & 54.51 & 50 & gal & - & 7.3 & $0.2 \mathrm{~J}$ & ND & - & MRMW5S-W-19996 \\
\hline & & $9 / 20 / 06$ & 25.43 & 54.63 & 52 & gal & - & 6.4 & $0.3 \mathrm{~J}$ & ND & - & MRMW5S-W-22493 \\
\hline & & $3 / 22 / 07$ & 25.14 & 54.56 & 58 & gal & - & 6.5 & $0.4 \mathrm{~J}$ & ND & - & MRMW5S-W-16569 \\
\hline & & $10 / 3 / 07$ & 19.55 & 54.65 & 68 & gal & - & 4.0 & $0.3 \mathrm{~J}$ & ND & - & MRMW5S-W-16588 \\
\hline & & $4 / 14 / 08$ & 11.2 & 54.6 & 6 & L & - & ND & ND & ND & April monitoring. & MRMW5S-W-23235 \\
\hline & & $4 / 23 / 08$ & 11.3 & 54.6 & 6.5 & L & - & ND & ND & ND & $\begin{array}{l}\text { Confirm low carbon } \\
\text { tetrachloride. }\end{array}$ & MRMW5S-W-23266 \\
\hline & & $5 / 1 / 08$ & - & - & 3.7 & L & 20 & ND & ND & ND & Vary pump intake. & MRMW5S-20-W-23272 \\
\hline & & $5 / 1 / 08$ & - & - & 3.4 & L & 28 & ND & ND & ND & Vary pump intake. & MRMW5S-28-W-23273 \\
\hline
\end{tabular}


TABLE 3.2 (Cont.)

\begin{tabular}{|c|c|c|c|c|c|c|c|c|c|c|c|c|}
\hline \multirow[b]{2}{*}{ Location } & \multirow[b]{2}{*}{$\begin{array}{l}\text { Screen } \\
\text { Interval } \\
\text { (ft BGL) }\end{array}$} & \multirow[b]{2}{*}{$\begin{array}{l}\text { Sample } \\
\text { Date }\end{array}$} & \multirow[b]{2}{*}{$\begin{array}{l}\text { Depth to } \\
\text { Water } \\
\text { (ft TOC) }\end{array}$} & \multirow[b]{2}{*}{$\begin{array}{l}\text { Depth of } \\
\text { Well } \\
\text { (ft TOC) }\end{array}$} & \multirow[b]{2}{*}{$\begin{array}{l}\text { Volume } \\
\text { Purged }\end{array}$} & \multirow[b]{2}{*}{$\begin{array}{l}\text { Volume } \\
\text { Units }\end{array}$} & \multirow{2}{*}{$\begin{array}{l}\text { Pump } \\
\text { Intake } \\
\text { Position } \\
\text { (ft BGL) }\end{array}$} & \multicolumn{3}{|c|}{ Concentration $(\mu \mathrm{g} / \mathrm{L})$} & \multirow[b]{2}{*}{ Comment } & \multirow[b]{2}{*}{ Sample } \\
\hline & & & & & & & & $\begin{array}{c}\text { Carbon } \\
\text { Tetrachloride }\end{array}$ & Chloroform & $\begin{array}{l}\text { Methylene } \\
\text { Chloride }\end{array}$ & & \\
\hline MW5S & $15-55$ & $\begin{array}{r}5 / 1 / 08 \\
10 / 21 / 08\end{array}$ & $\overline{22.5}$ & $\overline{54.6}$ & $\begin{array}{l}4 \\
7\end{array}$ & $\begin{array}{l}\mathrm{L} \\
\mathrm{L}\end{array}$ & $\begin{array}{l}52 \\
35\end{array}$ & $\begin{array}{l}\text { ND } \\
1.7\end{array}$ & $\begin{array}{l}\text { ND } \\
\text { ND }\end{array}$ & $\begin{array}{l}\text { ND } \\
\text { ND }\end{array}$ & Vary pump intake. & $\begin{array}{l}\text { MRMW5S-52-W-23274 } \\
\text { MRMW5S-W-27625 }\end{array}$ \\
\hline MW6S & $10-25$ & $\begin{array}{r}6 / 3 / 04 \\
9 / 14 / 05 \\
3 / 20 / 06 \\
9 / 18 / 06 \\
3 / 21 / 07 \\
10 / 2 / 07 \\
4 / 15 / 08 \\
10 / 20 / 08\end{array}$ & $\begin{array}{l}3.34 \\
4.7 \\
5.35 \\
5.48 \\
5.42 \\
5 \\
5.15 \\
5.7\end{array}$ & $\begin{array}{l}26.9 \\
26.9 \\
26.91 \\
26.92 \\
26.92 \\
26.9 \\
26.9 \\
26.9\end{array}$ & $\begin{array}{c}45 \\
43 \\
43 \\
27 \\
30 \\
31 \\
2.5 \\
5\end{array}$ & $\begin{array}{l}\text { gal } \\
\text { gal } \\
\text { gal } \\
\text { gal } \\
\text { gal } \\
\text { gal } \\
\text { L } \\
\text { L }\end{array}$ & $\begin{array}{c}- \\
- \\
- \\
- \\
- \\
- \\
- \\
17.5\end{array}$ & $\begin{array}{l}\text { ND } \\
\text { ND } \\
\text { ND } \\
\text { ND } \\
\text { ND } \\
\text { ND } \\
\text { ND } \\
\text { ND }\end{array}$ & $\begin{array}{l}\text { ND } \\
\text { ND } \\
\text { ND } \\
\text { ND } \\
\text { ND } \\
\text { ND } \\
\text { ND } \\
\text { ND }\end{array}$ & $\begin{array}{l}\text { ND } \\
\text { ND } \\
\text { ND } \\
\text { ND } \\
\text { ND } \\
\text { ND } \\
\text { ND } \\
\text { ND }\end{array}$ & $\begin{array}{l}- \\
- \\
- \\
- \\
- \\
- \\
- \\
-\end{array}$ & $\begin{array}{l}\text { MRMW6S-W-16465 } \\
\text { MRMW6S-W-19263 } \\
\text { MRMW6S-W-19990 } \\
\text { MRMW6S-W-22486 } \\
\text { MRMW6S-W-16486 } \\
\text { MRMW6S-W-16583 } \\
\text { MRMW6S-W-23236 } \\
\text { MRMW6S-W-27626 }\end{array}$ \\
\hline MW7S & $20-45$ & $\begin{array}{r}6 / 3 / 04 \\
9 / 12 / 05 \\
3 / 22 / 06 \\
9 / 19 / 06 \\
3 / 20 / 07 \\
10 / 1 / 07 \\
4 / 14 / 08 \\
4 / 23 / 08 \\
10 / 20 / 08\end{array}$ & $\begin{array}{c}26.68 \\
17.57 \\
22.45 \\
20.94 \\
18.01 \\
12.4 \\
7.72 \\
7.8 \\
17.2\end{array}$ & $\begin{array}{l}46.98 \\
46.94 \\
46.98 \\
47.02 \\
47.02 \\
47 \\
47 \\
47 \\
47\end{array}$ & $\begin{array}{l}40 \\
55 \\
48 \\
56 \\
50 \\
70 \\
1.82 \\
11 \\
6.3\end{array}$ & $\begin{array}{l}\text { gal } \\
\text { gal } \\
\text { gal } \\
\text { gal } \\
\text { gal } \\
\text { gal } \\
\text { L } \\
\text { L } \\
\text { L }\end{array}$ & $\begin{array}{l}- \\
- \\
- \\
- \\
- \\
- \\
- \\
- \\
32.5\end{array}$ & $\begin{array}{c}18 \\
43 \\
21 \\
38 \\
16 \\
8.1 \\
10 \\
8.3 \\
7.9\end{array}$ & $\begin{array}{l}\text { ND } \\
1.1 \\
0.4 \mathrm{~J} \\
0.7 \mathrm{~J} \\
0.4 \mathrm{~J} \\
0.2 \mathrm{~J} \\
0.3 \mathrm{~J} \\
0.2 \mathrm{~J} \\
\mathrm{ND}\end{array}$ & $\begin{array}{l}\text { ND } \\
\text { ND } \\
\text { ND } \\
\text { ND } \\
\text { ND } \\
\text { ND } \\
\text { ND } \\
\text { ND } \\
\text { ND }\end{array}$ & $\begin{array}{l}- \\
- \\
- \\
- \\
- \\
- \\
- \\
- \\
-\end{array}$ & $\begin{array}{l}\text { MRMW7S-W-16466 } \\
\text { MRMW7S-W-19258 } \\
\text { MRMW7S-W-20000 } \\
\text { MRMW7S-W-22490 } \\
\text { MRMW7S-W-16481 } \\
\text { MRMW7S-W-16581 } \\
\text { MRMW7S-W-23237 } \\
\text { MRMW7S-W-23265 } \\
\text { MRMW7S-W-27627 }\end{array}$ \\
\hline MW8S & $10-25$ & $\begin{array}{r}6 / 3 / 04 \\
9 / 14 / 05 \\
3 / 20 / 06 \\
9 / 19 / 06 \\
3 / 20 / 07 \\
10 / 2 / 07 \\
4 / 15 / 08 \\
10 / 20 / 08\end{array}$ & $\begin{array}{l}3.7 \\
4.02 \\
4.57 \\
4.76 \\
2.63 \\
2.2 \\
0.7 \\
3.6\end{array}$ & $\begin{array}{l}26.75 \\
26.82 \\
26.41 \\
26.83 \\
26.82 \\
26.8 \\
26.8 \\
26.8\end{array}$ & $\begin{array}{l}45 \\
57 \\
43 \\
45 \\
49 \\
48 \\
5.5 \\
8\end{array}$ & $\begin{array}{l}\text { gal } \\
\text { gal } \\
\text { gal } \\
\text { gal } \\
\text { gal } \\
\text { gal } \\
\text { L } \\
\text { L }\end{array}$ & $\begin{array}{l}- \\
- \\
- \\
- \\
- \\
- \\
- \\
17.5\end{array}$ & $\begin{array}{l}\text { ND } \\
0.9 \mathrm{~J} \\
0.6 \mathrm{~J} \\
1.3 \\
0.6 \mathrm{~J} \\
0.8 \mathrm{~J} \\
1.1 \\
1.3\end{array}$ & $\begin{array}{l}\text { ND } \\
\text { ND } \\
\text { ND } \\
\text { ND } \\
\text { ND } \\
\text { ND } \\
\text { ND } \\
\text { ND }\end{array}$ & $\begin{array}{l}\text { ND } \\
\text { ND } \\
0.4 \mathrm{~J} \mathrm{~B} \\
\text { ND } \\
\text { ND } \\
\text { ND } \\
\text { ND } \\
\text { ND }\end{array}$ & $\begin{array}{l}- \\
- \\
- \\
- \\
- \\
- \\
- \\
-\end{array}$ & $\begin{array}{l}\text { MRMW8S-W-16464 } \\
\text { MRMW8S-W-19265 } \\
\text { MRMW8S-W-19991 } \\
\text { MRMW8S-W-22492 } \\
\text { MRMW8S-W-16483 } \\
\text { MRMW8S-W-16584 } \\
\text { MRMW8S-W-23238 } \\
\text { MRMW8S-W-27628 }\end{array}$ \\
\hline MW9S & $38.83-53.83$ & $\begin{array}{r}3 / 22 / 06 \\
9 / 19 / 06 \\
3 / 20 / 07 \\
10 / 1 / 07 \\
4 / 14 / 08 \\
10 / 20 / 08\end{array}$ & $\begin{array}{l}20.2 \\
18.87 \\
16.69 \\
14 \\
16.58 \\
21.5\end{array}$ & $\begin{array}{l}58.62 \\
59 \\
59 \\
58.6 \\
58.63 \\
58.5\end{array}$ & $\begin{array}{c}20 \\
22 \\
22 \\
23 \\
2.29 \\
11\end{array}$ & $\begin{array}{l}\text { gal } \\
\text { gal } \\
\text { gal } \\
\text { gal } \\
\text { L } \\
\text { L }\end{array}$ & $\begin{array}{c}- \\
- \\
- \\
- \\
- \\
46.33\end{array}$ & $\begin{array}{l}\text { ND } \\
\text { ND } \\
\text { ND } \\
\text { ND } \\
0.8 \mathrm{~J} \\
1.1\end{array}$ & $\begin{array}{l}\text { ND } \\
\text { ND } \\
\text { ND } \\
\text { ND } \\
\text { ND } \\
\text { ND }\end{array}$ & $\begin{array}{l}\text { ND } \\
\text { ND } \\
\text { ND } \\
\text { ND } \\
\text { ND } \\
\text { ND }\end{array}$ & $\begin{array}{l}- \\
- \\
- \\
- \\
- \\
-\end{array}$ & $\begin{array}{l}\text { MRMW9S-W-20004 } \\
\text { MRMW9S-W-22494 } \\
\text { MRMW9S-W-16480 } \\
\text { MRMW9S-W-16582 } \\
\text { MRMW9S-W-23239 } \\
\text { MRMW9S-W-27629 }\end{array}$ \\
\hline
\end{tabular}


TABLE 3.2 (Cont.)

\begin{tabular}{|c|c|c|c|c|c|c|c|c|c|c|c|c|}
\hline \multirow[b]{2}{*}{ Location } & \multirow[b]{2}{*}{$\begin{array}{l}\text { Screen } \\
\text { Interval } \\
\text { (ft BGL) }\end{array}$} & \multirow[b]{2}{*}{$\begin{array}{l}\text { Sample } \\
\text { Date }\end{array}$} & \multirow[b]{2}{*}{$\begin{array}{l}\text { Depth to } \\
\text { Water } \\
\text { (ft TOC) }\end{array}$} & \multirow[b]{2}{*}{$\begin{array}{l}\text { Depth of } \\
\text { Well } \\
\text { (ft TOC) }\end{array}$} & \multirow[b]{2}{*}{$\begin{array}{l}\text { Volume } \\
\text { Purged }\end{array}$} & \multirow[b]{2}{*}{$\begin{array}{l}\text { Volume } \\
\text { Units }\end{array}$} & \multirow{2}{*}{$\begin{array}{l}\text { Pump } \\
\text { Intake } \\
\text { Position } \\
\text { (ft BGL) }\end{array}$} & \multicolumn{3}{|c|}{ Concentration $(\mu \mathrm{g} / \mathrm{L})$} & \multirow[b]{2}{*}{ Comment } & \multirow[b]{2}{*}{ Sample } \\
\hline & & & & & & & & $\begin{array}{c}\text { Carbon } \\
\text { Tetrachloride }\end{array}$ & Chloroform & $\begin{array}{l}\text { Methylene } \\
\text { Chloride }\end{array}$ & & \\
\hline \multirow[t]{6}{*}{ MW10S } & $30-45$ & $3 / 21 / 06$ & 12.3 & 49.6 & 19 & gal & - & ND & ND & ND & - & MRMW10S-W-19999 \\
\hline & & $9 / 18 / 06$ & 11.08 & 49.61 & 20 & gal & - & ND & ND & ND & - & MRMW10S-W-22489 \\
\hline & & $3 / 21 / 07$ & 10.77 & 49.61 & 20 & gal & - & ND & ND & ND & - & MRMW10S-W-16485 \\
\hline & & $10 / 1 / 07$ & 6.95 & 49.65 & 20 & gal & - & ND & ND & ND & _- & MRMW10S-W-16593 \\
\hline & & $4 / 14 / 08$ & 9.82 & 49.7 & 1.9 & $\mathrm{~L}$ & - & ND & ND & ND & - & MRMW10S-W-23240 \\
\hline & & $10 / 20 / 08$ & 13.7 & 49.7 & 5.4 & L & 37.5 & ND & ND & ND & - & MRMW10S-W-27630 \\
\hline \multirow[t]{8}{*}{ MW11S } & $53-68$ & $3 / 22 / 06$ & 35.2 & 72.5 & 20 & gal & - & 39 & $0.9 \mathrm{~J}$ & ND & - & MRMW11S-W-20001 \\
\hline & & 9/19/06 & 36 & 73.14 & 20 & gal & _- & 53 & 1.0 & ND & _- & MRMW11S-W-22491 \\
\hline & & $3 / 20 / 07$ & 34.65 & 73.14 & 20 & gal & - & 37 & $0.8 \mathrm{~J}$ & ND & - & MRMW11S-W-16479 \\
\hline & & $10 / 1 / 07$ & 31.55 & 73 & 20 & gal & - & 54 & 1.2 & ND & - & MRMW11S-W-16594 \\
\hline & & $4 / 15 / 08$ & 29.9 & 72.7 & 5.5 & L & - & 35 & $0.8 \mathrm{~J}$ & ND & April monitoring. & MRMW11S-W-23241 \\
\hline & & $4 / 22 / 08$ & 30.2 & 72.7 & 7.2 & L & - & 42 & $0.9 \mathrm{~J}$ & ND & $\begin{array}{l}\text { Confirm low carbon } \\
\text { tetrachloride. }\end{array}$ & MRMW11S-W-23261 \\
\hline & & $10 / 20 / 08$ & 37.1 & 72.7 & 9 & L & 60.5 & 42 & $0.9 \mathrm{~J}$ & ND & Low flow. & MRMW11S-W-27631 \\
\hline & & $10 / 21 / 08$ & - & - & 18 & gal & - & 45 & $0.9 \mathrm{~J}$ & ND & $\begin{array}{l}\text { Full purge (three } \\
\text { well volumes). }\end{array}$ & MRMW11S-W-27651 \\
\hline \multirow[t]{9}{*}{ Isch } & _ & $2 / 19 / 04$ & - & - & Pump ${ }^{g}$ & - & _ & ND & ND & ND & _ & MRJR-W-16502 \\
\hline & & $9 / 14 / 05$ & _- & _- & Pump & _- & _- & ND & ND & ND & _- & MRPRISCH-W-16513 \\
\hline & & $3 / 23 / 06$ & - & - & 20 & gal & - & ND & ND & ND & - & MRISCH-W-19989 \\
\hline & & 9/19/06 & _- & _- & Pump & - & _- & ND & ND & ND & _- & MRISCH-W-16531 \\
\hline & & $3 / 22 / 07$ & - & - & Pump & - & - & ND & ND & ND & - & MRISCH-W-16564 \\
\hline & & $10 / 3 / 07$ & _- & - & Pump & - & _- & ND & ND & ND & - & MRISCH-W-16590 \\
\hline & & $4 / 15 / 08$ & - & - & Pump & - & - & $0.4 \mathrm{~J}$ & ND & ND & - & MRISCH-W-23242 \\
\hline & & $4 / 22 / 08$ & - & - & Pump & - & - & ND & ND & ND & - & MRISCH-W-23262 \\
\hline & & $10 / 21 / 08$ & - & - & Pump & - & - & ND & ND & ND & - & MRISCH-W-27632 \\
\hline \multirow[t]{8}{*}{ Rilinger } & - & $6 / 4 / 04$ & - & - & Pump & - & - & ND & ND & ND & - & MRPRIVRIL-W-16471 \\
\hline & & 9/14/05 & - & - & Pump & - & - & 2.6 & $0.1 \mathrm{~J}$ & ND & - & MRPRILL-W-16512 \\
\hline & & $3 / 19 / 06$ & - & - & Pump & - & - & ND & ND & $0.4 \mathrm{~J} \mathrm{~B}$ & - & $\begin{array}{l}\text { MRRILINGER-W- } \\
19988\end{array}$ \\
\hline & & 9/19/06 & _- & _- & Pump & _- & _- & ND & ND & ND & _- & MRRILI-W-16530 \\
\hline & & $3 / 29 / 07$ & - & - & Pump & - & - & 1.3 & 1.1 & ND & - & $\begin{array}{l}\text { MRRILINGER-W- } \\
16561\end{array}$ \\
\hline & & $10 / 3 / 07$ & - & - & Pump & - & - & 13 & $0.4 \mathrm{~J}$ & ND & $\begin{array}{l}\text { Well reactivated } \\
\text { shortly before } \\
\text { sampling. }\end{array}$ & $\begin{array}{l}\text { MRRILINGER-W- } \\
16591\end{array}$ \\
\hline & & $10 / 8 / 07$ & - & - & Pump & - & - & $0.4 \mathrm{~J}$ & ND & ND & Verify $10 / 3 / 07$ result. & $\begin{array}{l}\text { MRRILINGER-W- } \\
16592\end{array}$ \\
\hline & & $1 / 11 / 08$ & - & - & Pump & - & - & 6.2 & $0.5 \mathrm{~J}$ & ND & Verify $10 / 3 / 07$ result. & MORIL-W-11108 \\
\hline
\end{tabular}


TABLE 3.2 (Cont.)

\begin{tabular}{|c|c|c|c|c|c|c|c|c|c|c|c|c|}
\hline \multirow[b]{2}{*}{ Location } & \multirow[b]{2}{*}{$\begin{array}{l}\text { Screen } \\
\text { Interval } \\
\text { (ft BGL) }\end{array}$} & \multirow[b]{2}{*}{$\begin{array}{l}\text { Sample } \\
\text { Date }\end{array}$} & \multirow[b]{2}{*}{$\begin{array}{l}\text { Depth to } \\
\text { Water } \\
\left(\mathrm{ft} \mathrm{TOC}^{\mathrm{b}}{ }^{\mathrm{s}}\right.\end{array}$} & \multirow[b]{2}{*}{$\begin{array}{l}\text { Depth of } \\
\text { Well } \\
\text { (ft TOC) }\end{array}$} & \multirow[b]{2}{*}{$\begin{array}{l}\text { Volume } \\
\text { Purged }\end{array}$} & \multirow[b]{2}{*}{$\begin{array}{l}\text { Volume } \\
\text { Units }\end{array}$} & \multirow{2}{*}{$\begin{array}{l}\text { Pump } \\
\text { Intake } \\
\text { Position } \\
\text { (ft BGL) }\end{array}$} & \multicolumn{3}{|c|}{ Concentration $(\mu \mathrm{g} / \mathrm{L})$} & \multirow[b]{2}{*}{ Comment } & \multirow[b]{2}{*}{ Sample } \\
\hline & & & & & & & & $\begin{array}{c}\text { Carbon } \\
\text { Tetrachloride }\end{array}$ & Chloroform & $\begin{array}{l}\text { Methylene } \\
\text { Chloride }\end{array}$ & & \\
\hline \multirow[t]{2}{*}{ Rilinger } & - & $4 / 15 / 08$ & - & - & Pump & - & - & 9.9 & $0.4 \mathrm{~J}$ & ND & - & $\begin{array}{l}\text { MRRILINGER-W- } \\
23243\end{array}$ \\
\hline & & $10 / 21 / 08$ & - & - & Pump & - & - & $0.9 \mathrm{~J}$ & ND & ND & - & $\begin{array}{l}\text { MRRILLINGER-W- } \\
27633\end{array}$ \\
\hline \multirow[t]{8}{*}{ Stone } & $43^{\text {h }}$ & $6 / 4 / 04$ & 23.35 & - & - & - & - & 10 & ND & ND & Purged dry. & $\begin{array}{l}\text { MRPRIVSTON-W- } \\
16475\end{array}$ \\
\hline & & $9 / 14 / 05$ & 17.18 & 40 & - & - & - & 2.6 & $0.3 \mathrm{~J}$ & ND & - & MRPRSTON-W-16511 \\
\hline & & $3 / 19 / 06$ & 17.42 & 40 & 100 & gal & _- & 14 & $0.8 \mathrm{~J}$ & $0.4 \mathrm{~J} \mathrm{~B}$ & - & MRSTONE-W-19987 \\
\hline & & 9/19/06 & 18.55 & 38.8 & 41 & gal & - & 2.1 & ND & ND & - & MRSTONE-W-16529 \\
\hline & & $3 / 22 / 07$ & 20.62 & 38.8 & 56 & gal & - & 5.4 & $0.3 \mathrm{~J}$ & ND & - & MRSTONE-W-16560 \\
\hline & & $10 / 3 / 07$ & 14.6 & 38.6 & 72 & gal & - & 2.8 & ND & ND & - & MRSTONE-W-16589 \\
\hline & & $4 / 15 / 08$ & - & 38.86 & - & - & - & $0.9 \mathrm{~J}$ & ND & ND & - & MRSTONE-W-23244 \\
\hline & & $10 / 21 / 08$ & - & & 5 & gal & - & 3.0 & ND & ND & - & MRSTONE-W-27634 \\
\hline TD12 & $27-67$ & $4 / 22 / 08$ & - & - & - & - & - & ND & ND & ND & $\begin{array}{l}\text { Grimm irrigation } \\
\text { well. }\end{array}$ & MRTD12-W-23264 \\
\hline TD12 & $27-67$ & $10 / 20 / 08$ & - & - & - & - & - & ND & ND & ND & $\begin{array}{l}\text { Grimm irrigation } \\
\text { well. }\end{array}$ & MRTD12-W-27635 \\
\hline
\end{tabular}

a Sampling with the low-flow procedure is described in Section 2.2. Samples not marked with shading were collected after full purging of approximately three well volumes (until field parameters were stable), except as noted differently. The comparison sampling in October 2008 at wells MW1S, MS2S, MW3S, and MW11S was requested by the KDHE (2008).

b Depths were measured from the top of the casing (TOC).

c ND, not detected at instrument detection limit of $0.1 \mu \mathrm{g} / \mathrm{L}$.

d Qualifier J indicates an estimated concentration below the method quantitation limit of $1.0 \mu \mathrm{g} / \mathrm{L}$.

e Qualifier B indicates that the compound was present in the associated method blank.

$f$ Qualifier R indicates that the contaminant was present in the associated equipment rinsate. Resampling confirmed that the well was free of contamination.

g The well's dedicated pump was used for sampling. The pump was allowed to run before the sample was collected.

h Total depth 
TABLE 3.3 Field measurements for groundwater samples collected at Morrill, October 2003 to October 2008. Shading indicates sample collection with the low-flow procedure. ${ }^{\mathrm{a}}$

\begin{tabular}{|c|c|c|c|c|c|c|c|c|c|c|c|c|c|c|c|}
\hline Location & $\begin{array}{l}\text { Screen } \\
\text { Interval } \\
\text { (ft BGL) }\end{array}$ & $\begin{array}{l}\text { Sample } \\
\text { Date }\end{array}$ & $\begin{array}{l}\text { Depth to } \\
\text { Water } \\
\text { (ft TOC) }\end{array}$ & $\begin{array}{l}\text { Depth of } \\
\text { well } \\
\text { (ft TOC) }\end{array}$ & $\begin{array}{l}\text { Volume } \\
\text { Purged }\end{array}$ & $\begin{array}{l}\text { Volume } \\
\text { Units }\end{array}$ & $\begin{array}{l}\text { Pump } \\
\text { Intake } \\
\text { (ft BGL) }\end{array}$ & $\begin{array}{c}\text { Temperature } \\
\left({ }^{\circ} \mathrm{C}\right)\end{array}$ & $\mathrm{pH}$ & $\begin{array}{l}\text { Conductivity } \\
(\mu \mathrm{S} / \mathrm{cm})\end{array}$ & $\begin{array}{c}\text { Dissolved } \\
\text { Oxygen } \\
(\mathrm{mg} / \mathrm{L})\end{array}$ & $\begin{array}{l}\text { ORP } \\
(\mathrm{mV})\end{array}$ & $\begin{array}{l}\text { Iron II } \\
(\mathrm{mg} / \mathrm{L})\end{array}$ & $\begin{array}{l}\text { Carbon } \\
\text { Dioxide } \\
\text { (mg/L) }\end{array}$ & Sample \\
\hline \multirow[t]{14}{*}{ MW1S } & $11-51$ & $10 / 23 / 03$ & 30.36 & 54.04 & 70 & gal & - & 14.6 & 7.14 & 933 & $-{ }^{b}$ & 13 & - & - & MRMW1S-W-16422 \\
\hline & & $6 / 2 / 04$ & 26.97 & 53.94 & 53 & gal & - & 14.4 & 7.16 & 970 & - & - & - & - & MRMW1S-W-16461 \\
\hline & & $9 / 13 / 05$ & 24.16 & 53.9 & 57 & gal & - & 15.3 & 6.95 & 1174 & 7.17 & 200 & 0 & 55 & MRMW1S-W-19259 \\
\hline & & $3 / 22 / 06$ & 29 & 53.95 & 48 & gal & - & 15.5 & 7.23 & 927 & 9.94 & 220 & 0.01 & 40 & MRMW1S-W-20008 \\
\hline & & 9/20/06 & 26.82 & 53.97 & 55 & gal & - & 15.7 & 7.12 & 973 & 7.52 & - & 0.03 & 40 & MRMW1S-W-22495 \\
\hline & & $3 / 21 / 07$ & 25.8 & 53.98 & 55 & gal & - & 16.6 & 6.48 & 960 & 5.45 & 88 & 0 & 40 & MRMW1S-W-16488 \\
\hline & & $10 / 1 / 07$ & 21.65 & 53.95 & 63 & gal & - & 16.0 & 6.80 & 886 & 6.79 & 128 & 0 & 30 & MRMW1S-W-16595 \\
\hline & & $4 / 14 / 08$ & 16.2 & 54 & 5.5 & L & - & 13.9 & 7.09 & 1237 & 6.38 & 118 & 0.02 & - & MRMW1S-W-23230 \\
\hline & & $4 / 22 / 08$ & 16 & 54 & 6.25 & L & - & 15.6 & 6.96 & 1230 & 6.10 & 133 & - & - & MRMW1S-W-23259 \\
\hline & & $5 / 1 / 08$ & - & - & 3.24 & L & 22 & 17.1 & 7.11 & 801 & 3.34 & 104 & - & - & MRMW1S-22-W-23275 \\
\hline & & $5 / 1 / 08$ & - & - & 3.24 & $\mathrm{~L}$ & 27 & 17.0 & 7.10 & 820 & 3.40 & 102 & - & - & MRMW1S-27-W-23276 \\
\hline & & $5 / 1 / 08$ & - & - & 4.25 & L & 48 & 16.3 & 7.00 & 1301 & 3.03 & 118 & - & - & MRMW1S-48-W-23277 \\
\hline & & $10 / 20 / 08$ & 25.8 & 54 & 6 & $\mathrm{~L}$ & 31 & 14.0 & 6.84 & 1265 & 5.40 & 103 & 0 & - & MRMW1S-W-27620 \\
\hline & & $10 / 21 / 08$ & - & - & 57 & gal & & 14.8 & 7.02 & 978 & - & - & 0 & - & MRMW1S-W-27649 \\
\hline \multirow[t]{9}{*}{ MW1D } & $63-88$ & $10 / 22 / 03$ & 28.39 & 88.5 & 92 & gal & - & 14.9 & 6.87 & 2620 & - & 25 & - & _- & MRMW1D-W-16421 \\
\hline & & $6 / 2 / 04$ & 26.82 & 88.6 & 140 & gal & - & 13.9 & 6.87 & 2460 & - & - & - & - & MRMW1D-W-16458 \\
\hline & & $9 / 13 / 05$ & 23.72 & 88.6 & 200 & gal & - & 15.5 & 6.56 & 2470 & - & - & - & - & MRMW1D-W-16518 \\
\hline & & 3/19/06 & 26.85 & 88.6 & 112 & gal & - & 12.9 & 6.95 & 2460 & 5.11 & 230 & 0 & - & MRMW1D-W-19986 \\
\hline & & 9/20/06 & 25.52 & 88.8 & 125 & gal & - & 12.5 & 6.93 & 2690 & - & - & - & - & MRMW1D-W-16532 \\
\hline & & $3 / 21 / 07$ & 25.79 & 88.8 & 125 & gal & - & 15.3 & 6.39 & 2540 & 0.08 & 12 & 0.39 & 40 & MRMW1D-W-16487 \\
\hline & & $10 / 1 / 07$ & 22.75 & 89.38 & 130 & gal & - & 16.3 & 6.60 & 2230 & 6.79 & 5 & 0.44 & 45 & MRMW1D-W-16596 \\
\hline & & $4 / 14 / 08$ & 29.51 & 89 & 6 & $\mathrm{~L}$ & - & 14.6 & 6.99 & 2637 & 0.50 & 32 & 0.73 & - & MRMW1D-W-23231 \\
\hline & & $10 / 20 / 08$ & 30.4 & 89 & 7 & $\mathrm{~L}$ & 75.5 & 13.4 & 6.83 & 2556 & 0.24 & 21 & 0.37 & - & MRMW1D-W-27621 \\
\hline \multirow[t]{10}{*}{ MW2S } & $13-53$ & $10 / 22 / 03$ & 42.21 & 53.35 & $\mathrm{PDS}^{\mathrm{c}}$ & - & - & 16.2 & 6.86 & 875 & - & 20 & - & - & MRMW02-W-16419 \\
\hline & & $6 / 2 / 04$ & 37.44 & 53.3 & 31 & gal & - & 16.9 & 7.07 & 861 & - & - & - & - & MRMW2S-W-16459 \\
\hline & & 9/14/05 & 33.68 & 53.33 & 38 & gal & - & 15.2 & 6.94 & 801 & 7.85 & 142 & - & 65 & MRMW2S-W-19264 \\
\hline & & $3 / 21 / 06$ & 40.87 & 53.32 & 27 & gal & - & 13.0 & 7.07 & 863 & 9.40 & 262 & 0.14 & 25 & MRMW2S-W-19992 \\
\hline & & 9/18/06 & 36.53 & 53.3 & 28 & gal & - & 13.6 & 6.99 & 844 & 6.81 & 69 & 0 & 80 & MRMW2S-W-22488 \\
\hline & & $3 / 22 / 07$ & 35.77 & 53.3 & 35 & gal & - & 15.2 & 6.40 & 790 & 5.82 & 69 & 0 & 30 & MRMW2S-W-16559 \\
\hline & & $10 / 3 / 07$ & 31.17 & 53.4 & 44 & gal & - & 16.8 & 6.97 & 703 & 6.70 & 269 & 0.01 & 30 & MRMW2S-W-16587 \\
\hline & & $4 / 15 / 08$ & 23.55 & 53.4 & 2.22 & $\mathrm{~L}$ & - & 11.7 & 7.23 & 742 & 3.22 & 75 & 0 & - & MRMW2S-W-23232 \\
\hline & & $10 / 21 / 08$ & 33.7 & 53.5 & 5 & $\bar{L}$ & 33 & 13.4 & 7.08 & 745 & 8.55 & 104 & 0 & - & MRMW2S-W-27622 \\
\hline & & $10 / 21 / 08$ & - & - & 39 & gal & - & - & - & - & - & - & - & - & MRMW2S-W-27652 \\
\hline \multirow[t]{12}{*}{ MW3S } & $18-48$ & $10 / 23 / 03$ & 36.47 & 47.79 & 73 & gal & - & 16.8 & 7.23 & 655 & - & 6 & - & - & MRMW03-W-16423 \\
\hline & & $6 / 2 / 04$ & 30.67 & 47.5 & 34 & gal & - & 14.2 & 7.23 & 664 & _- & - & - & - & MRMW3S-W-16462 \\
\hline & & $9 / 13 / 05$ & 25.6 & 47.6 & 50 & gal & - & 14.6 & 7.13 & 663 & 8.82 & 223 & 0 & 100 & MRMW3S-W-19261 \\
\hline & & $3 / 23 / 06$ & 35.62 & 47.74 & 28 & gal & - & $\begin{array}{r}8.9 \\
8.9\end{array}$ & 7.16 & 662 & 6.74 & 269 & 0.08 & 25 & MRMW3S-W-19994 \\
\hline & & 9/20/06 & 29.44 & 47.75 & 22 & gal & - & 12.9 & 7.15 & 669 & 7.64 & 105 & 0 & - & MRMW3S-W-22496 \\
\hline & & $3 / 22 / 07$ & 26.19 & 47.75 & 45 & gal & - & 15.0 & 6.44 & 578 & 5.90 & 261 & 0.17 & 30 & MRMW3S-W-16563 \\
\hline & & $10 / 3 / 07$ & 22.7 & 47.9 & 50 & gal & - & 15.3 & 6.97 & 594 & 0.38 & 282 & 0 & 20 & MRMW3S-W-16585 \\
\hline & & $4 / 14 / 08$ & 16.95 & 47.8 & 3.31 & L & - & 13.7 & 7.17 & 693 & 3.52 & 165 & 0 & - & MRMW3S-W-23233 \\
\hline & & $4 / 22 / 08$ & 15.8 & 47.8 & 6.5 & L & - & 16.0 & 6.99 & 685 & 6.71 & 155 & - & - & MRMW3S-W-23260 \\
\hline & & $5 / 1 / 08$ & - & - & 2.62 & L & 26 & 13.2 & 7.17 & 675 & 3.83 & 161 & - & - & MRMW3S-26-W-23269 \\
\hline & & $5 / 1 / 08$ & - & - & 2.75 & L & 38 & 12.7 & 7.12 & 671 & 4.21 & 193 & - & - & MRMW3S-38-W-23270 \\
\hline & & $5 / 1 / 08$ & - & - & 3.17 & L & 45 & 12.6 & 7.03 & 675 & 4.57 & 205 & - & - & MRMW3S-45-W-23271 \\
\hline
\end{tabular}


TABLE 3.3 (Cont.)

\begin{tabular}{|c|c|c|c|c|c|c|c|c|c|c|c|c|c|c|c|}
\hline Location & $\begin{array}{l}\text { Screen } \\
\text { Interval } \\
\text { (ft BGL) }\end{array}$ & $\begin{array}{l}\text { Sample } \\
\text { Date }\end{array}$ & $\begin{array}{l}\text { Depth to } \\
\text { Water } \\
\text { (ft TOC) }\end{array}$ & $\begin{array}{l}\text { Depth of } \\
\text { well } \\
\text { (ft TOC) }\end{array}$ & $\begin{array}{l}\text { Volume } \\
\text { Purged }\end{array}$ & $\begin{array}{l}\text { Volume } \\
\text { Units }\end{array}$ & $\begin{array}{l}\text { Pump } \\
\text { Intake } \\
\text { (ft BGL) }\end{array}$ & $\begin{array}{c}\text { Temperature } \\
\left({ }^{\circ} \mathrm{C}\right)\end{array}$ & $\mathrm{pH}$ & $\begin{array}{c}\text { Conductivity } \\
(\mu \mathrm{S} / \mathrm{cm})\end{array}$ & $\begin{array}{c}\text { Dissolved } \\
\text { Oxygen } \\
\text { (mg/L) }\end{array}$ & $\begin{array}{l}\text { ORP } \\
(\mathrm{mV})\end{array}$ & $\begin{array}{l}\text { Iron II } \\
\text { (mg/L) }\end{array}$ & $\begin{array}{c}\text { Carbon } \\
\text { Dioxide } \\
(\mathrm{mg} / \mathrm{L})\end{array}$ & Sample \\
\hline \multirow[t]{2}{*}{ MW3S } & $18-48$ & 10/21/08 & 27 & 47.8 & 4.2 & L & 33 & 12.6 & 7.17 & 673 & 6.42 & 115 & 0 & - & MRMW3S-W-27623 \\
\hline & & $10 / 21 / 08$ & - & - & 41 & gal & - & 14.3 & 7.11 & 522 & - & - & 0 & - & MRMW3S-W-27650 \\
\hline \multirow[t]{9}{*}{ MW4S } & $17-47$ & $10 / 21 / 03$ & 46.4 & 47.8 & PDS & - & - & - & 7.17 & 758 & - & - & - & - & MRMW04-W-16418 \\
\hline & & $6 / 4 / 04$ & 43.21 & 47.8 & 10 & gal & - & 15.4 & 6.93 & 769 & - & - & - & - & MRMW4S-W-16470 \\
\hline & & 9/14/05 & 36.21 & 47.81 & 8 & gal & - & 15.4 & 7.30 & 751 & 8.00 & 174 & 0 & 50 & MRMW4S-W-19262 \\
\hline & & $3 / 21 / 06$ & 44.55 & 47.72 & 6 & gal & - & 6.7 & 7.25 & 729 & 10.90 & 154 & 0 & 25 & MRMW4S-W-19993 \\
\hline & & $3 / 22 / 07$ & 38.74 & 47.75 & 6 & gal & - & 14.2 & 6.53 & 765 & 5.91 & 78 & 0.10 & 25 & MRMW4S-W-16562 \\
\hline & & $10 / 3 / 07$ & 31.11 & 47.73 & 30 & gal & - & 16.4 & 6.95 & 715 & 7.40 & 281 & 0.10 & 30 & MRMW4S-W-16586 \\
\hline & & $1 / 11 / 08$ & - & - & - & - & - & 11.3 & 7.56 & 757 & - & - & - & - & MOMW4S-W-011108 \\
\hline & & $4 / 14 / 08$ & 26.32 & 47.85 & 2.48 & $\mathrm{~L}$ & - & 13.1 & 7.28 & 783 & 3.80 & 213 & 0 & - & MRMW4S-W-23234 \\
\hline & & $10 / 20 / 08$ & 36.7 & 47.8 & 8 & gal & - & 14.5 & 7.16 & 756 & 8.71 & 104 & 0 & - & MRMW4S-W-27624 \\
\hline \multirow{11}{*}{ MW5S } & $15-55$ & $10 / 22 / 03$ & 31.4 & 55.72 & 48 & gal & - & 15.3 & 7.10 & 816 & - & 6 & - & - & MRMW05-W-16420 \\
\hline & & $6 / 2 / 04$ & 26.33 & 55.65 & $>57$ & gal & - & 14.3 & 7.21 & 817 & - & - & - & - & MRMW5S-W-16460 \\
\hline & & $9 / 13 / 05$ & 22.66 & 54.2 & 75 & gal & - & 16.0 & 7.04 & 763 & 13.90 & 228 & 0 & 60 & MRMW5S-W-19260 \\
\hline & & $3 / 22 / 06$ & 28.64 & 54.51 & 50 & gal & - & 13.9 & 7.25 & 781 & 4.52 & 234 & 0.06 & 35 & MRMW5S-W-19996 \\
\hline & & 9/20/06 & 25.43 & 54.63 & 52 & gal & - & 13.9 & 7.19 & 787 & 5.82 & 73 & 0 & 35 & MRMW5S-W-22493 \\
\hline & & $4 / 14 / 08$ & 11.2 & 54.6 & 6 & L & - & 14.1 & 6.90 & 1008 & 3.73 & 143 & 0.02 & - & MRMW5S-W-23235 \\
\hline & & $4 / 23 / 08$ & 11.3 & 54.6 & 6.5 & $\bar{L}$ & _- & 14.9 & 6.88 & 1009 & 3.27 & 184 & - & - & MRMW5S-W-23266 \\
\hline & & $5 / 1 / 08$ & - & - & 3.7 & $\bar{L}$ & 20 & 15.2 & 6.92 & 1014 & 2.99 & 126 & - & - & MRMW5S-20-W-23272 \\
\hline & & $5 / 1 / 08$ & - & - & 3.4 & L & 28 & 15.1 & 6.90 & 997 & 2.38 & 124 & - & - & MRMW5S-28-W-23273 \\
\hline & & $5 / 1 / 08$ & - & - & 4 & L & 52 & 15.1 & 6.89 & 989 & 2.06 & 128 & - & - & MRMW5S-52-W-23274 \\
\hline & & $10 / 21 / 08$ & 22.5 & 54.6 & 7 & L & 35 & 13.2 & 7.04 & 818 & 5.54 & 180 & 0 & - & MRMW5S-W-27625 \\
\hline \multirow[t]{8}{*}{ MW6S } & $10-25$ & $6 / 3 / 04$ & 3.34 & 26.9 & 45 & gal & - & 15.1 & 6.89 & 2410 & - & - & - & - & MRMW6S-W-16465 \\
\hline & & 9/14/05 & 4.7 & 26.9 & 43 & gal & - & 14.1 & 7.06 & 2350 & 0.01 & 54 & 0 & 60 & MRMW6S-W-19263 \\
\hline & & $3 / 20 / 06$ & 5.35 & 26.91 & 43 & gal & - & 9.8 & 6.91 & 2360 & 1.37 & 89 & 0.38 & 60 & MRMW6S-W-19990 \\
\hline & & 9/18/06 & 5.48 & 26.92 & 27 & gal & - & 12.5 & 6.96 & 2410 & 0.08 & -29 & 0.35 & 85 & MRMW6S-W-22486 \\
\hline & & $3 / 21 / 07$ & 5.42 & 26.92 & 30 & gal & - & 18.0 & 6.34 & 2450 & 0.12 & 75 & 0.78 & 40 & MRMW6S-W-16486 \\
\hline & & $10 / 2 / 07$ & 5 & 26.9 & 31 & gal & - & 17.1 & 7.33 & 2280 & 0.20 & 61 & 0.19 & 35 & MRMW6S-W-16583 \\
\hline & & $4 / 15 / 08$ & 5.15 & 26.9 & 2.5 & L & - & 8.7 & 6.99 & 2485 & 0.31 & -76 & 0.41 & - & MRMW6S-W-23236 \\
\hline & & 10/20/08 & 5.7 & 26.9 & 5 & L & 17.5 & 14.3 & 6.84 & 2380 & 0.36 & 18 & 0.28 & - & MRMW6S-W-27626 \\
\hline \multirow[t]{8}{*}{ MW7S } & $20-45$ & $6 / 3 / 04$ & 26.68 & 46.98 & 40 & gal & _- & 13.8 & 7.19 & 763 & _- & _- & _- & _- & MRMW7S-W-16466 \\
\hline & & $9 / 12 / 05$ & 17.57 & 46.94 & 55 & gal & - & 15.0 & 7.26 & 760 & 8.35 & 240 & 0 & 50 & MRMW7S-W-19258 \\
\hline & & $3 / 22 / 06$ & 22.45 & 46.98 & 48 & gal & - & 15.2 & 7.32 & 740 & 5.52 & 268 & 0.03 & 25 & MRMW7S-W-20000 \\
\hline & & 9/19/06 & 20.94 & 47.02 & 56 & gal & - & 13.2 & 7.15 & 764 & 7.37 & 114 & 0 & 25 & MRMW7S-W-22490 \\
\hline & & 3/20/07 & 18.01 & 47.02 & 50 & gal & - & 14.6 & 6.43 & 750 & 5.31 & 95 & 0 & 30 & MRMW7S-W-16481 \\
\hline & & $10 / 1 / 07$ & 12.4 & 47 & 70 & gal & - & 15.6 & 6.99 & 725 & 7.76 & 269 & 0.01 & 35 & MRMW7S-W-16581 \\
\hline & & $4 / 14 / 08$ & 7.72 & 47 & 1.82 & L & - & 13.4 & 7.21 & 811 & 2.50 & 276 & 0 & - & MRMW7S-W-23237 \\
\hline & & $4 / 23 / 08$ & 7.8 & 47 & 11 & L & - & 14.0 & 7.00 & 822 & 7.41 & 191 & - & - & MRMW7S-W-23265 \\
\hline
\end{tabular}


TABLE 3.3 (Cont.)

\begin{tabular}{|c|c|c|c|c|c|c|c|c|c|c|c|c|c|c|c|}
\hline Location & $\begin{array}{l}\text { Screen } \\
\text { Interval } \\
\text { (ft BGL) }\end{array}$ & $\begin{array}{l}\text { Sample } \\
\text { Date }\end{array}$ & $\begin{array}{l}\text { Depth to } \\
\text { Water } \\
\text { (ft TOC) }\end{array}$ & $\begin{array}{l}\text { Depth of } \\
\text { well } \\
\text { (ft TOC) }\end{array}$ & $\begin{array}{l}\text { Volume } \\
\text { Purged }\end{array}$ & $\begin{array}{l}\text { Volume } \\
\text { Units }\end{array}$ & $\begin{array}{l}\text { Pump } \\
\text { Intake } \\
\text { (ft BGL) }\end{array}$ & $\begin{array}{c}\text { Temperature } \\
\left({ }^{\circ} \mathrm{C}\right)\end{array}$ & $\mathrm{pH}$ & $\begin{array}{l}\text { Conductivity } \\
(\mu \mathrm{S} / \mathrm{cm})\end{array}$ & $\begin{array}{l}\text { Dissolved } \\
\text { Oxygen } \\
\text { (mg/L) }\end{array}$ & $\begin{array}{l}\text { ORP } \\
(\mathrm{mV})\end{array}$ & $\begin{array}{l}\text { Iron II } \\
(\mathrm{mg} / \mathrm{L})\end{array}$ & $\begin{array}{l}\text { Carbon } \\
\text { Dioxide } \\
\text { (mg/L) }\end{array}$ & Sample \\
\hline \multirow[t]{8}{*}{ MW8S } & $10-25$ & $6 / 3 / 04$ & 3.7 & 26.75 & 45 & gal & - & 12.8 & 7.12 & 941 & - & - & - & - & MRMW8S-W-16464 \\
\hline & & 9/14/05 & 4.02 & 26.82 & 57 & gal & - & 14.1 & 7.30 & 853 & 0.02 & 65 & 0 & 40 & MRMW8S-W-19265 \\
\hline & & 3/20/06 & 4.57 & 26.41 & 43 & gal & - & 12.5 & 7.04 & 954 & 0.90 & 153 & 0.05 & 30 & MRMW8S-W-19991 \\
\hline & & 9/19/06 & 4.76 & 26.83 & 45 & gal & - & 11.8 & 7.09 & 903 & 0.58 & 284 & 0.13 & 50 & MRMW8S-W-22492 \\
\hline & & 3/20/07 & 2.63 & 26.82 & 49 & gal & - & 11.0 & 6.52 & 1026 & 0.77 & 76 & 0 & 30 & MRMW8S-W-16483 \\
\hline & & $10 / 2 / 07$ & 2.2 & 26.8 & 48 & gal & - & 15.2 & 6.76 & 607 & 2.66 & 209 & 0.02 & 25 & MRMW8S-W-16584 \\
\hline & & $4 / 15 / 08$ & 0.7 & 26.8 & 5.5 & $\mathrm{~L}$ & - & 10.2 & 7.27 & 1067 & 1.58 & 170 & 0 & - & MRMW8S-W-23238 \\
\hline & & 10/20/08 & 3.6 & 26.8 & 8 & L & 17.5 & 14.0 & 6.91 & 1002 & 0.93 & 69 & 0 & - & MRMW8S-W-27628 \\
\hline \multirow[t]{6}{*}{ MW9S } & $38.83-53.83$ & 3/22/06 & 20.2 & 58.62 & 20 & gal & _- & 14.6 & 7.17 & 715 & 0.41 & 25 & 0 & 35 & MRMW9S-W-20004 \\
\hline & & 9/19/06 & 18.87 & 59 & 22 & gal & - & 13.0 & 7.08 & 707 & 0.10 & 113 & 0 & 55 & MRMW9S-W-22494 \\
\hline & & 3/20/07 & 16.69 & 59 & 22 & gal & - & 14.2 & 6.39 & 714 & 0.21 & 40 & 0 & 20 & MRMW9S-W-16480 \\
\hline & & $10 / 1 / 07$ & 14 & 58.6 & 23 & gal & - & 15.5 & 7.05 & 664 & 5.50 & 191 & 0 & 30 & MRMW9S-W-16582 \\
\hline & & $4 / 14 / 08$ & 16.58 & 58.63 & 2.29 & $\mathrm{~L}$ & - & 12.6 & 7.33 & 709 & 1.93 & 266 & 0.07 & - & MRMW9S-W-23239 \\
\hline & & $10 / 20 / 08$ & 21.5 & 58.5 & 11 & L & 46.33 & 15.1 & 7.15 & 690 & 6.18 & 106 & 0 & - & MRMW9S-W-27629 \\
\hline \multirow[t]{6}{*}{ MW10S } & $30-45$ & $3 / 21 / 06$ & 12.3 & 49.6 & 19 & gal & - & 6.3 & 7.11 & 701 & 2.10 & 88 & 0.01 & 40 & MRMW10S-W-19999 \\
\hline & & $9 / 18 / 06$ & 11.08 & 49.61 & 20 & gal & - & 14.3 & 7.17 & 701 & 0.04 & 24 & 0.08 & 60 & MRMW10S-W-22489 \\
\hline & & $3 / 21 / 07$ & 10.77 & 49.61 & 20 & gal & - & 14.5 & 6.51 & 720 & 0.88 & 11 & 0 & 30 & MRMW10S-W-16485 \\
\hline & & $10 / 1 / 07$ & 6.95 & 49.65 & 20 & gal & - & 16.3 & 6.97 & 664 & 0.35 & 248 & 0.04 & 35 & MRMW10S-W-16593 \\
\hline & & $4 / 14 / 08$ & 9.82 & 49.7 & 1.9 & $\mathrm{~L}$ & - & 16.0 & 7.25 & 723 & 1.25 & 181 & 0 & - & MRMW10S-W-23240 \\
\hline & & 10/20/08 & 13.7 & 49.7 & 5.4 & L & 37.5 & 14.3 & 7.03 & 710 & 1.02 & 56 & 0 & - & MRMW10S-W-27630 \\
\hline \multirow[t]{8}{*}{ MW11S } & $53-68$ & 3/22/06 & 35.2 & 72.5 & 20 & gal & - & 14.8 & 7.33 & 762 & 9.40 & 237 & 0.06 & 30 & MRMW11S-W-20001 \\
\hline & & 9/19/06 & 36 & 73.14 & 20 & gal & - & 13.0 & 7.24 & 764 & 1.42 & 158 & 0.02 & 30 & MRMW11S-W-22491 \\
\hline & & 3/20/07 & 34.65 & 73.14 & 20 & gal & - & 14.6 & 6.33 & 782 & 3.90 & 76 & 0 & 30 & MRMW11S-W-16479 \\
\hline & & $10 / 1 / 07$ & 31.55 & 73 & 20 & gal & - & 16.4 & 6.49 & 624 & 6.57 & 241 & 0.04 & 35 & MRMW11S-W-16594 \\
\hline & & $4 / 15 / 08$ & 29.9 & 72.7 & 5.5 & L & - & 13.9 & 7.30 & 785 & 6.14 & 152 & 0 & - & MRMW11S-W-23241 \\
\hline & & $4 / 22 / 08$ & 30.2 & 72.7 & 7.2 & $\bar{L}$ & - & 15.1 & 7.25 & 790 & 6.22 & 163 & - & - & MRMW11S-W-23261 \\
\hline & & $10 / 20 / 08$ & 37.1 & 72.7 & 9 & L & 60.5 & 14.3 & 7.16 & 756 & 8.95 & 104 & 0 & - & MRMW11S-W-27631 \\
\hline & & $10 / 21 / 08$ & - & - & 18 & gal & - & 14.8 & 7.19 & 766 & - & - & 0 & - & MRMW11S-W-27651 \\
\hline \multirow[t]{8}{*}{ Isch } & - & 2/19/04 & - & - & Pump & - & - & - & - & - & - & - & - & - & MRJR-W-16502 \\
\hline & & 9/14/05 & - & - & Pump & - & - & 20.4 & 6.73 & 2300 & - & - & - & - & MRPRISCH-W-16513 \\
\hline & & $3 / 23 / 06$ & - & - & 20 & gal & - & 13.0 & 7.23 & 9400 & - & - & - & - & MRISCH-W-19989 \\
\hline & & $9 / 19 / 06$ & - & - & Pump & - & - & - & - & - & - & - & - & - & MRISCH-W-16531 \\
\hline & & $3 / 22 / 07$ & - & - & Pump & - & - & - & - & - & - & - & - & - & MRISCH-W-16564 \\
\hline & & $10 / 3 / 07$ & - & - & Pump & - & - & - & - & - & - & - & - & - & MRISCH-W-16590 \\
\hline & & $4 / 15 / 08$ & - & - & Pump & - & - & 12.6 & 7.33 & 3160 & - & - & 0.28 & - & MRISCH-W-23242 \\
\hline & & 10/21/08 & - & - & Pump & - & - & - & - & - & - & - & - & - & MRISCH-W-27632 \\
\hline \multirow[t]{8}{*}{ Rilinger } & - & $6 / 4 / 04$ & - & - & Pump & - & - & 15.9 & 6.99 & 2450 & - & - & - & - & MRPRIVRIL-W-16471 \\
\hline & & 9/14/05 & - & - & Pump & - & - & - & - & - & - & - & - & - & MRPRILL-W-16512 \\
\hline & & 3/19/06 & - & - & Pump & - & - & 11.9 & 7.05 & 2550 & - & - & - & - & MRRILINGER-W-19988 \\
\hline & & 9/19/06 & - & - & Pump & - & - & - & - & - & - & - & - & - & MRRILI-W-16530 \\
\hline & & $3 / 29 / 07$ & - & - & Pump & - & - & - & - & - & - & - & - & - & MRRILINGER-W-16561 \\
\hline & & $10 / 3 / 07$ & - & - & Pump & - & - & - & - & - & - & - & - & - & MRRILINGER-W-16591 \\
\hline & & $1 / 11 / 08$ & - & - & Pump & - & - & 12.2 & 7.46 & 884 & - & - & - & - & MORIL-W-11108 \\
\hline & & $4 / 15 / 08$ & - & - & Pump & - & - & 12.0 & 7.56 & 868 & - & - & 0 & - & MRRILINGER-W-23243 \\
\hline
\end{tabular}


TABLE 3.3 (Cont.)

\begin{tabular}{|c|c|c|c|c|c|c|c|c|c|c|c|c|c|c|c|}
\hline Location & $\begin{array}{l}\text { Screen } \\
\text { Interval } \\
\text { (ft BGL) }\end{array}$ & $\begin{array}{l}\text { Sample } \\
\text { Date }\end{array}$ & $\begin{array}{l}\text { Depth to } \\
\text { Water } \\
\text { (ft TOC) }\end{array}$ & $\begin{array}{l}\text { Depth of } \\
\text { well } \\
\text { (ft TOC) }\end{array}$ & $\begin{array}{l}\text { Volume } \\
\text { Purged }\end{array}$ & $\begin{array}{l}\text { Volume } \\
\text { Units }\end{array}$ & $\begin{array}{l}\text { Pump } \\
\text { Intake } \\
\text { (ft BGL) }\end{array}$ & $\begin{array}{c}\text { Temperature } \\
\left({ }^{\circ} \mathrm{C}\right)\end{array}$ & $\mathrm{pH}$ & $\begin{array}{c}\text { Conductivity } \\
(\mu \mathrm{S} / \mathrm{cm})\end{array}$ & $\begin{array}{l}\text { Dissolved } \\
\text { Oxygen } \\
\text { (mg/L) }\end{array}$ & $\begin{array}{l}\text { ORP } \\
(\mathrm{mV})\end{array}$ & $\begin{array}{l}\text { Iron II } \\
(\mathrm{mg} / \mathrm{L})\end{array}$ & $\begin{array}{l}\text { Carbon } \\
\text { Dioxide } \\
\text { (mg/L) }\end{array}$ & Sample \\
\hline & & 10/21/08 & - & - & Pump & - & - & - & - & - & - & - & - & - & MRRILLINGER-W-27633 \\
\hline \multirow[t]{8}{*}{ Stone } & $43^{d}$ & $6 / 4 / 04$ & 23.35 & - & PDS & - & - & 17.1 & 7.35 & 682 & - & - & - & - & MRPRIVSTON-W-16475 \\
\hline & & 9/14/05 & 17.18 & 40 & - & - & - & 17.3 & 6.81 & 638 & - & - & - & - & MRPRSTON-W-16511 \\
\hline & & 3/19/06 & 17.42 & 40 & 100 & gal & - & 12.9 & 6.42 & 650 & - & 213 & 0 & - & MRSTONE-W-19987 \\
\hline & & 9/19/06 & 18.55 & 38.8 & 41 & gal & - & 16.7 & 7.12 & 639 & - & - & - & - & MRSTONE-W-16529 \\
\hline & & $3 / 22 / 07$ & 20.62 & 38.8 & 56 & gal & - & 16.7 & 6.58 & 679 & 4.71 & 19 & 0.28 & 35 & MRSTONE-W-16560 \\
\hline & & $10 / 3 / 07$ & 14.6 & 38.6 & 72 & gal & - & 16.1 & 6.97 & 564 & 7.07 & 225 & 0.07 & 25 & MRSTONE-W-16589 \\
\hline & & $4 / 15 / 08$ & - & 38.86 & - & - & - & 11.3 & 7.45 & 557 & - & - & 0 & - & MRSTONE-W-23244 \\
\hline & & 10/21/08 & - & & 5 & gal & - & - & - & - & - & - & - & - & MRSTONE-W-27634 \\
\hline
\end{tabular}

a Sampling with the low-flow procedure is described in Section 2.2. Samples not marked with shading were collected after full purging of approximately three well volumes (until field parameters were stable), except as noted differently. The comparison sampling in October 2008 at wells MW1S, MS2S, MW3S, and MW11S was requested by the KDHE (2008).

b Not measured

c PDS, purged dry and then sampled.

d Total depth. 
TABLE 3.4 Results of analyses at the AGEM Laboratory for volatile organic compounds in surface water and sediment samples collected at Morrill, March 2007 to October 2008. ${ }^{a}$

\begin{tabular}{|c|c|c|c|c|c|c|c|}
\hline \multirow[b]{2}{*}{ Location } & \multirow[b]{2}{*}{ Sample } & \multirow[b]{2}{*}{ Date } & \multirow[b]{2}{*}{ Medium } & \multicolumn{4}{|c|}{ Concentration ( $\mu \mathrm{g} / \mathrm{L}$ in water; $\mu \mathrm{g} / \mathrm{kg}$ in sediment) } \\
\hline & & & & $\begin{array}{c}\text { Carbon } \\
\text { Tetrachloride }\end{array}$ & Chloroform & $\begin{array}{l}\text { Methylene } \\
\text { Chloride }\end{array}$ & $\begin{array}{l}\text { Quantitation } \\
\text { Limit }\end{array}$ \\
\hline SM1 & MRSM1-W-16572 & 3/22/07 & Water & $N D^{b}$ & ND & ND & 1 \\
\hline SM1 & MRSM1-S-16573 & 3/22/07 & Sediment & ND & ND & ND & 10 \\
\hline SM1 & MRSM1-W-16583 & 10/8/07 & Water & ND & ND & ND & 1 \\
\hline SM1 & MRSM1-S-16584 & 10/8/07 & Sediment & ND & ND & ND & 10 \\
\hline SM1 & MRSM1-W-23254 & $4 / 14 / 08$ & Water & ND & ND & ND & 1 \\
\hline SM1 & MRSM1-S-23254 & $4 / 14 / 08$ & Sediment & ND & ND & ND & 10 \\
\hline SM1 & MRSM1-W-27644 & 10/20/08 & Water & ND & ND & ND & 1 \\
\hline SM1 & MRSM1-S-27644 & $10 / 20 / 08$ & Sediment & ND & ND & ND & 10 \\
\hline SM2 & MRSM2-W-16574 & 3/22/07 & Water & ND & ND & ND & 1 \\
\hline SM2 & MRSM2-S-16575 & 3/22/07 & Sediment & ND & ND & ND & 10 \\
\hline SM2 & MRSM2-W-16585 & 10/8/07 & Water & ND & ND & ND & 1 \\
\hline SM2 & MRSM2-S-16586 & $10 / 8 / 07$ & Sediment & ND & ND & ND & 10 \\
\hline SM2 & MRSM2-W-23255 & $4 / 14 / 08$ & Water & ND & ND & ND & 1 \\
\hline SM2 & MRSM2-S-23255 & 4/14/08 & Sediment & ND & ND & ND & 10 \\
\hline SM2 & MRSM2-W-27645 & 10/20/08 & Water & ND & ND & ND & 1 \\
\hline SM2 & MRSM2-S-27645 & $10 / 20 / 08$ & Sediment & ND & ND & ND & 10 \\
\hline SM3 & MRSM3-W-16576 & $3 / 22 / 07$ & Water & ND & ND & ND & 1 \\
\hline SM3 & MRSM3-S-16577 & $3 / 22 / 07$ & Sediment & ND & ND & ND & 10 \\
\hline SM3 & MRSM3-W-16587 & $10 / 8 / 07$ & Water & ND & ND & ND & 1 \\
\hline SM3 & MRSM3-S-16588 & $10 / 8 / 07$ & Sediment & ND & ND & ND & 10 \\
\hline SM3 & MRSM3-W-23256 & $4 / 14 / 08$ & Water & ND & ND & ND & 1 \\
\hline SM3 & MRSM3-S-23256 & $4 / 14 / 08$ & Sediment & ND & ND & ND & 10 \\
\hline SM3 & MRSM3-W-27646 & $10 / 20 / 08$ & Water & ND & ND & ND & 1 \\
\hline SM3 & MRSM3-S-27646 & $10 / 20 / 08$ & Sediment & ND & ND & ND & 10 \\
\hline SM4 & MRSM4-W-16578 & $3 / 22 / 07$ & Water & ND & ND & ND & 1 \\
\hline SM4 & MRSM4-S-16579 & $3 / 22 / 07$ & Sediment & ND & ND & ND & 10 \\
\hline SM4 & MRSM4-W-16589 & $10 / 8 / 07$ & Water & ND & ND & ND & 1 \\
\hline SM4 & MRSM4-S-16590 & $10 / 8 / 07$ & Sediment & ND & ND & ND & 10 \\
\hline SM4 & MRSM4-W-23257 & $4 / 14 / 08$ & Water & ND & ND & ND & 1 \\
\hline SM4 & MRSM4-S-23257 & $4 / 14 / 08$ & Sediment & ND & ND & ND & 10 \\
\hline SM4 & MRSM4-W-27647 & 10/20/08 & Water & ND & ND & ND & 1 \\
\hline SM4 & MRSM4-S-27647 & $10 / 20 / 08$ & Sediment & ND & ND & ND & 10 \\
\hline SMB & MRSMB-W-16570 & 3/22/07 & Water & ND & ND & ND & 1 \\
\hline SMB & MRSMB-S-16571 & $3 / 22 / 07$ & Sediment & ND & ND & ND & 10 \\
\hline SMB & MRSMB-W-16581 & $10 / 8 / 07$ & Water & ND & ND & ND & 1 \\
\hline SMB & MRSMB-S-16582 & $10 / 8 / 07$ & Sediment & ND & ND & ND & 10 \\
\hline SMB & MRSMB-W-23258 & $4 / 14 / 08$ & Water & ND & ND & ND & 1 \\
\hline SMB & MRSMB-S-23258 & $4 / 14 / 08$ & Sediment & ND & ND & ND & 10 \\
\hline SMB & MRSMB-W-27648 & $10 / 20 / 08$ & Water & ND & ND & ND & 1 \\
\hline SMB & MRSMB-S-27648 & 10/20/08 & Sediment & ND & ND & ND & 10 \\
\hline
\end{tabular}

a Analyses conducted at the AGEM Laboratory, Argonne, Illinois, with EPA Method 524.2 for surface water samples or modified EPA Method 5030B/8260B for sediment samples.

b ND, not detected at the instrument detection limit of $0.1 \mu \mathrm{g} / \mathrm{L}$ for surface water samples or $1.0 \mu \mathrm{g} / \mathrm{kg}$ for sediment samples. 
TABLE 3.5 Results of analyses at the AGEM Laboratory for carbon tetrachloride and chloroform in vegetation samples collected at Morrill, October 2006 to July $2008 .^{a}$

\begin{tabular}{|c|c|c|c|c|c|}
\hline \multirow[b]{2}{*}{ Location } & \multirow[b]{2}{*}{ Sample } & \multirow[b]{2}{*}{ Date } & \multirow[b]{2}{*}{ Type } & \multicolumn{2}{|c|}{ Concentration $(\mu \mathrm{g} / \mathrm{kg})$} \\
\hline & & & & $\begin{array}{c}\text { Carbon } \\
\text { Tetrachloride }\end{array}$ & Chloroform \\
\hline \multirow[t]{6}{*}{ MR001 } & MR001-B-18959 & $10 / 14 / 06$ & Branch & ND & 1.6 \\
\hline & MR001-L-18958 & $10 / 14 / 06$ & Leaf & ND & 3.3 \\
\hline & MR001-B-23173 & $4 / 2 / 07$ & Branch & ND & ND \\
\hline & MR001-B-23213 & $7 / 26 / 07$ & Branch & ND & ND \\
\hline & MR001-L-23212 & $7 / 26 / 07$ & Leaf & ND & ND \\
\hline & MR001-B-16623 & $7 / 24 / 08$ & Branch & ND & ND \\
\hline MR001A & MR001A-B-16622 & $7 / 24 / 08$ & Branch & ND & ND \\
\hline \multirow[t]{6}{*}{ MR002 } & MR002-B-18961 & $10 / 14 / 06$ & Branch & ND & ND \\
\hline & MR002-L-18960 & $10 / 14 / 06$ & Leaf & ND & ND \\
\hline & MR002-B-23174 & $4 / 2 / 07$ & Branch & ND & 1.5 \\
\hline & MR002-B-23211 & $7 / 26 / 07$ & Branch & ND & ND \\
\hline & MR002-L-23210 & $7 / 26 / 07$ & Leaf & ND & 1.3 \\
\hline & MR002-B-16621 & $7 / 24 / 08$ & Branch & ND & ND \\
\hline \multirow[t]{6}{*}{ MR003 } & MR003-B-18963 & $10 / 14 / 06$ & Branch & ND & 1.5 \\
\hline & MR003-L-18962 & $10 / 14 / 06$ & Leaf & ND & 1.8 \\
\hline & MR003-B-23175 & $4 / 2 / 07$ & Branch & ND & ND \\
\hline & MR003-B-23209 & $7 / 26 / 07$ & Branch & ND & ND \\
\hline & MR003-L-23208 & $7 / 26 / 07$ & Leaf & ND & 0.8 \\
\hline & MR003-B-16620 & $7 / 24 / 08$ & Branch & ND & ND \\
\hline \multirow[t]{6}{*}{ MR004 } & MR004-B-18965 & $10 / 14 / 06$ & Branch & ND & 2.1 \\
\hline & MR004-L-18964 & $10 / 14 / 06$ & Leaf & ND & ND \\
\hline & MR004-B-23176 & $4 / 2 / 07$ & Branch & ND & ND \\
\hline & MR004-B-23205 & $7 / 26 / 07$ & Branch & ND & ND \\
\hline & MR004-L-23204 & $7 / 26 / 07$ & Leaf & ND & ND \\
\hline & MR004-B-16619 & $7 / 24 / 08$ & Branch & ND & ND \\
\hline \multirow[t]{5}{*}{ MR005 } & MR005-B-18967 & $10 / 14 / 06$ & Branch & ND & ND \\
\hline & MR005-L-18966 & $10 / 14 / 06$ & Leaf & ND & 1.2 \\
\hline & MR005-B-23177 & $4 / 2 / 07$ & Branch & ND & ND \\
\hline & MR005-B-23207 & $7 / 26 / 07$ & Branch & ND & ND \\
\hline & MR005-L-23206 & $7 / 26 / 07$ & Leaf & ND & 1.4 \\
\hline MR005A & MR005-B-16618 & $7 / 24 / 08$ & Branch & ND & ND \\
\hline
\end{tabular}


TABLE 3.5 (Cont.)

\begin{tabular}{|c|c|c|c|c|c|}
\hline \multirow[b]{2}{*}{ Location } & \multirow[b]{2}{*}{ Sample } & \multirow[b]{2}{*}{ Date } & \multirow[b]{2}{*}{ Type } & \multicolumn{2}{|c|}{ Concentration $(\mu \mathrm{g} / \mathrm{kg})$} \\
\hline & & & & $\begin{array}{c}\text { Carbon } \\
\text { Tetrachloride }\end{array}$ & Chloroform \\
\hline \multirow[t]{6}{*}{ MR006 } & MR006-B-18969 & $10 / 14 / 06$ & Branch & ND & 3.8 \\
\hline & MR006-L-18968 & $10 / 14 / 06$ & Leaf & ND & ND \\
\hline & MR006-B-23161 & $4 / 2 / 07$ & Branch & ND & ND \\
\hline & MR006-B-23181 & $7 / 26 / 07$ & Branch & ND & ND \\
\hline & MR006-L-23180 & $7 / 26 / 07$ & Leaf & ND & 1.3 \\
\hline & MR006-B-16625 & $7 / 24 / 08$ & Branch & ND & ND \\
\hline \multirow[t]{6}{*}{ MR007 } & MR007-B-18971 & $10 / 14 / 06$ & Branch & ND & 1.6 \\
\hline & MR007-L-18970 & $10 / 14 / 06$ & Leaf & ND & 2.0 \\
\hline & MR007-B-23162 & $4 / 2 / 07$ & Branch & ND & ND \\
\hline & MR007-B-23183 & $7 / 26 / 07$ & Branch & 0.1 & ND \\
\hline & MR007-L-23182 & $7 / 26 / 07$ & Leaf & ND & 1.3 \\
\hline & MR007-B-16626 & $7 / 24 / 08$ & Branch & ND & ND \\
\hline \multirow[t]{6}{*}{ MR008 } & MR008-B-18973 & $10 / 14 / 06$ & Branch & ND & 1.3 \\
\hline & MR008-L-18972 & $10 / 14 / 06$ & Leaf & ND & 2.5 \\
\hline & MR008-B-23163 & $4 / 2 / 07$ & Branch & ND & 0.8 \\
\hline & MR008-B-23185 & $7 / 26 / 07$ & Branch & ND & ND \\
\hline & MR008-L-23184 & $7 / 26 / 07$ & Leaf & ND & ND \\
\hline & MR008-B-16627 & $7 / 24 / 08$ & Branch & ND & ND \\
\hline \multirow[t]{6}{*}{ MR009 } & MR009-B-18975 & $10 / 14 / 06$ & Branch & ND & 0.9 \\
\hline & MR009-L-18974 & $10 / 14 / 06$ & Leaf & ND & 2.5 \\
\hline & MR009-B-23165 & $4 / 2 / 07$ & Branch & ND & ND \\
\hline & MR009-B-23189 & $7 / 26 / 07$ & Branch & ND & ND \\
\hline & MR009-L-23188 & $7 / 26 / 07$ & Leaf & ND & 1.5 \\
\hline & MR009-B-16629 & $7 / 24 / 08$ & Branch & ND & ND \\
\hline \multirow[t]{6}{*}{ MR010 } & MR010-B-18977 & $10 / 14 / 06$ & Branch & ND & 3.4 \\
\hline & MR010-L-18976 & $10 / 14 / 06$ & Leaf & ND & ND \\
\hline & MR010-B-23167 & $4 / 2 / 07$ & Branch & ND & ND \\
\hline & MR010-B-23193 & $7 / 26 / 07$ & Branch & ND & 0.8 \\
\hline & MR010-L-23192 & $7 / 26 / 07$ & Leaf & ND & 1.4 \\
\hline & MR010-B-16631 & $7 / 24 / 08$ & Branch & 0.14 & 1.4 \\
\hline \multirow[t]{6}{*}{ MR011 } & MR011-B-18979 & $10 / 14 / 06$ & Branch & ND & 2.2 \\
\hline & MR011-L-18978 & $10 / 14 / 06$ & Leaf & ND & 2.6 \\
\hline & MR011-B-23168 & $4 / 2 / 07$ & Branch & ND & ND \\
\hline & MR011-B-23195 & $7 / 26 / 07$ & Branch & ND & ND \\
\hline & MR011-L-23194 & $7 / 26 / 07$ & Leaf & ND & 1.7 \\
\hline & MR011-B-16632 & $7 / 24 / 08$ & Branch & ND & ND \\
\hline
\end{tabular}


TABLE 3.5 (Cont.)

\begin{tabular}{|c|c|c|c|c|c|}
\hline \multirow[b]{2}{*}{ Location } & \multirow[b]{2}{*}{ Sample } & \multirow[b]{2}{*}{ Date } & \multirow[b]{2}{*}{ Type } & \multicolumn{2}{|c|}{ Concentration $(\mu \mathrm{g} / \mathrm{kg})$} \\
\hline & & & & $\begin{array}{c}\text { Carbon } \\
\text { Tetrachloride }\end{array}$ & Chloroform \\
\hline \multirow[t]{6}{*}{ MR012 } & MR012-B-18981 & $10 / 14 / 06$ & Branch & ND & 2.1 \\
\hline & MR012-L-18980 & $10 / 14 / 06$ & Leaf & ND & 2.9 \\
\hline & MR012-B-23169 & $4 / 2 / 07$ & Branch & ND & ND \\
\hline & MR012-B-23197 & $7 / 26 / 07$ & Branch & ND & ND \\
\hline & MR012-L-23196 & $7 / 26 / 07$ & Leaf & ND & 1.4 \\
\hline & MR012-B-16633 & $7 / 24 / 08$ & Branch & ND & ND \\
\hline \multirow[t]{6}{*}{ MR013 } & MR013-B-18983 & $10 / 14 / 06$ & Branch & ND & ND \\
\hline & MR013-L-18982 & $10 / 14 / 06$ & Leaf & ND & 2.2 \\
\hline & MR013-B-23160 & $4 / 2 / 07$ & Branch & ND & ND \\
\hline & MR013-B-23179 & $7 / 26 / 07$ & Branch & ND & ND \\
\hline & MR013-L-23178 & $7 / 26 / 07$ & Leaf & ND & ND \\
\hline & MR013-B-16624 & $7 / 24 / 08$ & Branch & ND & ND \\
\hline \multirow[t]{4}{*}{ MR014 } & MR014-B-23164 & $4 / 2 / 07$ & Branch & ND & ND \\
\hline & MR014-B-23187 & $7 / 26 / 07$ & Branch & 0.28 & ND \\
\hline & MR014-L-23186 & $7 / 26 / 07$ & Leaf & ND & ND \\
\hline & MR014-B-16628 & $7 / 24 / 08$ & Branch & ND & ND \\
\hline \multirow[t]{4}{*}{ MR015 } & MR015-B-23166 & $4 / 2 / 07$ & Branch & ND & 0.8 \\
\hline & MR015-B-23191 & $7 / 26 / 07$ & Branch & ND & ND \\
\hline & MR015-L-23190 & $7 / 26 / 07$ & Leaf & ND & ND \\
\hline & MR015-B-16630 & $7 / 24 / 08$ & Branch & ND & ND \\
\hline \multirow[t]{4}{*}{ MR016 } & MR016-B-23170 & $4 / 2 / 07$ & Branch & ND & 1.1 \\
\hline & MR016-B-23199 & $7 / 26 / 07$ & Branch & ND & ND \\
\hline & MR016-L-23198 & $7 / 26 / 07$ & Leaf & ND & ND \\
\hline & MR016-B-16634 & $7 / 24 / 08$ & Branch & ND & ND \\
\hline \multirow[t]{4}{*}{ MR017 } & MR017-B-23171 & $4 / 2 / 07$ & Branch & ND & ND \\
\hline & MR017-B-23203 & $7 / 26 / 07$ & Branch & ND & ND \\
\hline & MR017-L-23202 & $7 / 26 / 07$ & Leaf & ND & ND \\
\hline & MR017-B-16635 & $7 / 24 / 08$ & Branch & ND & ND \\
\hline \multirow[t]{4}{*}{ MR018 } & MR018-B-23172 & $4 / 2 / 07$ & Branch & ND & ND \\
\hline & MR018-B-23201 & $7 / 26 / 07$ & Branch & ND & ND \\
\hline & MR018-L-23200 & $7 / 26 / 07$ & Leaf & ND & 3.2 \\
\hline & MR018-B-16636 & $7 / 24 / 08$ & Branch & ND & ND \\
\hline MR019 & MR019-B-16637 & $7 / 24 / 08$ & Branch & 0.11 & ND \\
\hline
\end{tabular}


TABLE 3.5 (Cont.)

\begin{tabular}{lccccc}
\hline & & & & \multicolumn{2}{c}{ Concentration $(\mu \mathrm{g} / \mathrm{kg})$} \\
\cline { 3 - 6 } Location & Sample & Date & Type & $\begin{array}{c}\text { Carbon } \\
\text { Tetrachloride }\end{array}$ & Chloroform \\
\hline MR020 & MR020-B-20021 & $7 / 24 / 08$ & Branch & ND & ND \\
MR021 & MR021-B-20022 & $7 / 24 / 08$ & Branch & ND & ND \\
MR022 & MR022-B-20023 & $7 / 24 / 08$ & Branch & ND & ND \\
MR023 & MR023-B-20024 & $7 / 24 / 08$ & Branch & ND & ND \\
MR024 & MR024-B-20025 & $7 / 24 / 08$ & Branch & ND & ND \\
\hline
\end{tabular}

a Analyses conducted at the AGEM Laboratory, Argonne, Illinois, with modified EPA Method 5021 (headspace analysis on a gas chromatograph with electron capture detection).

b ND, not detected at a method reporting limit of $0.1 \mu \mathrm{g} / \mathrm{kg}$ for carbon tetrachloride or $0.75 \mu \mathrm{g} / \mathrm{kg}$ for chloroform. 
TABLE 3.6 Results from the AGEM Laboratory and TestAmerica (TA) for sampling of selected wells with low-flow purging method or purging of three well volumes. Shading indicates sample collection with the low-flow procedure. ${ }^{\text {a }}$

Carbon Tetrachloride $(\mu \mathrm{g} / \mathrm{L})$

\begin{tabular}{|c|c|c|c|c|c|c|c|}
\hline Well & $\begin{array}{l}\text { Low Flow } \\
\text { AGEM }\end{array}$ & $\begin{array}{c}\text { Full Purge } \\
\text { AGEM }\end{array}$ & $\begin{array}{c}\text { Low Flow } \\
\text { TA }\end{array}$ & $\begin{array}{c}\text { Full Purge } \\
\text { TA }\end{array}$ & $\begin{array}{c}\text { Purging } \\
\text { Method } \\
\text { Difference } \\
\text { AGEM }\end{array}$ & $\begin{array}{c}\text { Low Flow } \\
\text { Lab Difference } \\
\text { AGEM vs. TA }\end{array}$ & $\begin{array}{c}\text { Full Purge } \\
\text { Lab Difference } \\
\text { AGEM vs. TA }\end{array}$ \\
\hline MW1S & $0.7 \mathrm{~J}^{\mathrm{c}}$ & 35 & 0.64 & 29 & 34.3 & 0.06 & 6 \\
\hline MW2S & $N D^{d}$ & ND & ND & ND & - & - & - \\
\hline MW3S & 55 & 63 & 49 & 54 & 8 & 6 & 9 \\
\hline MW11S & 42 & 45 & 35 & 27 & 3 & 7 & 18 \\
\hline & \multicolumn{7}{|c|}{ Chloroform $(\mu \mathrm{g} / \mathrm{L})$} \\
\hline Well & $\begin{array}{c}\text { Low Flow } \\
\text { AGEM }\end{array}$ & $\begin{array}{l}\text { Full Purge } \\
\text { AGEM }\end{array}$ & $\begin{array}{c}\text { Low Flow } \\
\text { TA }\end{array}$ & $\begin{array}{c}\text { Full Purge } \\
\text { TA }\end{array}$ & $\begin{array}{c}\text { Purging } \\
\text { Method } \\
\text { Difference } \\
\text { AGEM }\end{array}$ & $\begin{array}{c}\text { Low Flow } \\
\text { Lab Difference } \\
\text { AGEM vs. TA }\end{array}$ & $\begin{array}{c}\text { Full Purge } \\
\text { Lab Difference } \\
\text { AGEM vs. TA }\end{array}$ \\
\hline MW1S & ND & 1.8 & ND & 1.7 & 1.8 & - & 0.1 \\
\hline MW2S & ND & ND & ND & ND & - & - & - \\
\hline MW3S & 1.4 & 1.6 & 1.5 & 1.6 & 0.2 & 0.1 & 0 \\
\hline MW11S & $0.9 \mathrm{~J}$ & $0.9 \mathrm{~J}$ & 0.93 & 0.79 & 0 & 0.03 & 0.11 \\
\hline
\end{tabular}

a Sampling with the low-flow procedure is described in Section 2.2. Samples not marked with shading were collected after full purging of approximately three well volumes (until field parameters were stable), except as noted differently. The comparison sampling in October 2008 at wells MW1S, MS2S, MW3S, and MW11S was requested by the KDHE (2008).

b In the low-flow sampling of well MW1S in October 2008, the pump intake was positioned too near the static water level (which had declined since April 2008). The low contaminant concentrations in the samples collected with the low-flow method are considered non-representative of site conditions.

c Qualifier J indicates an estimated concentration below the method quantitation limit of $1.0 \mu \mathrm{g} / \mathrm{L}$.

${ }^{d} \mathrm{ND}$, not detected at instrument detection limit of $0.1 \mu \mathrm{g} / \mathrm{L}$. 


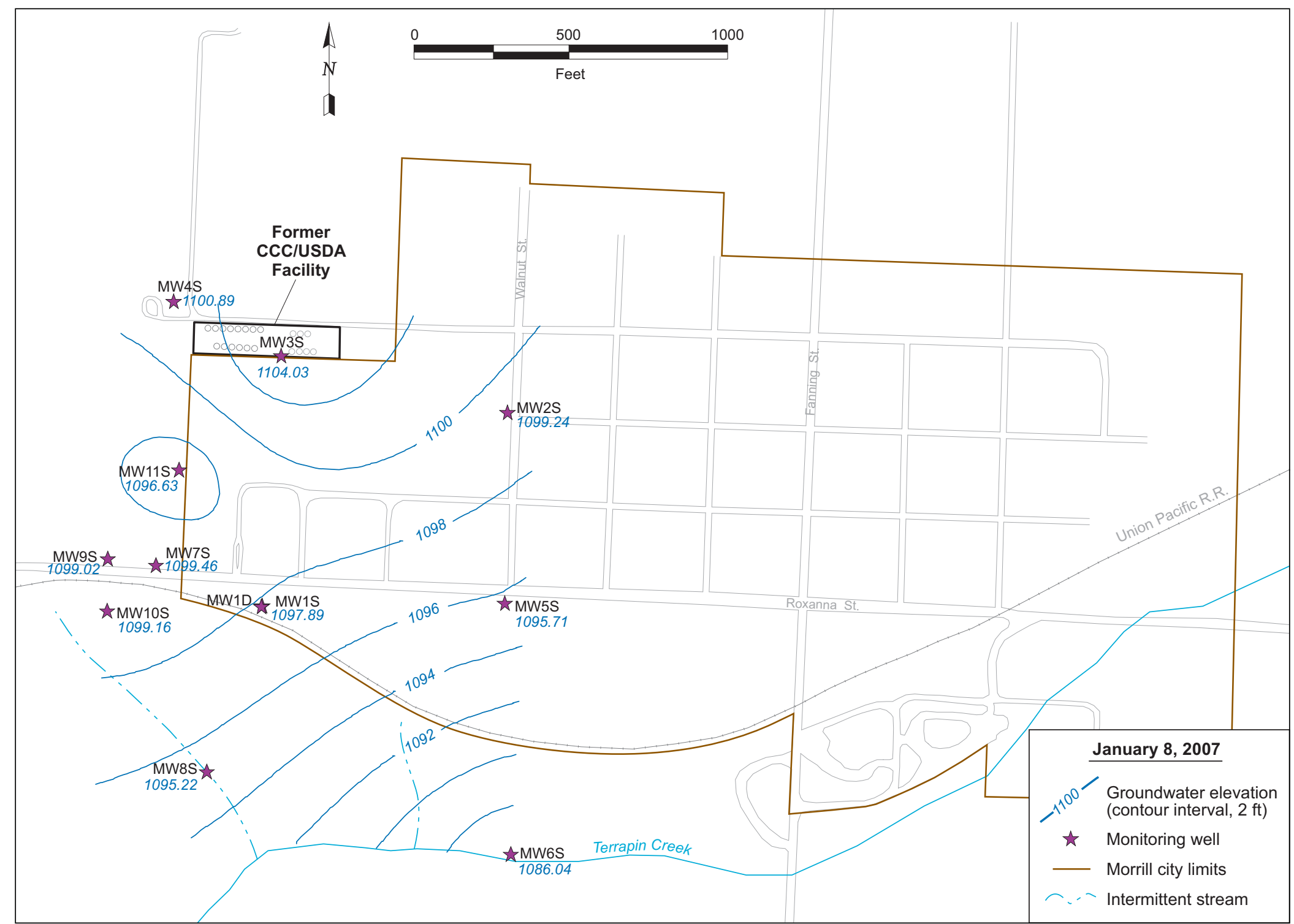

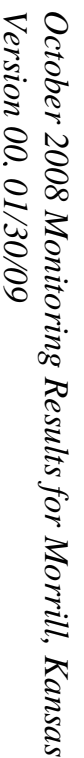

FIGURE 3.1a Potentiometric surface at Morrill, based on water levels measured manually on January 8, 2007. 


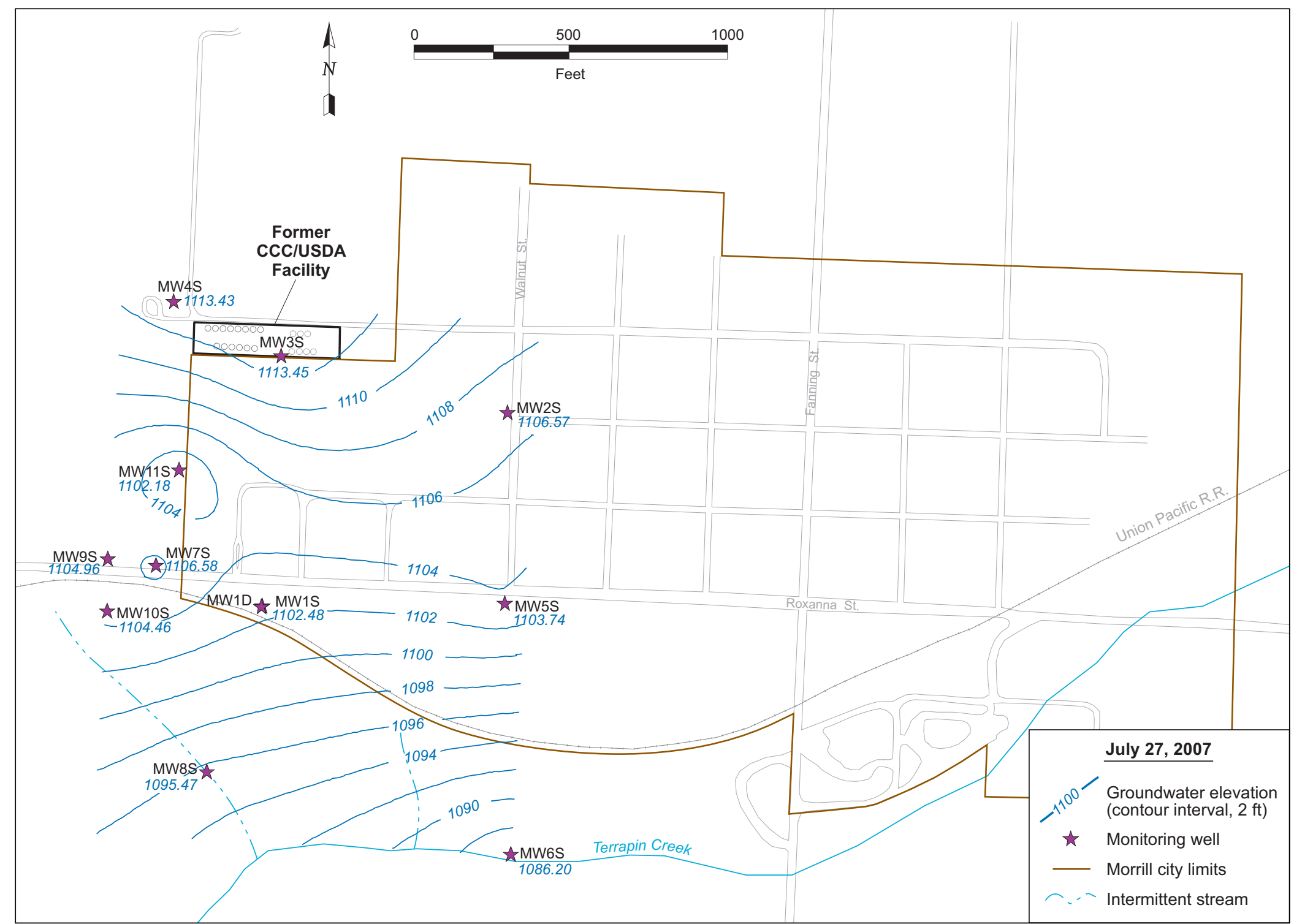

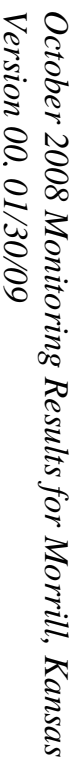

FIGURE 3.1b Potentiometric surface at Morrill, based on water levels measured manually on July 27, 2007. 


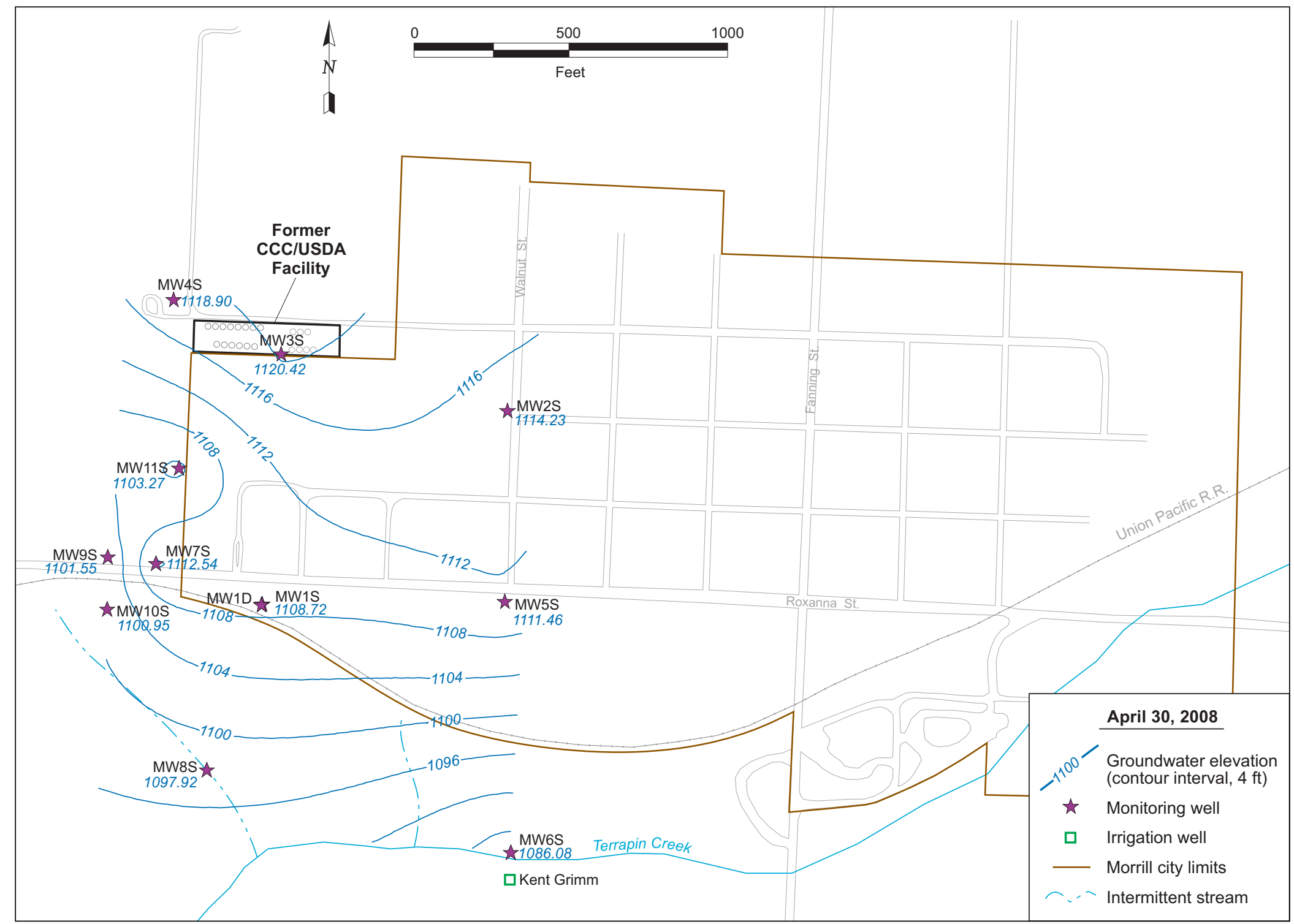

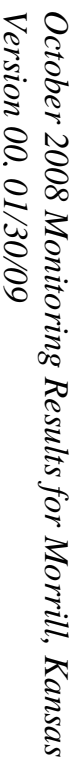

FIGURE 3.1c Potentiometric surface at Morrill, based on water levels measured manually on April 30, 2008. 


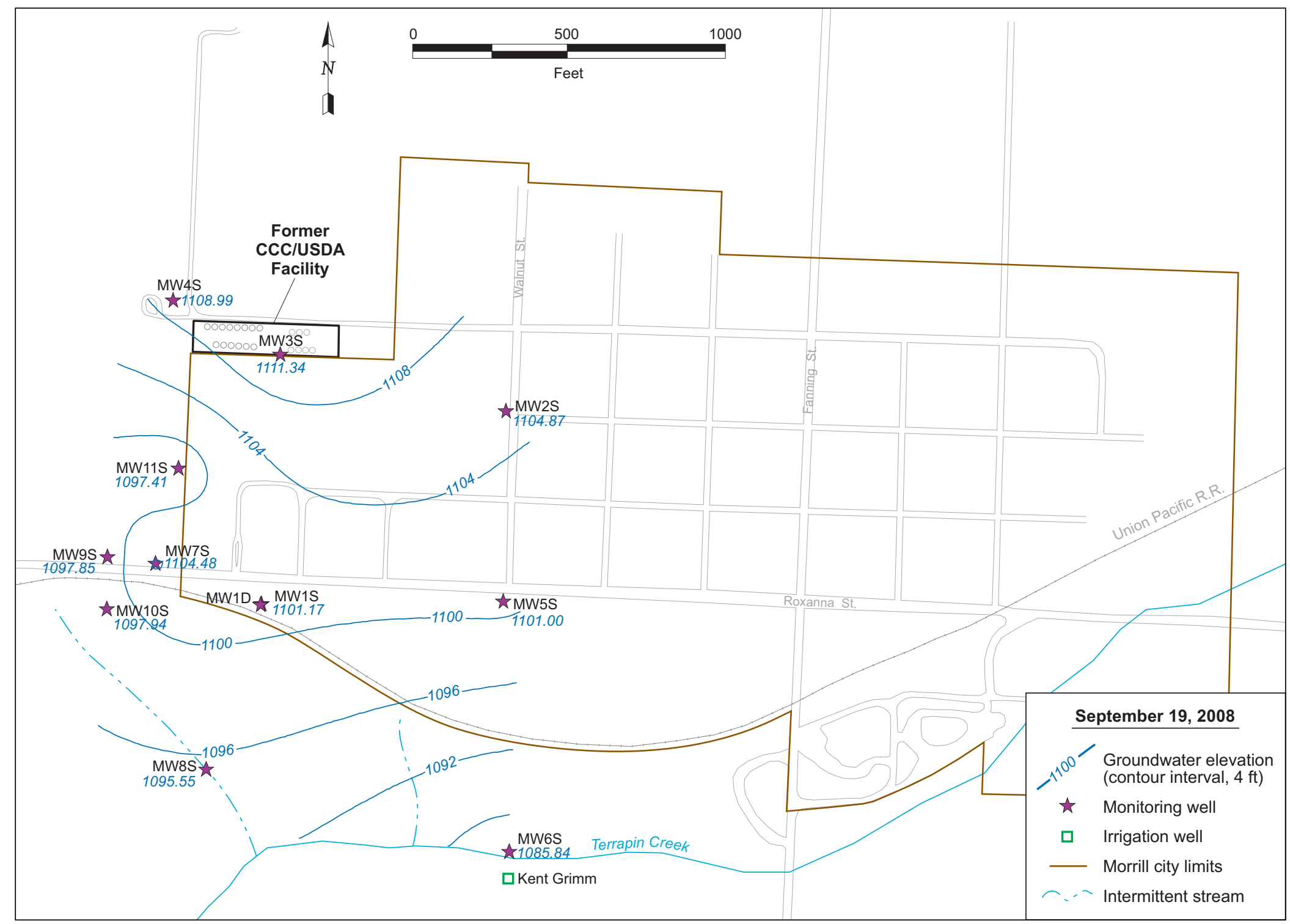

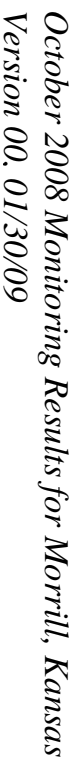

FIGURE 3.1d Potentiometric surface at Morrill, based on water levels measured manually on September 19, 2008. 
Morrill Water Levels

1-1-07 to 12-31-07

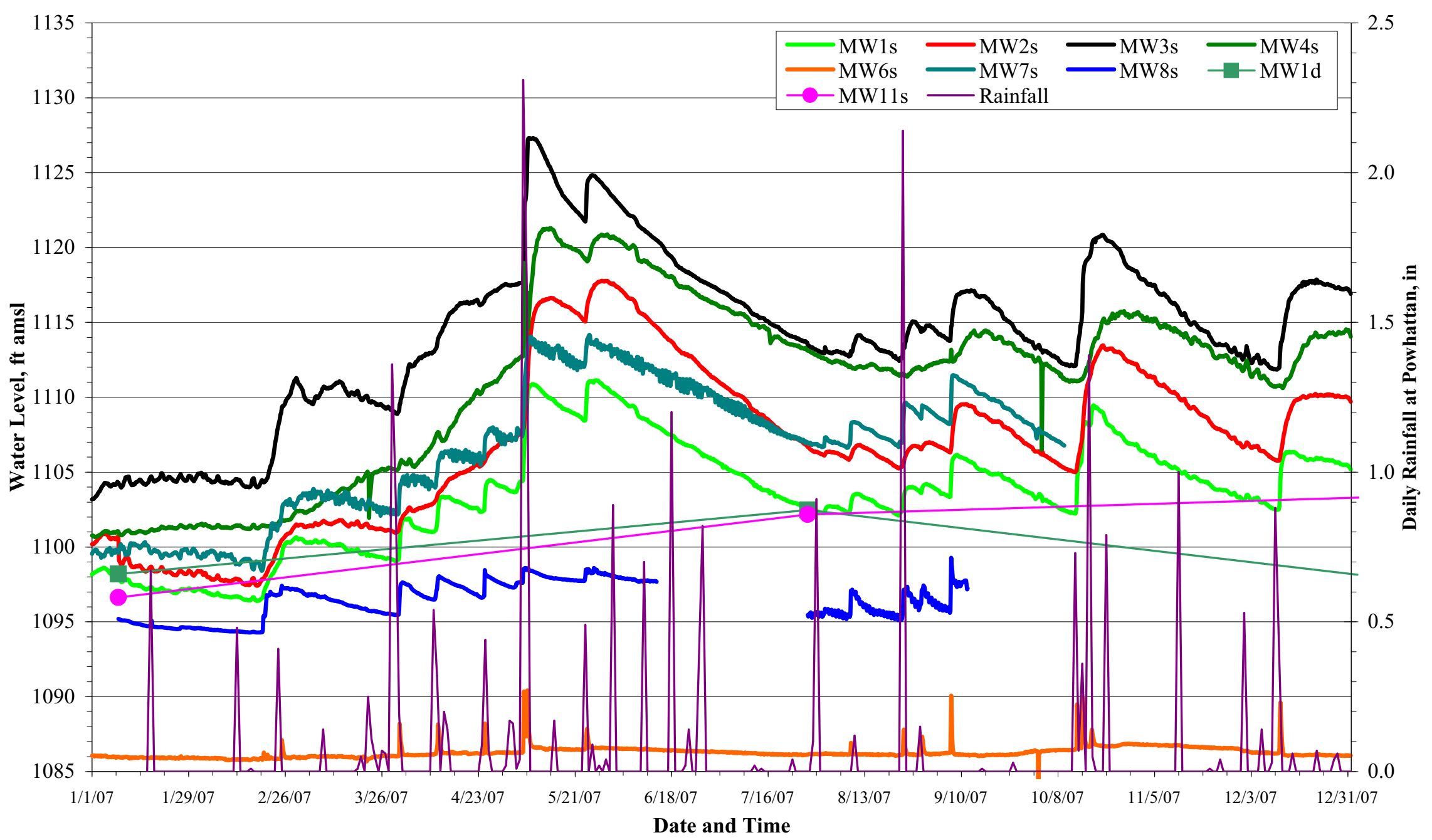

FIGURE 3.2a Hydrographs summarizing results of long-term water level monitoring in wells MW1S-MW4S and MW6S-MW8S at Morrill, from January 1, 2007, to December 31, 2007. 
Morrill Water Levels

1-1-08 to 9-19-08

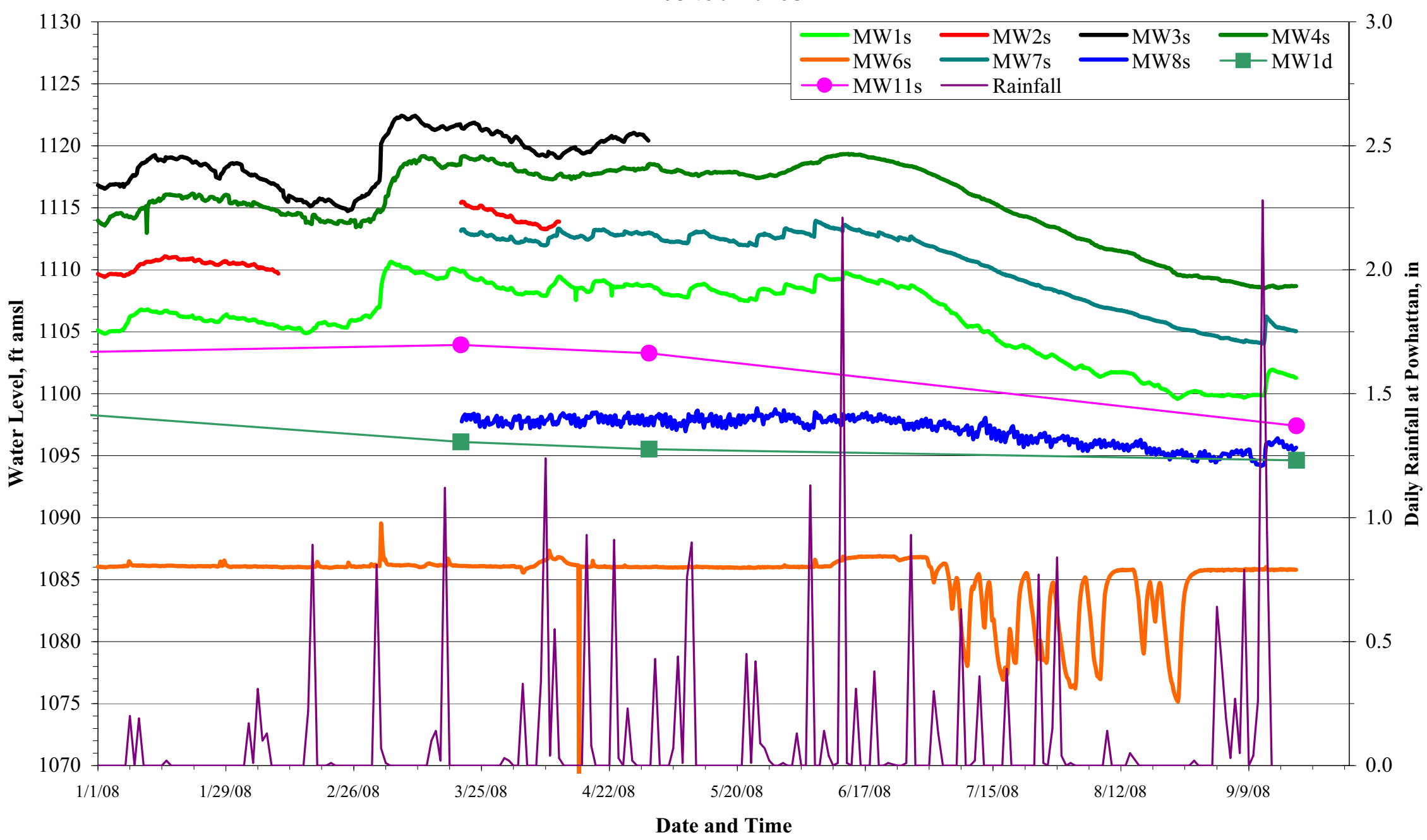

FIGURE 3.2b Hydrographs summarizing results of long-term water level monitoring in wells MW1S-MW4S and MW6S-MW8S at Morrill, from January 1, 2008, to September 19, 2008. 


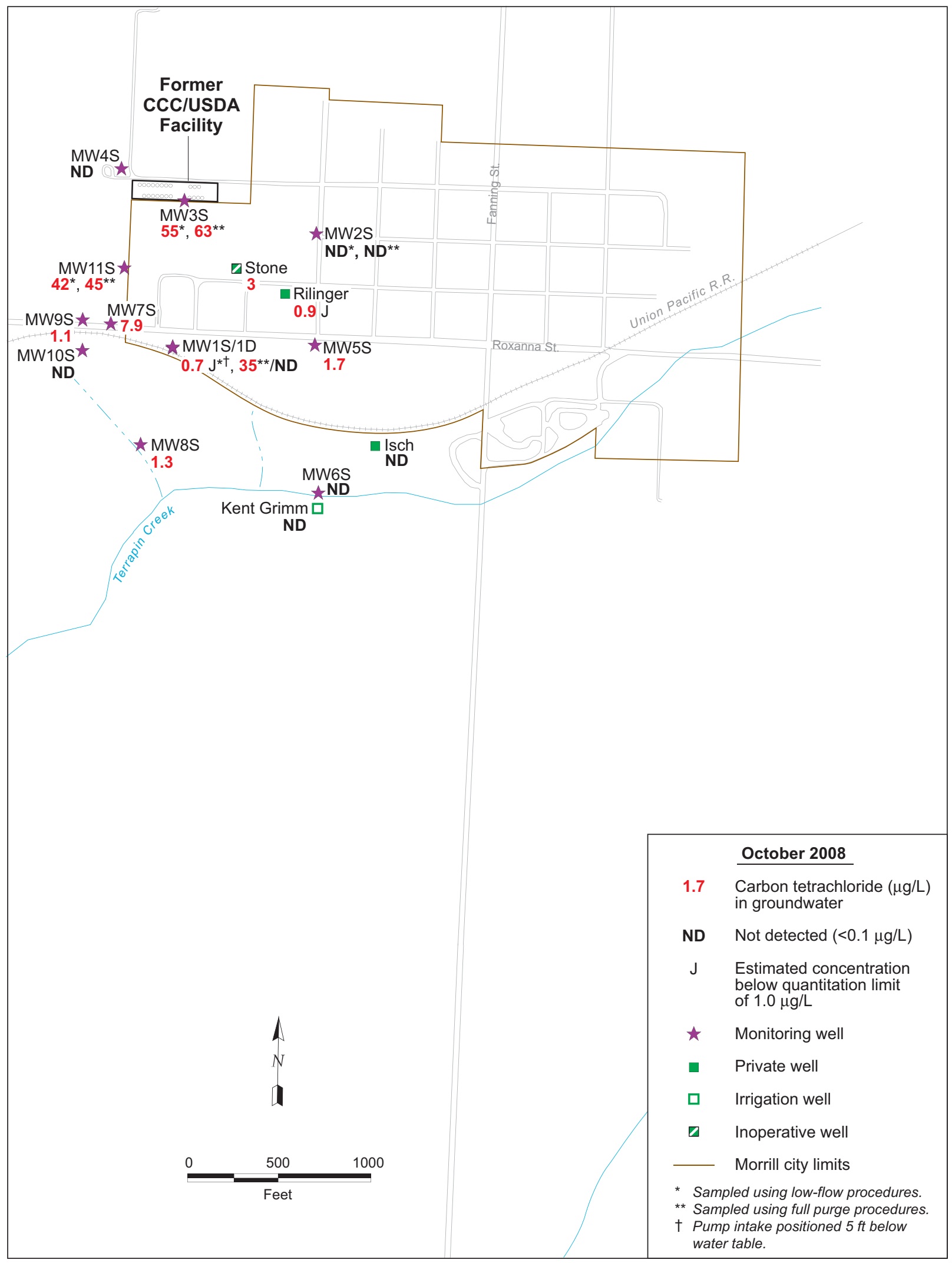

FIGURE 3.3 Carbon tetrachloride concentrations in groundwater at Morrill, October 2008. 

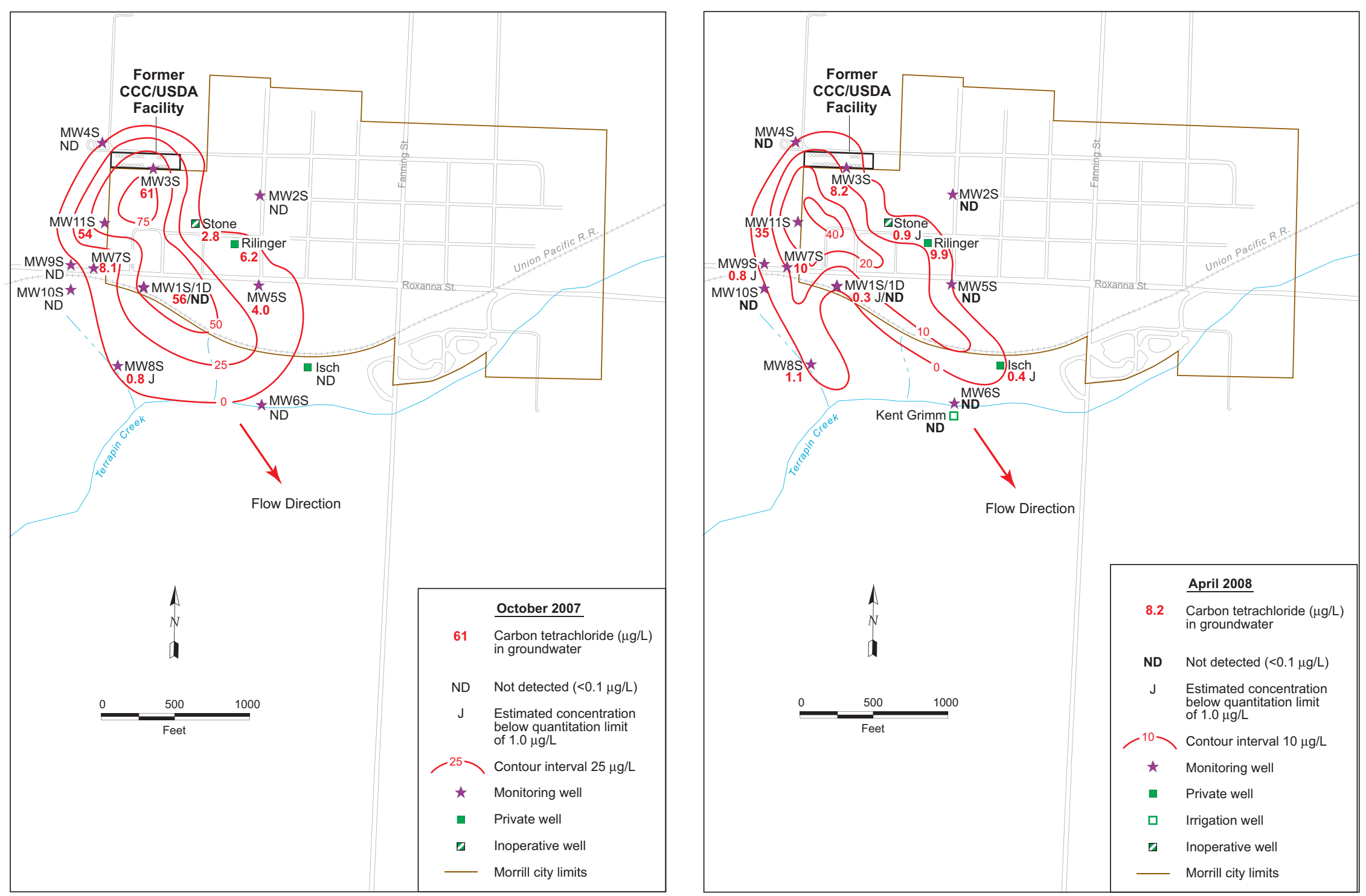

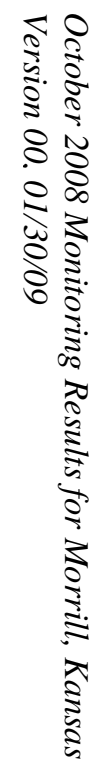

FIGURE 3.4 Lateral extent of the carbon tetrachloride contamination in groundwater at Morrill, as interpreted on the basis of sampling and analysis in October 2007 (left) and April 2008 (right). 


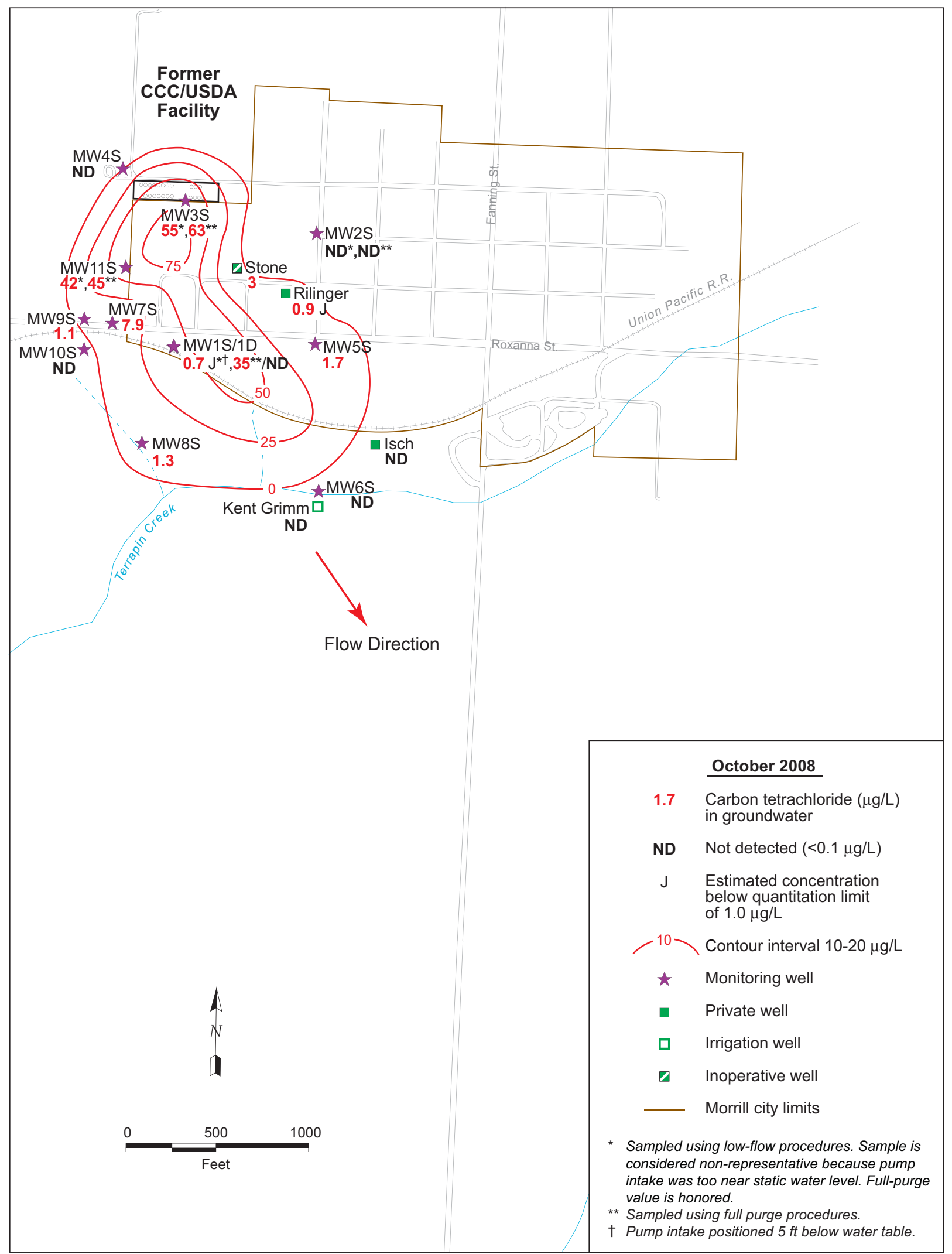

FIGURE 3.5 Lateral extent of the carbon tetrachloride contamination in groundwater at Morrill, as interpreted on the basis of sampling and analysis in October 2008. 


\section{Conclusions and Recommendations}

\subsection{Conclusions}

The findings of the October 2008 monitoring event at Morrill support the following conclusions:

- Measurements of groundwater levels in 2003-2007, made both manually and through the use of automatic recorders, consistently indicated an apparent direction of groundwater flow to the south-southeast from the former CCC/USDA facility. Hand measurements in April 2008, during an extended period of very high water levels in March-July 2008, suggested a potential shift in the groundwater flow direction toward the west. Measurements in September-October 2008, after groundwater elevations had become more consistent with historical conditions, again indicate a groundwater flow direction to the south-southeast.

- Updated hydrographs for July and August 2008 depict frequent drawdown events (as much as approximately $10 \mathrm{ft}$ ) in the water level trace for monitoring well MW6S (located adjacent to Terrapin Creek). These features are likely evidence of pumping of the Grimm irrigation well installed near MW6S in April 2008.

- In monitoring events in 2003-2007, contaminant concentrations at each of the sampled well locations were generally consistent. In the April 2008 sampling event, during an extended period of high water levels and frequent heavy rains, the carbon tetrachloride concentrations at three locations (MW3S, at the southern boundary of the former CCC/USDA facility; MW1S, downgradient from MW3S and along the centerline of the contaminant plume; and MW5S, east of MW1S and near the eastern plume boundary) were significantly decreased. In the October 2008 sampling event, after groundwater elevations returned to levels more consistent with historic conditions, contaminant concentrations also returned to values consistent with those seen in 20032007. 
- No carbon tetrachloride contamination was detected in surface waters or shallow streambed sediments sampled (at the request of the KDHE) at five locations along Terrapin Creek, downgradient from the former CCC/USDA facility.

- Trace concentrations of carbon tetrachloride were detected in samples of branch tissues collected from mature trees at 2 of 24 sampling locations south (downgradient) of the former CCC/USDA facility. The trace detections in vegetation occurred at (1) a mid-plume location south of the former CCC/USDA facility (a new location not previously sampled, near monitoring well MW1S) and (2) an isolated location along Terrapin Creek downstream from monitoring well MW8S, where the contaminant has been detected consistently in groundwater at trace to low levels.

\subsection{Issues and Ongoing Work}

The following issues and recommendations merit consideration in planning for ongoing work at Morrill:

- The significant changes in carbon tetrachloride concentrations, the plume configuration, and apparent groundwater flow directions observed in the April 2008 monitoring event were not confirmed in the October 2008 sampling. Further monitoring is required, however, to evaluate the potential effects on plume dynamics of groundwater level fluctuations and recharge response.

Additional monitoring of the contaminant concentrations is warranted because of the unexplained changes in the concentration of carbon tetrachloride in groundwater, which, in turn, affected the overall plume configuration and the concept of groundwater flow as observed in the April 2008 sampling event. Required monitoring of the Morrill system was initiated in September of 2005 and continued twice yearly until April of 2008. The October 2008 sampling event represented a continuation of that KDHE monitoring requirement. The April 2008 event took place during a period of unusually heavy rainfall, resulting in a corresponding rise in groundwater levels (Section 3.1 and Figure 3.2) and a decrease in contaminant levels, potentially the result of 
dilution or increased groundwater movement and velocity affecting residence time. The return to more normal carbon tetrachloride concentrations and the decrease in groundwater levels to normally anticipated values observed in October 2008 suggest that the April 2008 event represents a statistical abnormality. Therefore, it is considered prudent to continue to monitor the Morrill network to establish whether the results represent a change in conditions or a limited variation due to atypical weather and groundwater conditions.

- Recent automated groundwater monitoring results indicate that pumping of the Grimm irrigation well, installed in April 2008 just south of monitoring well MW6S along Terrapin Creek, had a significant but transient influence on the local groundwater levels at MW6S during the 2008 period of Grimm well operation (July-August). Additional monitoring will be necessary for further determination of the potential wider areal and longer-term hydraulic influence of the Grimm well on groundwater levels and migration patterns in the Morrill study area; such monitoring is recommended.

The importance of the Grimm well lies in the fact that it represents a potentially significant change in groundwater use in the vicinity of Terrapin Creek. Monitoring well MW6S lies adjacent to the creek and almost directly north from the newly installed Grimm production well. Groundwater samples recovered from well MW6S have consistently contained no detectable carbon tetrachloride. A concern is that the high production rates observed for the Grimm well will affect groundwater flow from the north-northwest and across Terrapin Creek, drawing contamination toward the creek from farther upgradient. Results of the April 2008 sampling event (Figure 3.4) illustrate a change in the overall configuration of the carbon tetrachloride plume after the Grimm system came online. The fact that in October 2008, after the irrigation season, the plume configuration and internal contaminant values returned, generally, to previous levels (Figure 3.5) suggests a correlation between the changed plume configuration and water production from the Grimm well.

Although there is presently no way to record water production values or dates for the Grimm well, aside from periodic observations by Argonne 
representatives visiting the site, the responses observed in the hydrograph for well MW6S (Figure 3.2b) contain indications of when the well is pumping. See the last paragraph in Section 3.1 for additional discussion.

- Sampling will continue at the twice yearly frequency previously established under the approved monitoring plan (Argonne 2005b). The twice yearly events will involve sampling of groundwater from the existing network of 12 monitoring wells and 3 private wells, as well as surface waters and stream bed sediments at the 5 established locations along Terrapin Creek.

- The initial comparison of the low-flow purging method and the traditional three-well-volume purging method for the collection of groundwater samples at Morrill gave inconclusive results. The comparative purging and sampling exercise should be repeated during the next (April 2009) monitoring event, with particular attention being given to consistency in the vertical placement of the low-flow pumping system intake relative to the screened interval and static water level positions in each well at the time of sampling.

The CCC/USDA and Argonne should proceed with the comparative sampling evaluation study during the April 2009 sampling event at Morrill. The comparison of sampling techniques and repeatability of results is essential to the recovery of the most accurate and representative groundwater samples. It is imperative that the most appropriate techniques are used consistently at all CCC/USDA investigation sites. Studies like the monitoring program at Morrill are an opportunity for the needed evaluation.

- Sampling of tree branch tissues from existing trees for VOCs analyses has proven to be a valuable indicator of shallow subsurface groundwater contamination. The preliminary detections of carbon tetrachloride in vegetation at the Morrill site to date have been empirically consistent with the previously documented location of the groundwater plume. This interpretation is subject to further confirmation. Vegetation sampling should be continued and expanded as necessary, with the approval of the CCC/USDA; this sampling is a simple, cost-effective method for qualitative monitoring of the 
carbon tetrachloride plume distribution at Morrill, particularly in the downgradient area near Terrapin Creek.

If the results of the ongoing periodic baseline sampling give evidence that contamination is present in the vegetation and/or in the creek itself, the extent of the vegetation sampling will be reevaluated by the CCC/USDA and Argonne to ensure complete coverage of all possible entry points to the creek. Any significant shift in the groundwater plume in close proximity to the creek would also suggest the need for modification of the existing vegetation study area in response to changes in the upgradient plume configuration. 


\section{References}

Alvarado, J.S., and C. Rose, 2004, "Static Headspace Analysis of Volatile Organic Compounds in Soil and Vegetation Samples for Site Characterization,” Talanta 62:17-23.

Argonne, 2002, Final Master Work Plan: Environmental Investigations at Former CCC/USDA Facilities in Kansas, 2002 Revision, ANL/ER/TR-02/004, prepared for the Commodity Credit Corporation, U.S. Department of Agriculture, Washington, D.C., by Argonne National Laboratory, Argonne, Illinois, December.

Argonne, 2004, Final Phase I-Phase II Interim Report: Expedited Site Characterization, Morrill, Kansas, ANL/ER/TR-04/001, prepared for the Commodity Credit Corporation, U.S. Department of Agriculture, Washington, D.C., by Argonne National Laboratory, Argonne, Illinois, December.

Argonne, 2005a, Final Report, Monitoring Well Installation and Sampling, 2004, at Morrill, Kansas, ANL/ER/TR-04/010, prepared for the Commodity Credit Corporation, U.S. Department of Agriculture, Washington, D.C., by Argonne National Laboratory, Argonne, Illinois, November.

Argonne, 2005b, Final Work Plan: Groundwater Monitoring at Morrill, Kansas, ANL/ER/TR05/003, prepared for the Commodity Credit Corporation, U.S. Department of Agriculture, Washington, D.C., by Argonne National Laboratory, Argonne, Illinois, August.

Argonne, 2006a, Draft Report: Groundwater Monitoring at Morrill, Kansas, in September 2005 and March 2006, with Expansion of the Monitoring Network in January 2006, ANL/EVS/AGEM/TR-06-09, prepared for the Commodity Credit Corporation, U.S. Department of Agriculture, Washington, D.C., by Argonne National Laboratory, Argonne, Illinois, September.

Argonne, 2006b, September 2006 Monitoring Results for Morrill, Kansas, ANL/EVS/AGEM/CHRON-1016, prepared for the Commodity Credit Corporation, U.S. Department of Agriculture, Washington, D.C., by Argonne National Laboratory, Argonne, Illinois, December. 
Argonne, 2007a, Reconnaissance Visit along Terrapin Creek, South of the Former CCC/USDA Grain Bin Site and the Exiting Co-op in Morrill, Kansas, January 2007, ANL/EVS/AGEM/CHRON-1042, prepared for the Commodity Credit Corporation, U.S. Department of Agriculture, Washington, D.C., by Argonne National Laboratory, Argonne, Illinois, May 3.

Argonne, 2007b, March-April 2007 Monitoring Results for Morrill, Kansas, ANL/EVS/AGEM/TR-07-06, prepared for the Commodity Credit Corporation, U.S. Department of Agriculture, by Argonne National Laboratory, Argonne, Illinois, May.

Argonne, 2007c, Proposed Addendum to the Groundwater Monitoring Plan for Morrill, Kansas, ANL/EVS/AGEM/CHRON-1042, prepared for the Commodity Credit Corporation, U.S. Department of Agriculture, Washington, D.C., by Argonne National Laboratory, Argonne, Illinois, May 3.

Argonne, 2007d, AGEM-15: Standard Operating Procedure for Sediment Sampling, ANL/EVS/AGEM/CHRON-1042, prepared for the Commodity Credit Corporation, U.S. Department of Agriculture, Washington, D.C., by Argonne National Laboratory, Argonne, Illinois, February 21.

Argonne, 2008a, October 2007 Monitoring Results for Morrill, Kansas, ANL/EVS/AGEM/TR08-02, prepared for the Commodity Credit Corporation, U.S. Department of Agriculture, Washington, D.C., by Argonne National Laboratory, Argonne, Illinois, March.

Argonne, 2008b, Summary of Operations and Performance for the Murdock Site Restoration Project in 2007, ANL/EVS/AGEM/TR-08-07, prepared for the Commodity Credit Corporation, U.S. Department of Agriculture, Washington, D.C., by Argonne National Laboratory, Argonne, Illinois, March.

Argonne, 2008c, April 2008 Monitoring Results for Morrill, Kansas, ANL/EVS/AGEM/TR-0815, prepared for the Commodity Credit Corporation, U.S. Department of Agriculture, Washington, D.C., by Argonne National Laboratory, Argonne, Illinois, July.

EPA, 1995, Method 524.2: Measurement of Purgeable Organic Compounds in Water by Capillary Column Gas Chromatography/Mass Spectrometry, Revision 4.1, edited by 
J.W. Munch, National Exposure Research Laboratory, Office of Research and Development, U.S. Environmental Protection Agency, Cincinnati, Ohio.

KDHE, 2005, letter from C. Carey (Bureau of Environmental Remediation, Kansas Department of Health and Environment, Topeka, Kansas) to C. Roe (Commodity Credit Corporation, U.S. Department of Agriculture, Washington, D.C.) regarding Final Work Plan: Groundwater Monitoring at Morrill, Kansas, October 31.

KDHE, 2007a, letter from C. Carey (Bureau of Environmental Remediation, Kansas Department of Health and Environment, Topeka, Kansas) to C. Roe (Commodity Credit Corporation, U.S. Department of Agriculture, Washington, D.C.) regarding Draft Report: Groundwater Monitoring at Morrill, Kansas, in September 2005 and March 2006, with Expansion of the Monitoring Network in January 2006, January 5.

KDHE, 2007b, letter from C. Carey (Bureau of Environmental Remediation, Kansas Department of Health and Environment, Topeka, Kansas) to C. Roe (Commodity Credit Corporation, U.S. Department of Agriculture, Washington, D.C.) regarding March-April 2007 Monitoring Results for Morrill, Kansas, August 1.

KDHE, 2008, letter from E. McWilliams (Bureau of Environmental Remediation, Kansas Department of Health and Environment, Topeka, Kansas) to C. Roe (Commodity Credit Corporation, U.S. Department of Agriculture, Washington, D.C.) regarding April 2008 Monitoring Report for Morrill, Kansas, August 6.

Puls, R.W., and Barcelona, M.J., 1996, "Low-Flow (Minimal Drawdown) Ground-Water Sampling Procedures,” EPA/540/S-95/504, in Ground Water Issue, Superfund Technology Support Center for Ground Water, National Risk Management Research Laboratory, Ada, Oklahoma, April (www.epa.gov/tio/tsp/download/lwflw2a.pdf).

Yeskis, D., and B. Zavala, 2002, Ground-Water Sampling Guidelines for Superfund and RCRA Project Managers: Ground Water Forum Issue Paper, EPA 542-S-02-001, Technology Innovative Office, Office of Solid Waste and Emergency Response, U.S. Environmental Protection Agency, Washington, D.C., May (http://www.epa.gov/tio/tsp/download/ gw_sampling_guide.pdf). 


\section{Appendix A:}

Sequence of Sampling Activities at Morrill, Kansas, in October 2008 
TABLE A.1 Sequence of sampling activities in July 2008 and October 2008 at Morrill, Kansas.

\begin{tabular}{|c|c|c|c|c|c|c|c|c|c|}
\hline $\begin{array}{l}\text { Sample } \\
\text { Date }\end{array}$ & Time & Sample & Medium & Type $^{a}$ & Location & $\begin{array}{l}\text { Depth } \\
\text { (ft BGL) }\end{array}$ & $\begin{array}{l}\text { Chain of } \\
\text { Custody }\end{array}$ & $\begin{array}{l}\text { Shipment } \\
\text { Date }\end{array}$ & Sample Description \\
\hline \multicolumn{10}{|c|}{ Vegetation sampling in July 2008} \\
\hline $7 / 24 / 08$ & $14: 30$ & MR003-B-16620 & Veg & Branch & MR003 & - & 4345 & $7 / 24 / 08$ & $\begin{array}{l}\text { Willow identified by global positioning system } \\
\text { (GPS). Stake not found. Sample collected from } \\
\text { secondary branch from trunk lying horizontally, } \\
6 \mathrm{ft} \text { above ground, north orientation. }\end{array}$ \\
\hline $7 / 24 / 08$ & $14: 45$ & MR004-B-16619 & Veg & Branch & MR004 & - & 4345 & $7 / 24 / 08$ & $\begin{array}{l}\text { Large willow on south side of creek, very close to } \\
\text { water. Found prior marker from } 2007 \text {. Sample } \\
\text { collected } 3 \mathrm{ft} \text { above ground, south orientation. }\end{array}$ \\
\hline $7 / 24 / 08$ & $14: 55$ & MR002-B-16621 & Veg & Branch & MR002 & - & 4345 & $7 / 24 / 08$ & $\begin{array}{l}\text { Willow on north side of creek. Ribbon and stake } \\
\text { found. Primary branch } 6 \mathrm{ft} \text { above ground, south } \\
\text { orientation. }\end{array}$ \\
\hline $7 / 24 / 08$ & $14: 56$ & MR001A-B-16622 & Veg & Branch & MR001A & - & 4345 & $7 / 24 / 08$ & $\begin{array}{l}\text { Large willow between MR001 and MR002. Sample } \\
\text { collected } 6 \mathrm{ft} \text { above ground, south orientation. }\end{array}$ \\
\hline $7 / 24 / 08$ & $15: 10$ & MR001-B-16623 & Veg & Branch & MR001 & - & 4345 & $7 / 24 / 08$ & $\begin{array}{l}\text { Willow previously sampled as MR001 with dead } \\
\text { top near burn pit. Identified by GPS. Sample } \\
\text { collected } 8 \mathrm{ft} \text { above ground, east orientation. }\end{array}$ \\
\hline $7 / 24 / 08$ & $15: 15$ & MR005-B-16618 & Veg & Branch & MR005A & - & 4345 & $7 / 24 / 08$ & $\begin{array}{l}\text { Maple previsouly sampled as MR005 not } \\
\text { accessable. Sampled willow (18-in. diameter) to } \\
\text { the west of location on north side of creek. } \\
\text { Sample collected } 6 \mathrm{ft} \text { above ground, south } \\
\text { orientation. }\end{array}$ \\
\hline $7 / 24 / 08$ & $15: 30$ & MR013-B-16624 & Veg & Branch & MR013 & - & 4345 & $7 / 24 / 08$ & $\begin{array}{l}\text { Willow. Previous marker and stake found. Sample } \\
\text { collected } 8 \mathrm{ft} \text { above ground, south orientation. }\end{array}$ \\
\hline $7 / 24 / 08$ & $15: 35$ & MR006-B-16625 & Veg & Branch & MR006 & - & 4345 & $7 / 24 / 08$ & $\begin{array}{l}\text { Large cottonwood. Previous stake found. Sample } \\
\text { collected } 7 \mathrm{ft} \text { above ground, east orientation. }\end{array}$ \\
\hline $7 / 24 / 08$ & $15: 40$ & MR007-B-16626 & Veg & Branch & MR007 & - & 4345 & $7 / 24 / 08$ & $\begin{array}{l}\text { Large willow near monitoring well. Found MW but } \\
\text { not tree stake. Sample collected } 5 \mathrm{ft} \text { above } \\
\text { ground, east orientation. }\end{array}$ \\
\hline $7 / 24 / 08$ & $15: 45$ & MR008-B-16627 & Veg & Branch & MR008 & - & 4367 & $7 / 24 / 08$ & $\begin{array}{l}\text { Large cottonwood. Found previous stake. Fourth- } \\
\text { order branch, } 8 \mathrm{ft} \text { from main stem. Sample } \\
\text { collected } 10 \mathrm{ft} \text { above ground, east orientation. }\end{array}$ \\
\hline $7 / 24 / 08$ & $15: 46$ & MR014-B-16628 & Veg & Branch & MR014 & - & 4345 & $7 / 24 / 08$ & $\begin{array}{l}\text { Large willow. Previous stake found. Primary } \\
\text { branch } 5 \mathrm{ft} \text { above ground, east orientation. }\end{array}$ \\
\hline $7 / 24 / 08$ & $15: 47$ & MR009-B-16629 & Veg & Branch & MR009 & - & 4367 & $7 / 24 / 08$ & $\begin{array}{l}\text { Three-stemmed willow. Old stake found. Primary } \\
\text { branch } 5 \mathrm{ft} \text { above ground, west orientation. }\end{array}$ \\
\hline $7 / 24 / 08$ & $15: 50$ & MR015-B-16630 & Veg & Branch & MR015 & - & 4367 & $7 / 24 / 08$ & $\begin{array}{l}\text { Mulberry. Previous stake found. Sample collected } \\
3 \mathrm{ft} \text { above ground, east orientation. }\end{array}$ \\
\hline
\end{tabular}


TABLE A.1 (Cont.)

\begin{tabular}{|c|c|c|c|c|c|c|c|c|c|}
\hline $\begin{array}{c}\text { Sample } \\
\text { Date }\end{array}$ & Time & Sample & Medium & Type $^{\mathrm{a}}$ & Location & $\begin{array}{l}\text { Depth } \\
\text { (ft BGL) }\end{array}$ & $\begin{array}{l}\text { Chain of } \\
\text { Custody }\end{array}$ & $\begin{array}{l}\text { Shipment } \\
\text { Date }\end{array}$ & Sample Description \\
\hline
\end{tabular}

Vegetation sampling in July 2008 (cont.)

\begin{tabular}{|c|c|c|c|c|c|c|c|c|c|}
\hline $7 / 24 / 08$ & $15: 51$ & MR010-B-16631 & Veg & Branch & MR010 & - & 4367 & $7 / 24 / 08$ & $\begin{array}{l}\text { Large elm, decapitated. Previous stake found. } \\
\text { Sample collected } 6 \mathrm{ft} \text { above ground, east } \\
\text { orientation. }\end{array}$ \\
\hline $7 / 24 / 08$ & $15: 55$ & MR011-B-16632 & Veg & Branch & MR011 & - & 4367 & $7 / 24 / 08$ & $\begin{array}{l}\text { Osage orange. Found old stake. Sample collected } \\
6 \mathrm{ft} \text { above ground, north orientation. }\end{array}$ \\
\hline $7 / 24 / 08$ & $16: 00$ & MR012-B-16633 & Veg & Branch & MR012 & - & 4367 & $7 / 24 / 08$ & $\begin{array}{l}\text { Large willow with one large stem lying across the } \\
\text { stream bank Previous stake found. Primary } \\
\text { branch sampled approximately } 10 \mathrm{ft} \text { above } \\
\text { ground, east orientation. }\end{array}$ \\
\hline $7 / 24 / 08$ & 16:05 & MR016-B-16634 & Veg & Branch & MR016 & - & 4367 & $7 / 24 / 08$ & $\begin{array}{l}\text { Willow with one large stem lying over creek. Old } \\
\text { ribbon found. Sample collected approximately } \\
15 \mathrm{ft} \text { above ground, north orientation. }\end{array}$ \\
\hline $7 / 24 / 08$ & $16: 10$ & MR017-B-16635 & Veg & Branch & MR017 & - & 4367 & $7 / 24 / 08$ & $\begin{array}{l}\text { Small willow appproximately } 10 \mathrm{~cm} \text { in diameter. } \\
\text { Old stake found. Sample collected from } \\
\text { secondary branch } 3 \mathrm{ft} \text { above ground, west } \\
\text { orientation. }\end{array}$ \\
\hline $7 / 24 / 08$ & $16: 15$ & MR018-B-16636 & Veg & Branch & MR018 & - & 4367 & $7 / 24 / 08$ & $\begin{array}{l}\text { Osage orange. Old stake found. Sample collected } \\
\text { from tertiary branch } 5 \mathrm{ft} \text { from main stem, } 10 \mathrm{ft} \\
\text { above ground, north orientation. }\end{array}$ \\
\hline $7 / 24 / 08$ & $16: 30$ & MR019-B-16637 & Veg & Branch & MR019 & - & 4367 & $7 / 24 / 08$ & $\begin{array}{l}\text { Large silver maple located on Roxanne Avenue } \\
\text { between railroad and pump house. Sample } \\
\text { collected from secondary branch, } 6 \mathrm{ft} \text { from main } \\
\text { stem, } 8 \mathrm{ft} \text { above ground, east orientation. }\end{array}$ \\
\hline $7 / 24 / 08$ & $16: 40$ & MR020-B-20021 & Veg & Branch & MR020 & - & 4367 & $7 / 24 / 08$ & $\begin{array}{l}\text { Ash tree on south side of Roxanne Street between } \\
\text { houses numbered } 104 \text { and } 106 \text {. Sample } \\
\text { collected } 6 \mathrm{ft} \text { above ground, west orientation. }\end{array}$ \\
\hline $7 / 24 / 08$ & $16: 45$ & MR021-B-20022 & Veg & Branch & MR021 & - & 4367 & $7 / 24 / 08$ & $\begin{array}{l}\text { Juniper to the east of the south row of bins on } \\
\text { former CCC/USDA property, east of bin \#20. } \\
\text { Third-order branch, } 2 \mathrm{ft} \text { from main stem. } \\
\text { Sampled } 3 \mathrm{ft} \text { above ground, east orientation. }\end{array}$ \\
\hline $7 / 24 / 08$ & $16: 46$ & MR022-B-20023 & Veg & Branch & MR022 & - & 4367 & $7 / 24 / 08$ & $\begin{array}{l}\text { Hemp growing near bin \#20 at former CCC/USDA } \\
\text { facility. Sample collected at ground level. }\end{array}$ \\
\hline $7 / 24 / 08$ & $16: 50$ & MR023-B-20024 & Veg & Branch & MR023 & - & 4367 & $7 / 24 / 08$ & $\begin{array}{l}\text { Hackberry growing near bin \#20. Sample collected } \\
\text { from base sucker. }\end{array}$ \\
\hline
\end{tabular}


TABLE A.1 (Cont.)

\begin{tabular}{|c|c|c|c|c|c|c|c|c|c|}
\hline $\begin{array}{l}\text { Sample } \\
\text { Date }\end{array}$ & Time & Sample & Medium & Type $^{a}$ & Location & $\begin{array}{l}\text { Depth } \\
\text { (ft BGL) }\end{array}$ & $\begin{array}{l}\text { Chain of } \\
\text { Custody }\end{array}$ & $\begin{array}{l}\text { Shipment } \\
\text { Date }\end{array}$ & Sample Description \\
\hline \multicolumn{10}{|c|}{ Vegetation sampling in July 2008 (cont.) } \\
\hline $7 / 24 / 08$ & $17: 00$ & MR024-B-20025 & Veg & Branch & MR024 & - & 4367 & $7 / 24 / 08$ & $\begin{array}{l}\text { Black walnut east of new metal building on the } \\
\text { former CCC/USDA property. Secondary branch, } \\
2 \mathrm{ft} \text { from main stem. Sampled } 3 \mathrm{ft} \text { above ground, } \\
\text { south orientation. }\end{array}$ \\
\hline \multicolumn{10}{|c|}{ October 2008 monitoring } \\
\hline 10/20/08 & $14: 12$ & MRMW7S-W-27627 & Water & MW & MW7S & $20-45$ & 6147 & 10/21/08 & $\begin{array}{l}\text { Depth to water }=17.2 \mathrm{ft} \text {. Depth of } 4 \text {-in. well }=47 \mathrm{ft} \text {. } \\
\text { Sample collected by using low-flow bladder } \\
\text { pump after purging of of } 6.3 \mathrm{~L} \text {. Pump intake } \\
\text { positioned at } 32.5 \mathrm{ft} \text {. }\end{array}$ \\
\hline $10 / 20 / 08$ & $14: 32$ & MRMW9S-W-27629 & Water & MW & MW9S & $\begin{array}{l}38.83- \\
53.83\end{array}$ & 6147 & 10/21/08 & $\begin{array}{l}\text { Depth to water }=21.5 \mathrm{ft} \text {. Depth of } 2 \text {-in. well = } \\
58.5 \mathrm{ft} \text {. Sample collected by using low-flow } \\
\text { bladder pump after purging of } 11 \mathrm{~L} \text {. Pump intake } \\
\text { positioned at } 46.33 \mathrm{ft} \text {. }\end{array}$ \\
\hline 10/20/08 & $15: 51$ & MRMW10S-W-27630 & Water & MW & MW10S & $30-45$ & 6147 & $10 / 21 / 08$ & $\begin{array}{l}\text { Depth to water = } 13.7 \mathrm{ft} \text {. Depth of } 2 \text {-in. well = } \\
49.7 \mathrm{ft} \text {. Sample collected by using low-flow } \\
\text { bladder pump after purging of } 5.4 \mathrm{~L} \text {. Pump } \\
\text { intake positioned at } 37.5 \mathrm{ft} \text {. }\end{array}$ \\
\hline 10/20/08 & $15: 52$ & MRMW11S-W-27631 & Water & MW & MW11S & $53-68$ & 6147 & $10 / 21 / 08$ & $\begin{array}{l}\text { Depth to water }=37.1 \mathrm{ft} \text {. Depth of } 2 \text {-in. well }= \\
72.7 \mathrm{ft} \text {. Sample collected by using low-flow } \\
\text { bladder pump after purging of } 9 \mathrm{~L} \text {. Pump intake } \\
\text { positioned at } 60.5 \mathrm{ft} \text {. }\end{array}$ \\
\hline 10/20/08 & $16: 50$ & MRSMB-W-27648 & Water & Creek & SMB & - & 6147 & $10 / 21 / 08$ & Water sample from Terrapin Creek. \\
\hline 10/20/08 & $16: 51$ & MRSMB-S-27648 & Sediment & Creek & SMB & - & 6149 & $10 / 21 / 08$ & Sediment sample from Terrapin Creek. \\
\hline 10/20/08 & $16: 56$ & MRMW6S-W-27626 & Water & MW & MW6S & $10-25$ & 6147 & $10 / 21 / 08$ & $\begin{array}{l}\text { Depth to water }=5.7 \mathrm{ft} \text {. Depth of } 4 \text {-in. well }=26.9 \mathrm{ft} \text {. } \\
\text { Sample collected by using low-flow bladder } \\
\text { pump after purging of } 5 \mathrm{~L} \text {. Pump intake } \\
\text { positioned at } 17.5 \mathrm{ft} \text {. }\end{array}$ \\
\hline 10/20/08 & $17: 16$ & MRMW8S-W-27628 & Water & MW & MW8S & $10-25$ & 6147 & 10/21/08 & $\begin{array}{l}\text { Depth to water }=3.6 \mathrm{ft} \text {. Depth of } 4 \text {-in. well }=26.8 \mathrm{ft} \text {. } \\
\text { Sample collected by using low-flow bladder } \\
\text { pump after purging of } 8 \mathrm{~L} \text {. Pump intake } \\
\text { positioned at } 17.5 \mathrm{ft} \text {. }\end{array}$ \\
\hline 10/20/08 & $17: 18$ & MRSM1-W-27644 & Water & Creek & SM1 & - & 6147 & $10 / 21 / 08$ & Water sample from Terrapin Creek. \\
\hline 10/20/08 & $17: 19$ & MRSM1-S-27644 & Sediment & Creek & SM1 & - & 6149 & $10 / 21 / 08$ & Sediment sample from Terrapin Creek. \\
\hline 10/20/08 & $17: 40$ & MRSM2-W-27645 & Water & Creek & $\mathrm{SM} 2$ & - & 6147 & $10 / 21 / 08$ & Water sample from Terrapin Creek. \\
\hline
\end{tabular}


TABLE A.1 (Cont.)

\begin{tabular}{|c|c|c|c|c|c|c|c|c|c|}
\hline $\begin{array}{l}\text { Sample } \\
\text { Date }\end{array}$ & Time & Sample & Medium & Type $^{a}$ & Location & $\begin{array}{l}\text { Depth } \\
\text { (ft BGL) }\end{array}$ & $\begin{array}{l}\text { Chain of } \\
\text { Custody }\end{array}$ & $\begin{array}{l}\text { Shipment } \\
\text { Date }\end{array}$ & Sample Description \\
\hline \multicolumn{10}{|c|}{ October 2008 monitoring (cont.) } \\
\hline $10 / 20 / 08$ & $17: 41$ & MRSM2-S-27645 & Sediment & Creek & SM2 & - & 6149 & $10 / 21 / 08$ & Sediment sample from Terrapin Creek. \\
\hline $10 / 20 / 08$ & $17: 48$ & MRTD12-W-27635 & Water & Creek & TD12 & $27-67$ & 6147 & 10/21/08 & Collected water flowing out of tile drain into creek. \\
\hline $10 / 20 / 08$ & $18: 16$ & MRSM3-W-27646 & Water & Creek & SM3 & - & 6147 & 10/21/08 & Water sample from Terrapin Creek. \\
\hline $10 / 20 / 08$ & $18: 17$ & MRSM3-S-27646 & Sediment & Creek & SM3 & - & 6149 & $10 / 21 / 08$ & Sediment sample from Terrapin Creek. \\
\hline $10 / 20 / 08$ & $18: 21$ & MRMW1S-W-27620 & Water & MW & MW1S & $11-51$ & 6147 & 10/21/08 & $\begin{array}{l}\text { Depth to water }=25.8 \mathrm{ft} \text {. Depth of } 4 \text {-in. well }=54 \mathrm{ft} \text {. } \\
\text { Sample collected by using low-flow bladder } \\
\text { pump after purging of } 6 \mathrm{~L} \text {. Pump intake } \\
\text { positioned at } 31 \mathrm{ft} \text {. }\end{array}$ \\
\hline $10 / 20 / 08$ & $18: 24$ & MRSM4-W-27647 & Water & Creek & SM4 & - & 6147 & 10/21/08 & Water sample from Terrapin Creek. \\
\hline $10 / 20 / 08$ & $18: 25$ & MRSM4-S-27647 & Sediment & Creek & SM4 & - & 6149 & 10/21/08 & Sediment sample from Terrapin Creek. \\
\hline $10 / 20 / 08$ & $18: 30$ & MRMW4S-W-27624 & Water & MW & MW4S & $17-47$ & 6147 & 10/21/08 & $\begin{array}{l}\text { Depth to water }=36.7 \mathrm{ft} \text {. Depth of } 4 \text {-in. well }= \\
47.8 \mathrm{ft} \text {. Sample collected by using bailer after } \\
\text { purging of } 8 \text { gal. Purged one well volume and } \\
\text { sampled. }\end{array}$ \\
\hline $10 / 20 / 08$ & $19: 31$ & MRMW1D-W-27621 & Water & MW & MW1D & $63-88$ & 6147 & $10 / 21 / 08$ & $\begin{array}{l}\text { Depth to water }=30.4 \mathrm{ft} \text {. Depth of } 4 \text {-in. well }=89 \mathrm{ft} \text {. } \\
\text { Sample collected by using low-flow bladder } \\
\text { pump after purging of } 7 \mathrm{~L} \text {. Pump intake } \\
\text { positioned at } 75.5 \mathrm{ft} \text {. }\end{array}$ \\
\hline $10 / 21 / 08$ & $8: 43$ & MRMW5S-W-27625 & Water & MW & MW5S & $15-55$ & 6148 & 10/21/08 & $\begin{array}{l}\text { Depth to water }=22.5 \mathrm{ft} \text {. Depth of } 4 \text {-in. well = } \\
54.6 \mathrm{ft} \text {. Sample collected by using low-flow } \\
\text { bladder pump after purging of } 7 \mathrm{~L} \text {. Pump intake } \\
\text { positioned at } 35 \mathrm{ft} \text {. }\end{array}$ \\
\hline $10 / 21 / 08$ & $9: 25$ & MRMW3S-W-27623 & Water & MW & MW3S & $18-48$ & 6148 & $10 / 21 / 08$ & $\begin{array}{l}\text { Depth to water }=27 \mathrm{ft} \text {. Depth of } 4 \text {-in. well }=47.8 \mathrm{ft} \text {. } \\
\text { Sample collected by using low-flow bladder } \\
\text { pump after purging of } 42 \mathrm{~L} \text {. Pump intake } \\
\text { positioned at } 33 \mathrm{ft} \text {. }\end{array}$ \\
\hline $10 / 21 / 08$ & $9: 49$ & MRQCIR-W-27641 & Water & $\mathrm{RI}$ & QC & - & 6148 & $10 / 21 / 08$ & $\begin{array}{l}\text { Rinsate of decontaminated sampling line after } \\
\text { collection of sample MRMW3S-W-27623. }\end{array}$ \\
\hline $10 / 21 / 08$ & $10: 13$ & MRMW2S-W-27652 & Water & MW & MW2S & $13-53$ & 6148 & $10 / 21 / 08$ & $\begin{array}{l}\text { Purged three well volumes with bailer and } \\
\text { sampled. }\end{array}$ \\
\hline $10 / 21 / 08$ & $11: 10$ & MRMW11S-W-27651 & Water & MW & MW11S & $53-68$ & 6148 & 10/21/08 & $\begin{array}{l}\text { Purged three well volumes with Redi-Flo pump; } \\
\text { then sampled at low flow. Pump intake position } \\
\text { not recorded. }\end{array}$ \\
\hline
\end{tabular}


TABLE A.1 (Cont.)

\begin{tabular}{|c|c|c|c|c|c|c|c|c|c|}
\hline $\begin{array}{l}\text { Sample } \\
\text { Date }\end{array}$ & Time & Sample & Medium & Type $^{\mathrm{a}}$ & Location & $\begin{array}{l}\text { Depth } \\
\text { (ft BGL) }\end{array}$ & $\begin{array}{l}\text { Chain of } \\
\text { Custody }\end{array}$ & $\begin{array}{l}\text { Shipment } \\
\text { Date }\end{array}$ & Sample Description \\
\hline \multicolumn{10}{|c|}{ October 2008 monitoring (cont.) } \\
\hline 10/21/08 & $12: 02$ & MRMW2S-W-27622 & Water & MW & MW2S & $13-53$ & 6148 & $10 / 21 / 08$ & $\begin{array}{l}\text { Depth to water }=33.7 \mathrm{ft} \text {. Depth of } 4 \text {-in. well = } \\
53.5 \mathrm{ft} \text {. Sample collected by using low-flow } \\
\text { bladder pump after purging of } 5 \mathrm{~L} \text {. Pump intake } \\
\text { positioned at } 33 \mathrm{ft} \text {. }\end{array}$ \\
\hline 10/21/08 & $12: 04$ & MRMW3S-W-27650 & Water & MW & MW3S & $18-48$ & 6148 & $10 / 21 / 08$ & $\begin{array}{l}\text { Purged three well volumes with Redi-Flo pump; } \\
\text { then sampled at low flow. Pump intake position } \\
\text { not recorded. }\end{array}$ \\
\hline 10/21/08 & $12: 39$ & MRRILLINGER-W-27633 & Water & DW & Rillinger & - & 6148 & $10 / 21 / 08$ & $\begin{array}{l}\text { Allowed pump to run for } 5 \text { min; then sampled from } \\
\text { tap. }\end{array}$ \\
\hline 10/21/08 & $12: 56$ & MRQCIR-W-27642 & Water & $\mathrm{RI}$ & QC & - & 6148 & 10/21/08 & $\begin{array}{l}\text { Rinsate of decontaminated sampling line after } \\
\text { collection of sample MRMW3S-W-27650. }\end{array}$ \\
\hline 10/21/08 & $13: 00$ & MRSTONE-W-27634 & Water & DW & Stone & 43 & 6148 & $10 / 21 / 08$ & Bailed $5 \mathrm{gal}$; then sampled with bailer. \\
\hline 10/21/08 & 13:06 & MRMW1S-W-27649 & Water & MW & MW1S & $11-51$ & 6148 & $10 / 21 / 08$ & $\begin{array}{l}\text { Purged } 3 \text { well volumes with Redi-Flo pump; then } \\
\text { sampled at low flow. Pump intake position not } \\
\text { recorded. }\end{array}$ \\
\hline 10/21/08 & $14: 15$ & MRISCH-W-27632 & Water & DW & Isch & - & 6148 & 10/21/08 & $\begin{array}{l}\text { Allowed pump to run for } 5 \mathrm{~min} \text {; then sampled from } \\
\text { tap. }\end{array}$ \\
\hline 10/21/08 & $17: 00$ & MRQCTB-W-27638 & Water & TB & QC & - & 6148 & $10 / 21 / 08$ & $\begin{array}{l}\text { Trip blank sent to the AGEM Laboratory for organic } \\
\text { analysis with water samples listed on chain-of- } \\
\text { custody forms } 6147 \text { and } 6148 .\end{array}$ \\
\hline
\end{tabular}

a Sample type abbreviations: DW, domestic well; MW, monitoring well; RI, rinsate; TB, trip blank. 


\section{Appendix B:}

\section{Analytical Results for Waste Purge Water}




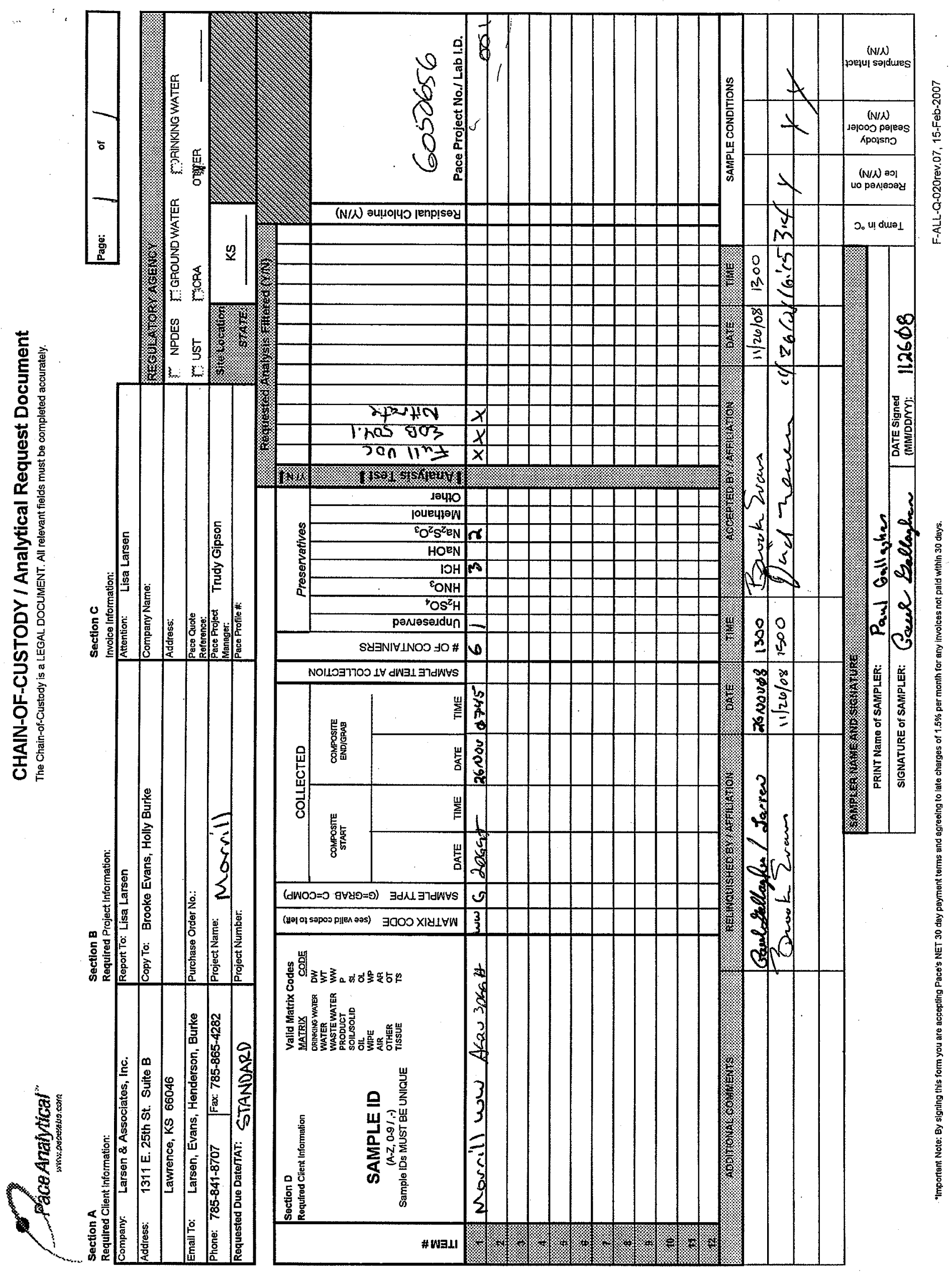


Sample Condition Upon Receipt

PaceAnalytical

Client Name: $6 x^{2}$

Project\#_6050656

Optional Proj. Due Date: 12110108

Courier: $\square$ Fed Ex $\square$ UPS $\square$ USPS $\tilde{\sigma}_{\text {Client }} \square$ Commercial $\square$ Pace Other Tracking \#:

Custody Seal on Cooler/Box Present: 6 yes $\square$ no Seals intact: $\measuredangle$ yes $\square$ no Proj. Name: Morrill

Packing Material: $\square$ Bubble Wrap $\square$ Bubble Bags $\square$ None $B$ Other far

Thermometer Used

Cooler Temperature

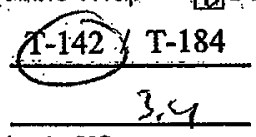

Type of Ice: We Blue Non

$\square$ Samples on ice, cooling process has begun

Temp should be above freezing to $6^{\circ} \mathrm{C}$

Biological Tissue is Frozen: Yes No

Chain of Custody Present Comments:

Chain of Custody Filled Out:

Chain of Custody Relinquished:

Sampler Name \& Signature on COC:

Samples Arrived within Hold Time:

Short Hold Time Analysis (<72hr):

Rush Turn Around Time Requested:

Gres ano DN/A 1.

Gres ano DN/A 2.

Sufficient Volume:

GYes $\square$ No $\square \mathrm{N} / \mathrm{A} / 3$

Correct Containers Used:

Gres $\square$ No $\square$ N/A 4 .

-Pace Containers Used:

GYes पNo DN/A 5.

Containers Intact: $\square$ Yes LiNo $_{\text {NN/A }} 6$.

Filtered volume received for Dissolved tests

Sample Labels match COC:

口Yes GNo [N/A 7.

GYes $\square$ No $\square$ N/A 8.

-Includes date/time/ID/Analysis Matrix: LT

All containers needing preservation have been checked.

All containers needing preservation are found to be in compliance with EPA recommendation.

exceptions: $v \not A$, collform, TOC, O\&G, WI-DRO (water) TYes $\square$ No $\square$ N/A 9.

Eres $\square$ No $\square$ N/A

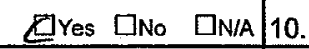

Samples checked for dechlorination:

Headspace in VOA Vials ( $>6 \mathrm{~mm}$ ):

DYes [No ZM/A 11.

Trip Blank Present:

Trip Blank Custody Seals Present

Pace Trip Blank Lot \# (if purchased):

Client Notification/ Resolution:

Person Contacted:

Field Data Required?

$\mathrm{Y} / \mathrm{N}$

Comments/ Resolution: Date/Time:

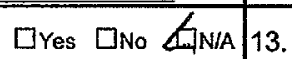

$\square$ Yes $\square$ No $/$ N/A

\begin{tabular}{l|l|l|l|l}
\hline LTes $\square$ Ino & Lot \# of added when
\end{tabular}

DYes DNo GN/A 14.

$\square$ Yes GNo $\square$ N/A 15.

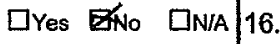

$\square$ Yes $\square$ No RSNA contents serentecter

\section{Project Manager Review: thas 11.28 .08}

Date:

Note: Whenever there is a discrepancy affecting North Carolina compliance samples, a copy of this form will be sent to the North Carolina DEHNR Certification Office (i.e out of hold, incorrect preservative, out of temp, incorrect containers) 
December 10, 2008

Lisa Larsen

Larsen \& Associates, Inc.

1311 E. 25th St.

Suite $B$

Lawrence, KS 66046

\section{RE: Project: Morrill}

Pace Project No.: 6050656

Dear Lisa Larsen:

Enclosed are the analytical results for sample(s) received by the laboratory on November 26, 2008. The results relate only to the samples included in this report. Results reported herein conform to the most current NELAC standards, where applicable, unless otherwise narrated in the body of the report.

If you have any questions concerning this report, please feel free to contact me.

Sincerely,

$$
\text { suedy sigion }
$$

Trudy Gipson

trudy.gipson@pacelabs.com

Project Manager

\section{Enclosures}

cc: Holly Burke, Larsen \& Associates, Inc. Brooke Evans, Larsen \& Associates, Inc. 
$\beta^{2}$

Pace Analytical Services, Inc. 9608 Loiret BIvd.

Lenexa, KS 66219

(913)599-5665

\title{
CERTIFICATIONS
}

\author{
Project: Morrill \\ Pace Project No: $\quad 6050656$
}

Kansas Certification IDs

Utah Certification Number: 9135995665

Texas Certification Number: T104704407-08-TX

Oklahoma Certification Number: $9205 / 9935$

Nevada Certification Number: KS000212008A

Louisiana Certification Number: 03055 lowa Certification Number: 118

Illinois Certification Number: 001191

Arkansas Certification Number: 05-008-0

A2LA Certification Number: 2456.01

Kansas/NELAP Certification Number: E-10116

This report shall not be reproduced, except in full,

without the written consent of Pace Analytical Services, Inc..

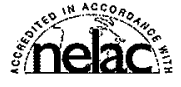



9608 Loiret Blvd.

Lenexa, KS 66219 (913)599-5665

SAMPLE SUMMARY

$\begin{array}{ll}\text { Project: } & \text { Morrill } \\ \text { Pace Project No.: } & 6050656\end{array}$

\begin{tabular}{|c|c|c|c|c|}
\hline Lab ID & Sample ID & Matrix & Date Collected & Date Received \\
\hline 6050656001 & MORRILL WW & Water & $11 / 26 / 08 \quad 07: 45$ & $11 / 26 / 0816: 15$ \\
\hline
\end{tabular}


SAMPLE ANALYTE COUNT

Project: Morrill

Pace Project No.: 6050656

\begin{tabular}{|c|c|c|c|c|}
\hline Lab ID & Sample ID & Method & Analysts & $\begin{array}{l}\text { Analytes } \\
\text { Reported }\end{array}$ \\
\hline \multirow[t]{3}{*}{6050656001} & MORRILL WW & EPA 300.0 & AJM & 1 \\
\hline & & EPA 5030B/8260 & AJA & 71 \\
\hline & & EPA 504.1 & SRM & 1 \\
\hline
\end{tabular}




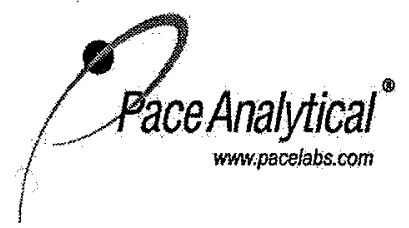

Pace Analytical Services, Inc. 9608 Loiret Blvd.

Lenexa, KS 66219

(913)599-5665

\section{ANALYTICAL RESULTS}

$\begin{array}{ll}\text { Project: } & \text { Morrill } \\ \text { Pace Project No. } & 6050656\end{array}$

Sample: MORRILL WW $\quad$ Lab ID: $6050656001 \quad$ Collected: 11/26/08 07:45 Received: 11/26/08 16:15 Matrix: Water

$\frac{\text { Parameters }}{504 \text { GCS EDB and DBCP }} \frac{\text { Results }}{\text { Analytical Method: EPA 504.1 Preparation Method: EPA 504.1 }} \frac{\text { Units }}{\text { Report Limit }} \frac{\text { DF }}{\text { Prepared }}$

1,2-Dibromoethane (EDB)

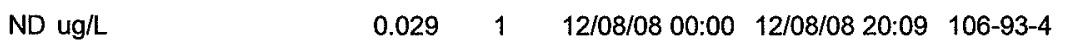

8260 MSV

Analytical Method: EPA 5030B/8260

Acetone

Benzene

Bromobenzene

Bromochloromethane

Bromodichloromethane

Bromoform

Bromomethane

TOTAL BTEX

2-Butanone (MEK)

n-Butylbenzene

sec-Butylbenzene

tert-Butylbenzene

Carbon disulfide

Carbon tetrachloride

Chlorobenzene

Chloroethane

Chloroform

Chloromethane

2-Chlorotoluene

4-Chlorotoluene

1,2-Dibromo-3-chloropropane

Dibromochloromethane

1,2-Dibromoethane (EDB)

Dibromomethane

1,2-Dichlorobenzene

1,3-Dichlorobenzene

1,4-Dichlorobenzene

Dichlorodifluoromethane

1,1-Dichloroethane

1,2-Dichloroethane

1,2-Dichloroethene (Total)

1,1-Dichloroethene

cis-1,2-Dichloroethene

trans-1,2-Dichloroethene

1,2-Dichloropropane

1,3-Dichloropropane

2,2-Dichloropropane

1,1-Dichloropropene

cis-1,3-Dichloropropene

trans-1,3-Dichloropropene

Ethylbenzene

Hexachloro-1,3-butadiene

2-Hexanone

Isopropylbenzene (Cumene)

Date: 12/10/2008 02:58 PM

\begin{tabular}{|c|c|c|c|c|}
\hline ND ug/L & 10.0 & 1 & $12 / 08 / 0815: 10$ & $67-64-1$ \\
\hline ND ug/L & 1.0 & 1 & $12 / 08 / 0815: 10$ & $71-43-2$ \\
\hline ND ug/L & 1.0 & 1 & 12/08/08 15:10 & $108-86-1$ \\
\hline ND ug/L & 1.0 & 1 & $12 / 08 / 0815: 10$ & $74-97-5$ \\
\hline ND ug/L & 1.0 & 1 & $12 / 08 / 0815: 10$ & $75-27-4$ \\
\hline ND ug/L & 1.0 & 1 & $12 / 08 / 0815: 10$ & $75-25-2$ \\
\hline $6.0 \mathrm{ug} / \mathrm{L}$ & 1.0 & 1 & $12 / 08 / 08 \quad 15: 10$ & $74-83-9$ \\
\hline ND ug/L & 1.0 & 1 & $12 / 08 / 08 \quad 15: 10$ & \\
\hline ND ug/L & 10.0 & 1 & $12 / 08 / 08$ 15:10 & $78-93-3$ \\
\hline ND ug/L & 1.0 & 1 & $12 / 08 / 0815: 10$ & $104-51-8$ \\
\hline ND ug/L & 1.0 & 1 & $12 / 08 / 0815: 10$ & $135-98-8$ \\
\hline ND ug/L & 1.0 & 1 & $12 / 08 / 0815: 10$ & $98-06-6$ \\
\hline ND ug/L & 5.0 & 1 & $12 / 08 / 0815: 10$ & $75-15-0$ \\
\hline ND ug/L & 1.0 & 1 & $12 / 08 / 0815: 10$ & $56-23-5$ \\
\hline ND ug/L & 1.0 & 1 & $12 / 08 / 0815: 10$ & $108-90-7$ \\
\hline ND ug/L. & 1.0 & 1 & $12 / 08 / 0815: 10$ & $75-00-3$ \\
\hline ND ug/L & 1.0 & 1 & $12 / 08 / 0815: 10$ & $67-66-3$ \\
\hline $1.1 \mathrm{ug} / \mathrm{L}$ & 1.0 & 1 & $12 / 08 / 0815: 10$ & $74-87-3$ \\
\hline ND ug/L & 1.0 & 1 & $12 / 08 / 0815: 10$ & $95-49-8$ \\
\hline ND ug/L & 1.0 & 1 & $12 / 08 / 0815: 10$ & $106-43-4$ \\
\hline ND ug/L & 2.5 & 1 & $12 / 08 / 0815: 10$ & $96-12-8$ \\
\hline ND ug/L. & 1.0 & 1 & $12 / 08 / 08$ 15:10 & $124-48-1$ \\
\hline ND ug/L & 1.0 & 1 & $12 / 08 / 08 \quad 15: 10$ & $106-93-4$ \\
\hline ND ug/L. & 1.0 & 1 & $12 / 08 / 0815: 10$ & $74-95-3$ \\
\hline ND ug/L & 1.0 & 1 & $12 / 08 / 08$ 15:10 & $95-50-1$ \\
\hline ND ug/L & 1.0 & 1 & $12 / 08 / 08$ 15:10 & $541-73-1$ \\
\hline ND ug/L & 1.0 & 1 & $12 / 08 / 0815: 10$ & $106-46-7$ \\
\hline ND ug/L & 1.0 & 1 & $12 / 08 / 08 \quad 15: 10$ & $75-71-8$ \\
\hline ND ug/L & 1.0 & 1 & $12 / 08 / 0815: 10$ & $75-34-3$ \\
\hline ND ug/L & 1.0 & 1 & $12 / 08 / 0815: 10$ & $107-06-2$ \\
\hline ND ug/L. & 1.0 & 1 & $12 / 08 / 0815: 10$ & $540-59-0$ \\
\hline ND ug/L & 1.0 & 1 & $12 / 08 / 08 \quad 15: 10$ & $75-35-4$ \\
\hline ND ug/L & 1.0 & 1 & $12 / 08 / 0815: 10$ & $156-59-2$ \\
\hline ND ug/L & 1.0 & 1 & $12 / 08 / 0815: 10$ & $156-60-5$ \\
\hline ND ug/L & $1: 0$ & 1 & $12 / 08 / 0815: 10$ & $78-87-5$ \\
\hline ND ug/L & 1.0 & 1 & $12 / 08 / 0815: 10$ & $142-28-9$ \\
\hline ND ug/L & 1.0 & 1 & $12 / 08 / 0815: 10$ & $594-20-7$ \\
\hline ND ug/L & 1.0 & 1 & $12 / 08 / 08$ 15:10 & $563-58-6$ \\
\hline ND ug/L & 1.0 & 1 & $12 / 08 / 0815: 10$ & $10061-01-5$ \\
\hline ND ug/L & 1.0 & 1 & $12 / 08 / 0815: 10$ & $10061-02-6$ \\
\hline ND ug/L & 1.0 & 1 & $12 / 08 / 08$ 15:10 & $100-41-4$ \\
\hline ND ug/L & 1.0 & 1 & $12 / 08 / 0815: 10$ & $87-68-3$ \\
\hline ND ug/L & 10.0 & 1 & $12 / 08 / 08 \quad 15: 10$ & $591-78-6$ \\
\hline ND ug/L & 1.0 & 1 & $12 / 08 / 0815: 10$ & $98-82-8$ \\
\hline
\end{tabular}

REPORT OF LABORATORY ANALYSIS

Page 5 of 14

This report shall not be reproduced, except in full,

without the written consent of Pace Analytical Services, Inc.. 


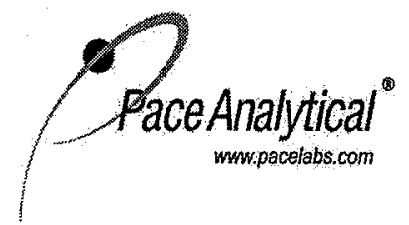

Pace Analytical Services, Inc. 9608 Loiret Blvd.

Lenexa, KS 66219

(913)599-5665

ANALYTICAL RESULTS

Project: Morrill

Pace Project No.: 6050656

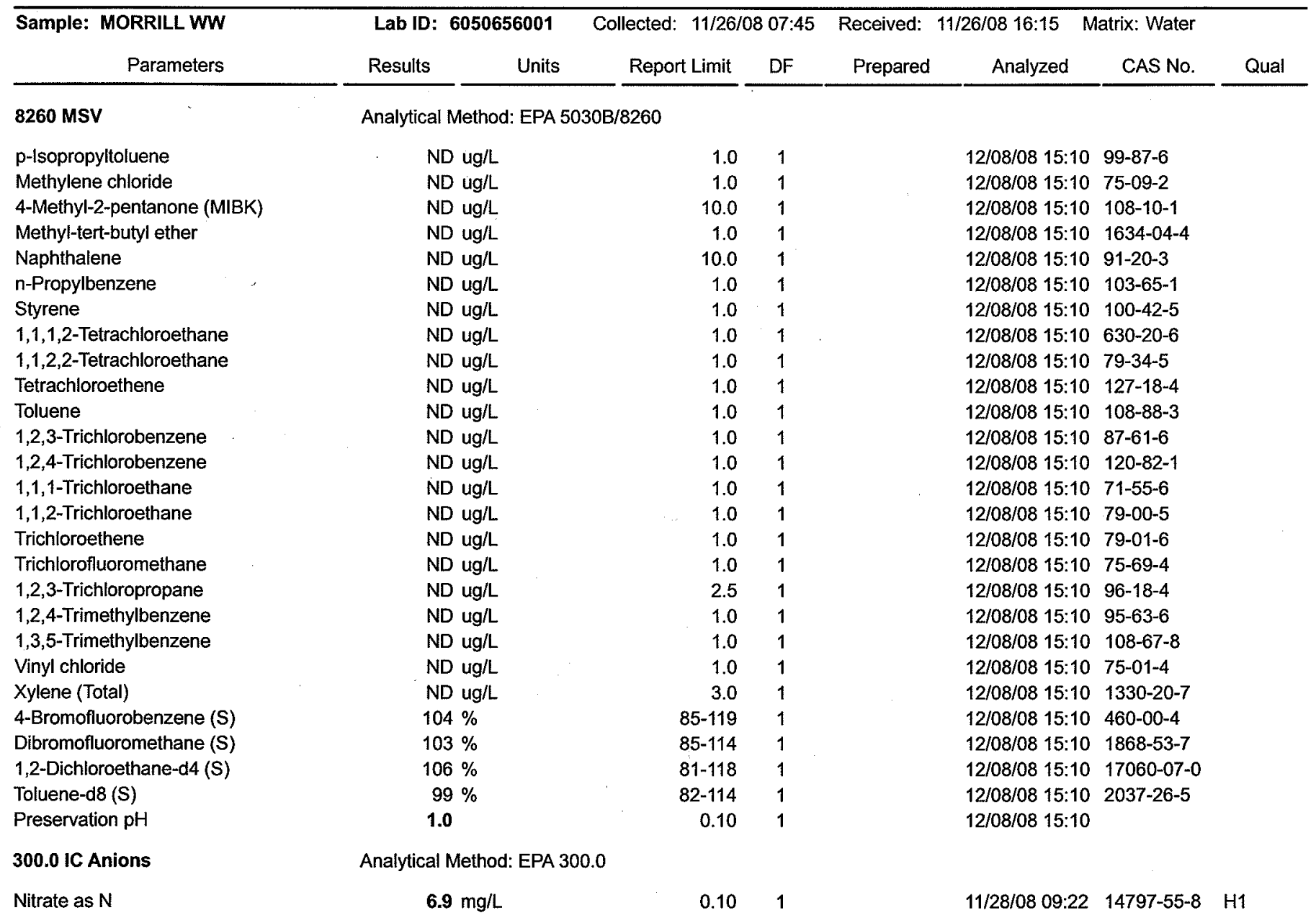

This report shall not be reproduced, except in full, without the written consent of Pace Analytical Services, Inc.. 


\section{QUALITY CONTROL DATA}

$\begin{array}{ll}\text { Project: } & \text { Morrill } \\ \text { Pace Project No:: } & 605065\end{array}$

\begin{tabular}{llll}
\hline QC Batch: & WETA18508 & Analysis Method: & EPA 300.0 \\
QC Batch Method: & EPA 300.0 & Analysis Description: & 300.0 IC Anions
\end{tabular}

Associated Lab Samples: 6050656001

METHOD BLANK: $414626 \quad$ Matrix: Water

Associated Lab Samples: 6050656001

\begin{tabular}{|c|c|c|c|c|c|}
\hline Parameter & Units & $\begin{array}{l}\text { Blank } \\
\text { Result }\end{array}$ & $\begin{array}{c}\text { Reporting } \\
\text { Limit }\end{array}$ & Analyzed & Qualifiers \\
\hline Nitrate as $\mathrm{N}$ & $\mathrm{mg} / \mathrm{L}$ & ND & 0.10 & $11 / 28 / 0808: 46$ & \\
\hline
\end{tabular}

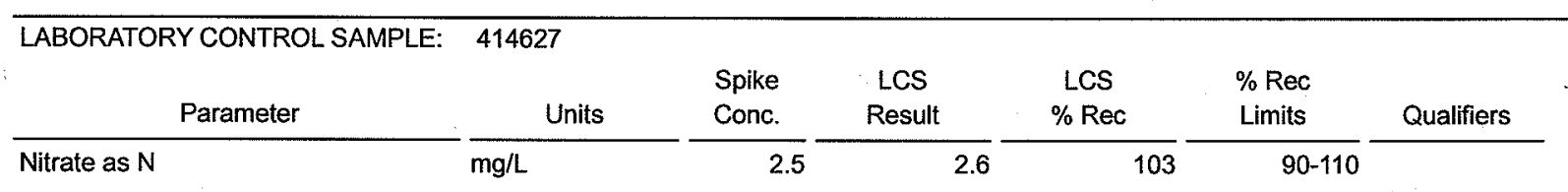

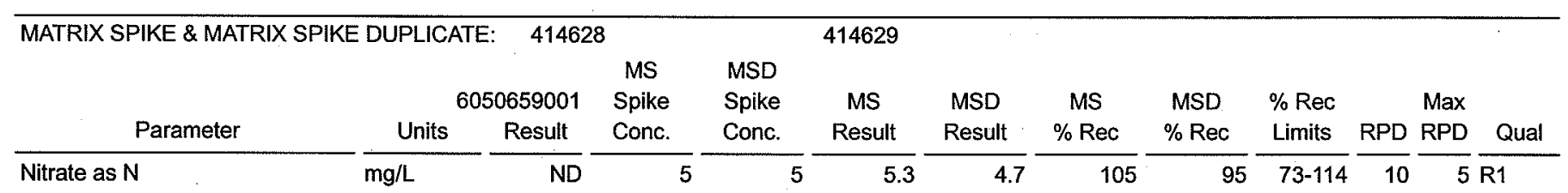




\section{QUALITY CONTROL DATA}

$\begin{array}{ll}\text { Project: } & \text { Morrill } \\ \text { Pace Project No.: } & 6050656\end{array}$

\begin{tabular}{llll}
\hline QC Batch: & OEXT/15115 & Analysis Method: & EPA 504.1 \\
QC Batch Method: & EPA 504.1 & Analysis Description: & GCS 504 EDB DBCP
\end{tabular}

Associated Lab Samples: 6050656001

METHOD BLANK: 418021

Matrix: Water

Associated Lab Samples: $\quad 6050656001$

\begin{tabular}{|c|c|c|c|c|c|}
\hline Parameter & Units & $\begin{array}{l}\text { Blank } \\
\text { Result }\end{array}$ & $\begin{array}{c}\text { Reporting } \\
\text { Limit }\end{array}$ & Analyzed & Qualifiers \\
\hline 1,2-Dibromoethane (EDB) & $\mathrm{ug} / \mathrm{L}$ & ND & 0.030 & $12 / 08 / 0814: 15$ & \\
\hline
\end{tabular}

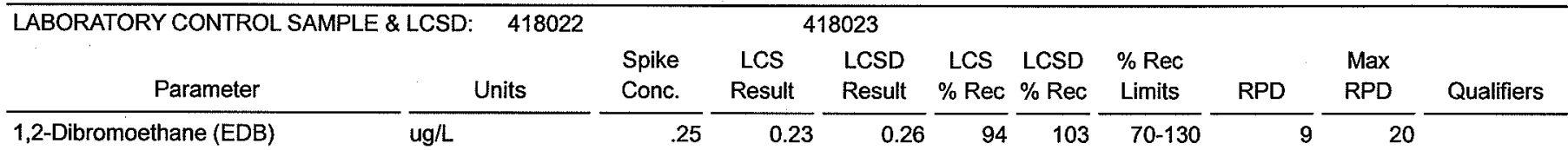

This report shall not be reproduced, except in full,

without the written consent of Pace Analytical Services, Inc.. 


\section{QUALITY CONTROL DATA}

Project: Morrill

Pace Project No.: 6050656

\begin{tabular}{llll}
\hline QC Batch: & MSV/18481 & Analysis Method: & EPA 5030B/8260 \\
QC Batch Method: & EPA 5030B/8260 & Analysis Description: & $8260 \mathrm{MSV}$ Water 10 mL Purge
\end{tabular}

Associated Lab Samples: 6050656001

METHOD BLANK: 418050

Associated Lab Samples: 6050656001

Parameter

1,1,1,2-Tetrachloroethane

1,1,1-Trichloroethane

1,1,2,2-Tetrachloroethane

1,1,2-Trichloroethane

1,1-Dichloroethane

1,1-Dichloroethene

1,1-Dichloropropene

1,2,3-Trichlorobenzene

1,2,3-Trichloropropane

1,2,4-Trichlorobenzene

1,2,4-Trimethylbenzene

1,2-Dibromo-3-chloropropane

1,2-Dibromoethane (EDB)

1,2-Dichlorobenzene

1,2-Dichloroethane

1,2-Dichloroethene (Total)

1,2-Dichloropropane

1,3,5-Trimethylbenzene

1,3-Dichlorobenzene

1,3-Dichloropropane

1,4-Dichlorobenzene

2,2-Dichloropropane

2-Butanone (MEK)

2-Chlorotoluene

2-Hexanone

4-Chlorotoluene

4-Methyl-2-pentanone (MIBK)

Acetone

Benzene

Bromobenzene

Bromochloromethane

Bromodichloromethane

Bromoform

Bromomethane

Carbon disulfide

Carbon tetrachloride

Chlorobenzene

Chloroethane

Chloroform

Chloromethane

cis-1,2-Dichloroethene

cis-1,3-Dichloropropene

Dibromochloromethane

Date: $12 / 10 / 2008$ 02:58 PM
Matrix: Water

\begin{tabular}{|c|c|c|c|c|}
\hline Units & $\begin{array}{l}\text { Blank } \\
\text { Result }\end{array}$ & $\begin{array}{l}\text { Reporting } \\
\text { Limit }\end{array}$ & Analyzed & Qualifiers \\
\hline ug/L & ND & 1.0 & $12 / 08 / 0811: 13$ & \\
\hline$u g / L$ & ND & 1.0 & $12 / 08 / 0811: 13$ & \\
\hline $\mathrm{ug} / \mathrm{L}$ & ND & 1.0 & $12 / 08 / 08$ 11:13 & \\
\hline$u g / L$ & $\mathrm{ND}$ & 1.0 & $12 / 08 / 0811: 13$ & \\
\hline
\end{tabular}

$\begin{array}{lll}\text { ND } & 1.0 & 12 / 08 / 08 \\ \text { ND } & 11: 13\end{array}$

$1.0 \quad 12 / 08 / 08$ 11:13

$1.0 \quad 12 / 08 / 08$ 11:13

$1.0 \quad 12 / 08 / 08 \quad 11: 13$

$1.0 \quad 12 / 08 / 08$ 11:13

$2.5 \quad 12 / 08 / 08$ 11:13

$1.0 \quad 12 / 08 / 08$ 11:13

$1.0 \quad 12 / 08 / 08$ 11:13

$2.512 / 08 / 08$ 11:13

$1.0 \quad 12 / 08 / 08$ 11:13

$1.0 \quad 12 / 08 / 08$ 11:13

$1.0 \quad 12 / 08 / 0811: 13$

$1.0 \quad 12 / 08 / 0811: 13$

$1.0 \quad 12 / 08 / 08$ 11:13

$1.0 \quad 12 / 08 / 0811: 13$

$1.0 \quad 12 / 08 / 0811: 13$

$1.0 \quad 12 / 08 / 0811: 13$

$1.0 \quad 12 / 08 / 0811: 13$

$1.0 \quad 12 / 08 / 0811: 13$

$10.0 \quad 12 / 08 / 0811: 13$

$1.0 \quad 12 / 08 / 0811: 13$

$10.0 \quad 12 / 08 / 0811: 13$

$1.0 \quad 12 / 08 / 08 \quad 11: 13$

$10.0 \quad 12 / 08 / 0811: 13$

$10.0 \quad 12 / 08 / 0811: 13$

$1.0 \quad 12 / 08 / 08 \quad 11: 13$

$1.0 \quad 12 / 08 / 0811: 13$

$1.0 \quad 12 / 08 / 08$ 11:13

$1.0 \quad 12 / 08 / 08$ 11:13

$1.0 \quad .12 / 08 / 08$ 11:13

$1.0 \quad 12 / 08 / 08$ 11:13

$5.0 \quad 12 / 08 / 0811: 13$

$1.0 \quad 12 / 08 / 0811: 13$

$1.0 \quad 12 / 08 / 08$ 11:13

$1.0 \quad 12 / 08 / 0811: 13$

$1.0 \quad 12 / 08 / 0811: 13$

$1.0 \quad 12 / 08 / 08$ 11:13

$1.0 \quad 12 / 08 / 08 \quad 11: 13$

$1.0 \quad 12 / 08 / 0811: 13$

$1.0 \quad 12 / 08 / 08$ 11:13

\section{REPORT OF LABORATORY ANALYSIS}

This report shall not be reproduced, except in full, without the written consent of Pace Analytical Services, Inc.. 


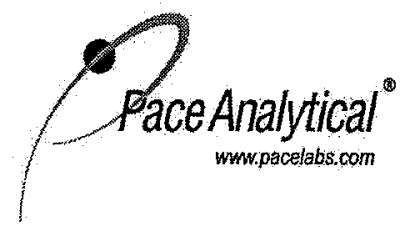

Pace Analytical Services, Inc. 9608 Loiret Bivd. Lenexa, KS 66219 (913)599-5665

\section{QUALITY CONTROL DATA}

Project: Morrill

Pace Project No.: 6050656

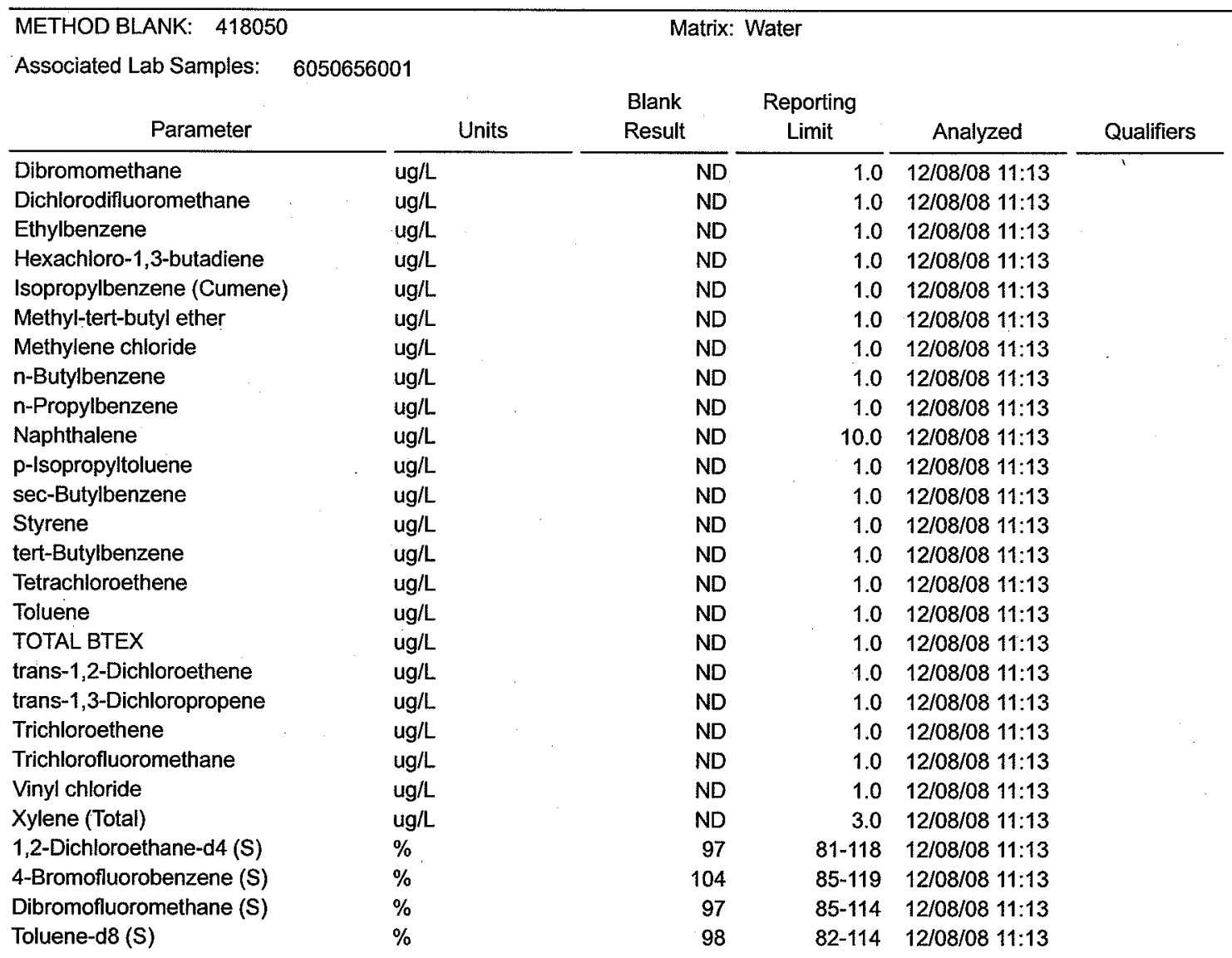

\begin{tabular}{|c|c|c|c|c|c|c|}
\hline \multirow{2}{*}{$\begin{array}{c}\text { LABORATORY CONTROL SAMPLE: } \\
\text { Parameter }\end{array}$} & \multicolumn{6}{|l|}{418051} \\
\hline & Units & $\begin{array}{l}\text { Spike } \\
\text { Conc. }\end{array}$ & $\begin{array}{l}\text { LCS } \\
\text { Result }\end{array}$ & $\begin{array}{c}\text { LCS } \\
\% \operatorname{ReC}\end{array}$ & $\begin{array}{l}\% \text { Rec } \\
\text { Limits }\end{array}$ & Qualifiers \\
\hline 1,1,1,2-Tetrachloroethane & $\mathrm{ug} / \mathrm{L}$ & 10 & 10.9 & 109 & $77-127$ & \\
\hline 1,1,1-Trichloroethane & $\mathrm{ug} / \mathrm{L}$ & 10 & 10.6 & 106 & $78-130$ & \\
\hline 1,1,2,2-Tetrachloroethane & $\mathrm{ug} / \mathrm{L}$ & 10 & 10.2 & 102 & $73-131$ & \\
\hline 1,1,2-Trichloroethane & $\mathrm{ug} / \mathrm{L}$ & 10 & 11.2 & 112 & $85-126$ & \\
\hline 1,1-Dichloroethane & $\mathrm{ug} / \mathrm{L}$ & 10 & 10.7 & 107 & $76-124$ & \\
\hline 1,1-Dichloroethene & $\mathrm{ug} / \mathrm{L}$ & 10 & 10.8 & 108 & $76-129$ & \\
\hline 1,1-Dichloropropene & $\mathrm{ug} / \mathrm{L}$ & 10 & 11.1 & 111 & $83-125$ & \\
\hline 1,2,3-Trichlorobenzene & ug/L & 10 & 11.6 & 116 & $78-129$ & \\
\hline 1,2,3-Trichloropropane & $\mathrm{ug} / \mathrm{L}$ & 10 & 11.0 & 110 & $69-117$ & \\
\hline 1,2,4-Trichlorobenzene & $\mathrm{ug} / \mathrm{L}$ & 10 & 11.6 & 116 & $79-127$ & \\
\hline 1,2,4-Trimethylbenzene & $\mathrm{ug} / \mathrm{L}$ & 10 & 10.3 & 103 & $82-124$ & \\
\hline 1,2-Dibromo-3-chloropropane & $\mathrm{ug} / \mathrm{L}$ & 10 & 13.0 & 130 & $62-141$ & \\
\hline 1,2-Dibromoethane (EDB) & $\mathrm{ug} / \mathrm{L}$ & 10 & 10.9 & 109 & $85-124$ & \\
\hline 1,2-Dichlorobenzene & $\mathrm{ug} / \mathrm{L}$ & 10 & 9.2 & 92 & $85-123$ & \\
\hline
\end{tabular}

Date: $12 / 10 / 2008$ 02:58 PM

This report shall not be reproduced, except in full, without the written consent of Pace Analytical Services, Inc.. 


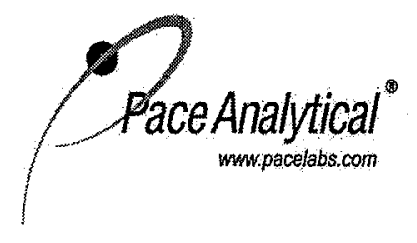

Pace Analytical Services, Inc. 9608 Loiret Blvd.

Lenexa, KS 66219

(913)599-5665

QUALITY CONTROL DATA

Project: Morrill

Pace Project No.: 6050656

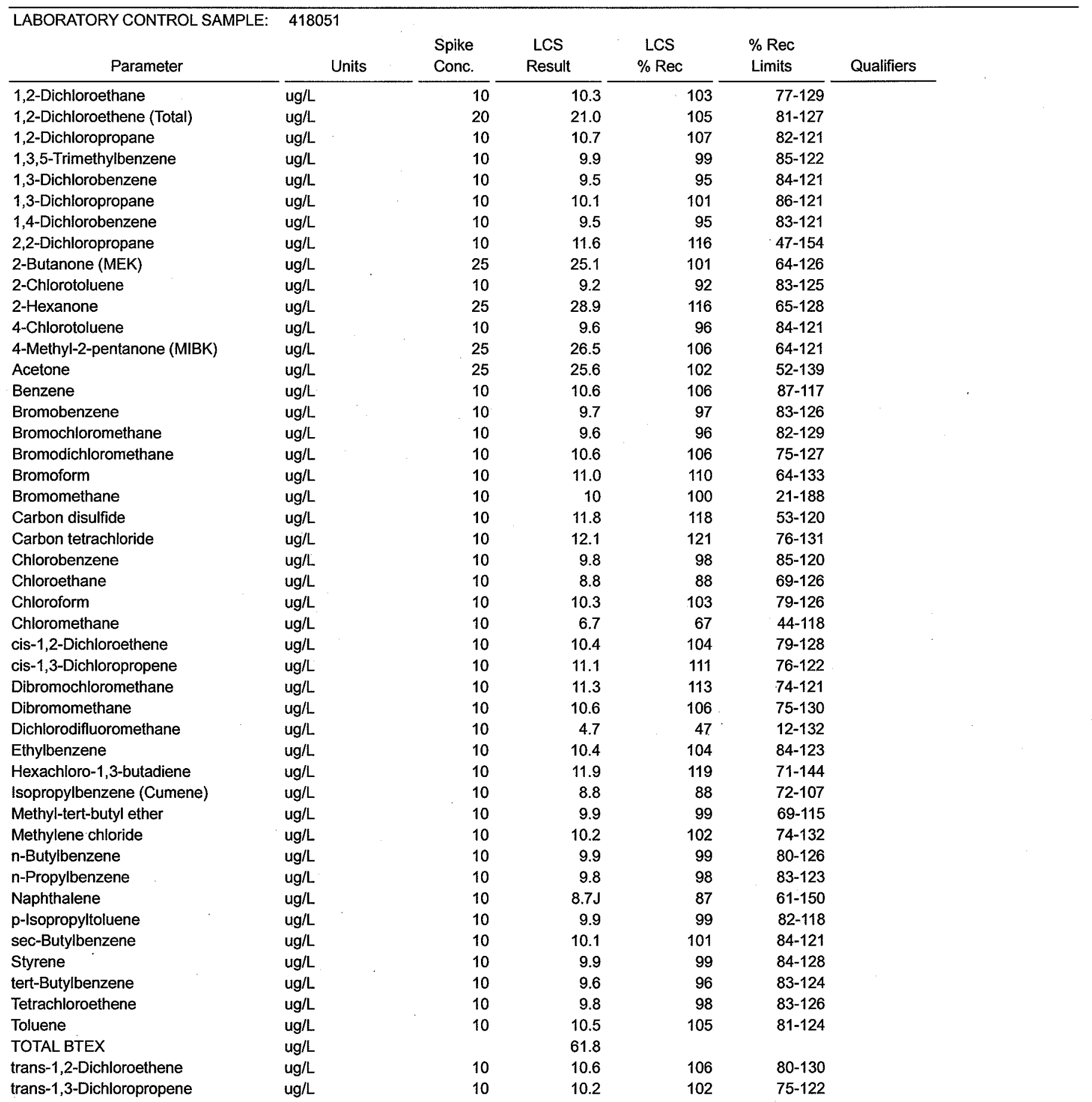

Date: $12 / 10 / 2008$ 02:58 PM

REPORT OF LABORATORY ANALYSIS

Page 11 of 14

This report shall not be reproduced, except in full,

without the written consent of Pace Analytical Services, Inc.. 


\section{QUALITY CONTROL DATA}

$\begin{array}{ll}\text { Project: } & \text { Morrill } \\ \text { Pace Project No.: } & 6050656\end{array}$

LABORATORY CONTROL SAMPLE: 418051

Parameter

Trichloroethene

Trichlorofluoromethane

Vinyl chloride

Xylene (Total)

1,2-Dichloroethane-d4 (S)

4-Bromofluorobenzene (S)

Dibromofluoromethane (S)

Toluene-d8 (S)

\begin{tabular}{|c|c|c|c|c|c|}
\hline Units & $\begin{array}{l}\text { Spike } \\
\text { Conc. }\end{array}$ & $\begin{array}{c}\text { LCS } \\
\text { Result }\end{array}$ & $\begin{array}{c}\text { LCS } \\
\% \operatorname{ReC}\end{array}$ & $\begin{array}{l}\% \text { Rec } \\
\text { Limits }\end{array}$ & Qualifiers \\
\hline $\mathrm{ug} / \mathrm{L}$ & 10 & 10.6 & 106 & $80-130$ & \\
\hline $\mathrm{ug} / \mathrm{L}$ & 10 & 9.7 & 97 & $65-113$ & \\
\hline $\mathrm{ug} / \mathrm{L}$ & 10 & 9.7 & 97 & $59-124$ & \\
\hline $\mathrm{ug} / \mathrm{L}$ & 30 & 30.3 & 101 & $83-125$ & \\
\hline$\%$ & & & 106 & $81-118$ & \\
\hline$\%$ & & & 102 & $85-119$ & \\
\hline$\%$ & & & 101 & $85-114$ & \\
\hline$\%$ & & & 101 & $82-114$ & \\
\hline
\end{tabular}




\section{QUALIFIERS}

$\begin{array}{ll}\text { Project: } & \text { Morrill } \\ \text { Pace Project No } . & 6050656\end{array}$

\section{DEFINITIONS}

DF - Dilution Factor, if reported, represents the factor applied to the reported data due to changes in sample preparation, dilution of the sample aliquot, or moisture content.

ND - Not Detected at or above adjusted reporting limit.

$\mathrm{J}$ - Estimated concentration above the adjusted method detection limit and below the adjusted reporting limit.

MDL - Adjusted Method Detection Limit.

S - Surrogate

1,2-Diphenylhydrazine ( 8270 listed analyte) decomposes to Azobenzene.

Consistent with EPA guidelines, unrounded data are displayed and have been used to calculate \% recovery and RPD values.

LCS(D) - Laboratory Control Sample (Duplicate)

MS(D) - Matrix Spike (Duplicate)

DUP - Sample Duplicate

RPD - Relative Percent Difference

Pace Analytical is NELAP accredited. Contact your Pace PM for the current list of accredited analytes.

\section{BATCH QUALIFIERS}

Batch: MSV/18481

[1]

A matrix spike/matrix spike duplicate was not performed for this batch due to insufficient sample volume.

\section{ANALYTE QUALIFIERS}

H1 Analysis conducted outside the EPA method holding time.

R1 RPD value was outside control limits. 
QUALITY CONTROL DATA CROSS REFERENCE TABLE

\begin{tabular}{ll} 
Project: & Morrill \\
Pace Project No.: & 6050656 \\
\hline
\end{tabular}

\begin{tabular}{lllll}
\hline Lab ID & Sample ID & QC Batch Method & QC Batch & $\frac{\text { Analytical Method }}{\text { WETA/8508 }}$ \\
\hline $\mathbf{6 0 5 0 6 5 6 0 0 1}$ & MORRILL WW & EPA 300.0 & OEXT/15115 EPA 504.1 \\
$\mathbf{6 0 5 0 6 5 6 0 0 1}$ & MORRILL WW & EPA 504.1 & Analytical \\
Batch
\end{tabular}




\section{Appendix C:}

Data Summary for Verification VOCs Analyses by TestAmerica 


\section{TestAmerica}

THE LEADER IN ENVIRONMENTAL TESTING

November 6, 2008

TestAmerica Laboratories, Inc.

Mr. Clyde Dennis

Argonne National Laboratory

9700 S. Cass Avenue

Building 203, Office B149

Argonne, IL 60439

Re: Laboratory Project No. 21005

Case: MORRILL; SDG: 128326

Dear Mr. Dennis:

Enclosed are analytical results for samples that were received by TestAmerica Burlington on October $22^{\text {nd }}, 2008$. Laboratory identification numbers were assigned, and designated as follows:

\begin{tabular}{|c|c|}
\hline 10 & $\begin{array}{l}\text { Client } \\
\text { Sample ID }\end{array}$ \\
\hline
\end{tabular}

Received: 10/22/08 ETR No: 128326

$\begin{array}{llll}772511 & \text { MRMW1S-W-27620 } & 10 / 20 / 08 & \text { WATER } \\ 772512 & \text { MRMW6S-W-27626 } & 10 / 20 / 08 & \text { WATER } \\ 772513 & \text { MRMW11S-W-27631 } & 10 / 20 / 08 & \text { WATER } \\ 772514 & \text { MRMW2S-W-27622 } & 10 / 21 / 08 & \text { WATER } \\ 772515 & \text { MRMW3S-W-27623 } & 10 / 21 / 08 & \text { WATER } \\ 772516 & \text { MRMW1S-W-27649 } & 10 / 21 / 08 & \text { WATER } \\ 772517 & \text { MRMW3S-W-27650 } & 10 / 21 / 08 & \text { WATER } \\ 772518 & \text { MRMW11S-W-27651 } & 10 / 21 / 08 & \text {-WATER } \\ 772519 & \text { MRMW2S-W-27652 } & 10 / 21 / 08 & \text { WATER } \\ 772520 & \text { MRQCTB-W-27638 } & 10 / 21 / 08 & \text { WATER } \\ 772521 & \text { VHBLK01 } & 10 / 22 / 08 & \text { WATER }\end{array}$

Documentation of the condition of the samples at the time of their receipt and any exception to the laboratory's Sample Acceptance Policy is documented in the Sample Handling section of this submittal. The samples, as received, were not acid preserved. On that basis the laboratory did provide for the analytical work to be performed within seven days of sample collection.

In order to accommodate field length limitations in processing the data summary forms, the laboratory did, in certain instances, abbreviate the sample identifier. The electronically formatted data provides for the full sample identifier. 


\section{SOM01.2 Volatile Organics (Trace Level Water)}

A storage blank was prepared for volatile organics analysis, and stored in association with the storage of the sample. That storage blank, identified as VHBLK01, was carried through the holding period with the samples, and analyzed.

Samples MRMW3S-W-27650 and MRMW11S-W-27651 were analyzed at a dilution, based on the results of preliminary screening: An additional, more concentrated analysis was performed on each sample in order to provide a lower reporting limit for those target analytes that were not identified as constituents in the primary analysis. An additional, dilution analysis was performed on samples MRMW11S-W-27631, MRMW3S-W-27623, and MRMW1S-W-27649 in order to provide quantification within the range of calibrated instrument response. Both sets of results for the analysis of samples MRMW1 is-W-27631, MRMW3S-W-27623, MRMW1S-W-27649, MRMW3S-W-27650, and MRMW11S-W-27651 are included in this submittal. Each of the analyses associated with the sample set exhibited an acceptable internal standard performance. There was an acceptable recovery of each deuterated monitoring compound (DMC) in the analysis of each method blank and each instrument blank associated with the analytical work, and in the analysis of the storage blank associated with the sample set. The analysis of the samples in this sample set did meet the technical acceptance criteria specific to DMC recoveries, although not all DMC recoveries were within the control range in each analysis. The technical acceptance criteria does provide for the recovery of up to three DMCs to fall outside of the control range in the analysis of field samples. The recoveries of 2 -butanone- $d_{5}$ and 2 -hexanone- $d_{5}$ were elevated in the analysis of several of the field samples. Matrix spike and matrix spike duplicate analyses were not performed on samples in this sample set. Trace concentrations of carbon disulfide and 1,2,3-trichlorobenzene were identified in the analysis of one of the method blanks associated with the analytical work. The concentration of each compound in that analysis was below the established reporting limit, and the analysis did meet the technical acceptance criteria for a compliant method blank analysis. The analysis of the second method blank associated with the analytical work was free of target analyte contamination, as was the analysis of each instrument blank and the analysis of the storage blank. Present in the method blank and storage blank analyses was a non-target constituent that represents a compound that is related to the DMC formulation. The fact that the presence of this compound is not within the laboratory's. control is at issue. The derived results for that compound have been qualified with an " $X$ " qualifier to reflect the source of the contamination.

The responses for each target analyte met the relative standard deviation criterion in each initial calibration. The response for each target analyte met the percent difference criterion in the continuing calibration check acquisition. The response for each target analyte met the 50.0 percent difference criterion in the closing calibration check acquisition.

The primary quantitation mass for methylcyclohexane that is specified in the Statement of Work is mass 83 . The laboratory did identify a contribution to mass 83 from 1,2-dichloropropane-d $d_{6}$, one of the deuterated monitoring compounds (DMCs). The laboratory did change the primary quantitation mass assignment to mass 55 for the quantification of methylcyciohexane:

Mañual integration was employed in deriving certain of the analytical results. The values that have been derived from manual integration are qualified on the quantitation reports. Extracted ion current profiles for each manual integration are included in the data package, and further 


\section{TestAmerica}

THE LEADER IN ENVIRONMENTAL TESTING

documented in the Sample Preparation section of this submittal.

Any reference within this report to Severn Trent Laboratories, Inc. or STL, should be understood to refer to TestAmerica Laboratories, Inc. (formerly known as Severn Trent Laboratories, Inc.) The analytical results associated with the samples presented in this test report were generated under a quality system that adheres to requirements specified in the NELAC standard. Release of the data in this test report and any associated electronic deliverables is authorized by the Laboratory Director's designee as verified by the following signature.

If there are any questions regarding this submittal, please contact me at 802 660-1990.

Sincerely,

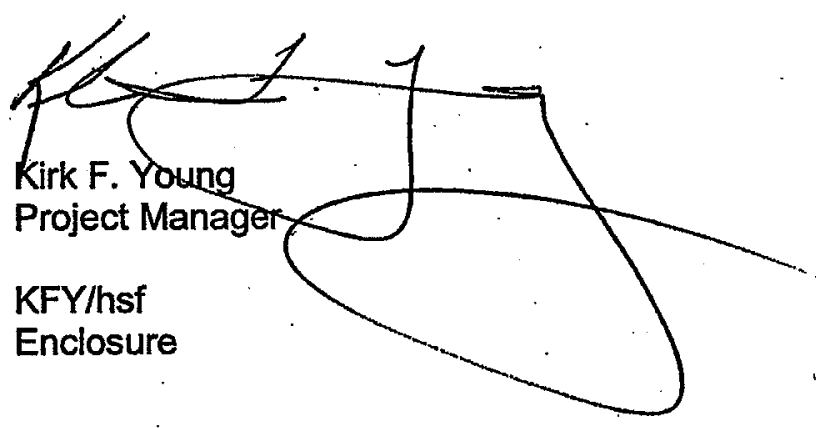




\section{Organic}

U: Compound analyzed but not detected at a concentration above the reporting limit.

$\mathrm{J}: \quad$ Estimated value.

N: Indicates presumptive evidence of a compound. This flag is used only for tentatively identified compounds (TICs) where the identification of a compound is based on a mass spectral library search.

P: $\quad$ SW-846: The relative percent difference for detected concentrations between two GC columns is greater than $40 \%$. Unless otherwise specified the higher of the two values is reported on the Form I.

CLP SOW: Greater than $25 \%$ difference for detected concentrations between two GC columns. Unless otherwise specified the lower of the two values is reported on the Form I.

C: Pesticide result whose identification has been confirmed by GC/MS.

B: Analyte is found in the sample and the associated method blank. The flag is used for tentatively identified compounds as well as positively identified compounds.

E: Compounds whose concentrations exceed the upper limit of the calibration range of the instrument for that specific analysis.

D: Concentrations identified from analysis of the sample at a secondary dilution.

A: Tentatively identified compound is a suspected aldol condensation product.

$X, Y, Z$ : Laboratory defined flags that may be used alone or combined, as needed. If used, the description of the flag is defined in the project narrative.

\section{Inorganic/Metals}

E: $\quad$ Reported value is estimated due to the presence of interference.

N: Matrix spike sample recovery is not within control limits.

* Duplicate sample analysis is not within control limits.

B: The result reported is less than the reporting limit but greater than the instrument detection limit.

U: Analyte was analyzed for but not detected above the reporting limit.

\section{Method Codes:}

$P \quad$ ICP-AES

MS ICP-MS

CV Cold Vapor AA

AS Semi-Automated Spectrophotometric 


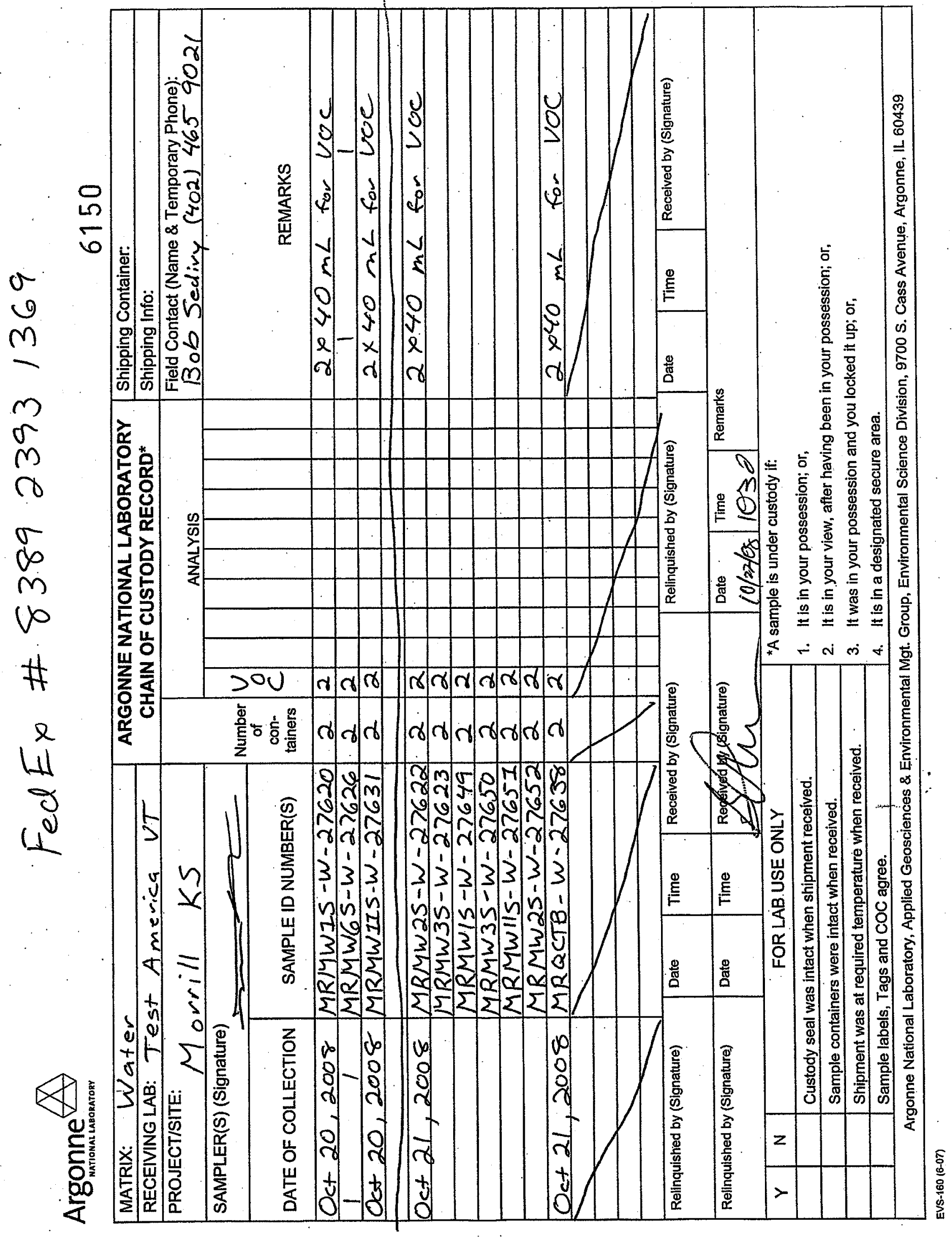




\section{TestAmerica

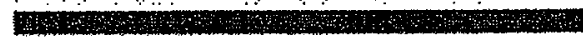 \\ THE LEADER IN ENVIRONMENTTAL TESTING}

\section{Sample Data Summary - SOM01.2 Volatiles - Trace}


$I A$ - FORM I VOA-I

VOLATILE ORGANICS ANALYSIS DATA SHEET
EPA SAMPLE NO.

I1SW2763I
Lab Name: TESTAMERICA BURLINGTON

Lab Code: STLV Case No.: MORRILL

Matrix: (SOIL/SED/WATER) Water

Sample wt/vol: $25.0 \quad(\mathrm{~g} / \mathrm{mL}) \mathrm{mL}$

Level: (TRACE/LOW/MED) TRACE

\% Moisture: not dec.

GC Column: DB-624

ID $: 0.53$

Soil Extract Volume:

Purge Volume: 25.0
Contract: $8 \mathrm{E}-00302$

Mod. Ref No.:
Iab Sample ID: 772513

Lab File ID: 772513

Date Received: 10/22/2008

Date Analyzed: 10/23/2008

Dilution Factor: 1.0

Soil Aliquot Volume:

(uL)

\begin{tabular}{|c|c|c|c|}
\hline $\begin{array}{l}\text { CAS NO. } \\
========== \\
75-71-8 \\
74-87-3 \\
75-01-4 \\
74-83-9 \\
75-00-3 \\
75-69-4 \\
75-35-4 \\
76-13-1 \\
67-64-1 \\
75-15-0 \\
79-20-9 \\
75-09-2 \\
156-60-5 \\
1634-04-4 \\
75-34-3 \\
156-59-2 \\
78-93-3 \\
74-97-5 \\
67-66-3 \\
71-55-6 \\
110-82-7 \\
56-23-5 \\
71-43-2 \\
107-06-2\end{array}$ & 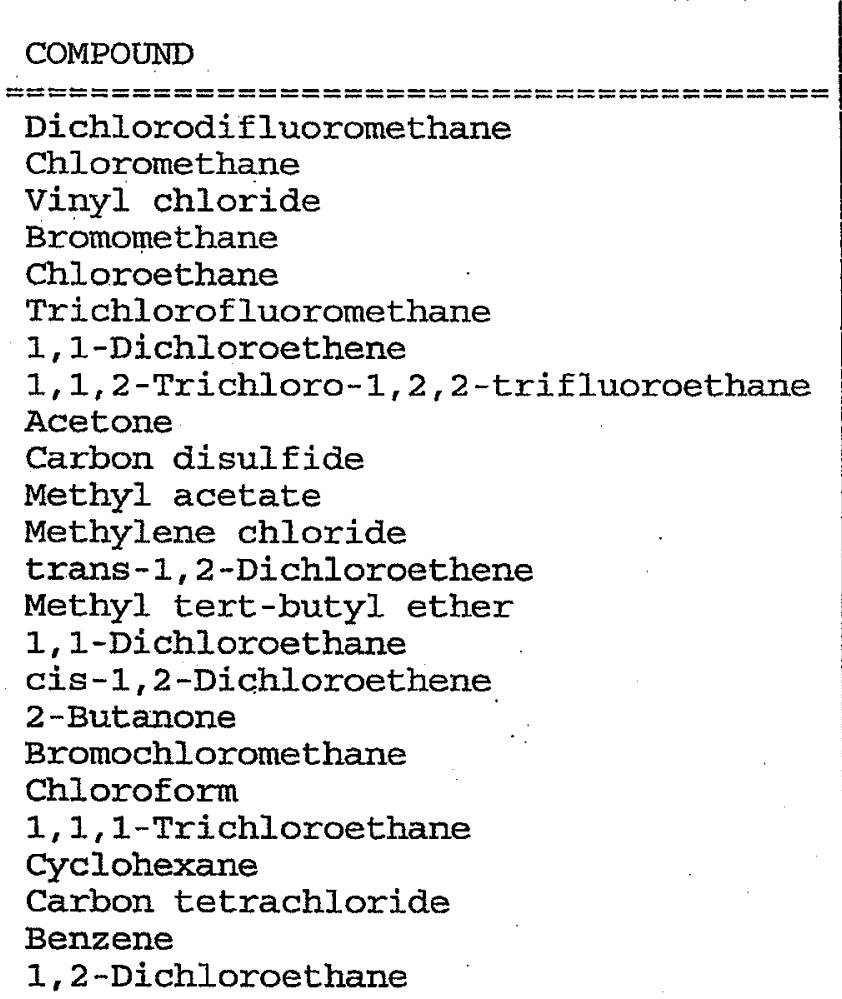 & 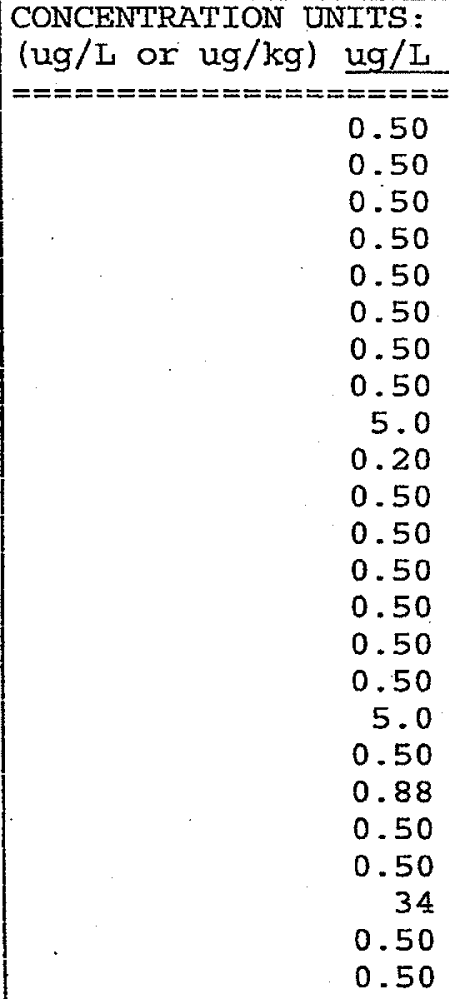 & $\begin{array}{l}======== \\
U \\
U \\
U \\
U \\
U \\
U \\
U \\
U \\
U \\
U B \\
U \\
U \\
U \\
U \\
U \\
U \\
U \\
U\end{array}$ \\
\hline
\end{tabular}

Report 1,4-Dioxane for Low-Medium VOA analysis only 
Lab Name: TESTAMERICA BURLINGTON

Lab. Code: STLV

Case NO.: MORRILL

Matrix: (SOIL/SED/WATER) Water

Sample wt/vol $=25.0(\mathrm{~g} / \mathrm{mI}) \mathrm{mL}$

Leve1: (TRACE/LOW/MED) TRACE

$\because$ Moisture: not dec.

GC Column: DB-624

ID : 0.53

Soil Extract Volume:

Purge volume: 25.0
Contract: 8E-00302

SDG No.: 128326

Mod. Ref No.:

Lab Sample ID: 772513

Lab File ID: 772513

Date Received: 10/22/2008

Date Analyzed: 10/23/2008

Dilution Factor: 1.0

(uI) Soil Aliquot volume:

(uL)

(mL)

\begin{tabular}{|c|c|c|c|}
\hline $\begin{array}{r}\text { CAS NO. } \\
=========== \\
79-01-6 \\
108-87-2 \\
78-87-5 \\
75-27-4 \\
10061-01-5 \\
108-10-1 \\
108-88-3 \\
10061-02-6 \\
79-00-5 \\
127-18-4 \\
591-78-6 \\
124-48-1 \\
106-93-4 \\
108-90-7 \\
100-41-4 \\
95-47-6 \\
179601-23-1 \\
100-42-5 \\
75-25-2 \\
98-82-8 \\
79-34-5 \\
541-73-1 \\
106-46-7 \\
95-50-1 \\
96-12-8 \\
120-82-1 \\
87-61-6\end{array}$ & 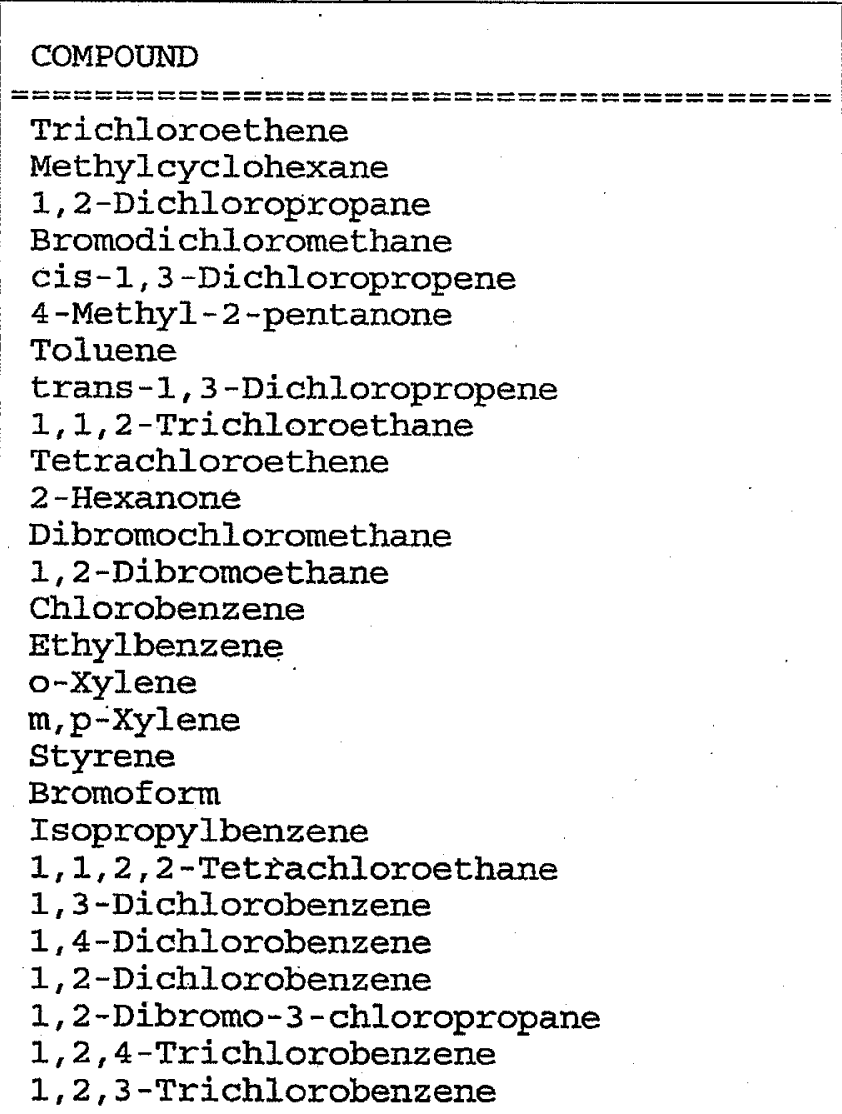 & 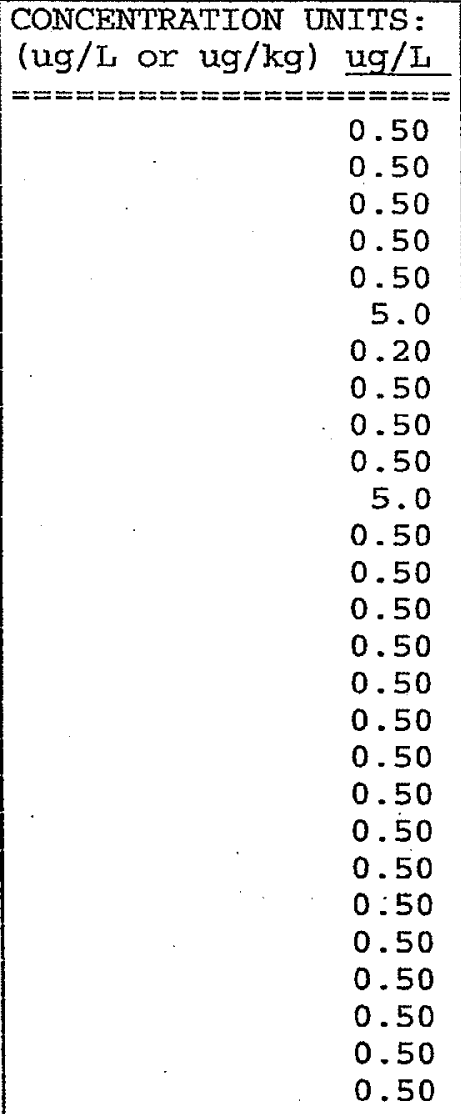 & $\begin{array}{l}\text { Q } \\
======= \\
U \\
U \\
U \\
U \\
U \\
U \\
J \\
U \\
U \\
U \\
U \\
U \\
U \\
U \\
U \\
U \\
U \\
U \\
U \\
U \\
U \\
U \\
U \\
U \\
U \\
U \\
U\end{array}$ \\
\hline
\end{tabular}


IA - FORM I VOA-1

VOLATILE ORGANICS ANAIYSIS DATA SHEET
EPA SAMPLE NO.

IISW2763IDL
Lab Name: TESTAMERICA BURLINGTON

Lab Code: STLV Case No.: MORRILI

Matrix: (SOII/SED/WATER) Water

Sample wt/vol: $25.0 \quad(\mathrm{~g} / \mathrm{mL}) \mathrm{mL}$

Leve1: (TRACE/LOW/MED) TRACE

$\%$ Moisture: not dec.

GC Column: DB-624

ID : 0.53

Soil Extract Volume:

Purge Volume: 25.0
Contract: $8 \mathrm{E}-00302$

Mod. Ref No.:

SDG NO.: 128326
Lab Sample ID: 772513D1

Lab File ID: $772513 D$

Date Received: 10/22/2008

Date Analyzed: 10/24/2008

(mm) Dilution Factor: 2.2

(uI) Soil Aliquot Volume:

(uL)

(mL)

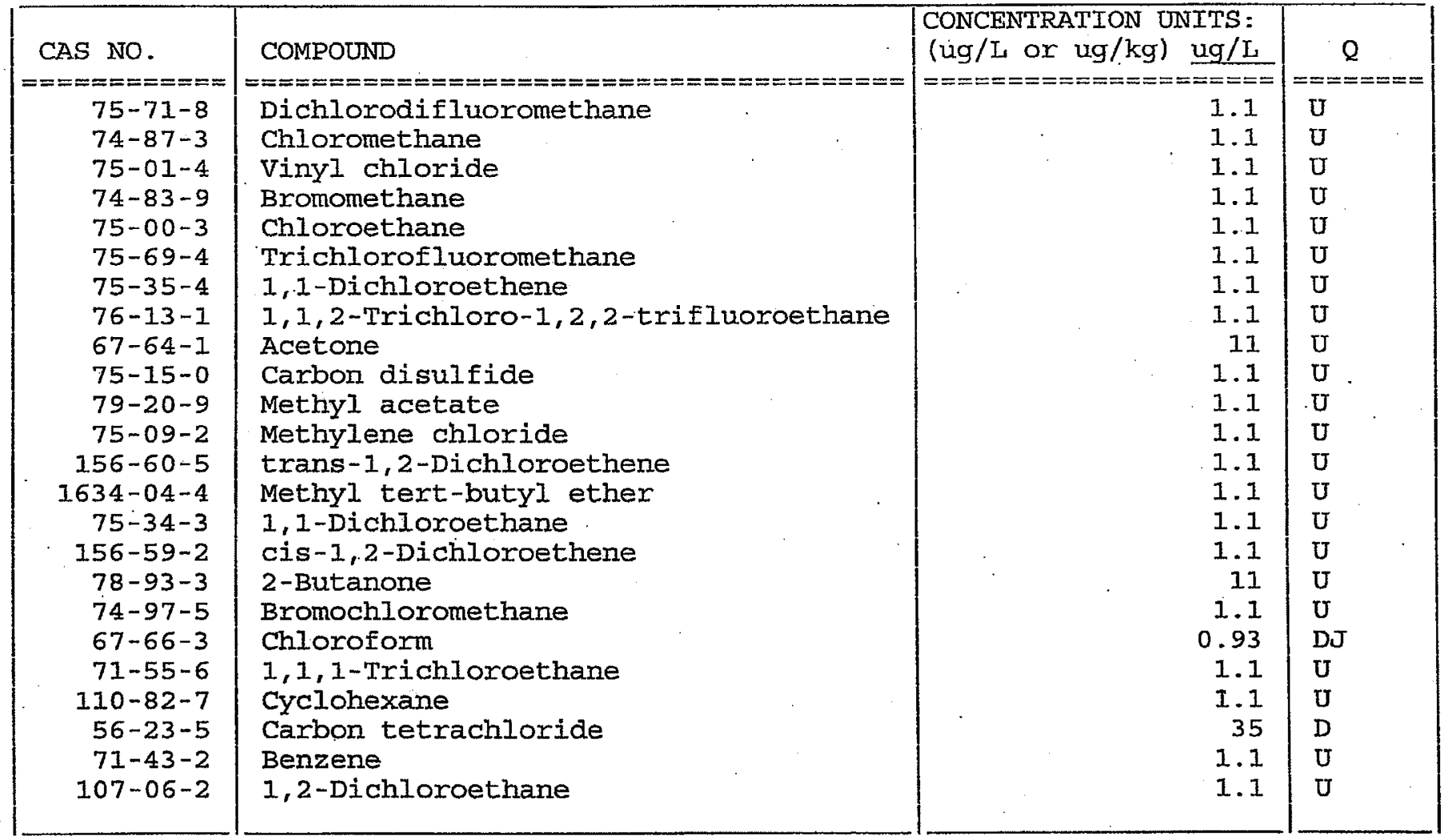

Report 1,4-Dioxane for Low-Medium VOA analysis only 
$1 B$ - FORM I VOA-2

VOLATILE ORGANICS ANALYSIS DATA SHEET

EPA SAMPLE NO.

11SW27631DL
Lab Name: TESTAMERICA BURLINGTON

Lab Code: STLV Case No.: MORRILI Matrix: (SOIL/SED/WATER) Water

Sample wt/vol: 25.0

$(\mathrm{g} / \mathrm{mL}) \mathrm{mL}$

Level: (TRACE/LOW/MED) TRACE

\% Moisture: not dec.

GC Column: $D B-624$

ID $: 0.53$

Soil Extract Volume:

Purge Volume: 25.0
Contract: $8 \mathrm{E}-00302$

SDG NO.: 128326

Mod. Ref No.:

Lab Sample ID: 772513DI

Lab File ID: $772513 \mathrm{D}$

Date Received: 10/22/2008

Date Analyzed: 10/24/2008

Dilution Factor: 2.2

(uL) Soil Aliquot Volume:

(uL)

(mL)

\begin{tabular}{|c|c|c|c|}
\hline $\begin{array}{r}\text { CAS NO. } \\
=========== \\
79-01-6 \\
108-87-2 \\
78-87-5 \\
75-27-4 \\
10061-01-5 \\
108-10-1 \\
108-88-3 \\
10061-02-6 \\
79-00-5 \\
127-18-4 \\
591-78-6 \\
124-48-1 \\
106-93-4 \\
108-90-7 \\
100-41-4 \\
95-47-6 \\
179601-23-1 \\
100-42-5 \\
75-25-2 \\
98-82-8 \\
79-34-5 \\
541-73-1 \\
106-46-7 \\
95-50-1 \\
96-12-8 \\
120-82-1 \\
87-61-6\end{array}$ & 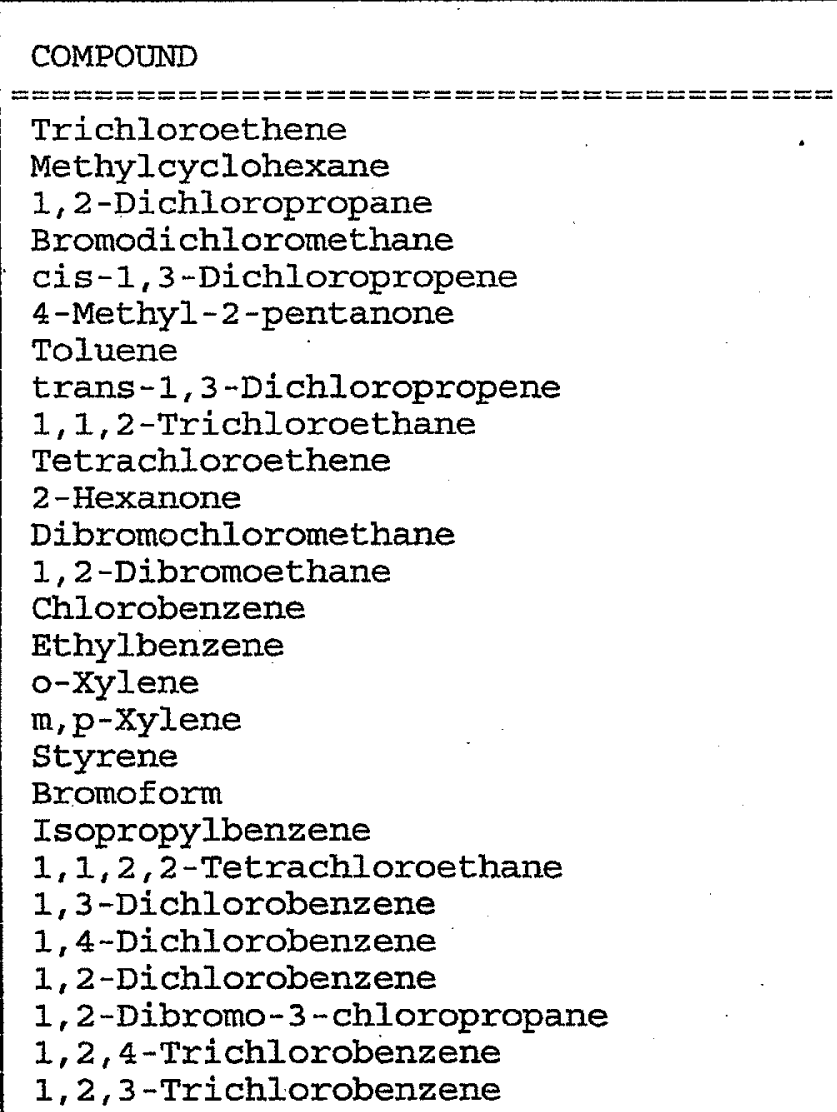 & 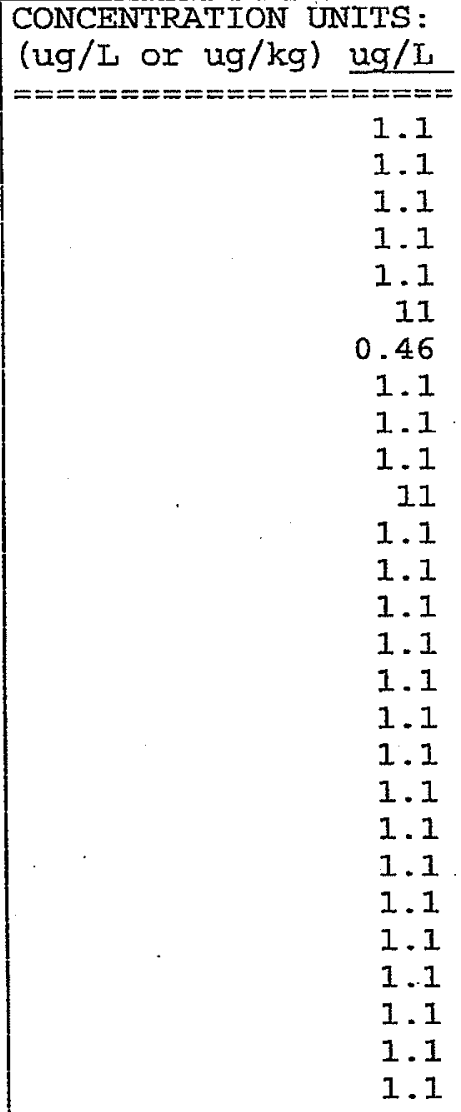 & $\begin{array}{l}========= \\
U \\
U \\
U \\
U \\
U \\
U \\
D J \\
U \\
U \\
U \\
U \\
U \\
U \\
U \\
U \\
U \\
U \\
U \\
U \\
U \\
U \\
U \\
U \\
U \\
U \\
U \\
U\end{array}$ \\
\hline
\end{tabular}


IA - FORM I VOA-I

VOLATILE ORGANICS ANALYSIS DATA SHEET

EPA SAMPIE NO.

Lab Name: TESTAMERICA BURLINGTON

Contract: $8 \mathrm{E}-00302$

Lab. Code: STLV Case No.: MORRILL Mod. Ref No.:

SDG No.: 128326

Matrix: (SOIL/SED/WATER) Water

Sample wt/vol: $25.0 \quad(\mathrm{~g} / \mathrm{mL}) \mathrm{mL}$

Level: (TRACE/LOW/MED) TRACE

\% Moisture: not dec.

GC Column: DB-624

ID $: 0.53$

Soil Extract Volume:

Purge Volume: 25.0
Lab Sample ID: 772518

Lab File ID: 772518

Date Received: $10 / 22 / 2008$

Date Analyzed: 10/24/2008

Dilution Factor: 1.0

(uI) Soil Aliquot Volume:

(uL)

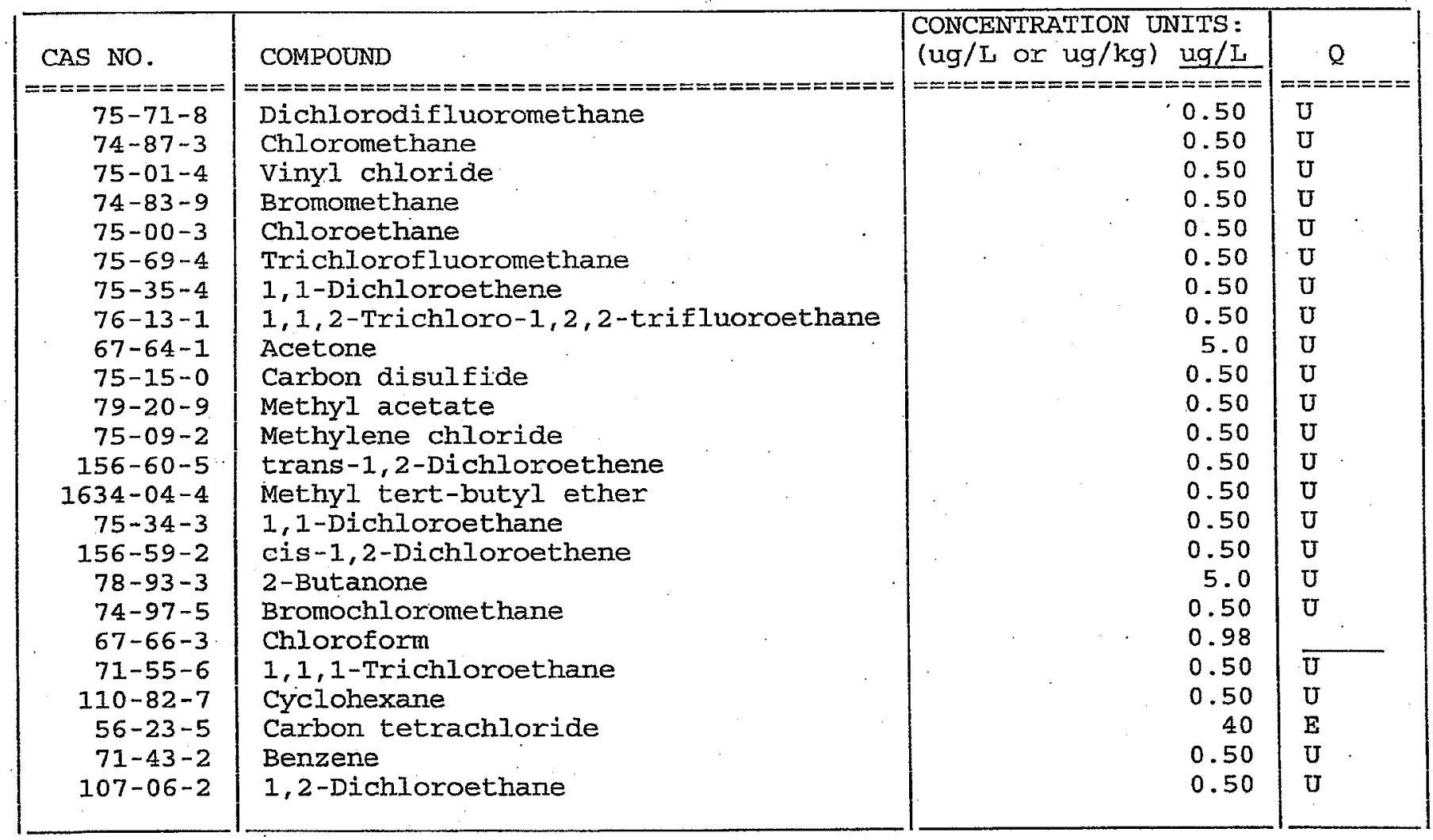

Report 1,4-Dioxane for Low-Medium VOA analysis only. 
$I B$ - FORM I VOA-2

VOLATIIE ORGANICS ANALYSIS DATA SHEET

EPA SAMPLE NO.

Lab Name: TESTAMERICA BURLINGTON

Contract: $8 \mathrm{E}-00302$

Lab Code: STLV Case No.: MORRILL Mod. Ref No.:

SDG NO.: 128326

Matrix: (SOIL/SED/WATER) Water

Sample wt/vol: $25.0(\mathrm{~g} / \mathrm{mL}) \mathrm{mL}$

Level : (TRACE/LOW/MED) TRACE

$\%$ Moisture: not dec.

GC Column: DB-624

ID $: 0.53 \quad(\mathrm{~mm})$

(UI)

Lab Sample ID: 772518

Lab File ID: 772518

Date Received: 10/22/2008

Date Analyzed: 10/24/2008

Soil Extract Volume:

(mL)

Purge volume: 25.0

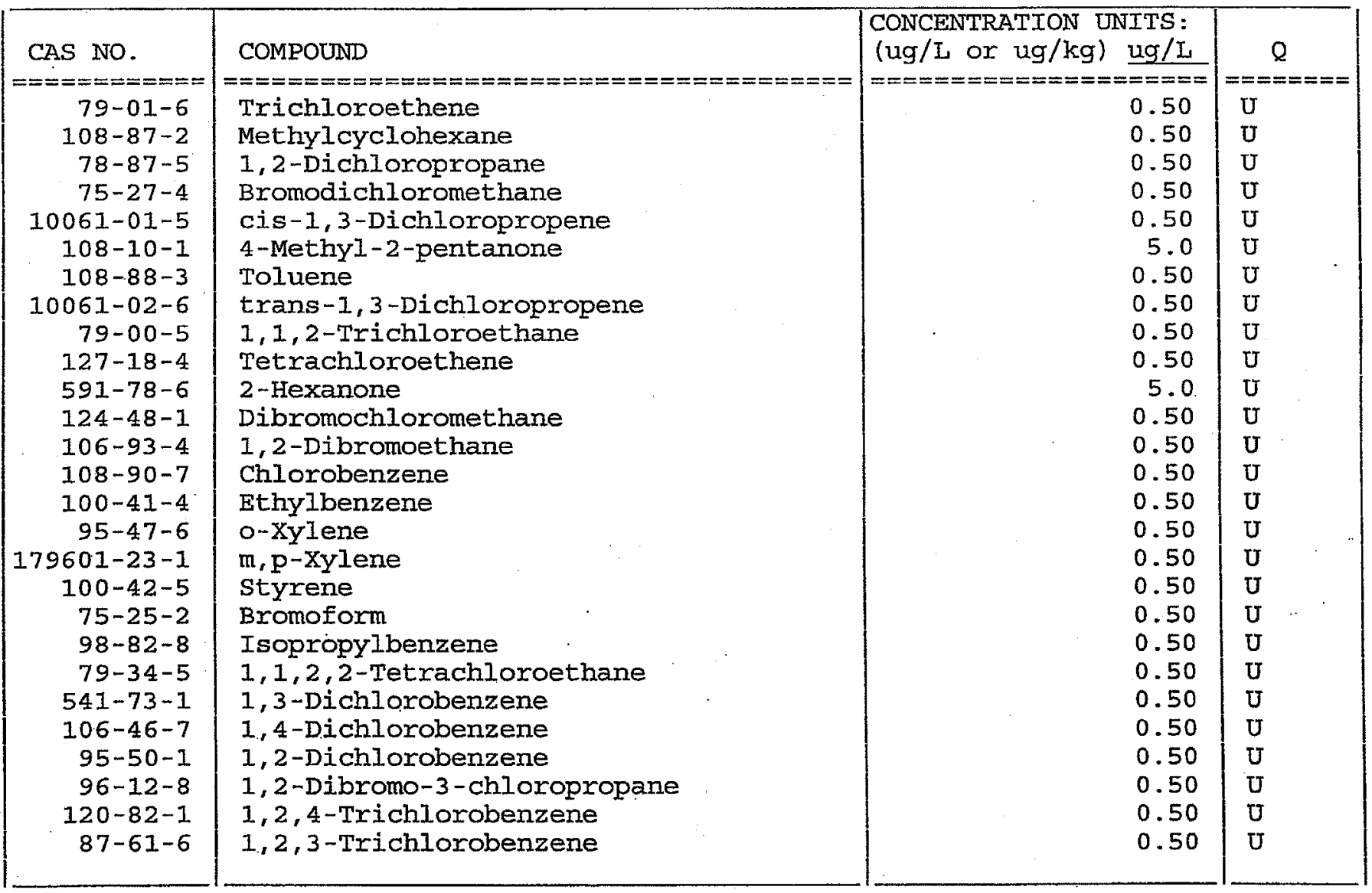


IA - FORM I VOA-1

VOLATILE ORGANICS ANALYSIS DATA SHEET

EPA SAMPLE NO.

11 SW27651DL

Lab Name: TESTAMERICA BURLINGTON

Contract: $8 \mathrm{E}-00302$

Lab Code: STLV Case No.: MORRILL Mod. Ref No.:

SDG NO.: 128326

Matrix: (SOTL/SED/WATER) Water

Sample wt/vol: 25.0 (g/mL) $\mathrm{mL}$

Level: (TRACE/LOW/MED) TRACE

$\because$ Moisture: not dec.

GC Column: DB-624

ID $: 0.53$

Soil Extract Volume:

Purge Volume: 25.0
Lab Sample ID: 772518D1

Lab File ID: 772518D

Date Received: 10/22/2008

Date Analyzed: 10/24/2008

Dilution Factor: 2.5

Soil Aliquot Volume:

(UI)

\begin{tabular}{|c|c|c|c|}
\hline $\begin{array}{c}\text { CAS NO. } \\
========== \\
75-71-8 \\
74-87-3 \\
75-01-4 \\
74-83-9 \\
75-00-3 \\
75-69-4 \\
75-35-4 \\
76-13-1 \\
67-64-1 \\
75-15-0 \\
79-20-9 \\
75-09-2 \\
156-60-5 \\
1634-04-4 \\
75-34-3 \\
156-59-2 \\
78-93-3 \\
74-97-5 \\
67-66-3 \\
71-55-6 \\
110-82-7 \\
56-23-5 \\
71-43-2 \\
107-06-2\end{array}$ & 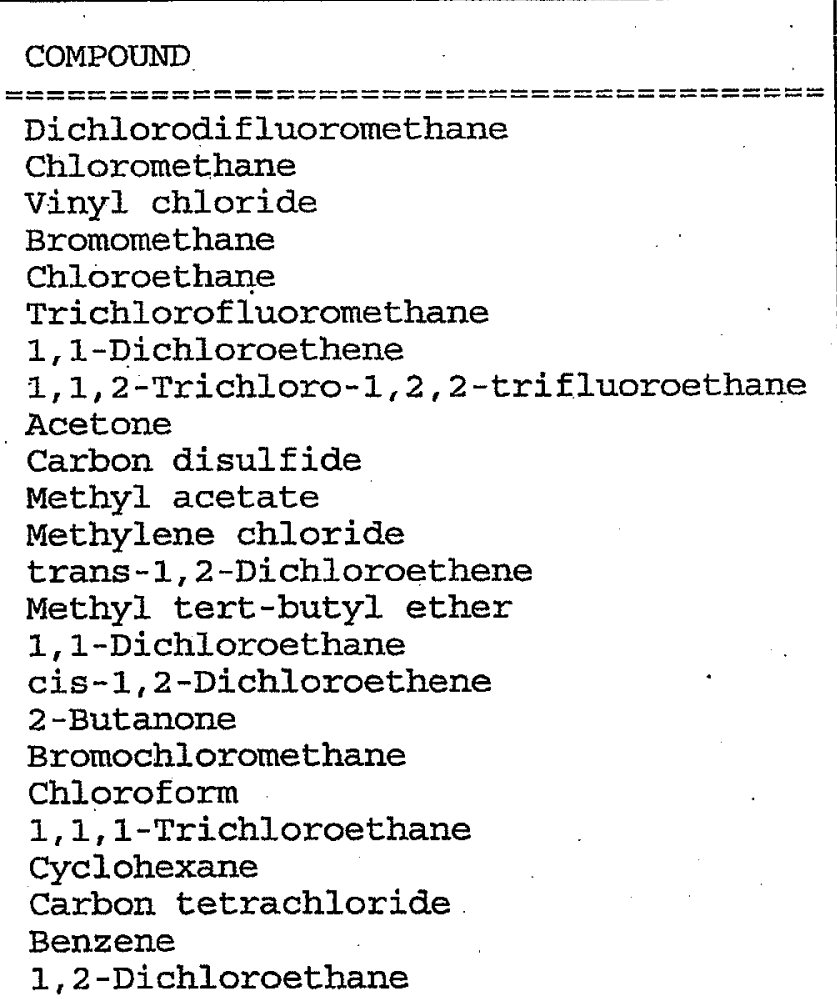 & 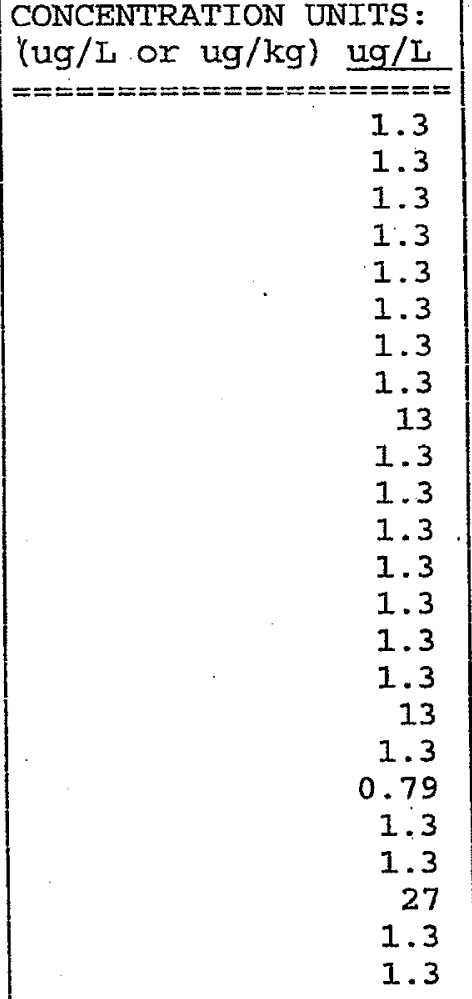 & $\begin{array}{l}========1 \\
U \\
U \\
U \\
U \\
U \\
U \\
U \\
U \\
U \\
U \\
U \\
U \\
U \\
U \\
U \\
U \\
U \\
U \\
D J \\
U \\
U \\
D \\
U \\
U\end{array}$ \\
\hline
\end{tabular}

Report 1,4-Dioxane for Low-Medium VOA analysis only 
$1 B$ - FORM I VOA-2

VOLATILE ORGANICS ANALYSIS DATA SHEET

EPA SAMPLE NO

11SW2765IDL

Lab Name: TESTAMERICA BURLINGTON

Contract: $8 \mathrm{E}-00302$

Lab Code: STLV Case No.: MORRILI Mod. Ref No.:

SDG NO.: 128326

Matrix: (SOIL/SED/WATER) Water

Lab Sample ID: 772518D1

Sample wt/vol: $25.0 \quad(\mathrm{~g} / \mathrm{mL}) \mathrm{mL}$

Lab File ID: $772518 D$

LeVel: (TRACE/LOW/MED) TRACE

Date Received: $10 / 22 / 2008$

$\div$ Moisture: not dec.

Date Analyzed: 10/24/2008

GC Column: DB-624

ID: 0.53

(mm)

Dilution Factor: 2.5

Soil Extract Volume:

(uL) Soil Aliquot Volume:

(uI)

Purge Volume: 25.0

(m工)

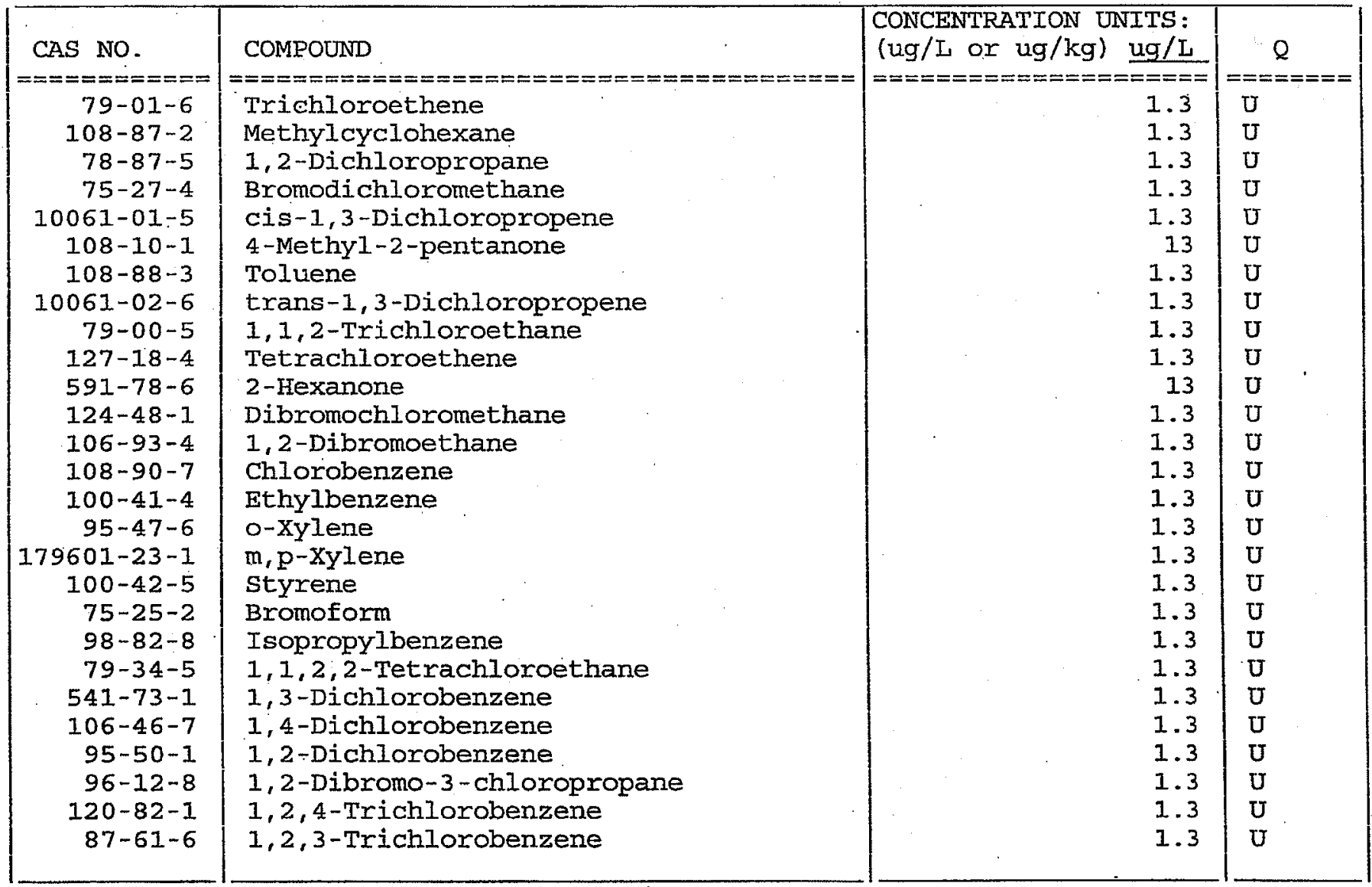


IA - FORM I VOA-I

VOLATILE ORGANICS ANALYSIS DATA SHEET
EPA SAMPLE NO.

ISW 27620
Lab Name: TESTAMERICA BURLINGTON

Lab Code: STIV Case No.: MORRILL

Matrix: (SOIL/SED/WATER) Water

Sample wt/vol: $25.0 \quad(\mathrm{~g} / \mathrm{mL}) \mathrm{mL}$

Level: (TRACE/LOW/MED) TRACE

$\%$ Moisture: not dec.

GC Column: DB-624

ID $: 0.53$

Soil Extract Volume:

Purge Volume: 25.0
Contract: $8 \mathrm{E}-00302$

Mod. Ref No.:
Lab Sample ID: 772511 .

Lab File ID: 7725211

Date Received: 10/22/2008

Date Analyzed: 10/23/2008

(mm) Dilution Factor: 1.0

(uL) Soil Aliquot Volume:

(uL)

(mL)

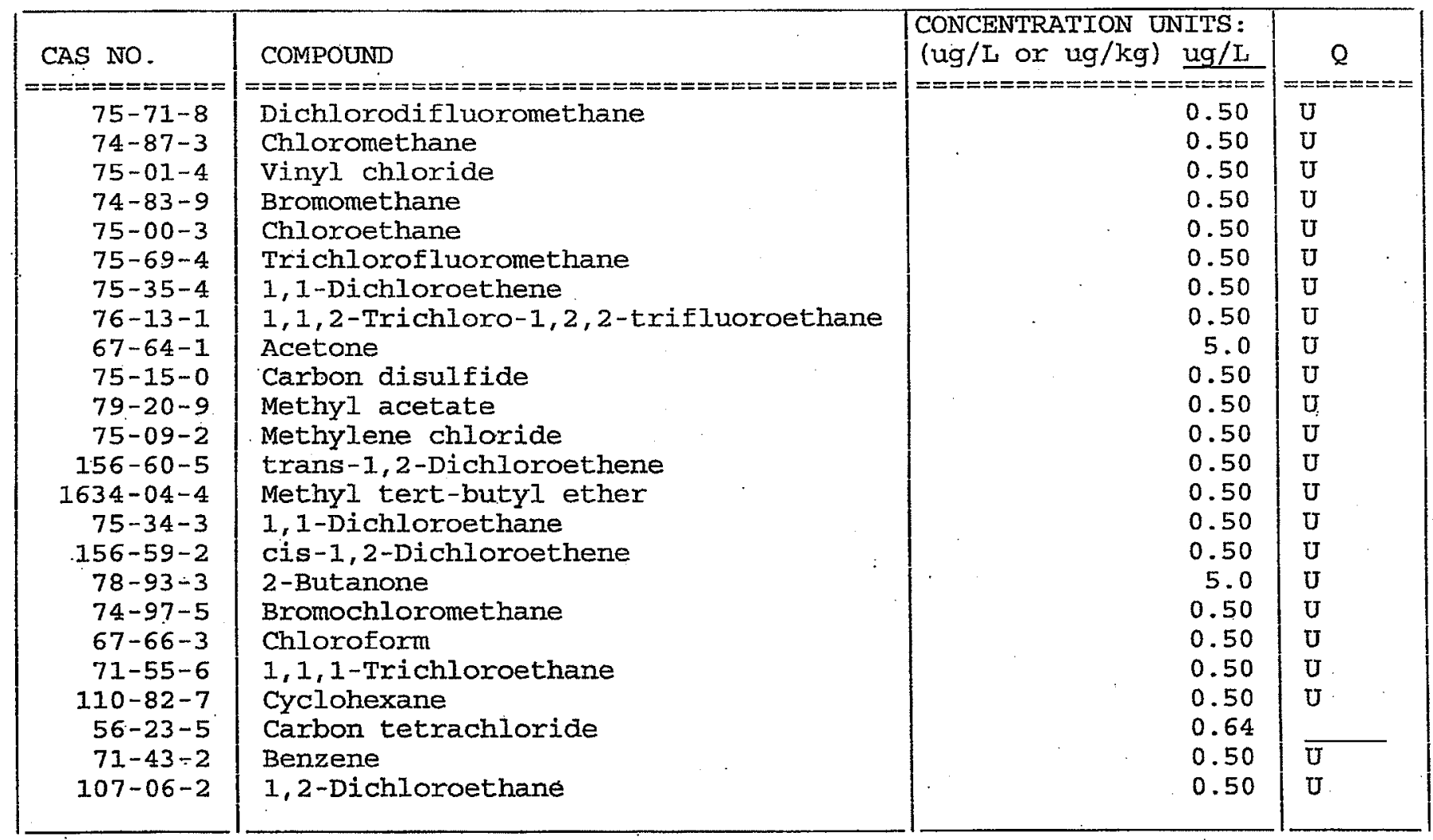

Report 1,4-Dioxane for Low-Medium VOA analysis only 
Lab Name: TESTAMERICA BURLINGTON

Lab Code: STLV Case No.: MORRILI

Matrix: (SOIL/SED/WATER) Water

Sample wt/vol: 25.0

$(\mathrm{g} / \mathrm{mL}) \mathrm{mL}$

Leve1: (TRACE/LOW/MED) TRACE

$\div$ Moisture: not dec.

GC Column: DB-624

Soil Extract Volume:

ID: 0.53

(mm)

(uL)

Contract: $8 E-00302$

Mod. Ref No.:
Lab Sample ID: 772511

Lab File ID: 7725211

Date Received: 10/22/2008

Date Analyzed: 10/23/2008

Dilution Factor: 1.0

Soil Aliquot Volume:

(uL)

Purge Volume: 25.0

(mL)

\begin{tabular}{|c|c|c|}
\hline $\begin{array}{l}\text { CONCENTRATION U } \\
(\mathrm{ug} / \mathrm{L} \text { or } \mathrm{ug} / \mathrm{kg}) \\
=============\end{array}$ & 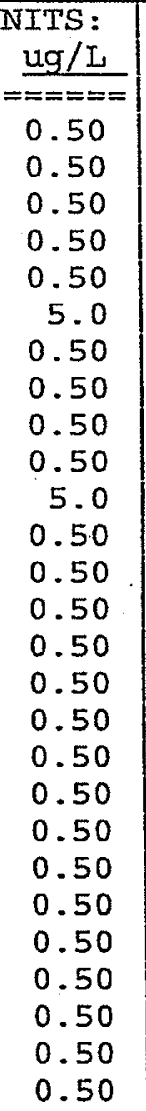 & $\begin{array}{l}======== \\
U \\
U \\
U \\
U \\
U \\
U \\
U \\
U \\
U \\
U \\
U \\
U \\
U \\
U \\
U \\
U \\
U \\
U \\
U \\
U \\
U \\
U \\
U \\
U \\
U \\
U \\
U\end{array}$ \\
\hline
\end{tabular}


IA - FORM I VOA-1

VOLATILE ORGANICS ANALYSIS DATA SHEET
EPA SAMPLE NO

ISW27649
Lab Name: TESTAMERICA BURLINGTON

Lab Code: STLV Case No.: MORRILL

Matrix: (SOIL/SED/WATER) Water

Sample wt/vol: 25.0

$(\mathrm{g} / \mathrm{mL}) \mathrm{mL}$

Level: (TRACE/LOW/MED) TRACE

\% Moisture: not dec.

GC Column: DB-624

Soil Extract Volume:

ID : 0.53

Contract: $8 \mathrm{E}-00302$

Mod. Ref No.:

Purge Volume: 25.0

$(\mathrm{mm})$

(uL)

Lab Sample ID: 772516

Lab File ID: 772516

Date Received: $10 / 22 / 2008$

Date Analyzed: 10/23/2008

Dilution Factor: 1.0

Soil Aliquot Volume:

(UI)

(m工)

\begin{tabular}{|c|c|c|c|}
\hline $\begin{array}{c}\text { CAS NO. } \\
========== \\
75-71-8 \\
74-87-3 \\
75-01-4 \\
74-83-9 \\
75-00-3 \\
75-69-4 \\
75-35-4 \\
76-13-1 \\
67-64-1 \\
75-15-0 \\
79-20-9 \\
75-09-2 \\
156-60-5 \\
1634-04-4 \\
75-34-3 \\
156-59-2 \\
78-93-3 \\
74-97-5 \\
67-66-3 \\
71-55-6 \\
110-82-7 \\
56-23-5 \\
71-43-2 \\
107-06-2\end{array}$ & $\begin{array}{l}\text { COMPoUND } \\
============================= \\
\text { Dichlorodifluoromethane } \\
\text { Chloromethane } \\
\text { Vinyl chloride } \\
\text { Bromomethane } \\
\text { Chloroethane } \\
\text { Trichlorofluoromethane } \\
1,1-D i c h l o r o e t h e n e \\
1,1,2-\text { Trichloro-1, } 2,2-t r i f l u o r o e t h a n e \\
\text { Acetone } \\
\text { Carbon disulfide } \\
\text { Methyl acetate } \\
\text { Methylene chloride } \\
\text { trans-1,2-Dichloroethene } \\
\text { Methyl tert-butyl ether } \\
1,1-D i c h l o r o e t h a n e \\
\text { cis-1,2-Dichloroethene } \\
2-\text { Butanone } \\
\text { Bromochloromethane } \\
\text { Chloroform } \\
1,1,1-\text { Trichloroethane } \\
\text { Cyclohexane } \\
\text { Carbon tetrachloride } \\
\text { Benzene } \\
1,2-D i c h l o r o e t h a n e\end{array}$ & \begin{tabular}{|cc} 
CONCENTRATION UNITS: \\
(ug $/ \mathrm{L}$ or ug $/ \mathrm{kg})$ & ug $/ \mathrm{L}$ \\
$=================$ \\
0.50 \\
0.50 \\
0.50 \\
0.50 \\
0.50 \\
0.50 \\
0.50 \\
0.50 \\
5.0 \\
0.50 \\
0.50 \\
0.50 \\
0.50 \\
0.50 \\
0.50 \\
0.50 \\
.5 .0 \\
0.50 \\
1.6 \\
0.50 \\
0.50 \\
28 \\
0.50 \\
0.50
\end{tabular} & \begin{tabular}{|l}
$=======$ \\
$U$ \\
$U$ \\
$U$ \\
$U$ \\
$U$ \\
$U$ \\
$U$ \\
$U$ \\
$U$ \\
$U$ \\
$U$ \\
$U$ \\
$U$ \\
$U$ \\
$U$ \\
$U$ \\
$U$ \\
$U$ \\
$U$ \\
$U$ \\
$E$ \\
$U$ \\
$U$
\end{tabular} \\
\hline
\end{tabular}

Report 1,4-Dioxane for Low-Medium VOA analysis only 
IB - FORM I VOA-2

VOLATILE ORGANICS ANALYSIS DATA SHEET

EPA SAMPLE NO.

Lab Name: TESTAMERICA BURLINGTON

Contract: 8E-00302

Lab Code: STLV Case No.: MORRILL Mod. Ref No.:

SDG NO.: 128326

Matrix: (SOIL/SED/WATER) Water

Sample wt/vol: $25.0 \quad(\mathrm{~g} / \mathrm{mL}) \mathrm{mL}$

Level: (TRACE/LOW/MED) TRACE

$\%$ Moisture: not dec.

GC Column: DB-624

ID: 0.53

(mm)

(uI)

Lab Sample ID: 772516

Soil Extract Volume:

(mL)

Purge Volume: 25.0
Lab File ID: 772516

Date Received: 10/22/2008

Date Analyzed: 10/23/2008

Dilution Factor: 1.0

Soil Aliquot Volume:

(uI)

\begin{tabular}{|c|c|c|c|}
\hline $\begin{array}{l}\text { CAS NO. } \\
========== \\
79-01-6 \\
108-87-2 \\
78-87-5 \\
75-27-4 \\
10061-01-5 \\
108-10-1 \\
108-88-3 \\
10061-02-6 \\
79-00-5 \\
127-18-4 \\
591-78-6 \\
124-48-1 \\
106-93-4 \\
108-90-7 \\
100-41-4 \\
95-47-6 \\
179601-23-1 \\
100-42-5 \\
75-25-2 \\
98-82-8 \\
79-34-5 \\
541-73-1 \\
106-46-7 \\
95-50-1 \\
96-12-8 \\
120-82-1 \\
87-61-6\end{array}$ & $\begin{array}{l}\text { COMPOUND } \\
\text { Trichloroethene } \\
\text { Methylcyclohexane } \\
\text { 1,2-Dichloropropane } \\
\text { Bromodichloromethane } \\
\text { cis-1,3-Dichloropropene } \\
\text { 4-Methyl-2-pentanone } \\
\text { Toluene } \\
\text { trans-1,3-Dichloropropene } \\
1,1,2-\text { Trichloroethane } \\
\text { Tetrachloroethene } \\
2-\text { Hexanone } \\
\text { Dibromochloromethane } \\
1,2-\text { Dibromoethane } \\
\text { Chlorobenzene } \\
\text { Ethylbenzene } \\
\text { o-Xylene } \\
\text { m, p-Xylene } \\
\text { Styrene } \\
\text { Bromoform } \\
\text { Isopropylbenzene } \\
1,1,2,2-\text { Tetrachloroethane } \\
1,3-\text { Dichlorobenzene } \\
1,4-\text { Dichlorobenzene } \\
1,2-\text { Dichlorobenzene } \\
1,2-D i b r o m o-3-c h l o r o p r o p a n e \\
1,2,4-T r i c h l o r o b e n z e n e \\
1,2,3-\text { Trichlorobenzene }\end{array}$ & 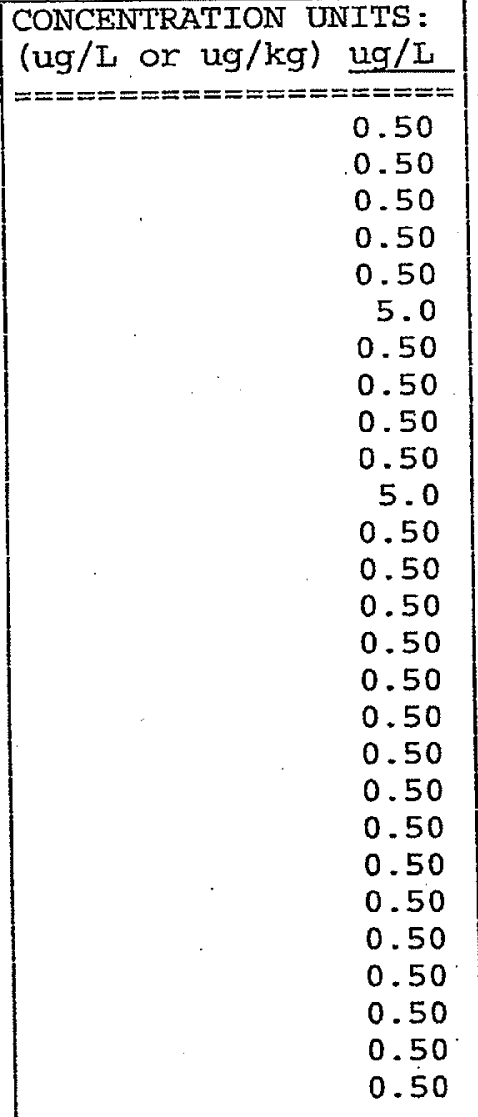 & \begin{tabular}{|l|}
$=======$ \\
$U$ \\
$U$ \\
$U$ \\
$U$ \\
$U$ \\
$U$ \\
$U$ \\
$U$ \\
$U$ \\
$U$ \\
$U$ \\
$U$ \\
$U$ \\
$U$ \\
$U$ \\
$U$ \\
$U$ \\
$U$ \\
$U$ \\
$U$ \\
$U$ \\
$U$ \\
$U$ \\
$U$ \\
$U$ \\
$U$ \\
$U$
\end{tabular} \\
\hline
\end{tabular}


$I A$ - FORM I VOA-1

VOLATILE ORGANICS ANAIYSIS DATA SHEET
EPA SAMPLE NO.

ISW27649DL
Lab Name: TESTAMERICA BURLINGTON

Iab Code: STLV Case No.: MORRILI Matrix: (SOIL/SED/WATER) Water

Sample wt/vol: $25.0 \quad(\mathrm{~g} / \mathrm{mL}) \mathrm{mL}$

Leve1: (TRACE/LOW/MED) TRACE

$\%$ Moisture: not dec.

GC Column: DB-624

$\mathrm{ID}: 0.53$

Soil Extract Volume:

Purge Volume: 25.0
Contract: $8 \mathrm{E}-00302$

SDG NO.: 128326

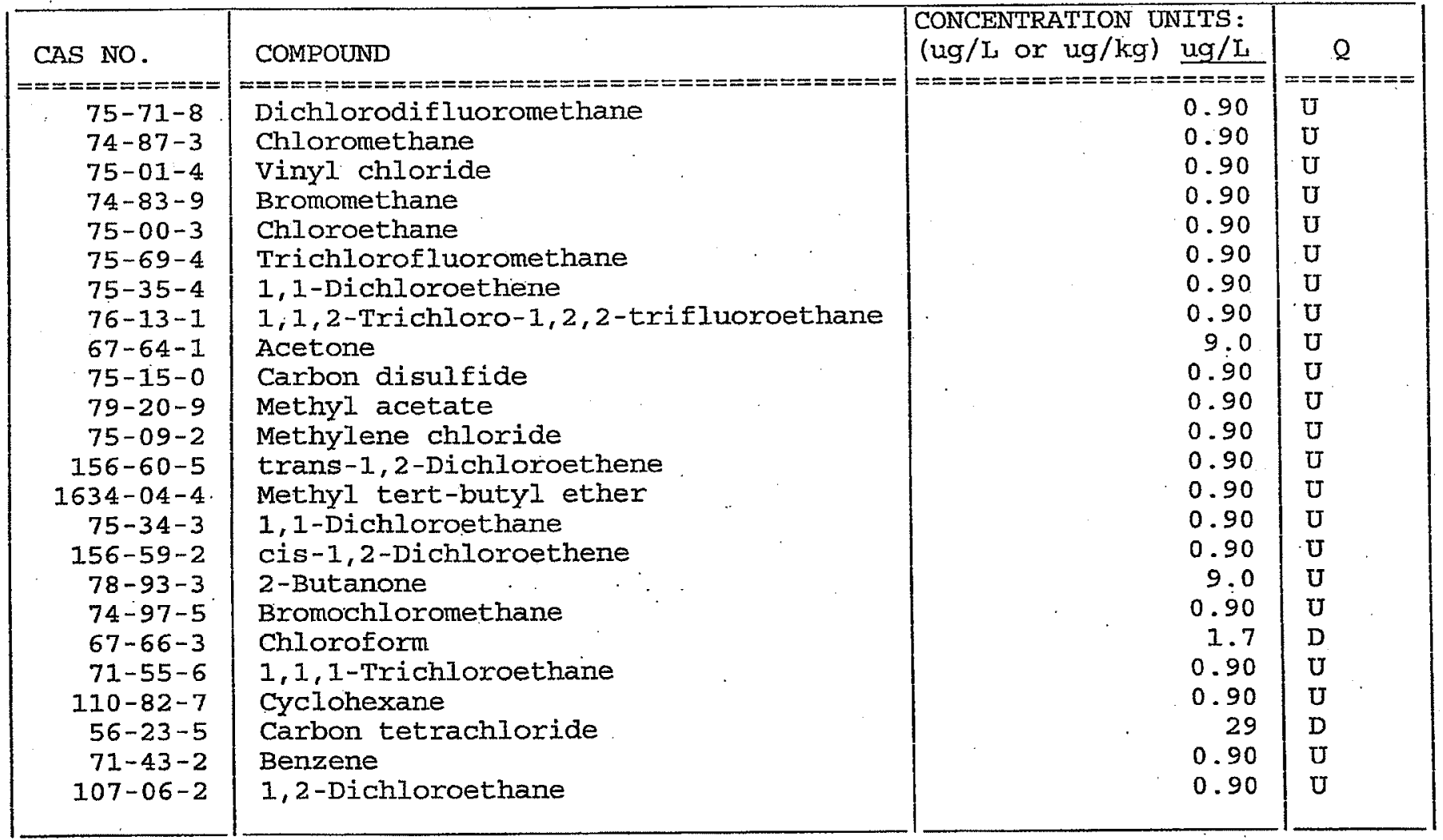

Report 1,4-Dioxane for Low-Medium VOA analysis only 
$I B$ - FORM I VOA-2

VOLATILE ORGANICS ANAIYSIS DATA SHEET

EPA SAMPLE NO.

Lab Name: TESTAMERICA BURLINGTON

Contract: $8 E-00302$

Lab Code: STLV Case No.: MORRILL Mod. Ref No.:

SDG No.: 128326

Matrix: (SOIL/SED/WATER) Water

Lab Sample ID: $772516 D 1$

Sample wt/vol: $25.0 \quad(\mathrm{~g} / \mathrm{mL}) \mathrm{mL}$

Lab File ID: $772516 \mathrm{D}$

Ieve1: (TRACE/LOW/MED) TRACE

Date Received: 10/22/2008

$\%$ Moisture: not dec.

Date Analyzed: 10/24/2008

GC Column: DB-624

ID: $0.53 \quad(\mathrm{~mm})$

Dilution Factor: 1.8

Soil Extract Volume:

(uI) Soil Aliquot Volume:

(uI)

Purge Volume: 25.0

(mI)

\begin{tabular}{|c|c|c|c|}
\hline $\begin{array}{r}\text { CAS NO. } \\
========== \\
79-01-6 \\
108-87-2 \\
78-87-5 \\
75-27-4 \\
10061-01-5 \\
108-10-1 \\
108-88-3 \\
10061-02-6 \\
79-00-5 \\
127-18-4 \\
591-78-6 \\
124-48-1 \\
106-93-4 \\
108-90-7 \\
100-41-4 \\
95-47-6 \\
179601-23-1 \\
100-42-5 \\
75-25-2 \\
98-82-8 \\
79-34-5 \\
541-73-1 \\
106-46-7 \\
95-50-1 \\
96-12-8 \\
120-82-1 \\
87-61-6\end{array}$ & 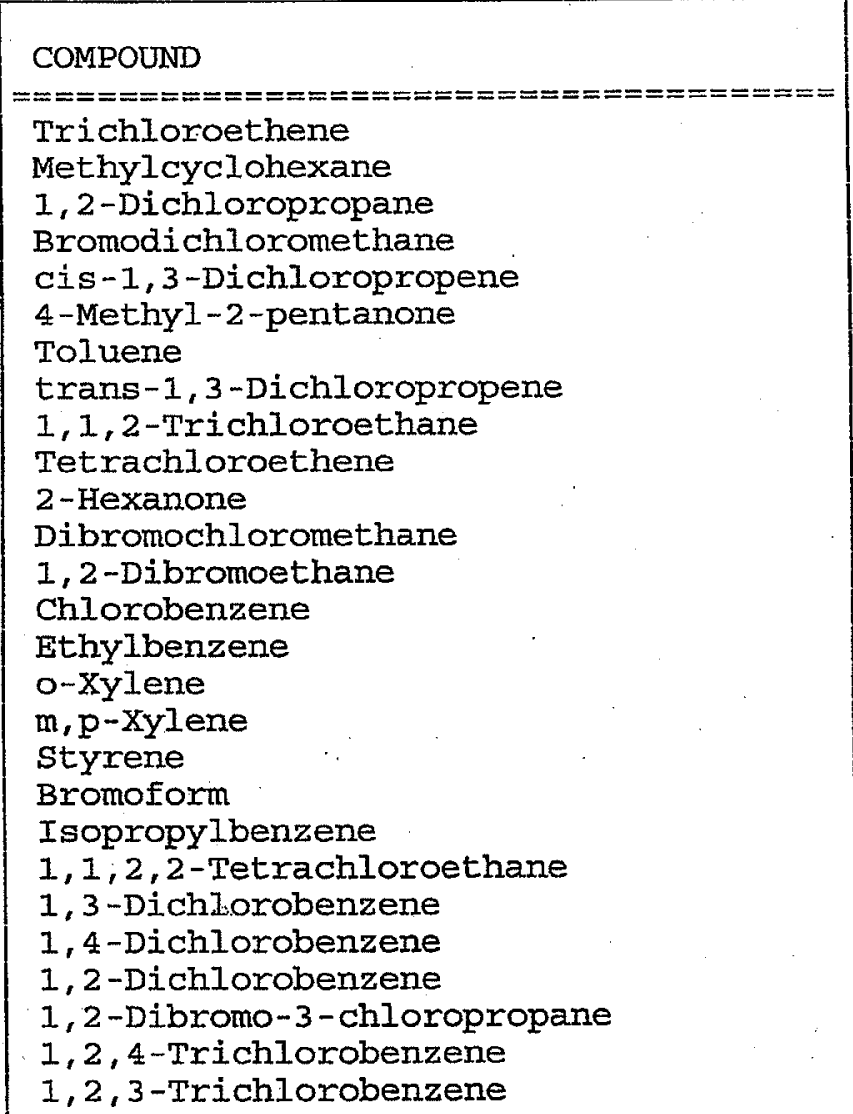 & \begin{tabular}{|cc} 
CONCENTRATION UNTTS: \\
(ug/L OI ug/kg) \\
$====================$ \\
0.90 \\
0.90 \\
0.90 \\
0.90 \\
0.90 \\
9.0 \\
0.90 \\
0.90 \\
0.90 \\
0.90 \\
9.0 \\
0.90 \\
0.90 \\
0.90 \\
0.90 \\
0.90 \\
0.90 \\
0.90 \\
0.90 \\
0.90 \\
0.90 \\
0.90 \\
0.90 \\
0.90 \\
0.90 \\
0.90 \\
0.90
\end{tabular} & \begin{tabular}{|l} 
Q \\
$U$ \\
$U$ \\
$U$ \\
$U$ \\
$U$ \\
$U$ \\
$U$ \\
$U$ \\
$U$ \\
$U$ \\
$U$ \\
$U$ \\
$U$ \\
$U$ \\
$U$ \\
$U$ \\
$U$ \\
$U$ \\
$U$ \\
$U$ \\
$U$ \\
$U$ \\
$U$ \\
$U$ \\
$U$ \\
$U$ \\
$U$
\end{tabular} \\
\hline
\end{tabular}


$1 A$ - FORM I VOA-I.

VOLATILE ORGANICS ANALYSIS DATA SHEET
EPA SAMPLE NO.
Lab Name: TESTAMERICA BURLINGTON

Lab Code: STLV Case No.: MORRILI

Matrix: (SOIL/SED/WATER). Water

Sample wt/vol: $25.0 \quad(\mathrm{~g} / \mathrm{mL}) \mathrm{mL}$

Level: (TRACE/LOW/MED) TRACE

$\div$ Moisture: not dec.

GC Column: DB-624

ID : 0.53

$(\mathrm{mm})$

(uL)

Contract: $8 \mathrm{E}-00302$

Mod. Ref No.:

SDG NO.: 128326
Lab Sample ID: 772514

Lab File ID: 772514

Date Received: 10/22/2008

Date Analyzed: 10/24/2008

Dilution Factor: 1.0

Soil Aliquot Volume:

(uL)
Soil Extract Volume:

Purge Volume: 25.0

(mL)

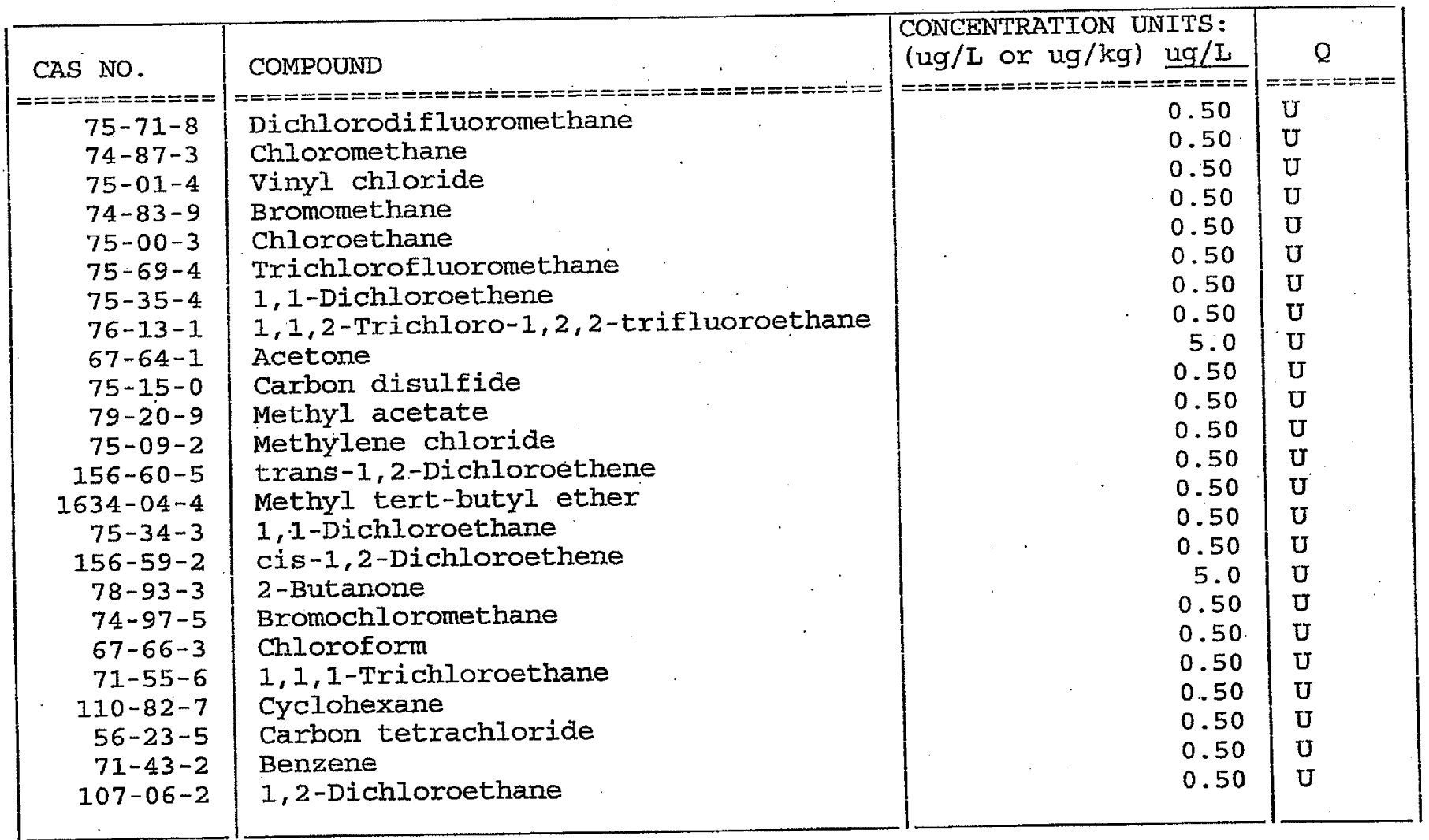

Report 1,4-Dioxane for Low-Medium VOA analysis only 
$1 B$ - FORM I VOA-2

VOIATILE ORGANICS ANAIYSIS DATA SHEET
EPA SAMPLE NO.

2SW2 7622
Iab Name: TESTAMERICA BURIINGTON

Lab Code: STLV Case No.: MORRIIL

Matrix: (SOIL/SED/WATER) Water

Sample wt/vol: $25.0 \quad(\mathrm{~g} / \mathrm{mL}) \mathrm{mL}$

Leve1: (TRACE/LOW/MED) TRACE

$\%$ Moisture: not dec.

GC Column: $\mathrm{DB}-624$

Soil Extract Volume:

ID $: 0.53$

(mm)

(uL)

Contract: $8 E-00302$

Mod. Ref No. :

Lab Sample ID: 772514

Lab File ID: 772514

Dilution Factor: 1.0

Soil Aliquot Volume:
SDG NO.: 128326

Purge Volume: 25.0

Date Received: 10/22/2008

Date Analyzed: 10/24/2008

(uL)

(mL)

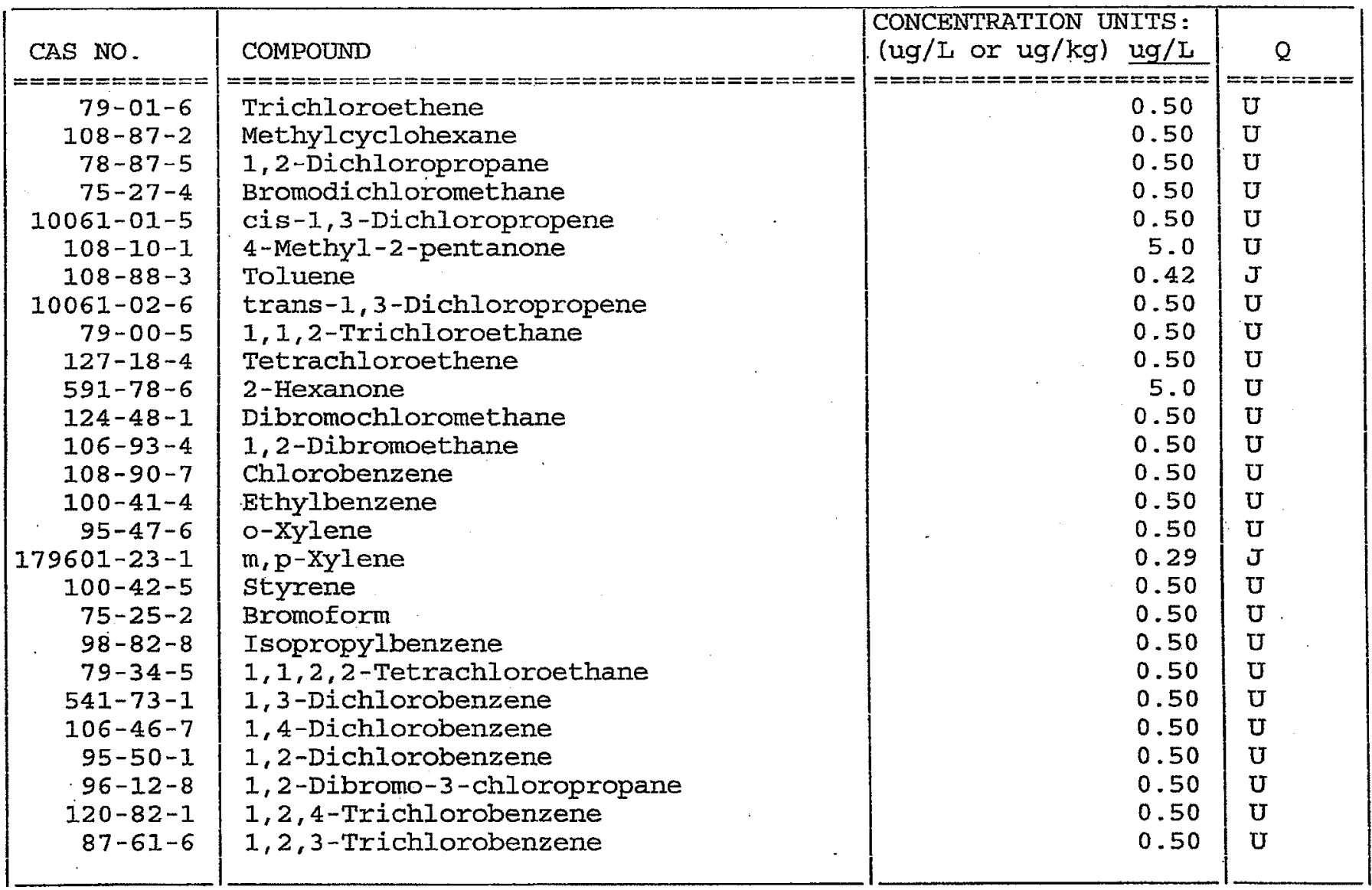

SOM01. 2 
$1 A$ - FORM I VOA-I

VOLATILE ORGANICS ANAIYSIS DATA SHEET
EPA SAMPLE NO.
Lab Name: TESTAMERICA BURLINGTON Lab Code: STLV Case No.: MORRILL Matrix: (SOIL/SED/WATER) Water

Sample wt/vol: 25.0

$(\mathrm{g} / \mathrm{mL}) \mathrm{mL}$

Level: (TRACE/LOW/MED) TRACE

\% Moisture: not dec.

GC Column: DB-624

ID : 0.53

(mm)

(uL)

Contract: 8E-00302

Mod. Ref No.:
Soil Extract Volume:

Purge Volume: 25.0
Lab Sample ID: 772519

Lab File ID: 772519

Date Received: 10/22/2008

Date Analyzed: 10/24/2008

Dilution Factor: 1.0

Soil Aliquot Volume:

(UI)

\begin{tabular}{|c|c|c|c|}
\hline $\begin{array}{c}\text { CAS NO. } \\
=========== \\
75-71-8 \\
74-87-3 \\
75-01-4 \\
74-83-9 \\
75-00-3 \\
75-69-4 \\
75-35-4 \\
76-13-1 \\
67-64-1 \\
75-15-0 \\
79-20-9 \\
75-09-2 \\
156-60-5 \\
1634-04-4 \\
75-34-3 \\
156-59-2 \\
78-93-3 \\
74-97-5 \\
67-66-3 \\
71-55-6 \\
110-82-7 \\
56-23-5 \\
71-43-2 \\
107-06-2\end{array}$ & $\begin{array}{l}\text { COMPOUND } \\
\text { Dichlorodifluoromethane } \\
\text { Chloromethane } \\
\text { Vinyl chloride } \\
\text { Bromomethane } \\
\text { Chloroethane } \\
\text { Trichlorofluoromethane } \\
1,1-D i \text { chloroethene } \\
1,1,2-\text { Trichloro-1,2,2-trifluoroethane } \\
\text { Acetone } \\
\text { Carbon disulfide } \\
\text { Methyl acetate } \\
\text { Methylene chloride } \\
\text { trans-1,2-Dichloroethene } \\
\text { Methyl tert-butyl ether } \\
1,1-D i c h l o r o e t h a n e \\
\text { cis-1, } 2-D i c h l o r o e t h e n e \\
2-\text { Butanone } \\
\text { Bromochloromethane } \\
\text { Chloroform } \\
1,1,1-\text { Trichloroethane } \\
\text { Cyclohexane } \\
\text { Carbon tetrachloride } \\
\text { Benzene } \\
1,2-D i c h l o r o e t h a n e\end{array}$ & 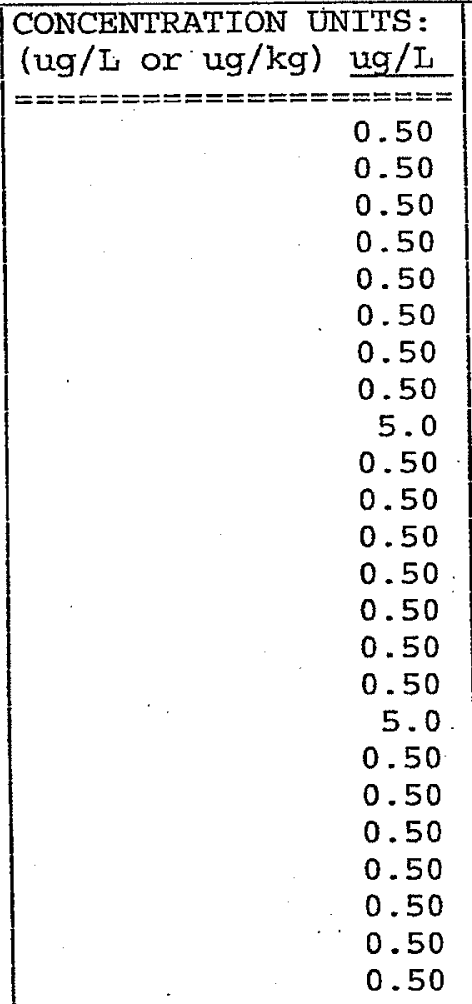 & $\begin{array}{l}======= \\
U \\
U \\
U \\
U \\
U \\
U \\
U \\
U \\
U \\
U \\
U \\
U \\
U \\
U \\
U \\
U \\
U \\
U \\
U \\
U \\
U \\
U \\
U \\
U\end{array}$ \\
\hline
\end{tabular}

Report 1,4-Dioxane for Low-Medium VOA analysis only 
$1 B$ - FORM I VOA-2

VOLATILE ORGANICS ANALYSIS DATA SHEET

EPA SAMPLE NO.

Lab Name: TESTAMERICA BURLINGTON

Contract: $8 \mathrm{E}-00302$

Lab Code: STLV Case No.: MORRILL Mod. ReE No.:

SDG NO. : 128326

Matrix: (SOIL/SED/WATER) Water

Sample wt/vol: $25.0 \quad(\mathrm{~g} / \mathrm{mL}) \mathrm{mL}$

LeVel: (TRACE/LOW/MED) TRACE

\% Moisture: not dec.

GC Column: DB-624

Soil Extract Volume:

ID : $0.53 \quad(\mathrm{~mm})$

(uL)

Lab Sample ID: 772519

Lab File ID: 772519

Date Received: $10 / 22 / 2008$

Date Analyzed: 10/24/2008

Purge Volume: 25.0

(mL)
(uL)

\begin{tabular}{|c|c|c|c|}
\hline $\begin{array}{r}\text { CAS NO. } \\
=========== \\
79-01-6 \\
108-87-2 \\
78-87-5 \\
75-27-4 \\
10061-01-5 \\
108-10-1 \\
108-88-3 \\
10061-02-6 \\
79-00-5 \\
127-18-4 \\
591-78-6 \\
124-48-1 \\
106-93-4 \\
108-90-7 \\
100-41-4 \\
95-47-6 \\
179601-23-1 \\
100-42-5 \\
75-25-2 \\
98-82-8 \\
79-34-5 \\
541-73-1 \\
106-46-7 \\
95-50-1 \\
96-12-8 \\
120-82-1 \\
87-61-6\end{array}$ & 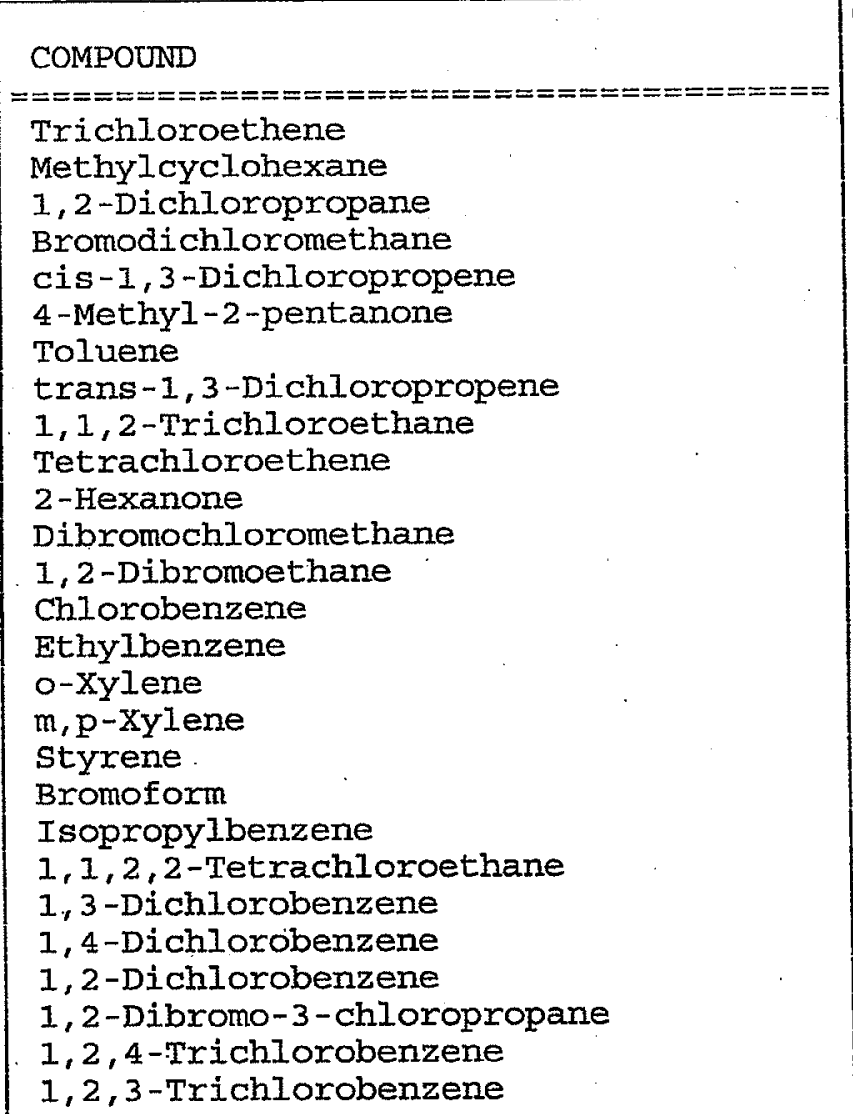 & \begin{tabular}{|cc} 
CONCENTRATION UNITS: \\
(ug $/ \mathrm{L}$ OI ug/kg) & $\underline{u g} / \mathrm{L}$ \\
$===============$ \\
0.50 \\
0.50 \\
0.50 \\
0.50 \\
0.50 \\
5.0 \\
0.50 \\
0.50 \\
0.50 \\
0.50 \\
5.0 \\
0.50 \\
0.50 \\
0.50 \\
0.50 \\
0.50 \\
0.50 \\
0.50 \\
0.50 \\
0.50 \\
0.50 \\
0.50 \\
0.50 \\
0.50 \\
0.50 \\
0.50 \\
0.50
\end{tabular} & $\begin{array}{l}\text { Q } \\
======== \\
U \\
U \\
U \\
U \\
U \\
U \\
U \\
U \\
U \\
U \\
U \\
U \\
U \\
U \\
U \\
U \\
U \\
U \\
U \\
U \\
U \\
U \\
U \\
U \\
U \\
U\end{array}$ \\
\hline
\end{tabular}


$1 A$ - FORM I VOA-1

VOLATIIE ORGANICS ANALYSIS DATA SHEET

EPA SAMPLE NO.

Lab Name: TESTAMERICA BURIINGTON

Contract: $8 E-00302$

Lab Code: STLV Case No.: MORRILI Mod. Ref No.:

SDG No. : 128326

Matrix: (SOII/SED/WATER) Water

Sample wt/vol: $25.0 \quad(\mathrm{~g} / \mathrm{mL}) \mathrm{mL}$

Level: (TRACE/LOW/MED) TRACE

: Moisture: not dec.

GC Column: DB-624

ID $: 0.53 \quad(\mathrm{~mm})$

(UI)

Lab Sample ID: 772515

Lab File ID: 772515

Date Received: 10/22/2008

Date Analyzed: 10/23/2008

Soil Extract Volume:

(mL)

Purge Volume: 25.0

Dilution Factor: 1.0

Soil Aliquot Volume:

(uI)

\begin{tabular}{|c|c|c|c|}
\hline $\begin{array}{c}\text { CAS NO. } \\
=========== \\
75-71-8 \\
74-87-3 \\
75-01-4 \\
74-83-9 \\
75-00-3 \\
75-69-4 \\
75-35-4 \\
76-13-1 \\
67-64-1 \\
75-15-0 \\
79-20-9 \\
75-09-2 \\
156-60-5 \\
1634-04-4 \\
75-34-3 \\
156-59-2 \\
78-93-3 \\
74-97-5 \\
67-66-3 \\
71-55-6 \\
110-82-7 \\
56-23-5 \\
71-43-2 \\
107-06-2\end{array}$ & $\begin{array}{l}\text { COMPOUND } \\
\text { Dichlorodifluoromethane } \\
\text { Chloromethane } \\
\text { Vinyl chloride } \\
\text { Bromomethane } \\
\text { Chloroethane } \\
\text { Trichlorofluoromethane } \\
1,1-D i c h l o r o e t h e n e \\
1,1,2-\text { Trichloro-1, } 2,2-t r i f l u o r o e t h a n e \\
\text { Acetone } \\
\text { Carbon disulfide } \\
\text { Methyl acetate } \\
\text { Methylene chloride } \\
\text { trans-1, } 2 \text {-Dichloroethene } \\
\text { Methyl tert-butyl ether } \\
\text { 1, } 1-D i c h l o r o e t h a n e \\
\text { cis-1,2-Dichloroethene } \\
2-\text { Butanone } \\
\text { Bromochloromethane } \\
\text { Chloroform } \\
1,1,1-T r i c h l o r o e t h a n e \\
\text { Cyclohexane } \\
\text { Carbon tetrachloride } \\
\text { Benzene } \\
1,2-D i c h l o r o e t h a n e\end{array}$ & \begin{tabular}{|c|} 
CONCENTRATION UNITS: \\
(ug/L or ug/kg) \\
$==================$ \\
0.50 \\
0.50 \\
0.50 \\
0.50 \\
0.50 \\
0.50 \\
0.50 \\
0.50 \\
5.0 \\
0.50 \\
0.50 \\
0.50 \\
0.50 \\
0.50 \\
0.50 \\
0.50 \\
5.0 \\
0.50 \\
1.5 \\
0.50 \\
0.50 \\
51 \\
0.50 \\
0.50
\end{tabular} & $\begin{array}{l}Q \\
======== \\
U \\
U \\
U \\
U \\
U \\
U \\
U \\
U \\
U \\
U \\
U \\
U \\
U \\
U \\
U \\
U \\
U \\
U\end{array}$ \\
\hline
\end{tabular}

Report 1,4-Dioxane for Low-Medium VOA analysis only 
IB - FORM I VOA-2

VOLATILE ORGANICS ANALYSIS DATA SHEET

EPA SAMPLE NO.

Lab Name: TESTAMERICA BURLINGTON

Contract: $8 E-00302$

Lab Code: STLV Case No.: MORRILL Mod. Ref No.:

SDG NO.: 128326

Matrix: (SOIL/SED/WATER) Water

Sample wt/vol: $25.0 \quad(\mathrm{~g} / \mathrm{mL}) \mathrm{mL}$

LeVe1: (TRACE/IOW/MED) TRACE

\% Moisture: not dec.

GC Column: DB-624

ID : 0.53

Soil Extract Volume:

Purge Volume: 25.0
Lab Sample ID: 772515

Lab File ID: 772515

Date Received: $10 / 22 / 2008$

Date Analyzed: 10/23/2008

(mm) Dilution Factor: 1.0

(uL) Soil Aliquot Volume:

(uL)

(mI)

\begin{tabular}{|c|c|c|c|}
\hline $\begin{array}{r}\text { CAS NO. } \\
=========== \\
79-01-6 \\
108-87-2 \\
78-87-5 \\
75-27-4 \\
10061-01-5 \\
108-10-1 \\
108-88-3 \\
10061-02-6 \\
79-00-5 \\
127-18-4 \\
591-78-6 \\
124-48-1 \\
106-93-4 \\
108-90-7 \\
100-41-4 \\
95-47-6 \\
17961-23-1 \\
100-42-5 \\
75-25-2 \\
98-82-8 \\
79-34-5 \\
541-73-1 \\
106-46-7 \\
95-50-1 \\
96-12-8 \\
120-82-1 \\
87-61-6\end{array}$ & 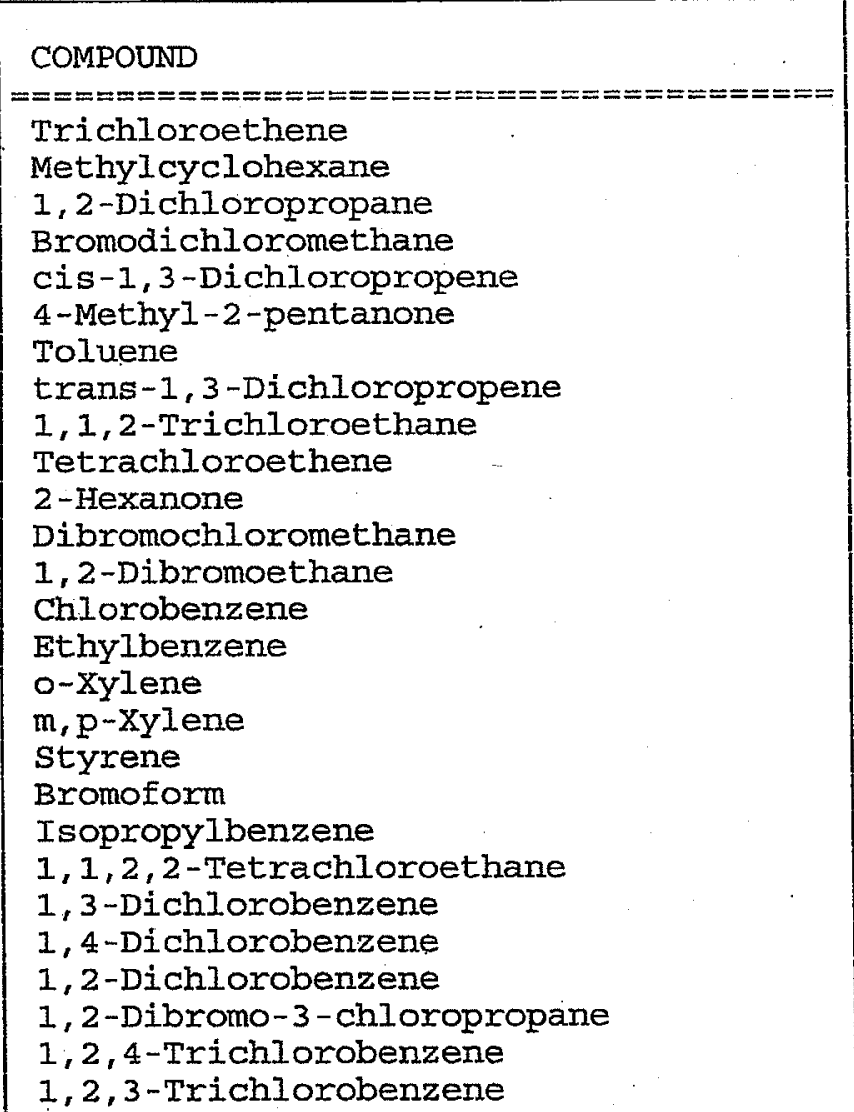 & 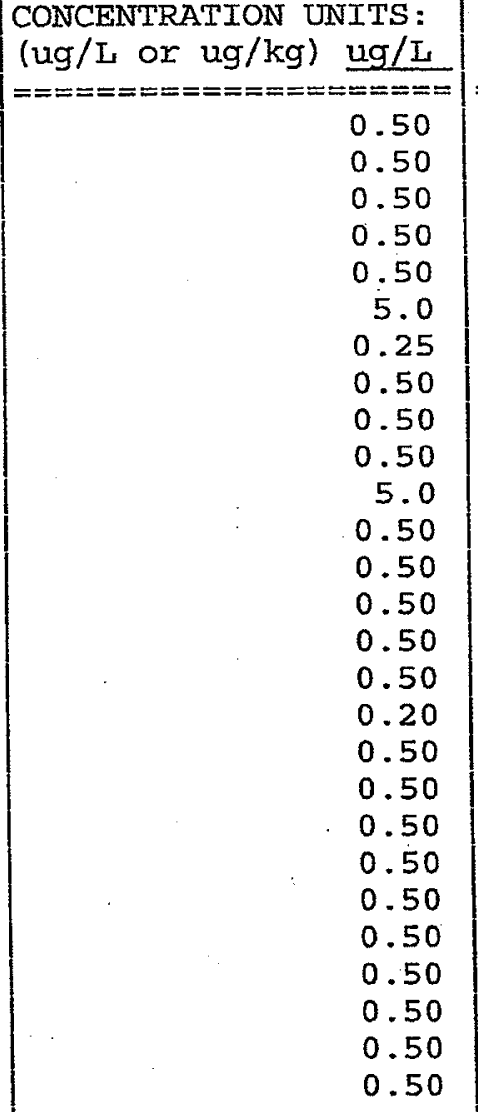 & \begin{tabular}{|l|}
$=======$ \\
$U$ \\
$U$ \\
$U$ \\
$U$ \\
$U$ \\
$U$ \\
$J$ \\
$U$ \\
$U$ \\
$U$ \\
$U$ \\
$U$ \\
$U$ \\
$U$ \\
$U$ \\
$U$ \\
$J$ \\
$U$ \\
$U$ \\
$U$ \\
$U$ \\
$U$ \\
$U$ \\
$U$ \\
$U$ \\
$U$ \\
$U$
\end{tabular} \\
\hline
\end{tabular}


$1 A$ - FORM I VOA-1

VOLATILE ORGANICS ANALYSIS DATA SHEET

EPA SAMPLE NO.

Lab Name: TESTAMERICA BURIINGTON

Contract: $8 E-00302$

Lab Code: STLV Case No.: MORRIII Mod. Ref No.:

SDG No.: 128326

Matrix: (SOIL/SED/WATER) Water

Sample wt/vol: $25.0 \quad(\mathrm{~g} / \mathrm{mL}) \mathrm{mL}$

Level : (TRACE/LOW/MED) TRACE

\% Moisture: not dec.

GC Column: DB-624

ID $: 0.53$

Soil Extract Volume:

Purge Volume: 25.0
Lab Sample ID: 772515D1

Lab File ID: $772515 \mathrm{D}$

Date Receivea: 10/22/2008

Date Analyzed: 10/24/2008

(mm) Dilution Factor: 3.3

(uL) Soil Aliquot Volume:

(uI)

(mL)

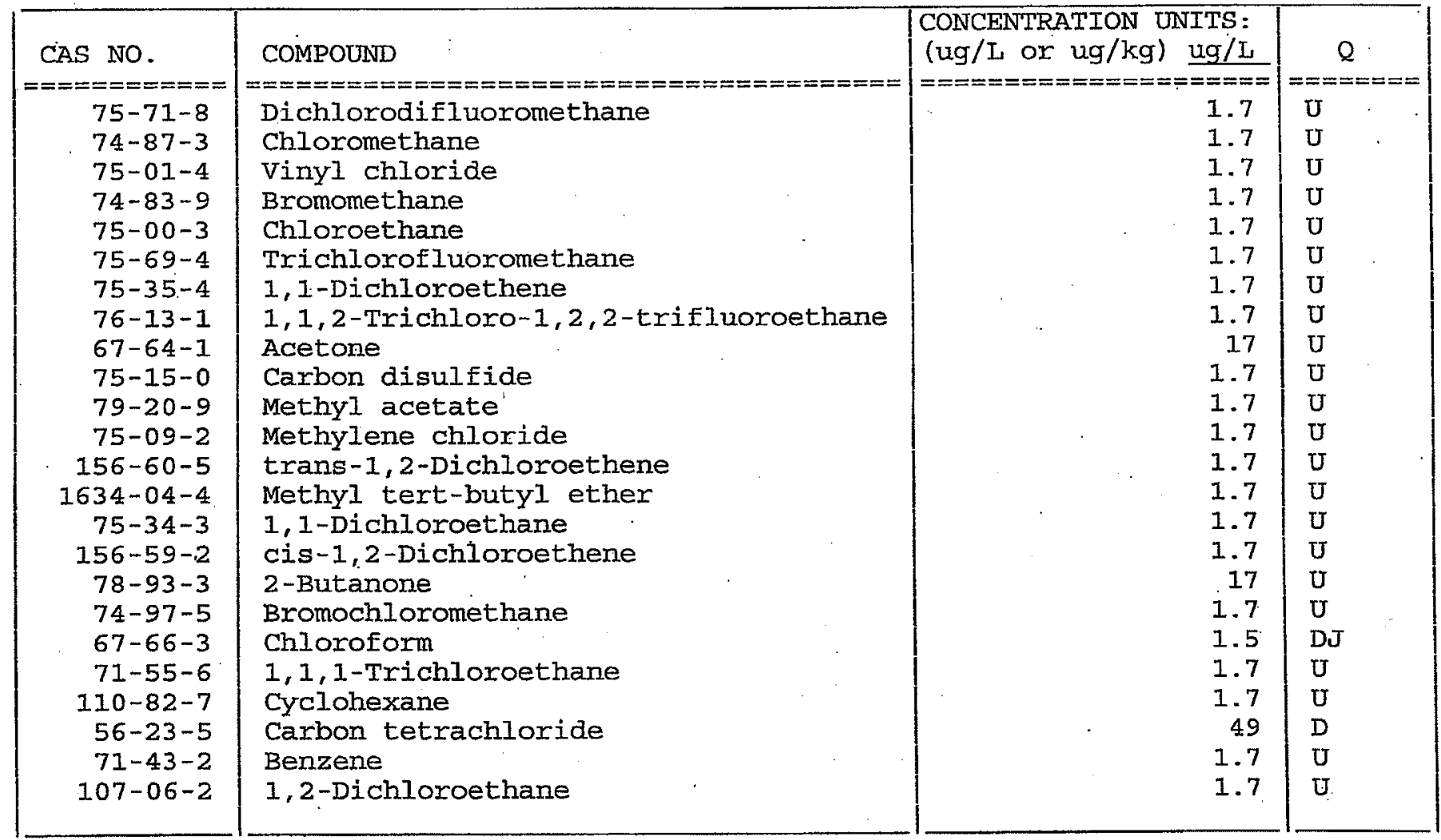

Report 1,4-Dioxane for Low-Medium VOA analysis only 
IB - FORM I VOA-2

EPA SAMPLE NO.

\section{VOLATILE ORGANICS ANALYSIS DATA SHEET}

Lab Name: TESTAMERICA BURLINGTON

Contract: $8 \mathrm{E}-00302$

Lab Code: STLV Case No.: MORRILI Mod. Ref No.:

SDG No.: 128326

Matrix: (SOIL/SED/WATER) Water

Sample wt/vol: $25.0 \quad(\mathrm{~g} / \mathrm{mL}) \mathrm{mL}$

Level: (TRACE/IOW/MED) TRACE

\% Moisture: not dec.

GC Column: DB-624

Soil Extract Volume:

ID $: 0.53$

Purge Volume: 25.0
Lab Sample ID: 772515DI

Lab File ID: $772515 \mathrm{D}$

Date Received: $10 / 22 / 2008$

Date Analyzed: 10/24/2008

(mm) Dilution Factor: 3.3

(uI) soil Aliquot Volume:

(uL)

$(\mathrm{mL})$

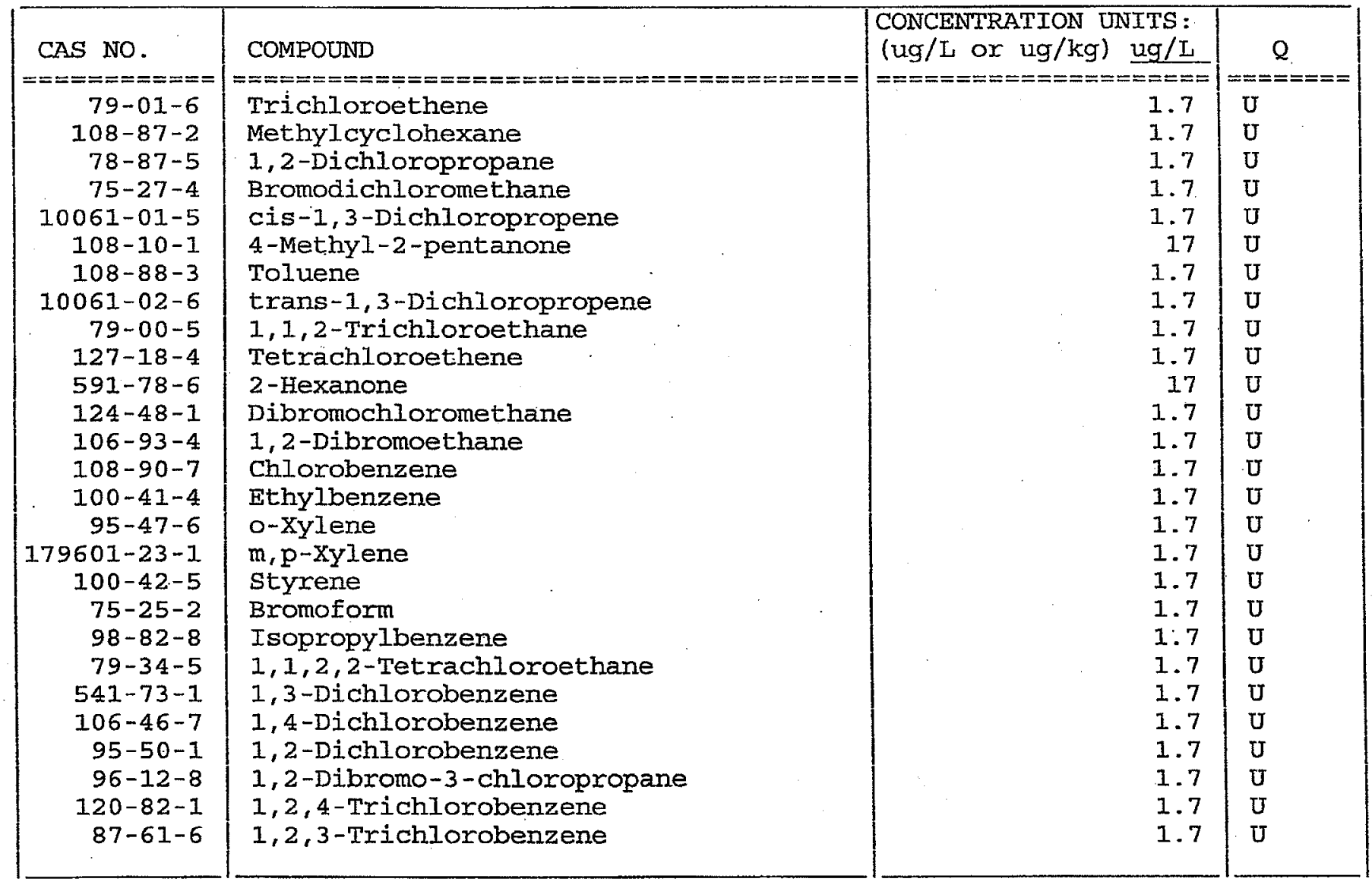


IA - FORM I VOA-I

VOLATILE ORGANICS ANALYSIS DATA SHEET

EPA SAMPLE NO.

Lab Name: TESTAMERICA BURLINGTON

Contract: $8 \mathrm{E}-00302$

Lab Code: STLV Case No.: MORRILL Mod. Ref No.:

SDG NO.: 128326

Matrix: (SOIL/SED/WATER) Water

Lab Sample ID: 772517

Sample wt/vol: $25.0 \quad(\mathrm{~g} / \mathrm{mL}) \mathrm{mL}$

Lab File ID: 772517

Leve1: (TRACE/LOW/MED) TRACE

Date Received: $10 / 22 / 2008$

$\div$ Moisture: not dec.

Date Analyzed: 10/24/2008

GC Column: DB-624

ID : 0.53

(mm)

Dilution Factor: 1.0

Soil Extract Volume:

(uIs)

Soil Aliquot Volume:

(uIs)

Purge Volume: 25.0

(mI)

\begin{tabular}{|c|c|c|c|}
\hline $\begin{array}{l}\text { CAS NO. } \\
========= \\
75-71-8 \\
74-87-3 \\
75-01-4 \\
74-83-9 \\
75-00-3 \\
75-69-4 \\
75-35-4 \\
76-13-1 \\
67-64-1 \\
75-15-0 \\
79-20-9 \\
75-09-2 \\
156-60-5 \\
1634-04-4 \\
75-34-3 \\
156-59-2 \\
78-93-3 \\
74-97-5 \\
67-66-3 \\
71-55-6 \\
110-82-7 \\
56-23-5 \\
71-43-2 \\
107-06-2\end{array}$ & $\begin{array}{l}\text { COMPounD } \\
============================== \\
\text { Dichlorodifluoromethane } \\
\text { Chloromethane } \\
\text { Vinyl chloride } \\
\text { Bromomethane } \\
\text { Chloroethane } \\
\text { Trichlorofluoromethane } \\
1,1-D i c h l o r o e t h e n e \\
1,1,2-\text { Trichloro-1,2,2-trifluoroethane } \\
\text { Acetone } \\
\text { Carbon disulfide } \\
\text { Methyl acetate } \\
\text { Methylene chloride } \\
\text { trans-1,2-Dichloroethene } \\
\text { Methyl tert-butyl ether } \\
1,1-D i c h l o r o e t h a n e \\
\text { cis-1,2-Dichloroethene } \\
2-\text { Butanone } \\
\text { Bromochloromethane } \\
\text { Chloroform } \\
1,1,1-T r i c h l o r o e t h a n e \\
\text { Cyclobexane } \\
\text { Carbon tetrachloride } \\
\text { Benzene } \\
1,2-D i c h l o r o e t h a n e\end{array}$ & $\begin{array}{|cr|}\text { CONCENTRATION UNITS: } \\
\text { (ug } / \mathrm{I} \text { or. ug/kg) } & \mathrm{ug} / \mathrm{L} \\
============= \pm=== \\
0.50 \\
0.50 \\
0.50 \\
0.50 \\
0.50 \\
0.50 \\
0.50 \\
0.50 \\
5.0 \\
0.50 \\
0.50 \\
0.50 \\
0.50 \\
0.50 \\
0.50 \\
0.50 \\
5.0 \\
0.50 \\
1.6 \\
0.50 \\
0.50 \\
56 \\
0.50 \\
0.50\end{array}$ & \begin{tabular}{|c}
$\mathrm{Q}$ \\
$=======$ \\
$\mathrm{U}$ \\
$\mathrm{U}$ \\
$\mathrm{U}$ \\
$\mathrm{U}$ \\
$\mathrm{U}$ \\
$\mathrm{U}$ \\
$\mathrm{U}$ \\
$\mathrm{U}$ \\
$\mathrm{U}$ \\
$\mathrm{U}$ \\
$\mathrm{U}$ \\
$\mathrm{U}$ \\
$\mathrm{U}$ \\
$\mathrm{U}$ \\
$\mathrm{U}$ \\
$\mathrm{U}$ \\
$\mathrm{U}$ \\
$\mathrm{U}$
\end{tabular} \\
\hline
\end{tabular}

Report 1,4-Dioxane for Low-Medium VOA analysis only 
$I B$ - FORM I VOA-2

VOLATILE ORGANICS ANALYSIS DATA SHEET

Lab Name: TESTAMERICA BURIINGTON

Contract: 8E-00302

Lab Code: STLV Case No.: MORRILI Mod. Ref No.:

SDG NO.: 128326

Matrix: (SOIL/SED/WATER) Water

Sample wt/vol: $25.0 \quad(\mathrm{~g} / \mathrm{mL}) \mathrm{mL}$

LeVeI : (TRACE/LOW/MED) TRACE

\% Moisture: not dec.

GC Column: DB-624

ID : $0.53 \quad(\mathrm{~mm})$

(uI) Soil Aliquot Volume:

(UL)

Lab Sample ID: 772517

Lab File ID: 772517

Date Received: 10/22/2008

Date Analyzed: 10/24/2008

Dilution Factor: 1.0

Soil Extract Volume:

(mL)

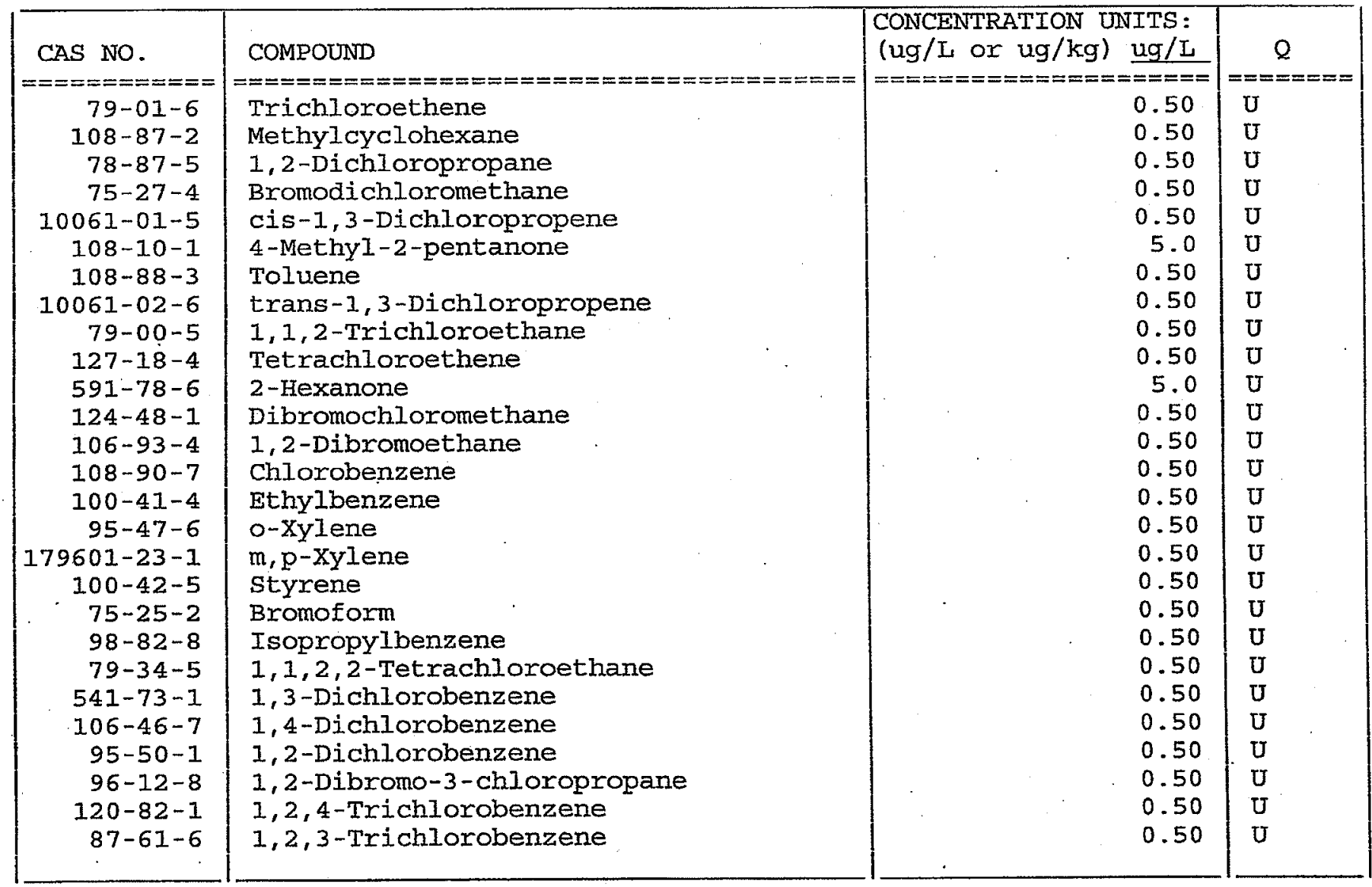


$1 A$ - FORM I VOA-I

VOLATILE ORGANICS ANALYSIS DATA SHEET
EPA SAMPLE NO.
Lab Name: TESTAMERICA BURLINGTONं

Lab Code: STLV Case No.: MORRILL

Matrix: (SOII/SED/WATER) Water

Sample wt/vol: 25.0

$(\mathrm{g} / \mathrm{mI}) \mathrm{mL}$

Level: (TRACE/LOW/MED) TRACE

\% Moisture: not dec.

GC Column: DB-624

ID $: 0.53$

Soil Extract Volume:

Purge Volume: 25.0
Contract $=8 \mathrm{E}-00302$
Lab Sample ID: 772517DI

Lab File ID: $772517 D$

Date Received: 10/22/2008

Date Analyzed: 10/24/2008

Dilution Factor: 3.3

Soil Aliquot Volume:

(UL)

\begin{tabular}{|c|c|c|c|}
\hline $\begin{array}{c}\text { CAS NO. } \\
=========== \\
75-71-8 \\
74-87-3 \\
75-01-4 \\
74-83-9 \\
75-00-3 \\
75-69-4 \\
75-35-4 \\
76-13-1 \\
67-64-1 \\
75-15-0 \\
79-20-9 \\
75-09-2 \\
156-60-5 \\
1634-04-4 \\
75-34-3 \\
156-59-2 \\
78-93-3 \\
74-97-5 \\
67-66-3 \\
71-55-6 \\
110-82-7 \\
56-23-5 \\
71-43-2 \\
107-06-2\end{array}$ & $\begin{array}{l}\text { COMPounD } \\
=============================== \\
\text { Dichlorodifluoromethane } \\
\text { Chloromethane } \\
\text { Vinyl chloride } \\
\text { Bromomethane } \\
\text { Chloroethane } \\
\text { Trichlorofluoromethane } \\
1,1-D i c h l o r o e t h e n e \\
1,1,2-\text { Trichloro-1,2,2-trifluoroethane } \\
\text { Acetone } \\
\text { Carbon disulfide } \\
\text { Methyl acetate } \\
\text { Methylene chloride } \\
\text { trans-1,2-Dichloroethene } \\
\text { Methyl tert-butyl ether } \\
1,1-D i c h l o r o e t h a n e \\
\text { cis-1,2-Dichloroethene } \\
2-\text { Butanone } \\
\text { Bromochloromethane } \\
\text { Chloroform } \\
1,1,1-\text { Trichloroethane } \\
\text { Cyclohexane } \\
\text { Carbon tetrachloride } \\
\text { Benzene } \\
1,2-D i c h l o r o e t h a n e\end{array}$ & 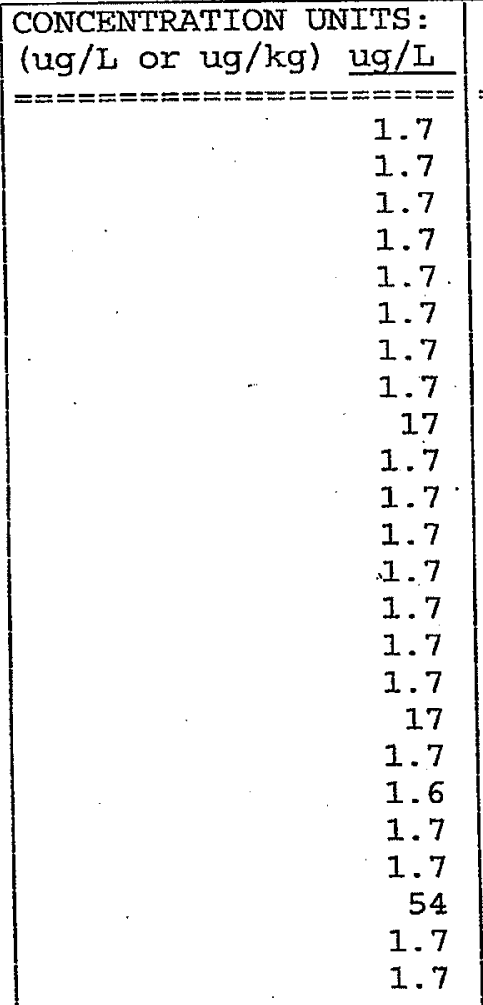 & $\begin{array}{l}\text { Q } \\
======== \\
U \\
U \\
U \\
U \\
U \\
U \\
U \\
U \\
U \\
U \\
U \\
U \\
U \\
U \\
U \\
U \\
U \\
U \\
D J \\
U \\
U \\
D \\
U \\
U\end{array}$ \\
\hline
\end{tabular}

Report 1,4-Dioxane for Low-Medium VOA analysis only 
IB - FORM I VOA-2

VOLATIIE ORGANICS ANAIYYSIS DATA SHEET
EPA SAMPLE NO.

3SW27650DL
Lab Name: TESTAMERICA BURLINGTON

Lab Code: STLV

Case No.: MORRILL

Matrix: (SOIL/SED/WATER) Water

Sample wt/vol: 25.0

$(\mathrm{g} / \mathrm{mL}) \mathrm{mL}$

Level : (TRACE/LOW/MED) TRACE

\% Moisture: not dec.

GC Column: $D B-624$

ID $: 0.53$

(mm)

(uL)

Contract: $8 \mathrm{E}-00302$

Soil Extract Volume:

(mL)
Lab Sample ID: 772517D1

Lab File ID: 772517D

Date Received: 10/22/2008

Date Analyzed: 10/24/2008

Dilution Factor: 3.3

Soil Aliquot Volume:

(uL)

\begin{tabular}{|c|c|c|}
\hline \multicolumn{2}{|c|}{ CONCENTRATION UNITS: } & \multirow{3}{*}{$======$} \\
\hline$(\mathrm{ug} / \mathrm{J}$ or $u g / \mathrm{kg})$ & $\mathrm{ug} / \mathrm{L}$ & \\
\hline 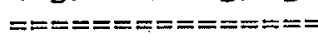 & $== \pm==$ & \\
\hline & 1.7 & $\mathrm{U}$ \\
\hline r. & 1.7 & $\mathrm{U}$ \\
\hline & 1.7 & $U$ \\
\hline & 1.7 & $\mathrm{U}$ \\
\hline & 1.7 & $\mathrm{U}$ \\
\hline & 17 & U \\
\hline & 1.7 & $\mathrm{U}$ \\
\hline & 1.7 & $\mathrm{U}$ \\
\hline & 1.7 & $\mathrm{U}$ \\
\hline & 1.7 & $\mathrm{U}$ \\
\hline & 17 & $\mathrm{U}$ \\
\hline & 1.7 & U \\
\hline & 1.7 & U \\
\hline & 1.7 & U \\
\hline & 1.7 & $\mathrm{U}$ \\
\hline & 1.7 & $\mathrm{U}$ \\
\hline & 1.7 & U \\
\hline . & 1.7 & U \\
\hline & 1.7 & $U$ \\
\hline & 1.7 & U \\
\hline & 1.7 & U \\
\hline & 1.7 & $U$ \\
\hline & 1.7 & U \\
\hline & 1.7 & U \\
\hline & 1.7 & U \\
\hline & 1.7 & $\mathrm{U}$ \\
\hline & 1.7 & U \\
\hline
\end{tabular}


IA - FORM I VOA-I

VOLATILE ORGANICS ANALYSIS DATA SHEET
EPA SAMPLE NO.
Lab Name: TESTAMERICA BURLINGTON

Lab code: STIV Case No.: MORRII

Matrix: (SOIJ/SED/WATER) Water

Sample wt/vol: 25.0

$(\mathrm{g} / \mathrm{mL}) \mathrm{mL}$

Level: (TRACE/LOW/MED) TRACE

\% Moisture: not dec.

GC Column: DB-624

Soil Extract Volume:

ID : 0.53

(mm)

(uL)

Contract: $8 \mathrm{E}-00302$

Mod. Ref No.:

Lab Sample ID: 772512

Lab File ID: 772512
SDG No.: 128326

Purge Volume: 25.0

Date Received: 10/22/2008

Date Analyzed: 10/24/2008

(mI)

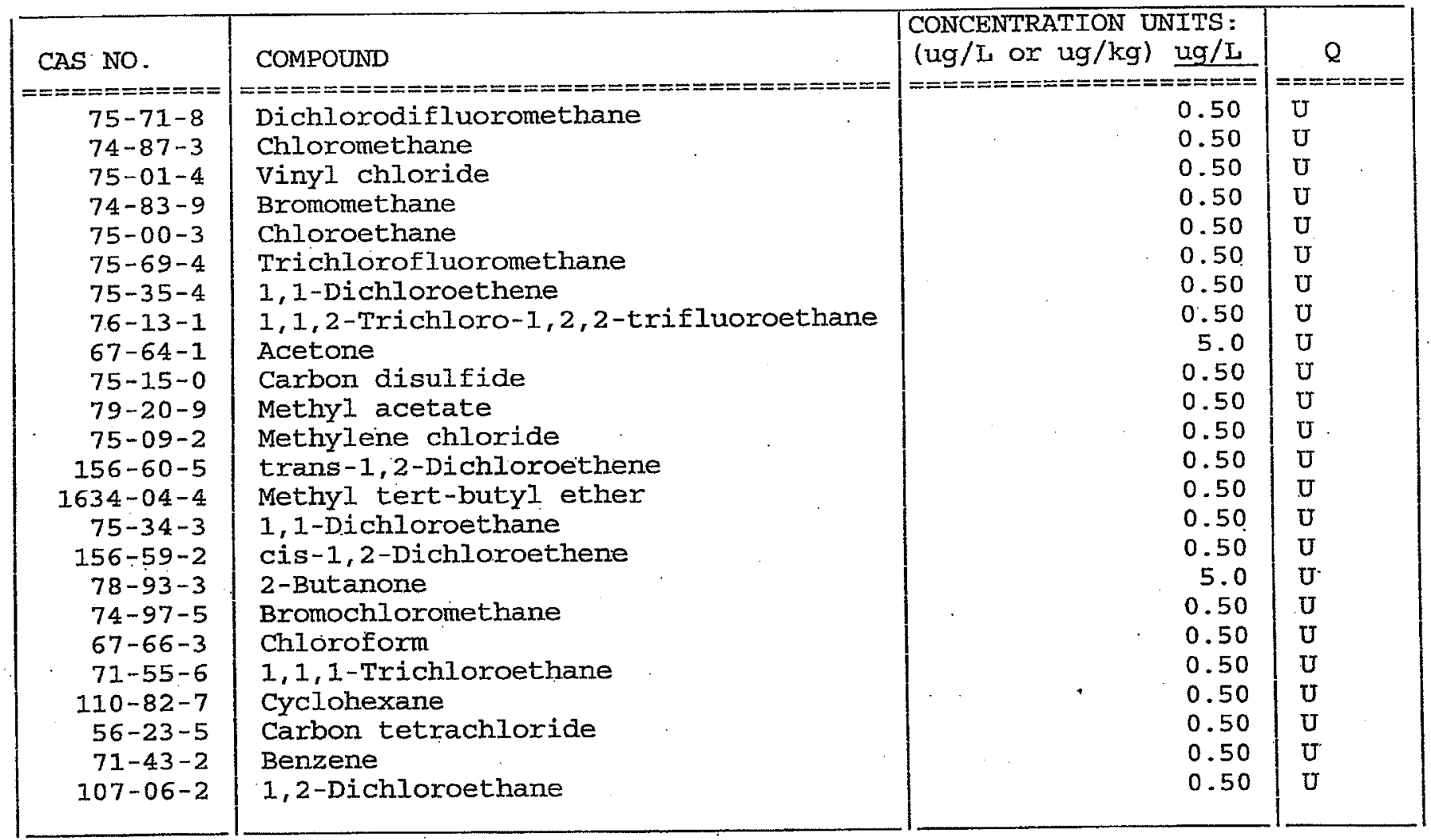

Report 1,4-Dioxane for Low-Medium VOA analysis only 
$1 B$ - FORM I VOA-2

VOTATILE ORGANICS ANALYSIS DATA SHEET

EPÄ SAMPLE NO.

Lab Name: TESTAMERICA BURLINGTON

Contract: $8 \mathrm{E}-00302$

Lab Code: STIV Case No.: MORRILI Mod. Ref No.:

SDG No. : 128326

Matrix: (SOIL/SED/WATER) Water

Sample wt/vol: $25.0 \quad(\mathrm{~g} / \mathrm{mL}) \mathrm{mL}$

Leve 1: (TRACE/LOW/MED) TRACE

: Moisture: not dec.

GC Column: DB-624

ID: $0.53 \quad(\mathrm{~mm})$

(uL)

Lab Sample ID: 772512

Lab File ID: 772512

Date Received: 10/22/2008

Date Analyzed: $10 / 24 / 2008$

Soil Extract Volume:

(mL)

Purge Volume: 25.0

Dilution Factor: 1.0

Soil Aliquot Volume:

\begin{tabular}{|c|c|c|c|}
\hline $\begin{array}{r}\text { CAS NO. } \\
========== \\
79-01-6 \\
108-87-2 \\
78-87-5 \\
75-27-4 \\
10061-01-5 \\
108-10-1 \\
108-88-3 \\
10061-02-6 \\
79-00-5 \\
127-18-4 \\
591-78-6 \\
124-48-1 \\
106-93-4 \\
108-90-7 \\
100-41-4 \\
95-47-6 \\
179601-23-1 \\
100-42-5 \\
75-25-2 \\
98-82-8 \\
79-34-5 \\
541-73-1 \\
106-46-7 \\
95-50-1 \\
96-12-8 \\
120-82-1 \\
87-61-6\end{array}$ & 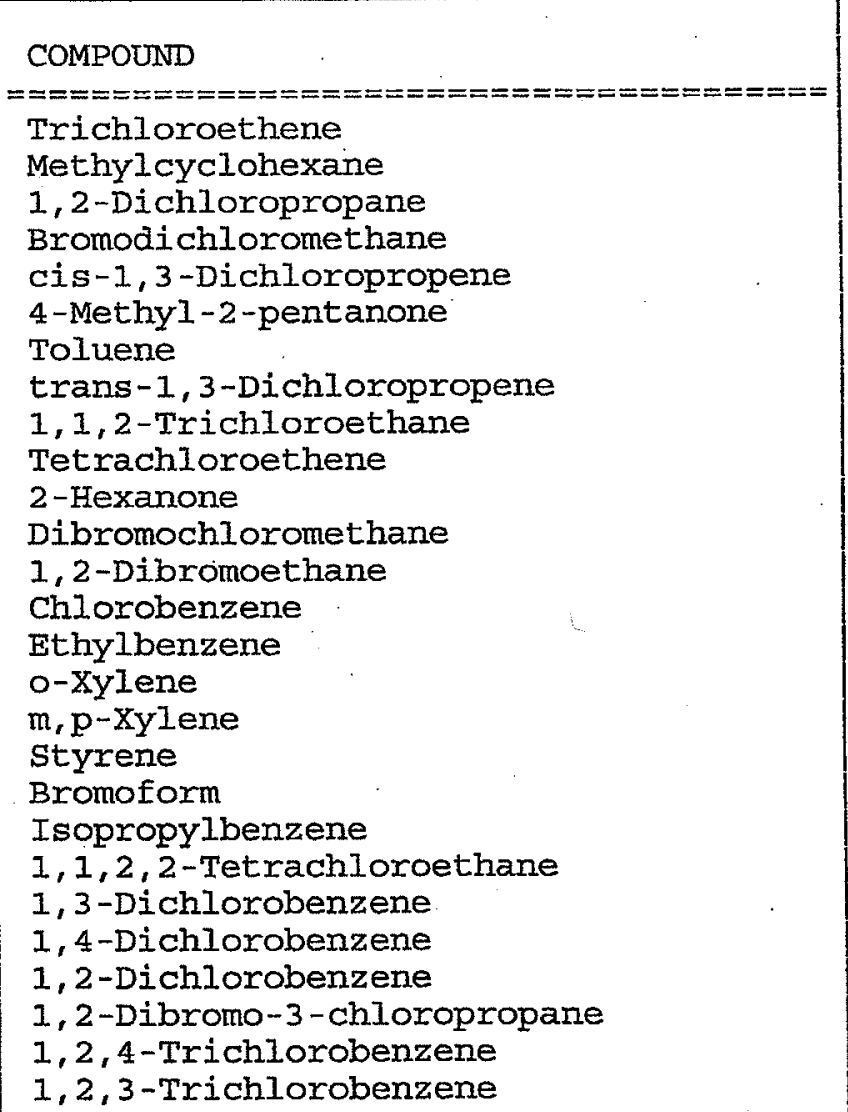 & \begin{tabular}{|cc} 
CONCENTRATION UNITS: \\
(ug $/ \mathrm{L}$ or ug/kg) & ug $/ \mathrm{L}$ \\
$=================$ \\
0.50 \\
0.50 \\
0.50 \\
0.50 \\
0.50 \\
5.0 \\
0.50 \\
0.50 \\
0.50 \\
0.50 \\
5.0 \\
0.50 \\
0.50 \\
0.50 \\
0.50 \\
0.50 \\
0.50 \\
0.50 \\
0.50 \\
0.50 \\
0.50 \\
0.50 \\
0.50 \\
0.50 \\
0.50 \\
0.50 \\
0.50
\end{tabular} & \begin{tabular}{|l|} 
Q \\
$=======$ \\
$U$ \\
$U$ \\
$U$ \\
$U$ \\
$U$ \\
$U$ \\
$U$ \\
$U$ \\
$U$ \\
$U$ \\
$U$ \\
$U$ \\
$U$ \\
$U$ \\
$U$ \\
$U$ \\
$U$ \\
$U$ \\
$U$ \\
$U$ \\
$U$ \\
$U$ \\
$U$ \\
$U$ \\
$U$ \\
$U$ \\
$U$
\end{tabular} \\
\hline
\end{tabular}


$I A-F O R M I V O A-1$

EPA SAMPLE NO.

VOLATILE ORGANICS ANALYSIS DATA SHEET

Lab Name: TESTAMERICA BURLINGTON

Contract $: 8 \mathrm{E}-00302$

Lab Code: STLV Case No.: MORRILL Mod. Ref No.:

SDG NO. : 128326

Matrix: (SOIL/SED/WATER) Water

Sample wt/vol: $25.0 \quad(\mathrm{~g} / \mathrm{mL}) \mathrm{mL}$

Level: (TRACE/LOW/MED) TRACE

$\%$ Moisture: not dec.

GC Column: DB-624

$I D: 0.53$

Soil Extract Volume:

(mm)

(uL)

Lab Sample ID: 772520

Lab File ID: 772520

Date Received: 10/22/2008

Date Analyzed: 10/23/2008

Purge Volume: 25.0
$(\mathrm{mL})$

\begin{tabular}{|c|c|c|c|}
\hline $\begin{array}{l}\text { CAS NO. } \\
\text { N========== } \\
75-71-8 \\
74-87-3 \\
75-01-4 \\
74-83-9 \\
75-00-3 \\
75-69-4 \\
75-35-4 \\
76-13-1 \\
67-64-1 \\
75-15-0 \\
79-20-9 \\
75-09-2 \\
156-60-5 \\
1634-04-4 \\
75-34-3 \\
156-59-2 \\
78-93-3 \\
74-97-5 \\
67-66-3 \\
71-55-6 \\
110-82-7 \\
56-23-5 \\
71-43-2 \\
107-06-2\end{array}$ & $\begin{array}{l}\text { Compound } \\
============================ \\
\text { Dichlorodifluoromethane } \\
\text { Chloromethane } \\
\text { Vinyl chloride } \\
\text { Bromomethane } \\
\text { Chloroethane } \\
\text { Trichlorofluoromethane } \\
1,1-D i c h l o r o e t h e n e \\
1,1,2-\text { Trichloro-1,2,2-trifluoroethane } \\
\text { Acetone } \\
\text { Carbon disulfide } \\
\text { Methyl acetate } \\
\text { Methylene chloride } \\
\text { trans-1,2-Dichloroethene } \\
\text { Methyl tert-butyl ether } \\
1,1-D i c h l o r o e t h a n e \\
\text { cis-1, } 2 \text {-Dichloroethene } \\
2-\text { Butanone } \\
\text { Bromochloromethane } \\
\text { Chloroform } \\
1,1,1-\text { Trichloroethane } \\
\text { Cyclohexane } \\
\text { Carbon tetrachloride } \\
\text { Benzene } \\
1,2-D i c h l o r o e t h a n e\end{array}$ & 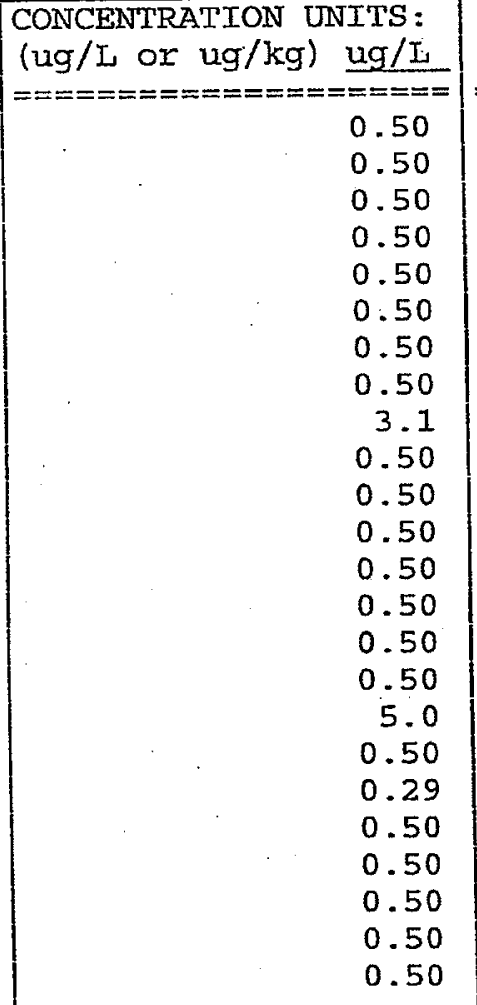 & $\begin{array}{l}\text { Q }======== \\
U \\
U \\
U \\
U \\
U \\
U \\
U \\
U \\
J \\
U \\
U \\
U \\
U \\
U \\
U \\
U \\
U \\
U \\
J \\
U \\
U \\
U \\
U \\
U\end{array}$ \\
\hline
\end{tabular}

Report 1,4-Dioxane for Low-Medium VOA analysis only 
Lab Name: TESTAMERICA BURLINGTON

Lab Code: STLV Case No.: MORRILI

Matrix: (SOIL/SED/WATER) Water

Sample wt/vol: 25.0

$\langle\mathrm{g} / \mathrm{mL}\rangle \mathrm{mL}$

Leve1: (TRACE/LOW/MED) TRACE

- Moisture: not dec.

GC Column: $\mathrm{DB}-624$

ID $: 0.53$

$(\mathrm{mm})$

(uL)

Contract: $8 E-00302$

Mod. Ref No.:
SDG No. : 128326
Soil Extract Volume:

Purge Volume: 25.0
Lab Sample ID: 772520

Lab File ID: 772520

Date Received: 10/22/2008

Date Analyzed: 10/23/2008

Dilution Factor: 1.0

Soil Aliquot Volume:

(uts)

(mL)

\begin{tabular}{|c|c|c|c|}
\hline $\begin{array}{r}\text { CAS NO. } \\
=========== \\
79-01-6 \\
108-87-2 \\
78-87-5 \\
75-27-4 \\
10061-01-5 \\
108-10-1 \\
108-88-3 \\
10061-02-6 \\
79-00-5 \\
127-18-4 \\
591-78-6 \\
124-48-1 \\
106-93-4 \\
108-90-7 \\
100-41-4 \\
95-47-6 \\
179601-23-1 \\
100-42-5 \\
75-25-2 \\
98-82-8 \\
79-34-5 \\
541-73-1 \\
106-46-7 \\
95-50-1 \\
96-12-8 \\
120-82-1 \\
87-61-6\end{array}$ & 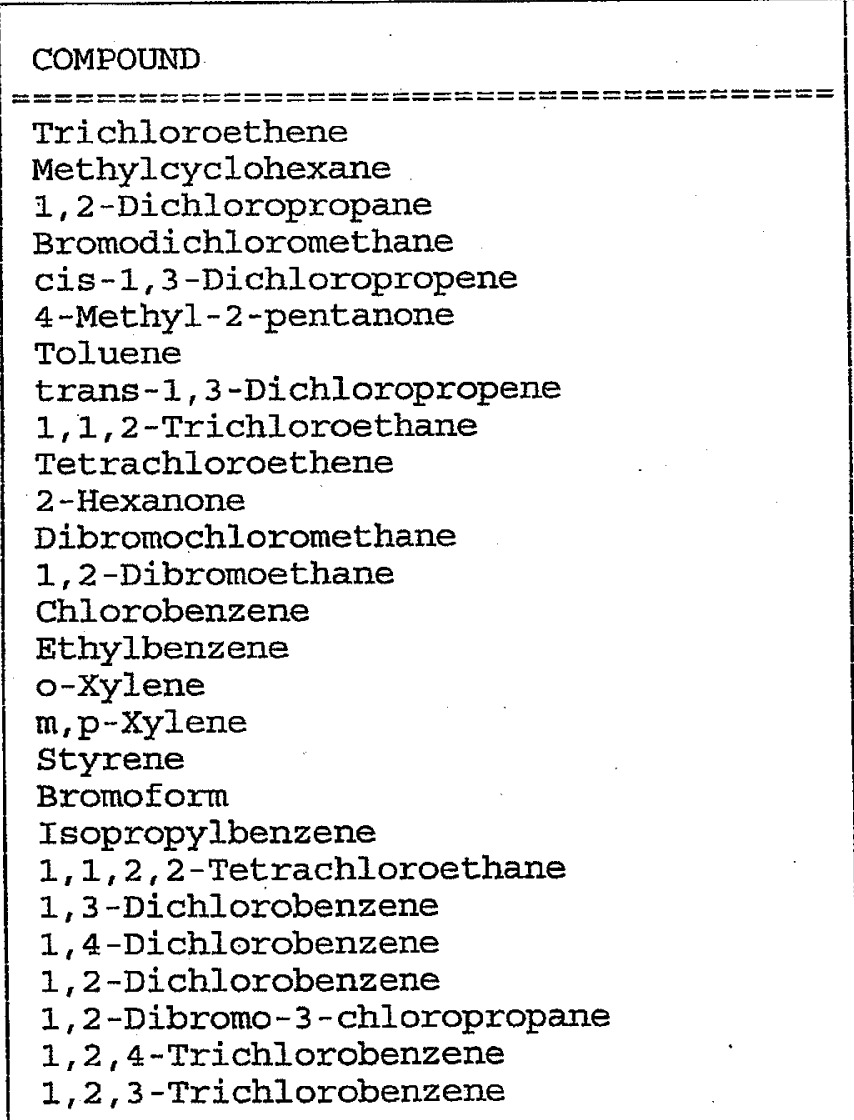 & $\begin{array}{|cc|}\text { CONCENTRATION UNITS: } \\
\text { (ug } / \mathrm{L} \text { or ug } / \mathrm{kg}) & \underline{u g} / \mathrm{L} \\
============== \pm===== \\
0.50 \\
0.50 \\
0.50 \\
0.24 \\
0.50 \\
5.0 \\
0.50 \\
0.50 \\
0.50 \\
0.50 \\
5.0 \\
0.50 \\
0.50 \\
0.50 \\
0.50 \\
0.50 \\
0.50 \\
0.50 \\
0.50 \\
0.50 \\
0.50 \\
0.50 \\
0.50 \\
0.50 \\
0.50 \\
0.50 \\
0.50\end{array}$ & $\begin{array}{l}\text { Q }======= \\
U \\
U \\
U \\
J \\
U \\
U \\
U \\
U \\
U \\
U \\
U \\
U \\
U \\
U \\
U \\
U \\
U \\
U \\
U \\
U \\
U \\
U \\
U \\
U \\
U \\
U \\
U\end{array}$ \\
\hline
\end{tabular}


Lab Name: TESTAMERICA BURLINGTON

Iab Code: STIV

Case NO.: MORRILL

Mod. Ref No.:
Contract: 8E-00302

SDG NO.: 128326
Matrix: (SOIL/SED/WATER) Water

Sample wt/vol: 25.0

$(\mathrm{g} / \mathrm{mL}) \mathrm{mL}$

Leve1: (TRACE/LOW/MED) TRACE

$\because$ Moisture: not dec.

GC Column: DB-624

ID $=0.53$

Soil Extract Volume:

Purge Volume: 25.0
Lab Sample ID: 772521

Lab File ID: 772521

Date Received:

Date Analyzed: 10/24/2008

(mm) Dilution Factor: 1.0

(ur) Soil Aliquot Volume:

(uL)

\begin{tabular}{|c|c|c|c|}
\hline $\begin{array}{c}\text { CAS NO. } \\
========== \\
75-71-8 \\
74-87-3 \\
75-01-4 \\
74-83-9 \\
75-00-3 \\
75-69-4 \\
75-35-4 \\
76-13-1 \\
67-64-1 \\
75-15-0 \\
79-20-9 \\
75-09-2 \\
156-60-5 \\
1634-04-4 \\
75-34-3 \\
156-59-2 \\
78-93-3 \\
74-97-5 \\
67-66-3 \\
71-55-6 \\
110-82-7 \\
56-23-5 \\
71-43-2 \\
107-06-2\end{array}$ & $\begin{array}{l}\text { CoMPOUND } \\
============================\mid \\
\text { Dichlorodifluoromethane } \\
\text { Chloromethane } \\
\text { Vinyl chloride } \\
\text { Bromomethane. } \\
\text { Chloroethane } \\
\text { Trichlorofluoromethane } \\
1,1-D i c h l o r o e t h e n e \\
1,1,2-\text { Trichloro-1,2,2-trifluoroethane } \\
\text { Acetone } \\
\text { Carbon disulfide } \\
\text { Methyl acetate } \\
\text { Methylene chloride } \\
\text { trans-1,2-Dichloroethene } \\
\text { Methyl tert-butyl ether } \\
1,1-D i c h l o r o e t h a n e \\
\text { cis-1,2-Dichloroethene } \\
2-\text { Butanone } \\
\text { Bromochloromethane } \\
\text { Chloroform } \\
1,1,1-\text { Trichloroethane } \\
\text { Cyclohexane } \\
\text { Carbon tetrachloride } \\
\text { Benzene } \\
1,2-D i c h l o r o e t h a n e\end{array}$ & 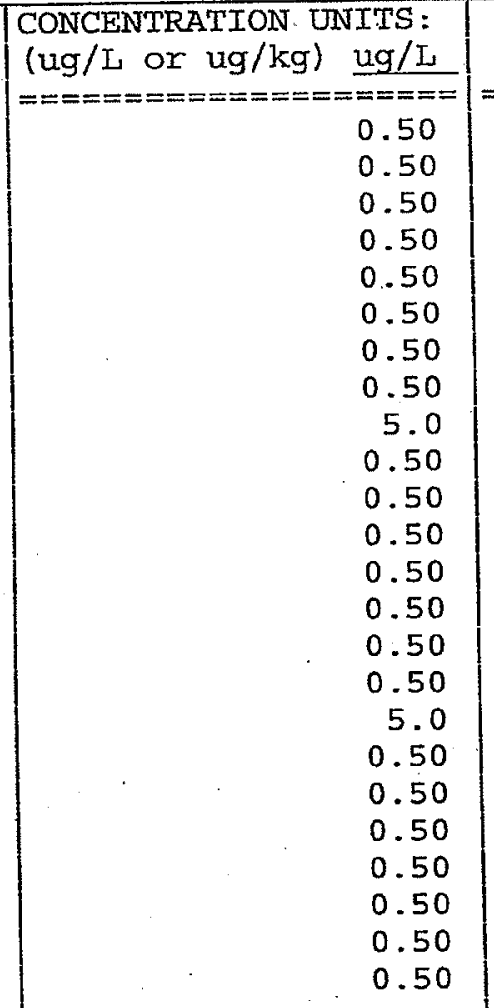 & $\begin{array}{l}======= \\
U \\
U \\
U \\
U \\
U \\
U \\
U \\
U \\
U \\
U \\
U \\
U \\
U \\
U \\
U \\
U \\
U \\
U \\
U \\
U \\
U \\
U \\
U \\
U\end{array}$ \\
\hline
\end{tabular}

Report 1,4-Dioxane for Low-Medium VOA analysis only 
Lab Name: TESTAMERICA BURLINGTON

Lab Code: STLV

Case NO.: MORRILI

Contract: $8 \mathrm{E}-00302$

Matrix: (SOII/SED/WATER) Water

Sample wt/vol: 25.0

$(\mathrm{g} / \mathrm{mL}) \mathrm{mL}$

Leve1: (TRACE/LOW/MED) TRACE

$\%$ Moisture: not dec.

GC Column: DB-624

ID : 0.53

Soil Extract Volume:

Purge Volume: 25.0 ef No.:
Lab Sample ID: 772521

Lab File ID: 772521

Date Received:

Date Analyzed: 10/24/2008

Dilution Factor: 1.0

Soil Aliquot Volume:

(uL)

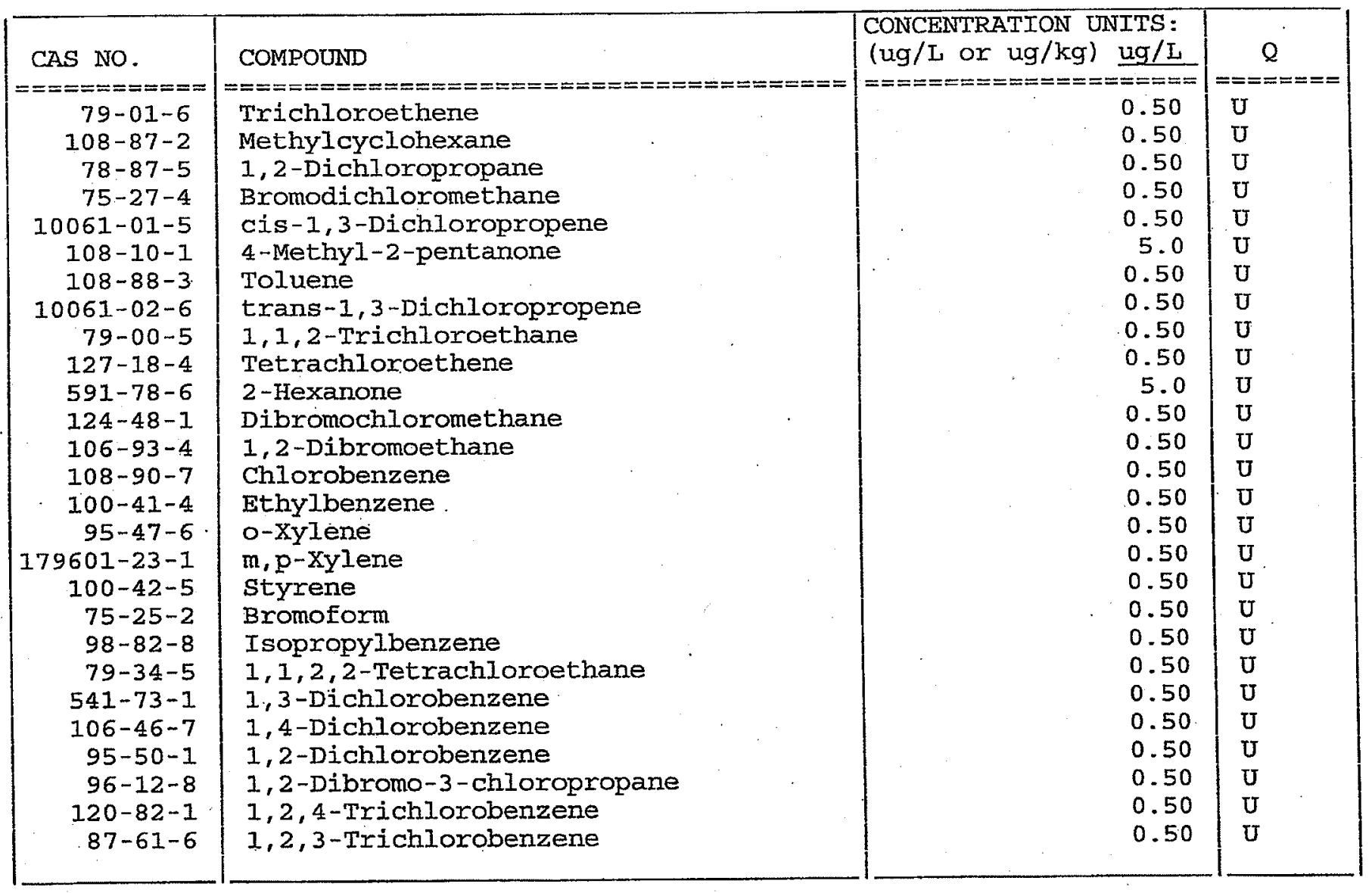

SOM01. 2 
Argonne

Environmental Science Division

Argonne National Laboratory

9700 South Cass Avenue, Bldg. 203

Argonne, IL 60439-4843

www.anl.gov 